\title{
Statistical Properties of the Signal-to-Noise Crossover Dose Based on the Hill Model as a Point of Departure for Health Risk Assessment
}

\author{
by \\ Ahalya Sivathayalan \\ M.Sc Mathematics (Statistics), Carleton University, Ottawa, ON \\ B. Sc. Eng. (Hons), University of Peradeniya, Sri Lanka.
}

A thesis submitted to the faculty of Graduate and Postdoctoral Affairs in partial fulfillment of the requirements for the degree of

\author{
Doctor of Philosophy \\ in \\ Mathematics (Statistics)
}

\begin{abstract}
School of Mathematics and Statistics
Ottawa-Carleton Institute for Mathematics and Statistics

Carleton University

Ottawa, Ontario

October 5, 2015
\end{abstract}

(C)Ahalya Sivathayalan, 2015 


\begin{abstract}
No-observed-adverse-effect-level (NOAEL) and benchmark dose (BMD) are widely accepted points of departure for human exposure guidelines. BMD has considerable merits to the NOAEL. However, BMD estimates could be model dependent if the estimation requires extrapolation beyond the range of experimental data. Signal-tonoise crossover dose (SNCD) is recently introduced to overcome this limitation, where the focus was point estimation of the SNCD using NTP data sets. In this research, we introduce three new approaches to estimate the SNCD. Further to illustrate the characteristics of the BMD to SNCD, a new analytical approach to estimate the BMD is introduced and comparison of these PoDs is carried out using the NTP data sets.

The first part involves estimation and assessment of Hill model parameters and an introduction of an analytical approach to estimate the benchmark dose. Accuracy and precision of these estimates are investigated for all possible combination of four shapes of the Hill curves, two dose levels and four number of trials per dose and effects of these factors on these estimates are reported.

The second part includes estimation and assessment of SNCD using three different methods. Results show that the analytical approach with bias correction is the best. To estimate the realized bias, multiple regression equation as a function of Hill model parameters, number of dose levels, number of trials per dose and maximum dose; and additional five new approaches to estimate the bias are introduced and compared. Our analysis show that the best approach to estimate the bias is the multiple regression approach. It is used in the application of the above illustrated
\end{abstract}


theories.

The developed theories are applied in the analysis of NTP datasets and the corresponding PoDs are estimated. Comparison of BMD to SNCD, BMDL to SNCDL and computation of extra risks at SNCD and SNCDL are carried out to illustrate the advantages of one PoD over the other. Results show that SNCD0.67 is comparable to BMD10 and SNCDL0.67 to BMDL10 and confirming the conclusion that there is a need to choose smaller p $(<0.67)$ of SNCD which could be comparable to BMD05 or BMD01 


\section{DEDICATION}

This research in new point of departure for human exposure guidelines is dedicated to my little brother, Peethamparam Ravialahan, who was a non-smoker himself; and born and grew up in a family without the history of cancer, but lost his precious life to lung cancer. He migrated to a foreign country, worked hard to build a life on his

own. Goals and aspirations that he had for himself and his family had been unfairly taken away by his untimely passing due to this illness. He put others' welfare ahead him even when he was suffering. He is our hero and had been an inspiration to all of us.

We all miss him terribly!

I hope that my research in environmental toxicity will contribute to solving this problem that this world is facing currently. 


\section{ACKNOWLEDGEMENTS}

I would like to express my sincere gratitude and appreciation to Dr. Patrick Farrell and Dr. Daniel Krewski for all the help and guidance they have provided me

over the years. A great collaboration was felt at every meeting over the past four years. This has helped me to become a better statistician and researcher.

I would also like to thank Dr. Salomon Sand for providing the NTP datasets that is used in this thesis.

I would also like to thank the members of my academic committee, Dr. Robert Platt of McGill University, Dr. Paul Villeneuve of Carleton University, Dr. Jason Nielsen of Carleton University and Dr. Julian Little of University of Ottawa for taking the time to read and to provide their invaluable comments on my thesis research.

This doctoral research was supported by Ontario Graduate Scholarship (OGS) and scholarships from Faculty of Graduate Studies and Research; and School Mathematics and Statistics, Carleton University.

I would like to thank Dr. Shirley Mills for encouraging me to pursue my doctoral studies. She has been a positive influence professionally in the early stages of my career. I would also like to thank all the faculty with whom I have taken courses over these years.

I would like to thank Barbara Nicholl, Cate Palmer, Ken Small, Kevin Crosby, Lana Graham, Nicole Begnoche and Tracie Barkley for their prompt help over the years and making my life as a student in our department enjoyable. Further, I would like to thank Ryan Taylor for helping me with remote computing promptly whenever needed and John Stewart for all the other computer help. This gave me more time 
with my family.

Lastly and more importantly, I would like to thank my family for their love and continued support. I would like to thank my husband, Dr. Siva Sivathayalan, and our children, Janani and Ashok, for their patience and love over the years even when I spent most of my time working on my thesis. I would also like to thank my parents, Vaithilingam Peethamparam and Sakunthala Peethamparam, for looking after my children whenever needed and all the sacrifices they made over the years, and my brothers for their encouragement support.

The last five months leading to my defense have been the toughest time of my personal life. I would like to thank my brother Dr. Peethamparam Anpalahan for talking to me each and every day to listen and help us get through the ordeal of losing our little brother, Ravialahan. I would like to further thank my husband, our children, other family members and friends for providing me all the support to deal with this pain. They all gave me the strength to complete this work. 


\section{CONTENTS}

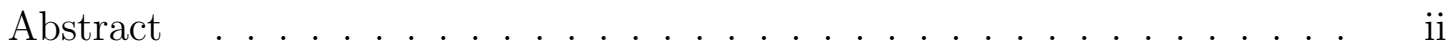

Dedication $\ldots \ldots \ldots \ldots \ldots \ldots \ldots \ldots \ldots \ldots \ldots \ldots \ldots \ldots \ldots \ldots$

Acknowledgments . . . . . . . . . . . . . . . . v v

Contents .................................. vii

List of Tables . . . . . . . . . . . . . . . . . xi

List of Figures . . . . . . . . . . . . . . . . xiv

1 Introduction 1

1.1 Motivation and Statement of Problem . . . . . . . . . . . . 1

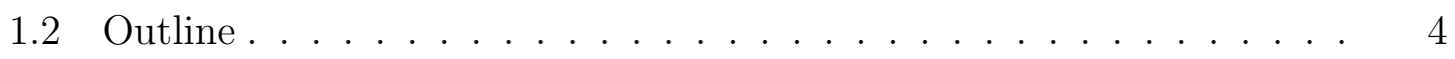

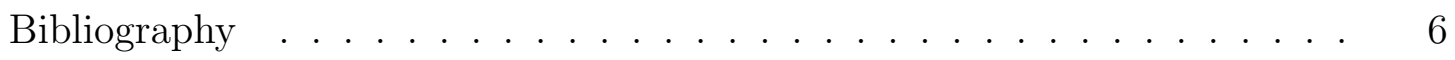

2 Statistical Properties of Hill Model Parameter and Benchmark Dose Estimates 8

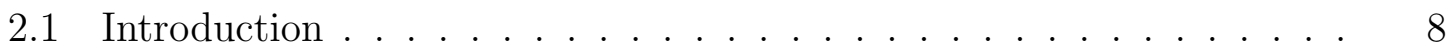

2.2 Hill Model Characterization of the Dose-Response Relationship . . . . 11

2.2 .1 Model Specification . . . . . . . . . . . . . . . . . . . . 11

2.2.2 Dose-Response Characteristics of the selected Hill Model . . . 12

2.3 Point of Departure: Benchmark Dose . . . . . . . . . . . . 16

2.4 Estimation of Hill Model Parameters and Benchmark Dose . . . . . . 17

2.4.1 Hill Model Parameter Estimates . . . . . . . . . . . . . . . . 17

2.4.2 Estimation of Benchmark Dose . . . . . . . . . . . . 19

2.5 Simulation Study . . . . . . . . . . . . . . . . . . . . . . 21

2.6 Results and Discussion . . . . . . . . . . . . . . . 23

2.7 Summary and Conclusion $\ldots \ldots \ldots \ldots \ldots$




3 Estimation and Assessment of Signal-to-Noise Crossover Dose 39

3.1 Introduction . . . . . . . . . . . . . . . . . . 39

3.2 Signal-to-Noise Crossover Dose . . . . . . . . . . . . . . . . 44

3.3 Estimation of Signal-to-Noise Crossover Dose . . . . . . . . . . . . 45

3.3.1 Analytical Method ... . . . . . . . . . . . 45

3.3.2 Bootstrap Method ................ 51

3.4 Simulation Study . . . . . . . . . . . . . . . . 51

3.4.1 Analytical Method ... . . . . . . . . . . . 52

3.4.2 Bootstrap Method ................ 53

3.4.2.1 Parametric Bootstrap Method . . . . . . . . . 53

3.4.2.2 Non-parametric Bootstrap Method . . . . . . . . 55

3.5 Results and Discussion . . . . . . . . . . . . . . . . 55

3.5.1 Estimation of Signal-to-Noise Crossover Dose . . . . . . . . 55

3.5.1.1 Analytical Method ............ 56

3.5.1.2 Parametric Bootstrap Method . . . . . . . . . 62

3.5.1.3 Non-parametric Bootstrap Method . . . . . . . . 67

3.5.2 Multiple Regression . . . . . . . . . . . . . . . . . . 70

3.5.2.1 Qualitative Explanatory Variables ........ 70

3.5.2.2 Quantitative Explanatory Variables . . . . . . . . 84

3.5.3 Estimation and Assessment of Bias Correction . . . . . . . . . 86

3.6 Summary and Conclusion . . . . . . . . . . . . . . 88

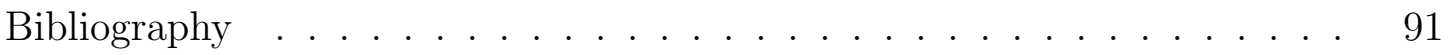

4 Empirical Comparison of the Signal-to-noise Crossover Dose and the Bench$\begin{array}{ll}\text { mark Dose } & 95\end{array}$

4.1 Introduction . . . . . . . . . . . . . . . . . . 95

4.2 Data . . . . . . . . . . . . . . . . . . . . 101

4.2.1 Model Fitting . . . . . . . . . . . . . . . . . 101 
4.3 Equations and Methods . . . . . . . . . . . . . . . . 102

4.3.1 Estimates of BMD, BMDL and Ratios _. . . . . . . . . 102

4.3.2 Estimates of SNCD, SNCDL and Ratios . . . . . . . . . 103

4.3.3 Ratios of Different PoDs . . . . . . . . . . . . . . . . 105

4.3.4 Extra Risk at SNCDL, SNCD and SNCDU . . . . . . . . 106

4.4 Results and Discussion . . . . . . . . . . . . . . . 107

4.4 .1 Bench Mark Dose Ratios . . . . . . . . . . . . . . . . 107

4.4.2 Signal-to-noise Crossover Dose Ratios . . . . . . . . . . . . 109

4.4.2.1 Uncorrected . . . . . . . . . . . . . . . . . . 109

4.4.2.2 Bias-corrected . . . . . . . . . . . . . . . . 110

4.4 .3 Ratios of BMD to SNCD . . . . . . . . . . . . . . . . 111

4.4.3.1 Simulation Results of Ratio of BMD to SNCD . . . . 111

4.4.3.2 Results for Ratio of BMD to SNCD from Real Datasets114

4.4.4 Ratios of BMDL and SNCDL . . . . . . . . . . . . 119

4.4.4.1 Ratio of BMDL to Uncorrected SNCDL . . . . . 120

4.4.4.2 Ratio of BMDL to Bias-corrected SNCDL . . . . . 122

4.4.5 Extra Risk at SNCDL and SNCD . . . . . . . . . . . . 124

4.4.5.1 Uncorrected . . . . . . . . . . . . . . . . . . 124

4.4.5.2 Bias-corrected . . . . . . . . . . . . . 126

4.5 Summary and Conclusion . . . . . . . . . . . . . . . 128

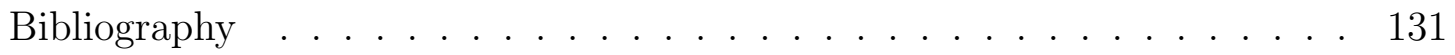

5 Summary, Conclusion and Future Work 134

5.1 Summary and Conclusion . . . . . . . . . . . . . . 134

5.2 Future Work . . . . . . . . . . . . . . . . . . . . 136 


\section{APPENDIX}

A Appendix of Chapter 2

A.1 Distribution of Maximum Likelihood Estimates of Hill Model Param-



A.1.1 Distribution of Hill Model Parameter Estimates . . . . . . . . 138

A.1.2 Distribution of BMD Estimates . . . . . . . . . . . . . 140

A.2 Characteristics of Hill Model Curves for larger Background Incident • 142

A.3 Tabulation Hill Model Parameter and BMD Estimates . . . . . . . . 148

A.3.1 Properties of Hill Model Parameter Estimates . . . . . . . . . 148

A.3.2 Properties of Hill Model Parameter Variance Estimates . . . . 151

A.3.3 Properties of Hill Model Parameter Covariance Estimates . . . 154

A.3.4 Properties of Benchmark Dose Estimates based on Extra Risk 157

A.3.5 Properties of Benchmark Dose Estimates based on Additional Risk . . . . . . . . . . . . . . . 160

B Appendix of Chapter $3 \quad 163$

B.1 Analytical Method Results . . . . . . . . . . . . . . . . 163

B.1.1 Preliminary Investigation of Signal-to-Noise Response . . . . . 163

B.1.2 Results of SNCD Estimates . . . . . . . . . . . 166

B.2 Bootstrap Method . . . . . . . . . . . . . . . 178

B.2.1 Parametric Bootstrap Method Results . . . . . . . . . . . . 178

B.2.2 Non-parametric Bootstrap Method Results . . . . . . . . . . 185

$\begin{array}{ll}\text { C Appendix of Chapter } 4 & 194\end{array}$

C.1 Histogram of Ratio of BMD and SNCD based on Simulation Results . 194 


\section{TABLES}

\section{Table}

3.1 Multiple Regression Parameter Estimates (Analytical) when $p=0.67 \quad 73$

3.2 Multiple Regression Parameter Estimates (Analytical) when $p=1.0$. 74

3.3 Analytical Approach P-values for $p=0.67 \ldots$. . . . . . . . . 76

3.4 Analytical Approach P-values for $p=1.0 \ldots \ldots$. . . . . . . . 76

3.5 Multiple Regression Parameter Estimates (Parametric Bootstrap) when

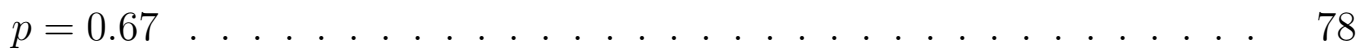

3.6 Multiple Regression Parameter Estimates (Parametric Bootstrap) when $p=1.0 \ldots \ldots \ldots \ldots \ldots$

3.7 Parametric Boostrap Approach P-values for $p=0.67$. . . . . . . . 80

3.8 Parametric Boostrap Approach P-values for $p=1.0$. . . . . . . . . 80

3.9 Multiple Regression Parameter Estimates (Non-parametric Bootstrap) when $p=0.67 \ldots \ldots \ldots$. . . . . . . . . . . . . 81

3.10 Multiple Regression Parameter Estimates (Non-parametric Bootstrap) when $p=1.0 \ldots \ldots \ldots$. . . . . . . . . . . . . . . 82

3.11 Non-parametric Boostrap Approach P-values for $p=0.67 \ldots$. . . . 83

3.12 Non-parametric Boostrap Approach P-values for $p=1.0$. . . . . . 83

3.13 Reduced Model for Biases of SNCD1.0 and SNCD0.67 with Quantitative Variables . . . . . . . . . . . . . . . . . . 86

4.1 Counts of BMD, BMDL and the Corresponding Ratios . . . . . . . 103

4.2 Counts of SNCD, SNCDL and the Corresponding Ratios . . . . . . . 104

4.3 Counts of Bias-corrected SNCD, SNCDL and the Corresponding Ratios 105

4.4 Quantiles of Ratios of BMDL to BMD . . . . . . . . . . . . 107

4.5 Quantiles for Ratios of SNCDL to SNCD . . . . . . . . . . . . 109 
4.6 Quantiles for Ratios of Bias-corrected SNCDL to SNCD . . . . . . . 110

4.7 Quantiles for Ratios of BMD to SNCD . . . . . . . . . . . . 115

4.8 Quantiles for Ratios of BMD to Bias-corrected SNCD . . . . . . . . . 117

4.9 Quantiles for Ratios of BMDL to SNCDL . . . . . . . . . . . . . 120

4.10 Quantiles for Ratios of BMDL to Bias-corrected SNCDL . . . . . . . 122

4.11 Quantiles for Risk of Uncorrected Signal-to-Noise Crossover Dose . 124

4.12 Quantiles for Risk of Bias-corrected Signal-to-Noise Crossover Dose 126

A.1 Properties of $\alpha$ when $(\alpha, \kappa, \eta)=(0.1,4,3) \ldots \ldots \ldots \ldots$

A.2 Properties of MLEs for $\kappa \ldots \ldots \ldots \ldots$. . . . . . . . . 148

A.3 Properties of MLEs for $\eta \ldots \ldots \ldots$. . . . . . . . . . . 149

A.4 Properties of MLEs for $\alpha \ldots \ldots \ldots$. . . . . . . . . 150

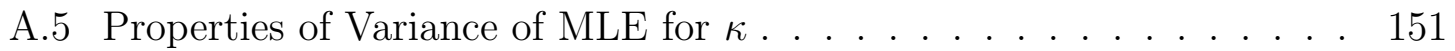

A.6 Properties of Variance of MLE for $\eta$. . . . . . . . . . . . . . . 152

A.7 Properties of Variance of MLE for $\alpha \ldots \ldots$. . . . . . . . . . 153

A.8 Properties of Covariance of MLE for $\kappa$ and $\eta$. . . . . . . . . . . 154

A.9 Properties of Covariance of MLE for $\kappa$ and $\alpha \ldots \ldots . . . . . . .155$

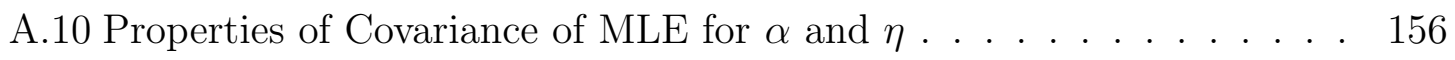

A.11 Properties of Estimate of BMD01 based on Extra Risk . . . . . . . . 157

A.12 Properties of Estimate of BMD05 based on Extra Risk . . . . . . . . 158

A.13 Properties of Estimate of BMD10 based on Extra Risk . . . . . . . . 159

A.14 Properties of Estimate of BMD01 based on Additional Risk . . . . . 160

A.15 Properties of Estimate of BMD05 based on Additional Risk . . . . . 161

A.16 Properties of Estimate of BMD10 based on Additional Risk . . . . . 162

B.1 Comparison of Expectations at Dose Points . . . . . . . . . . . 164

B.2 Comparison of Variances at Dose Points . . . . . . . . . . . . 165

B.3 Results of Coverage Probability of True SNR . . . . . . . . . . . . 165

B.4 SNCDs on Hill Model when $\alpha=0.01, \kappa=4, \eta=3$ and $k=4 \ldots . . .166$ 
B.5 SNCDs on Hill Model when $\alpha=0.01, \kappa=4, \eta=3$ and $k=6$. . . . . 167

B.6 SNCDs on Hill Model when $\alpha=0.01, \kappa=4, \eta=3$ and $k=8 \ldots$. . . 168

B.7 SNCDs on Hill Model when $\alpha=0.01, \kappa=5, \eta=1$ and $k=4 \ldots$. . . 169

B.8 SNCDs on Hill Model when $\alpha=0.01, \kappa=5, \eta=1$ and $k=6$. . . . . 170

B.9 SNCDs on Hill Model when $\alpha=0.01, \kappa=5, \eta=1$ and $k=8$. . . . . 171

B.10 SNCDs on Hill Model when $\alpha=0.01, \kappa=7, \eta=2$ and $k=4$. . . . . 172

B.11 SNCDs on Hill Model when $\alpha=0.01, \kappa=7, \eta=2$ and $k=6$. . . . . 173

B.12 SNCDs on Hill Model when $\alpha=0.01, \kappa=7, \eta=2$ and $k=8$. . . . . 174

B.13 SNCDs on Hill Model when $\alpha=0.01, \kappa=8, \eta=5$ and $k=4 \ldots . . . \quad 175$

B.14 SNCDs on Hill Model when $\alpha=0.01, \kappa=8, \eta=5$ and $k=6 \ldots$. . . 176

B.15 SNCDs on Hill Model when $\alpha=0.01, \kappa=8, \eta=5$ and $k=8 \ldots \ldots$. . 177

B.16 SNCDs on Hill Model when $\alpha=0.01, \kappa=4, \eta=3$ and $k=4 \ldots$. . . 178

B.17 SNCDs on Hill Model when $\alpha=0.01, \kappa=4, \eta=3$ and $k=6 \ldots$. . . 179

B.18 SNCDs on Hill Model when $\alpha=0.01, \kappa=5, \eta=1$ and $k=4 \ldots . . . \quad 180$

B.19 SNCDs on Hill Model when $\alpha=0.01, \kappa=5, \eta=1$ and $k=6$. . . . . . 181

B.20 SNCDs on Hill Model when $\alpha=0.01, \kappa=7, \eta=2$ and $k=4 \ldots . . . \quad 182$

B.21 SNCDs on Hill Model when $\alpha=0.01, \kappa=7, \eta=2$ and $k=6 \ldots$. . . 183

B.22 SNCDs on Hill Model when $\alpha=0.01, \kappa=8, \eta=5$ and $k=4 \ldots \ldots$. 184

B.23 SNCDs on Hill Model when $\alpha=0.01, \kappa=8, \eta=5$ and $k=6 \ldots . . . \quad 185$

B.24 SNCDs on Hill Model when $\alpha=0.01, \kappa=4, \eta=3$ and $k=4 \ldots$. . . 186

B.25 SNCDs on Hill Model when $\alpha=0.01, \kappa=4, \eta=3$ and $k=6 \ldots$. . . 187

B.26 SNCDs on Hill Model when $\alpha=0.01, \kappa=5, \eta=1$ and $k=4 \ldots . . . \quad 188$

B.27 SNCDs on Hill Model when $\alpha=0.01, \kappa=5, \eta=1$ and $k=6$. . . . . 189

B.28 SNCDs on Hill Model when $\alpha=0.01, \kappa=7, \eta=2$ and $k=4 \ldots . . . \quad 190$

B.29 SNCDs on Hill Model when $\alpha=0.01, \kappa=7, \eta=2$ and $k=6$. . . . . . 191

B.30 SNCDs on Hill Model when $\alpha=0.01, \kappa=8, \eta=5$ and $k=4$. . . . . . 192

B.31 SNCDs on Hill Model when $\alpha=0.01, \kappa=8, \eta=5$ and $k=6 \ldots . . . \quad 193$ 


\section{FIGURES}

\section{Figure}

2.1 Dose Response Curves for Selected Hill Model Parameters $\kappa$ and $\eta$ with

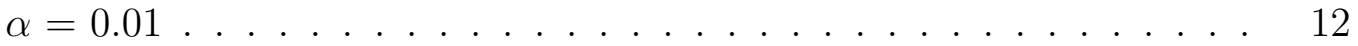

2.2 Dose Response for $\eta=(1,2,3,5)$ for $\kappa / d_{k}=(0.50-1.25) \ldots \ldots$

2.3 Dose Response for $\kappa / d_{k}=(0.500,0.625,0.875,1.00)$ for $\eta=(1,2,3$, $4,5,6,7) \ldots \ldots \ldots \ldots \ldots \ldots \ldots \ldots$

2.4 Histogram for S-shaped Hill Model Parameter Estimates when $\alpha=0.0124$

2.5 Histogram for S-shaped Hill Model Parameter Estimates when $\alpha=0.125$

2.6 Variation of Properties of Estimates of $\kappa \ldots \ldots$

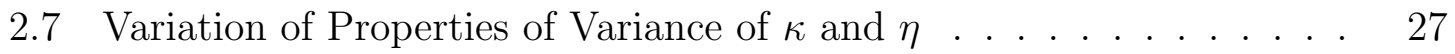

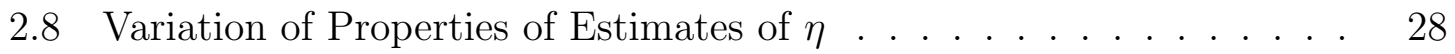

2.9 Histogram for BMD Estimates on S-shaped Hill Curves . . . . . . . . 29

2.10 Variation of Properties of Estimates of BMD10 . . . . . . . . . . 31

3.1 Graphical Display of SNCDL, SNCD and SNCDU . . . . . . . . . 49

3.2 Variation of True Bias for SNCD0.67 and SNCD1.0 using Analytical Method ......................... 56

3.3 Variation of Coverage Probability based on (SNCDL, SNCDU) for SNCD0.67 and SNCD1.0 using Analytical Method . . . . . . . . . 58

3.4 Variation of Coverage Probability based on $\left(S N C D_{05}, S N C D_{95}\right)$ for SNCD0.67 and SNCD1.0 using Analytical Method . . . . . . . . . . 60

3.5 Variation of True Bias for SNCD0.67 and SNCD1.0 using Parametric Bootstrap Method . . . . . . . . . . . . . 62

3.6 Variation of Coverage Probability based on (SNCDL, SNCDU) for SNCD0.67 and SNCD1.0 using Parametric Bootstrap Method . . . 
3.7 Variation of Coverage Probability based on $\left(S N C D_{05}, S N C D_{95}\right)$ for SNCD0.67 and SNCD1.0 using Parametric Bootstrap Method . . . .

3.8 Variation of True Bias for SNCD0.67 and SNCD1.0 using Non-parametric

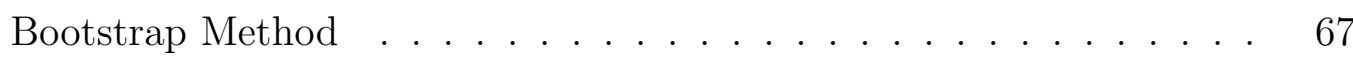

3.9 Variation of Coverage Probability based on (SNCDL, SNCDU) for SNCD0.67 and SNCD1.0 using Non-parametric Bootstrap Method . . 68

3.10 Variation of Coverage Probability based on $\left(S N C D_{05}, S N C D_{95}\right)$ for SNCD0.67 and SNCD1.0 using Non-parametric Bootstrap Method . .

3.11 Actual vs Fitted Bias of SNCD0.67 for Subset of Data . . . . . . . . 85

3.12 Actual vs Fitted Bias of SNCD1.0 for Subset of Data . . . . . . . . . 85

3.13 Coverage Probabilities for Five different Bias-corrected Estimates of SNCD0.67 and SNCD1.0 . . . . . . . . . . . . . . . 88

4.1 Histogram for Ratios of BMDL to BMD . . . . . . . . . . . . 108

4.2 Histogram for Ratios (uncorrected) of SNCDL to SNCD . . . . . . 109

4.3 Histogram for Ratios (bias-corrected) of SNCDL to SNCD . . . . . . 111

4.4 Histogram for Ratios of BMD to SNCD estimated on S-shaped Hill Model Curves . . . . . . . . . . . . . . . . . . . . . . 112

4.5 Histogram for Ratios of BMD to SNCD estimated on Convex Hill Model Curves . . . . . . . . . . . . . . . . . . . . . . . . 112

4.6 Histogram for Ratios of BMD to SNCD estimated on initial slope $>0$ Hill Model Curves . . . . . . . . . . . . . . . . . . . . . . . . . 113

4.7 Histogram for Ratios of BMD to SNCD estimated on initial slope $=0$ Hill Model Curves . . . . . . . . . . . . . . . . . . . . . . . . . 113

4.8 Histogram for Ratios of BMD10 to SNCD . . . . . . . . . . . . 115

4.9 Histogram for Ratios of BMD05 to SNCD . . . . . . . . . . . 116

4.10 Histogram for Ratios of BMD01 to SNCD . . . . . . . . . . . 116

4.11 Histogram for Ratios of BMD10 to Bias-corrected SNCD . . . . . . . 118 
4.12 Histogram for Ratios of BMD05 to Bias-corrected SNCD . . . . . . . 119

4.13 Histogram for Ratios of BMD01 to Bias-corrected SNCD . . . . . . . 119

4.14 Histogram for Ratios of BMDL10 to SNCDL . . . . . . . . . . . . . 120

4.15 Histogram for Ratios of BMDL05 to SNCDL . . . . . . . . . . . . . 121

4.16 Histogram for Ratios of BMDL01 to SNCDL . . . . . . . . . . . . 121

4.17 Histogram for Ratios of BMDL10 to Bias-corrected SNCDL . . . . . 122

4.18 Histogram for Ratios of BMDL05 to Bias-corrected SNCDL . . . . . 123

4.19 Histogram for Ratios of BMDL01 to Bias-corrected SNCDL . . . . . 123

4.20 Histogram for Extra Risk at SNCDL . . . . . . . . . . . . . 125

4.21 Histogram for Extra Risk at SNCD . . . . . . . . . . . . 125

4.22 Histogram for Extra Risk at SNCDU . . . . . . . . . . . 126

4.23 Histogram for Extra Risk at Bias-corrected SNCDL . . . . . . . . . . 127

4.24 Histogram for Extra Risk at Bias-corrected SNCD . . . . . . . . . . . 127

4.25 Histogram for Extra Risk at Bias-corrected SNCDU . . . . . . . . . . 128

A.1 Histogram for MLEs of Convex Hill Parameters when $n_{i}=50$. . . . . 138

A.2 Histogram for MLEs of Concave with initial slope $>0$ Hill Curve when $n_{i}=50 \ldots \ldots \ldots \ldots \ldots$

A.3 Histogram for MLEs of Concave with initial slope $=0$ Hill Curve when $n_{i}=50 \ldots \ldots \ldots \ldots \ldots \ldots \ldots$

A.4 Histogram for BMD Estimates on Convex Hill Curves when $n_{i}=100 \quad 140$

A.5 Histogram for BMD Estimates on Convex Hill Curves when $n_{i}=500141$

A.6 Histogram for BMD Estimates on Concave with initial slope $>0$ Hill

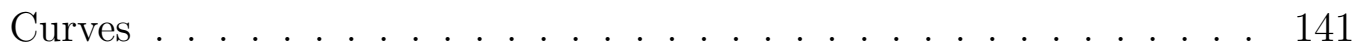

A.7 Histogram for BMD Estimates on Concave with initial slope $=0$ Hill Curves . . . . . . . . . . . . . . . . . . . . . . 142

A.8 Dose Response when $\eta=(1,2,3,5,5,7)$ with $\alpha=0.5 \ldots$. . . . . 143

A.9 Dose Response when $\kappa / d_{k}=(0.5-1.2)$ with $\alpha=0.5 \ldots$. . . . . . 144 
A.10 Histogram for S-shaped Hill Curve Parameters when $\alpha=0.5$. . . . . . 145

A.11 Histogram for Convex Hill Curve Parameters when $\alpha=0.5$. . . . . . 145

A.12 Histogram for Concave with initial slope $>0$ Hill Curve Parameters when $\alpha=0.5 \ldots \ldots \ldots$. . . . . . . . . . . . . . . . . . . . . . . . . 146

A.13 Histogram for Concave with initial slope $=0$ Hill Curve Parameters

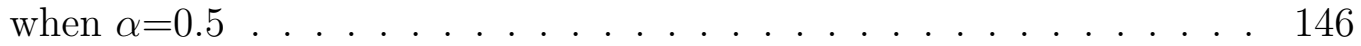

C.1 Histogram for BMD/SNCD when $\kappa=4, \eta=3, k=4, n_{i}=200 \quad \ldots$. . . 194

C.2 Histogram for BMD/SNCD when $\kappa=5, \eta=1, k=4, n_{i}=200 \quad \ldots$. . . 195

C.3 Histogram for BMD/SNCD when $\kappa=7, \eta=2, k=4, n_{i}=200 \quad \ldots$. . . 195

C.4 Histogram for BMD/SNCD when $\kappa=8, \eta=5, k=4, n_{i}=200 \ldots \ldots 6$

C.5 Histogram for BMD/SNCD when $\kappa=4, \eta=3, k=4, n_{i}=500 \ldots \ldots 6$

C.6 Histogram for BMD/SNCD when $\kappa=5, \eta=1, k=4, n_{i}=500 \quad \ldots$. . . . 197

C.7 Histogram for BMD/SNCD when $\kappa=7, \eta=2, k=4, n_{i}=500 \quad \ldots$. . . 197

C.8 Histogram for BMD/SNCD when $\kappa=8, \eta=5, k=4, n_{i}=500 \quad \ldots$. . . 198

C.9 Histogram for BMD/SNCD when $\kappa=4, \eta=3, k=6, n_{i}=50 \ldots \ldots$

C.10 Histogram for BMD/SNCD when $\kappa=5, \eta=1, k=6, n_{i}=50 \ldots \ldots 9$

C.11 Histogram for BMD/SNCD when $\kappa=7, \eta=2, k=6, n_{i}=50 \ldots \ldots 9$

C.12 Histogram for BMD/SNCD when $\kappa=8, \eta=5, k=6, n_{i}=50 \ldots \ldots$

C.13 Histogram for BMD/SNCD when $\kappa=4, \eta=3, k=6, n_{i}=200 \quad$. . . . . . 200

C.14 Histogram for BMD/SNCD when $\kappa=5, \eta=1, k=6, n_{i}=200 \quad \ldots$. . . . 201

C.15 Histogram for BMD/SNCD when $\kappa=7, \eta=2, k=6, n_{i}=200 \quad$. . . . . . 201

C.16 Histogram for BMD/SNCD when $\kappa=8, \eta=5, k=6, n_{i}=200 \quad \ldots$. . . 202

C.17 Histogram for BMD/SNCD when $\kappa=4, \eta=3, k=6, n_{i}=500 \quad \ldots$. . . . 202

C.18 Histogram for BMD/SNCD when $\kappa=5, \eta=1, k=6, n_{i}=500 \quad \ldots$. . . 203

C.19 Histogram for BMD/SNCD when $\kappa=7, \eta=2, k=6, n_{i}=500 \quad \ldots$. . . 203

C.20 Histogram for BMD/SNCD when $\kappa=8, \eta=5, k=6, n_{i}=500 \quad$. . . . . . 204 


\section{INTRODUCTION}

"All substances are poisons; there is none which is not a poison. The right dose differentiates poison from a remedy"

Paracelsus (1493-1541)

\subsection{Motivation and Statement of Problem}

Health risk assessment involves the assessment of potential adverse health effects associated with exposure to environmental, genetic, lifestyle and other factors (Krewski et. al. 2014)[8]. Qualitative risk assessment involves the identification of hazardous agents that can be harmful to human health. Quantitative risk assessment involves the development of estimates of the level of risk associated with hazardous agents, along with an indication of the level of uncertainty associated with such estimates. The overarching goal of the health risk assessment is to develop human exposure guidelines that can be expected to result in little or no risk to exposed populations.

Risk assessment as we know it today started over half a century ago. However, the concept of identifying harmful elements dates back much further. For example, there are records stating that prehistoric humans categorized some plants as harmful and others as safe. Animal venom and plant extracts were identified for hunting, warfare and assassination. There are also reports of identifying the right dose of a poison so that it can be used as an antidote. During the middle ages and early Renaissance, Paracelsus made a long lasting contribution to the as yet undeveloped science of risk assessment, stating that (1) experimentation is essential in the examination of biological responses to chemicals; (2) one should make a distinction between the therapeutic and toxic properties of chemicals; (3) these properties are sometimes but not always indistinguishable except by dose; and (4) one can ascertain a degree of specificity of chemicals and their therapeutic or toxic effects. Based on these principles, Paracelsus introduced mercury as the drug of choice for the treatment of syphilis. This has been 
historically recognized as the first clear articulation of dose-response, a concept which underlies current thinking in the field of quantitative risk assessment [2].

Industrial advances following World War II resulted in the creation of many by-products that contaminated the food chain. The use of fertilizers, pesticides and insecticides was encouraged during this period to satisfy the nutritional needs of an increasing world population. The effects of exposure to some of these chemicals were not realized until later: DDT and asbestos, for example, were widely used without realizing the consequences of exposure to these agents. The thalidomide tragedy in the 1960s underscored the importance of thorough testing before administering medications to vulnerable populations (in this case, pregnant woman) and motivated regulatory authorities and the scientific community to re-think risk assessment approaches for potentially toxic substances.

The underpinnings of modern risk science were elaborated by the US National Research Council (1983)[3], in its landmark report, Risk Assessment in the Federal Government: Managing the Process. This report laid out a four step approach to chemical risk assessment, comprised of hazard identification, dose-response assessment, exposure assessment, and risk characterization. Dose-response analysis has become a highly refined component of quantitative risk assessment, including statistical approaches to fit both empirical and biological models to toxicological and epidemiological data derived from animal and human studies, respectively (Krewski. el. al. 2002) [7].

A key aspect of modern dose-response analysis is the determination of a point on the dose-response curve that might constitute an acceptable level of human exposure to the agent of interest. One approach to establishing human exposure guidelines based on dose-response analysis is to determine a no-observed-adverse-effect level (NOAEL), originally introduced by Lehman and Fitzhugh (1954) [9], at which the risk of an adverse effect in the exposed group is not significantly different from that in 
the unexposed control group. When a NOAEL is not observed (as would be the case when risk is significantly increased at all doses considered in the study), a LOAEL (lowest-observed-adverse effect level) can be used. The NOAEL (or LOAEL, in the absence of NOAEL) is then divided by one or more uncertainty factors (UFs) to arrive at a reference dose (RfD) that may pose little or no risk to humans (Barnes and Dourson, 1988 [1]).

$$
R f D=P_{o} D / U F
$$

More generally, the risk analyst may seek an appropriate point of departure (PoD) on the dose-response curve, which serves as a starting point for the development of a human exposure guidelines. The benchmark dose (BMD) introduced by Crump (1984) [4] which represents the dose at which the risk exceeds the background risk by a specified benchmark risk level (typically 1, 5 or 10\%) called benchmark response (BMR) has received widespread attention in this regard. The benchmark dose is defined as the dose that produces a predetermined (BMR) change in the ratio of response difference for adverse effect and background to non-responding fraction.

$$
B M R=\frac{P(d)-P(0)}{1-P(0)}
$$

The BMD has a number of advantages over the NOAEL, including not being restricted to one of the experimental doses, and making use of all of the available data in its derivation (USEPA[11] and EFSA [5]); however, the EFSA argued that estimation of BMD requiring extrapolation beyond dose range would be highly model dependent. In practice, a lower confidence limit on the BMD, denoted by BMDL, is often used to take into account statistical error in the available dose-response data.

More recently, Sand et al. (2011)[10] introduced the concept of a signal-to-noise crossover dose (SNCD) as an alternative PoD for use in quantitative risk assessment. Conceptually, the SNCD can be thought of as the point at which the uncertainty in the biological dose-response signal is equal to the background variation in response 
or noise. Sand et. al. (2011) conducted comparisons of the SNCD calculated from a large number of animal cancer bioassays conducted under the US National Toxicology Program, with the NOAEL and the BMDL calculated from these same datasets using the Hill model to describe the observed dose-response relationships. Most of these studies follow a relatively common protocol, with male and female rats and mice tested at a control and three dose levels for each test agent (Sand et. al., 2011) [10].

Signal-to-noise crossover dose is the dose that produces a predetermined ratio of additional risk to "background noise" - length of the two-sided 90\% confidence interval of response rate at that dose (known as signal-to-noise response, SNR). The traditional SNR values are 1 and $2 / 3$.

$$
S N R=\frac{P(d)-P(0)}{P 95-P 05}
$$

\section{$1.2 \quad$ Outline}

The introduction of the SNCD by Sand et al. (2011) [18] did not address uncertainty in this statistic, in part because of the use of the difference between the $95^{\text {th }}$ and $5^{\text {th }}$ percentile as a measure of uncertainty in the biological signal. The overarching objective of this thesis is to address this limitation, by developing confidence limits on the SNCD, similar to those developed for the BMD. The Hill model is chosen to represent the dose-response relationship in this research because of its relative simplicity and high degree of flexibility in describing diverse dose-response shapes.

In chapter 2, the maximum likelihood estimates (MLEs) and the variancecovariance matrix of MLEs of the Hill model parameters are assessed. Estimation of BMD as a function of the MLEs and variance-covariance matrix of the estimated Hill model parameters are described. A detailed simulation study is conducted to evaluate the accuracy and precision of the MLEs of the Hill model parameters under a variety of dose-response shapes.

Chapter 3 introduces an analytical approach to develop upper and lower con- 
fidence limits on the SNCD under Hill model. Parametric and non-parametric bootstrap methods for obtaining upper and lower confidence bounds on the SNCD are also presented. We develop expressions for the bias and variability of estimates of the SNCD, as well as the coverage probability of the confidence bounds on the SNCD as a function of Hill model parameters, the number of dose levels, and the number of trials per dose group. Five different methods of estimating the analytical bias is presented and compared to get the best method to estimate the bias.

Chapter 4 uses the analytical method developed in chapter 2 and 3, to estimate the PoDs such as BMD, SNCD and SNCDL; and the traditional profile likelihood method to estimate BMDL for datasets obtained from NTP data base. Comparison of BMD to SNCD and BMDL to SNCDL as well as estimation of extra risk at all estimated SNCD and SNCDL are calculated and presented.

Finally, conclusions and directions for future research are presented in chapter 5. 


\section{BIBLIOGRAPHY}

[1] Barnes.D.G. and Dourson.M. (1988) Reference dose (RfD): description and use in health risk assessments, Regulatory Toxicology and Pharmacology $8: 471-486$

[2] Klassen, Curtis D., Casarett,Louis J., and ebrary, Inc (2008) Casarett and Doull's Toxicology: the basic science of poisons, Fundam Appl Toxicol $4: 854-871$.

[3] Committee on the Institutional Means for Assessment of Risks to Public Health, Commission of Life Sciences, National Research Council (1983) Risk Assessment in the Federal Government: Managing the Process, National Academy Press, Washington.D.C. 1983

[4] Crump,K., (1984) A new method for determining allowable daily intakes, New York: London: McGraw-Hill Medical: McGraw-Hill (distribution) 2008.

[5] EFSA European Food Safety Authority, http://www.efsa.europa.eu/

[6] EFSA 2005 Opinion of the Scientific Committee on a request from EFSA related to a harmonised approach for risk assessment of substances which are both genotoxic and carcinogenic, The EFSA Journal 282:1-31

[7] Krewski.D., Brand.K.P., Burnett.R.T., and Zielinski.J.M. (2002) Simplicity versus complexity in the development of risk models for dose-response assessment. Human Ecological Risk Assessment, 8, 1355-1374

[8] Krewski.D., Westphal.M., Anderson.M.E., Paoli.G.M., Chiu.W.A. AlZoughool.M., Croteau.M.C., Burgoon.L.D., and Cote.I. (2014) A frame- 
work for the next generation of risk science, Environmental Health Perspectives. 122. $796-805$

[9] Lehman,A.J., and Fitzhugh,O.G. 1954 100-fold margin of safety, Assoc.Food Drug Off. U.S.Q. Bull. 1954;18:33-35

[10] Sand.S., Portier.C.J., and Krewski.D., (2011) A Signal-to-Noise Crossover Dose as the Point of Departure for Health Risk Assessment, Environ Health Perspect 119:1766-1774

[11] US EPA United States Environmental Protection Agency, http://www.epa.gov/ 


\section{STATISTICAL PROPERTIES OF HILL MODEL PARAMETER AND BENCHMARK DOSE ESTIMATES}

\section{$2.1 \quad$ Introduction}

Risk assessments are conducted for a variety of health effects that may result from exposure to environmental or physical agents or situations that may affect human health or contribute to environmental hazards [23]. Health risk assessments often include an analysis of the dose-response relationship between exposure and health-related outcomes. No-observed-adverse-effect-level/lower-observedadverse-effect level (NOAEL/LOAEL) was originally introduced by Lehman and Fitzhugh (1954) [14] as a point of departure (PoD) for establishing human exposure guidelines. While the NOAEL has been used for many years in the dose-response assessment, it has recognized limitations. Since the introduction of the benchmark dose (BMD) by Crump (1984) [3], there has been considerable discussion about its merits relative to the traditional NOAEL.

The NOAEL has many limitations compared to the BMD. In particular, not all of the available data are used to determine the NOAEL, and its value is restricted to one of the experimental dose levels (Gaylor et. al. 1998) [15]. Thus the NOAEL is strongly dependent on the choice of dose levels made, and on the spacing between these levels; for example, if the dose levels are widely-spaced, the true NOAEL may be considerably higher than that estimated using the data [8]. Further, simulation and empirical studies have also been used to demonstrate the sensitivity of the NOAEL to sample size, and its high variability from experiment to experiment.

Leisenring et. al. (1992) [22] investigated the impact of the shape of the underlying dose-response curve (using Weibull models) on the distribution of the NOAEL and confirmed that the average risk level associated with the NOAEL may be sub- 
stantial. Moreover, the NOAEL is not appropriate for substances that are genotoxic and carcinogenic [8]. An extensive retrospective investigation of a large database for developmental toxicity demonstrates the effectiveness of the benchmark dose approach to remove some of the ambiguity in selecting a NOAEL/LOAEL, and to avoid the wide discrepancies in risk that can occur at the NOAEL/LOAEL (Allen et. al. 1994a) [1].

Kimmel (1993) [9] presented several alternatives to the NOAEL approach including the BMD for risk assessment of reproductive hazards. Murrell et. al. (1998) [15] performed a critical assessment of the BMD and concluded that the BMD defined in terms of extra risk has consistent relationship with model parameters characterizing the dose-response relationship. Foster et. al. (1995) [11] discussed the use of the BMD method in developmental toxicity studies and concluded that it offers significant advantages. Calculation of a NOAEL generally utilizes the data that are categorized into distinct dose groups. However, in some studies, including most epidemiological studies, categorization of subjects into dose groups is an arbitrary process. Such categorization is not required in the benchmark dose approach (Slikker et. al. 1996)[19]. To estimate the potency of carcinogens, comparison of the European Union (EU) T25 was accomplished with the benchmark dose by Van Landingham et. al. (2001) [22]. They concluded that the EU T25 method underestimated the true T25 dose, and overestimated the carcinogenic potency, but the BMD estimate is less biased and has less variation about the true T25 value than the EU estimate.

With the goal of reducing the number of animals used in experiments, Oberg (2010) [16] suggested unequal group sizes for experiments with fewer animals in the high response dose groups and more in those that were closer to toxicological thresholds. The need to evaluate how the total number of animals affects the quality of the estimate of the BMD was also discussed.

It is widely agreed that the characterization and quantification of potential risks 
at human exposure levels can be improved if full use is made of the dose-response curve that typically arises from data based on animals. Essentially, one wishes to model the probability or odds of a response as a function of dose levels. Sufficient data are required to enable both accurate and precise characterization of the doseresponse relationship (USEPA 2012)[21].

Many models have been used to represent the dose-response relationship. These include models from the linear, linear threshold, exponential, Hill, logistic, probit, loglogistic, log-probit, Weibull, gamma and linearized multistage families. Some of the models described by standard probability distribution functions such as the logistic, log-logistic, probit and log-probit, Hill and Weibull are based on the notion that each subject in the population has its own tolerance to a chemical; on the other hand, the multistage and gamma model are considered to be stochastic models that are based on the idea that a positive response is the result of the random occurrence of one or more biological events (Krewski et. al. 1981 and Casarett et. al. 1996) [11] and [3].

Provided that the model fitting is performed satisfactorily, statistically reliable estimates of the PoD can be obtained using the BMD (EFSA 2005) [8]. No particular assumptions are needed about the nature of toxicological dose-responses, other than that the change in response generally does not decrease with higher doses. The BMD is based on setting the benchmark response (BMR) to a prescribed level. There are two general definitions of the BMR at a given dose level. One is based on the notion of additional risk, and is simply described as the difference between the responding fraction at the given dose and that in the control group. The other is based on extra risk, and characterized by the ratio of this difference to the fraction of non responding subjects in the control group. Regardless of the definition, the BMD is the dose that produces a predetermined change in the BMR. Commonly chosen values for this specified change are $1 \%, 5 \%$ and $10 \%$.

In this chapter, we propose an approach for determining point and interval es- 
timates for the benchmark dose. We use a three-parameter Hill model to describe the dose-response relationship. Clearly, the ability of the proposed methodology to estimate the BMD will depend upon how well the Hill model parameters are estimated. We conduct and report the results of a large scale simulation study designed to evaluate the performance of the suggested technique for BMD estimation under a wide array of situations distinguished by different dose response relationships, number of dose levels, and number of trials per dose. We consider both the additional and extra risk definitions of the BMD with a number of different benchmark responses.

\subsection{Hill Model Characterization of the Dose-Response Relationship}

\subsubsection{Model Specification}

The three parameter Hill model has been commonly used to describe doseresponse relationships with a nonzero background incident. Suppose that a survival study experiment has been conducted where $n_{i}$ subjects have been exposed to a dose level, $d_{i}, i=1, \cdots, k$. The number of subjects, $x_{i}$, who respond at a dose $d_{i}$ are recorded. The Hill model describes the probability of a response from a subject exposed to a dose $d_{i}$ according to:

$$
P\left(d_{i}\right)=\alpha+(1-\alpha)\left(\frac{d_{i}^{\eta}}{\kappa^{\eta}+d_{i}^{\eta}}\right)
$$

where $\alpha, \kappa$ and $\eta$ are parameters that characterize the dose-response relationship. Generally speaking, $\alpha$ is considered to be a background incident parameter. Note that when $d_{i}=0, P\left(d_{i}\right)=\alpha$. In addition, if the background incident rate is zero, the three parameter Hill model reduces to a two-parameter logistic regression model. Specifically, setting $\alpha=0$ in equation 2.1 allows us to write that

$$
\operatorname{logit}\left[P\left(d_{i}\right)\right]=\ln \left[\frac{P\left(d_{i}\right)}{1-P\left(d_{i}\right)}\right]=-\eta \ln (\kappa)+\eta \ln \left(d_{i}\right)=-\beta_{0}+\beta_{1} d_{i}^{*}
$$

where $\beta_{0}=\eta \ln \kappa, \beta_{1}=\eta$ and $d_{i}^{*}=\ln d_{i}$. The parameters $\kappa$ and $\eta$ are location and 
shape parameters, respectively. We illustrate their impact in characterizing the dose response relationship in section 2.2.2.

\subsubsection{Dose-Response Characteristics of the selected Hill Model}

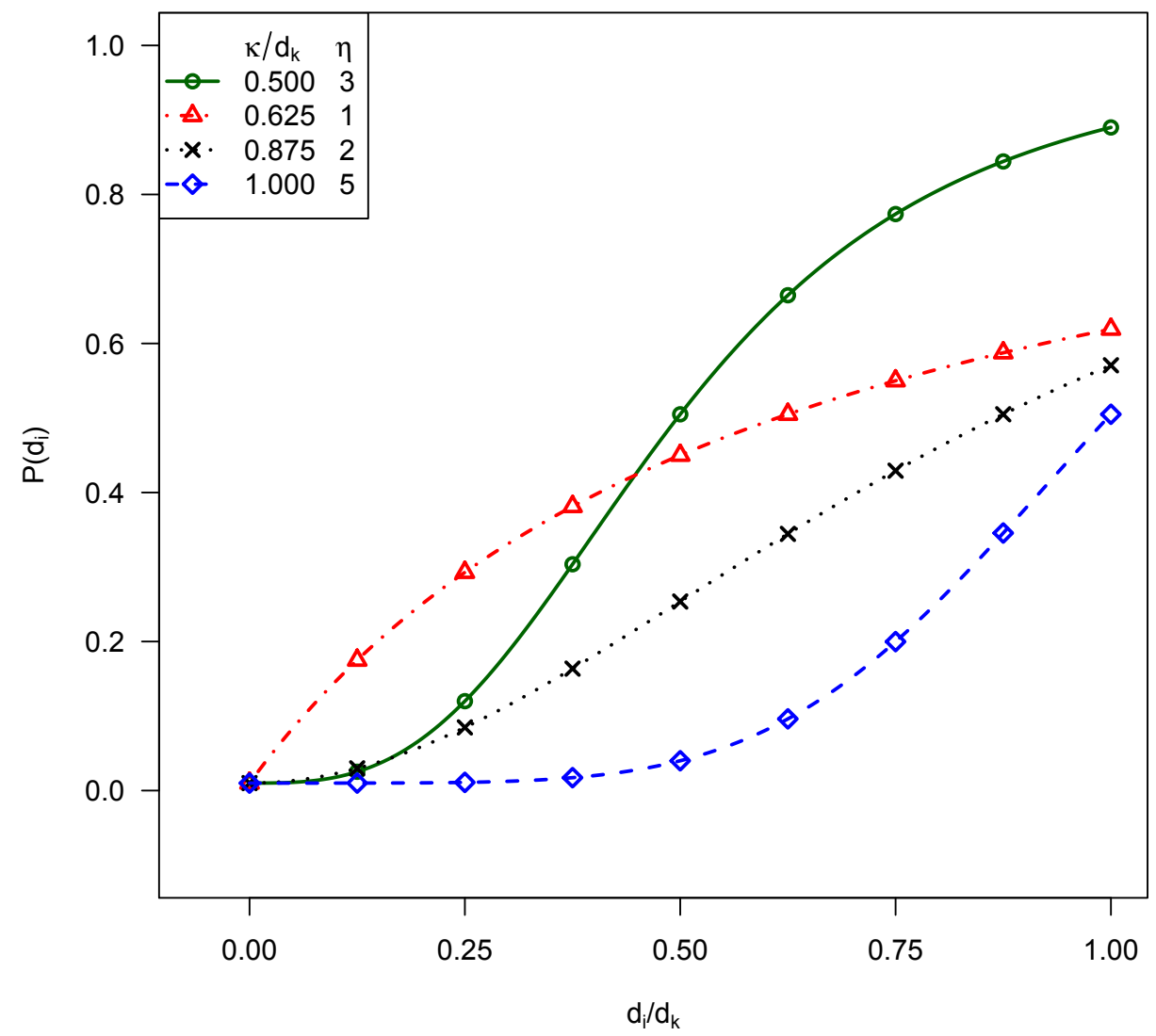

Figure 2.1: Dose Response Curves for Selected Hill Model Parameters $\kappa$ and $\eta$ with $\alpha=0.01$

The characteristics associated with a dose-response curve described by the Hill model through to the maximum dose level, $d_{k}$ can be quite varied. A number of examples are presented in Figure 2.1. The value of the background parameter, $\alpha$, is set to 0.01 in all cases (suggesting that $P(0)=0.01$ for all curves), while different values are specified for the location and shape parameters. Expanding on Figure 2.1, 
each panel of Figure 2.2 characterizes, for a different value of the shape parameter, $\eta$, the response curve over the range of dose levels specified in the experiment. The shape parameter is varied from a value of one in panel (a) through to a value of five in panel (d). Since $\kappa$ is a location parameter, it can be viewed relative to the maximum dose level, $d_{k}$, which, for purposes of illustration, is set to one in Figures 2.1 and 2.2. For each panel in the latter figure, seven different curves are plotted for values of the ratio $\kappa / d_{k}$ ranging from 0.50 to 1.25 . Note that the smaller this ratio, the steeper the curve representing the dose response relationship, suggesting that the probability of a response grows at a faster rate as this ratio $\kappa / d_{k}$ gets smaller for specific value of $\eta$.

Returning to the shapes associated with the curves in each of the four panels of Figure 2.2, it can be seen in panel (a) that when $\eta$ is set to one, the shape of the dose response relationship is generally convex over the range of dose levels used. In addition, provided that the ratio $\kappa / d_{k}$ is in the range of 0.5 to 1.25 , the probability of a response stays in the range of 0.45 to 0.65 , even at the maximum dose. Note also that the probability of a response is higher for smaller values of this ratio. In panel (b), the shape parameter is set to two. In this instance, the dose response relationship is concave over the range of dose levels when $\kappa / d_{k}$ is relatively small, and begins to resemble an S-shaped curve more and more as this ratio increases. At the maximum dose level, when compared to panel (a), the possible values for the probability of a response are (from 0.4 to 0.8 ) more varied over the same range of ratios of $\kappa / d_{k}$. In panels (c) and (d), in which the shape parameter is set to three and five, respectively, the spectrum of curves is similar to that illustrated in panel (b), ranging from concave to S-shaped as the value of the ratio $\kappa / d_{k}$ increases. However, when comparing panels (b) through (d), it can be seen that the curves become steeper as the value of the shape parameter increases. In addition, the ranges associated with the probability of response at the maximum dose increase with increasing shape parameter $\eta$. Specifically, the probability of response ranges 0.3 to 0.9 at the maximum dose 


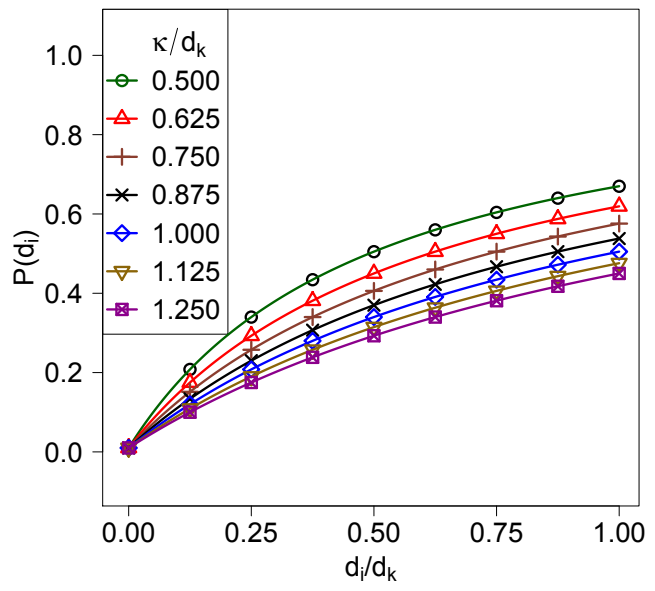

(a) $\eta=1$

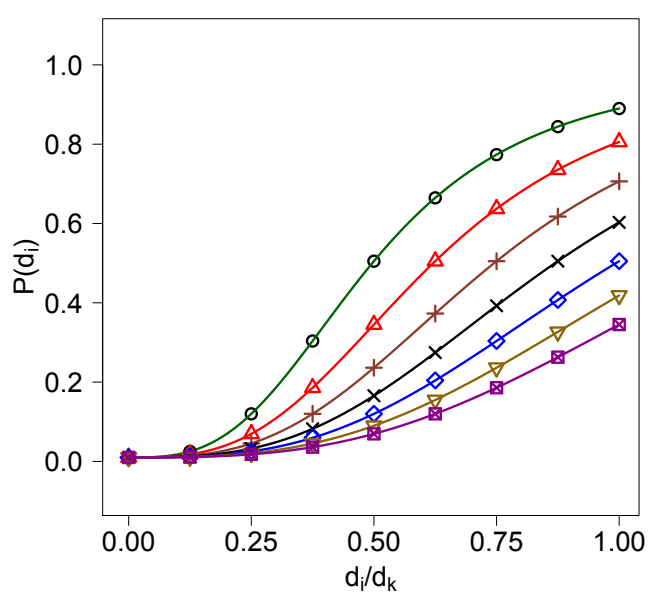

(c) $\eta=3$

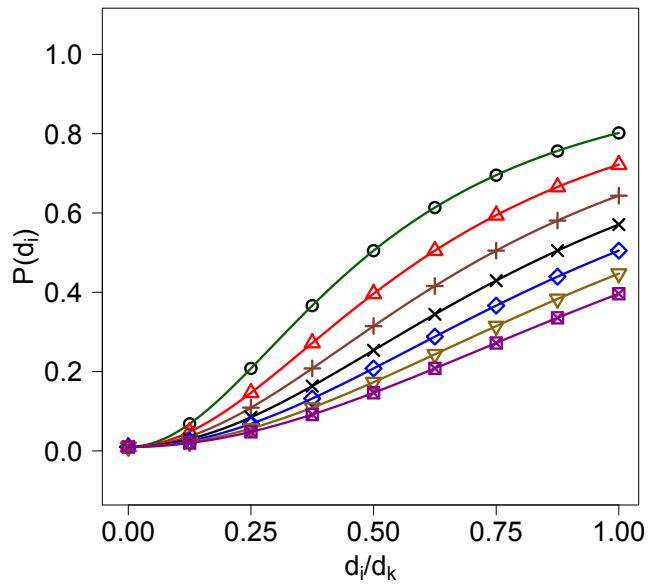

(b) $\eta=2$

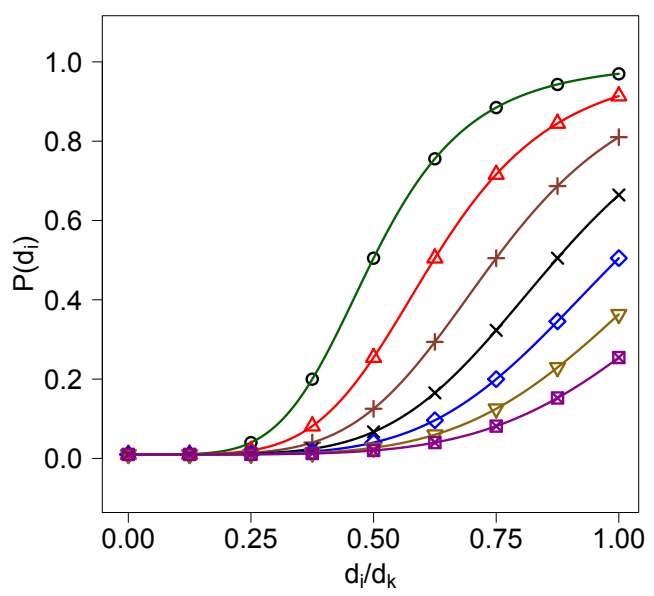

(d) $\eta=5$

Figure 2.2: Dose Response for $\eta=(1,2,3,5)$ for $\kappa / d_{k}=(0.50-1.25)$

level when $\eta=3$ and spans 0.25 to 0.95 when $\eta=5$. Dissimilarity in the response curve shape over the range of $\kappa / d_{k}$ increases with increasing shape parameter value (from one to five). Responses are the most homogeneous over the range of $\kappa / d_{k}$ when shape parameter is one.

Figure 2.3 presents a different representation of the various curves plotted in Figure 2.2. Specifically, each of the four panels in Figure 2.3 describe the doseresponse relationships over the range of dose levels specified in an experiment for fixed 


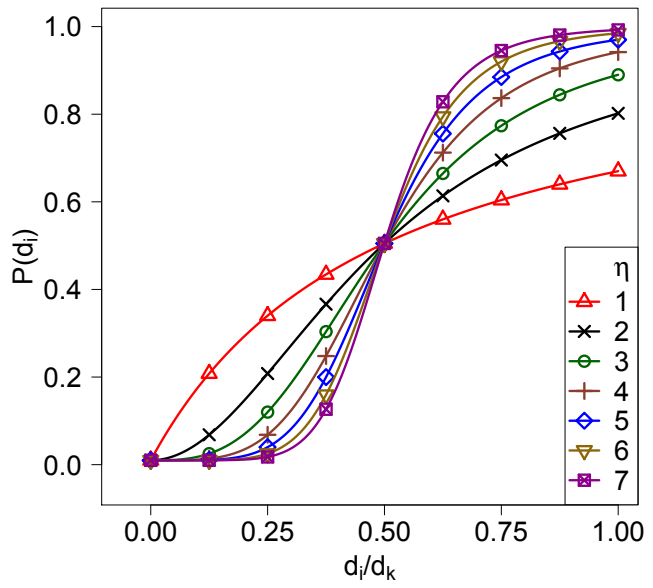

(a) $\kappa / d_{k}=0.500$

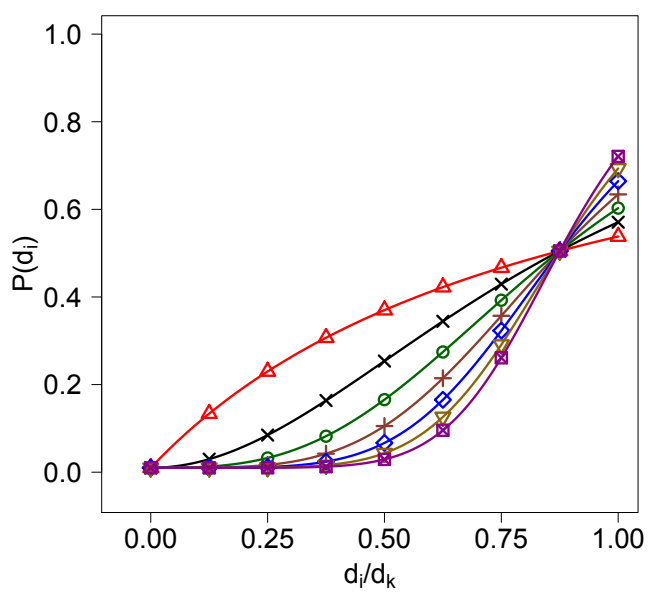

(c) $\kappa / d_{k}=0.875$

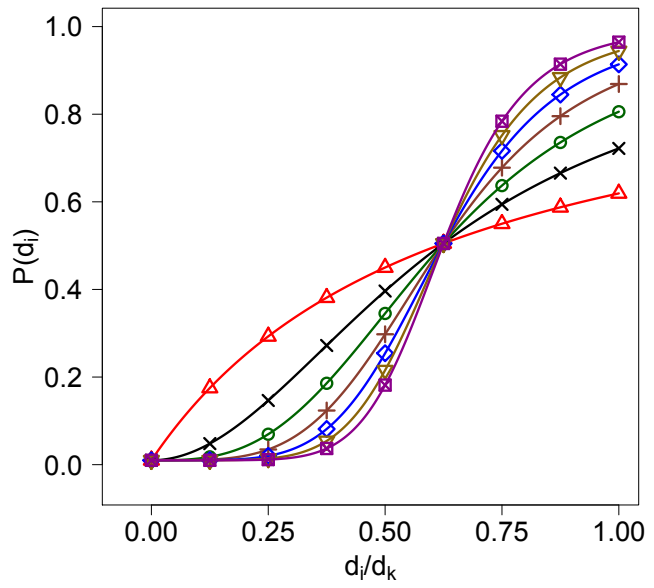

(b) $\kappa / d_{k}=0.625$

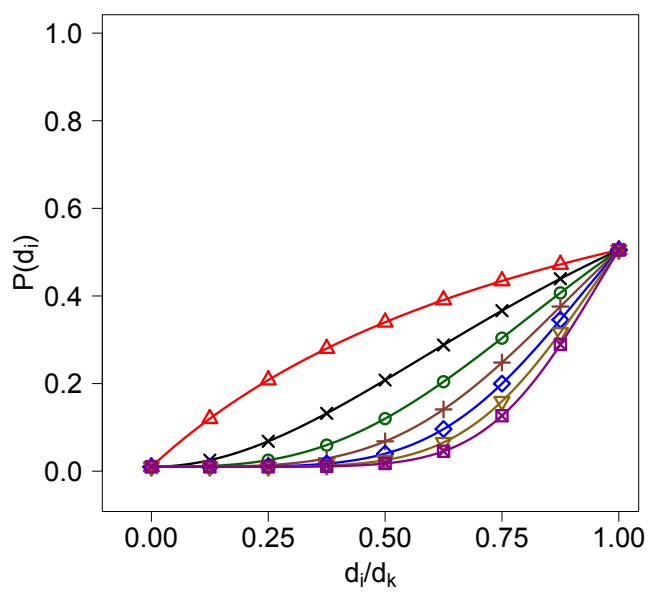

(d) $\kappa / d_{k}=1.000$

Figure 2.3: Dose Response for $\kappa / d_{k}=(0.500,0.625,0.875,1.00)$ for $\eta=(1,2,3,4$, $5,6,7)$

values of the ratio $\kappa / d_{k}$, for different values of the shape parameter ranging from one through seven. As evidenced in panels (a) through (d) in Figure 2.3, dissimilarity in the response curves decreases as the value of the shape parameter increases when $\kappa / d_{k}$ gets larger. Response curves are the most homogeneous over shape parameter values of two to seven when $\kappa / d_{k}$ is close to or greater than one. 


\subsection{Point of Departure: Benchmark Dose}

As stated earlier, the focus of this chapter is on the estimation of the measure for point of departure known as the benchmark dose, BMD, where the dose-response relationship is described by the three-parameter Hill model. Specifically, we seek to develop statistically reliable point and interval estimates for the BMD. The benchmark dose is defined as the dose that produces a predetermined difference in the response rate of an adverse effect (otherwise known as the benchmark response, BMR) compared to the background rate, or a predetermined ratio of this difference to the proportion of subjects that do not exhibit a response in the control group. The former of these two definitions of the BMD is based on the notion of additional risk. It can be thought as the dose, $d_{a}$, which yields a specified benchmark response, BMR, according to

$$
B M R=P\left(d_{a}\right)-P(0)
$$

The latter definition is based on the idea of extra risk, and is the dose $d_{e}$, which yields a specified BMR using the equation

$$
B M R=\frac{P\left(d_{e}\right)-P(0)}{1-P(0)}
$$

Traditional values selected for the BMR in the above two expressions are $1 \%, 5 \%$, and $10 \%$. In what follows here, the probability of a response at dose level $\mathrm{d}, \mathrm{P}(\mathrm{d})$, will be described according to a three-parameter Hill model as defined in equation 2.1. Obviously, the ability to appropriately estimate the benchmark dose will be highly dependent on how well the Hill model parameters are estimated from experimental data. We shall investigate this via an extensive simulation study. However, prior to doing so, we first present the methodology proposed to develop both point and interval estimates for the Hill model parameters and the benchmark dose. 


\subsection{Estimation of Hill Model Parameters and Benchmark Dose}

In this section, we present the development of point and interval estimates for the parameters in the Hill model and the benchmark dose that are based on maximum likelihood estimation.

\subsubsection{Hill Model Parameter Estimates}

Suppose that an experiment has been conducted where $n_{i}$ independent subjects have been exposed to a dose level, $d_{i}, \mathrm{i}=1, \cdots, \mathrm{k}$, where the first of these $\mathrm{k}$ groups is a control with $d_{1}=0$. For each group, the number of subjects, $x_{i}$, who respond at dose $d_{i}$ is recorded. If $P\left(d_{i}\right)$ is the probability for response at dose $d_{i}$, the Likelihood function of the data is given by

$$
L=\prod_{i=1}^{k}\left(P\left(d_{i}\right)\right)^{x_{i}}\left(1-P\left(d_{i}\right)\right)^{\left(n_{i}-x_{i}\right)}
$$

while the log-likelihood can be written as

$$
\mathcal{L}=\sum_{i=1}^{k}\left\{x_{i} \ln \left[P\left(d_{i}\right)\right]+\left(n_{i}-x_{i}\right) \ln \left[1-P\left(d_{i}\right)\right]\right\}
$$

If $P\left(d_{i}\right)$ is described by the three-parameter Hill model in 2.1 , the expression for the log-likelihood can be written as

$$
\mathcal{L}=\sum_{i=1}^{k}\left\{x_{i} \ln \left(\alpha+(1-\alpha)\left(\frac{d_{i}^{\eta}}{\kappa^{\eta}+d_{i}^{\eta}}\right)\right)+\left(n_{i}-x_{i}\right) \ln \left((1-\alpha)\left(\frac{\kappa^{\eta}}{\kappa^{\eta}+d_{i}^{\eta}}\right)\right)\right\}
$$

Maximum likelihood estimates of $\alpha, \kappa$ and $\eta$ are determined by setting the first order partial derivatives of the log-likelihood with respect to $\alpha, \kappa$ and $\eta$ to zero and solving to yield $\widehat{\alpha}, \widehat{\kappa}$ and $\widehat{\eta}$. These equations are given by

$$
\begin{gathered}
\frac{\partial \mathcal{L}}{\partial \alpha}=\sum_{i=1}^{k}\left\{\left(\frac{x_{i} \kappa^{\eta}}{\alpha \kappa^{\eta}+d_{i}^{\eta}}\right)-\left(\frac{n_{i}-x_{i}}{1-\alpha}\right)\right\} \\
\frac{\partial \mathcal{L}}{\partial \kappa}=\sum_{i=1}^{k}\left\{\left(\frac{\eta d_{i}^{\eta} n_{i}}{\kappa\left(d_{i}^{\eta}+\kappa^{\eta}\right)}\right)-\left(\frac{\eta d_{i}^{\eta} x_{i}}{\kappa\left(\alpha \kappa^{\eta}+d_{i}^{\eta}\right)}\right)\right\}
\end{gathered}
$$




$$
\frac{\partial \mathcal{L}}{\partial \eta}=\sum_{i=1}^{k}\left\{d_{i}^{\eta}\left(\ln d_{i}-\ln \kappa\right)\left(\left(\frac{x_{i}}{\alpha \kappa^{\eta}+d_{i}^{\eta}}\right)-\left(\frac{n_{i}}{\kappa^{\eta}+d_{i}^{\eta}}\right)\right)\right\}
$$

The negative of the inverse of the matrix of second derivatives evaluated at $\widehat{\alpha}, \widehat{\kappa}$ and $\widehat{\eta}$ serves as an approximate estimated asymptotic covariance matrix. The second order derivatives are as follows:

$$
\begin{gathered}
\frac{\partial^{2} \mathcal{L}}{\partial \alpha^{2}}=-\sum_{i=1}^{k}\left\{\frac{x_{i} \kappa^{2 \eta}}{\left(\alpha \kappa^{\eta}+d_{i}^{\eta}\right)^{2}}-\frac{\left(n_{i}-x_{i}\right)}{(1-\alpha)^{2}}\right\} \\
\frac{\partial^{2} \mathcal{L}}{\partial \kappa^{2}}=\sum_{i=1}^{k}\left\{\left(\frac{\eta d_{i}^{\eta}}{\kappa^{2}}\right)\left(\frac{x_{i}\left(\alpha \kappa^{\eta}(\eta+1)+d_{i}^{\eta}\right)}{\left(\alpha \kappa^{\eta}+d_{i}^{\eta}\right)^{2}}-\frac{n_{i}\left(\kappa(\eta+1)+d_{i}^{\eta}\right)}{\left(\kappa^{\eta}+d_{i}^{\eta}\right)^{2}}\right)\right\} \\
\frac{\partial^{2} \mathcal{L}}{\partial \eta^{2}}=\sum_{i=1}^{k}\left\{\kappa^{\eta} d_{i}^{\eta}\left(\ln d_{i}-\ln \kappa\right)^{2}\left(\frac{x_{i} \alpha}{\left(\alpha \kappa^{\eta}+d_{i}^{\eta}\right)^{2}}-\frac{n_{i}}{\left(\kappa^{\eta}+d_{i}^{\eta}\right)^{2}}\right)\right\} \\
\frac{\partial^{2} \mathcal{L}}{\partial \alpha \partial \kappa}=\sum_{i=1}^{k}\left\{\frac{\eta x_{i} d_{i}^{\eta} \kappa^{(\eta-1)}}{\left(\alpha \kappa^{\eta}+d_{i}^{\eta}\right)^{2}}\right\} \\
\frac{\partial^{2} \mathcal{L}}{\partial \alpha \partial \eta}=\sum_{i=1}^{k}\left\{\frac{x_{i} \kappa^{\eta} d_{i}^{\eta}\left(\ln \kappa-\ln d_{i}\right)}{\left(\alpha \kappa^{\eta}+d_{i}^{\eta}\right)^{2}}\right\} \\
k \sum_{i=1}^{k}\left\{\left(\eta \kappa^{(\eta-1)} d_{i}^{\eta}\right)\left(\ln d_{i}-\ln \kappa\right)\left(\frac{n_{i}}{\left(d_{i}^{\eta}+\kappa^{\eta}\right)^{2}}-\frac{\alpha x_{i}}{\left(\alpha \kappa^{\eta}+d_{i}^{\eta}\right)^{2}}\right)\right\}
\end{gathered}
$$

The maximum likelihood estimates of Hill model parameters are obtained using mle2 function from bbmle package in R-statistical software. In the simulation studies, initial values for $\kappa$ is selected to be in the range from one to $25, \eta$ from one to 10 and $\alpha$ from 0.001 to 0.999 . In mle2 function, finite difference approximations of derivatives are implemented to obtain maximum likelihood estimates and their variance-covariance estimates.

In our preliminary analysis, the R-outputted variance-covariance matrix is compared with variance-covariance matrix obtained by negating the inverse of the matrix of second derivatives evaluated at the maximum likelihood estimates when number of dose levels is four and numbers of trials per dose are of 25, 50, 100, 200, 300, 400 and 500. We observe negative variances for the latter while number of trials per dose 
is of 25 , but the estimates from these two approaches start to converge at 100 trials per dose.

\subsubsection{Estimation of Benchmark Dose}

Suppose we wish to obtain an estimator of the benchmark dose, $\widehat{B M D} D$, for a specified benchmark response, BMR, using the estimates of the Hill model described in the previous sub-section. We also wish an estimate of the variability in this estimator. Initially, consider the expression for extra risk given in 2.4. Replacing $d_{e}$ in this equation with $\widehat{B M D}$ allow us to write that

$$
B M R=\frac{P(\widehat{B M D})-P(0)}{1-P(0)}
$$

From the estimated Hill model counterpart to equation 2.1

$$
P(\widehat{B M D})=\widehat{\alpha}+(1-\widehat{\alpha})\left(\frac{\widehat{B M D} D^{\widehat{\eta}}}{\widehat{\kappa}^{\widehat{\eta}}+\widehat{B M D} D^{\widehat{\eta}}}\right)
$$

$P(0)=\widehat{\alpha}$. Substituting into 2.17 and simplifying yields

$$
B M R=\left(\frac{\widehat{B M D} D^{\widehat{\eta}}}{\widehat{\kappa}^{\widehat{\eta}}+\widehat{B M D} D^{\widehat{\eta}}}\right)
$$

Solving for $\widehat{B M D}$ gives

$$
\widehat{B M D}=\widehat{\kappa}\left[\frac{B M R}{1-B M R}\right]^{\frac{1}{\hat{\eta}}}=\widehat{h}_{1}
$$

Note that this expression only depends on $\widehat{\kappa}$ and $\widehat{\eta}$ (and BMR), but not on the estimate of the background parameter, $\widehat{\alpha}$.

An expression for the variance of $\widehat{B M D}$ can be obtained by taking a first order multivariate Taylor Series expansion of $\widehat{B M D}$ about $\widehat{\kappa}$ and $\widehat{\eta}$. If we define $\beta=(\kappa, \eta)^{\prime}$ and $\widehat{\beta}=(\widehat{\kappa}, \widehat{\eta})^{\prime}$, this expression can be written as

$$
\widehat{B M D} \approx B M D+\left.\Phi_{\widehat{\kappa}}\right|_{\widehat{\beta}=\beta}(\widehat{\kappa}-\kappa)+\left.\Phi_{\widehat{\eta}}\right|_{\widehat{\beta}=\beta}(\widehat{\eta}-\eta)
$$


where BMD is the true unknown benchmark dose,

$$
\begin{aligned}
\Phi_{\widehat{\kappa}} & =\left.\frac{\partial \widehat{h}_{1}}{\partial \widehat{\kappa}}\right|_{\widehat{\beta}=\beta}=\left(\frac{B M R}{1-B M R}\right)^{\frac{1}{\eta}} \\
\Phi_{\widehat{\eta}} & =\left.\frac{\partial \widehat{h}_{1}}{\partial \widehat{\eta}}\right|_{\widehat{\beta}=\beta}=-\frac{\kappa}{\eta^{2}}\left(\frac{B M R}{1-B M R}\right)^{\frac{1}{\eta}} \ln \left(\frac{B M R}{1-B M R}\right)
\end{aligned}
$$

Taking the variance of 2.21 allows us to write that

$$
\operatorname{Var}(\widehat{B M D}) \approx \Phi_{\kappa}^{2} \operatorname{Var}(\widehat{\kappa})+\Phi_{\eta}^{2} \operatorname{Var}(\widehat{\eta})+2 \Phi_{\kappa} \Phi_{\eta} \operatorname{Cov}(\widehat{\kappa}, \widehat{\eta})
$$

which can be estimated by

$$
\widehat{\operatorname{Var}}(\widehat{B M D}) \approx \widehat{\Phi}_{\kappa}^{2} \widehat{\operatorname{Var}}(\widehat{\kappa})+\widehat{\Phi}_{\eta}^{2} \widehat{\operatorname{Var}}(\widehat{\eta})+2 \widehat{\Phi}_{\kappa} \widehat{\Phi}_{\eta} \widehat{\operatorname{Cov}}(\widehat{\kappa}, \widehat{\eta})
$$

where the estimated variances and covariances of $\widehat{\kappa}$ and $\widehat{\eta}$ are available from the estimated asymptotic covariance matrix of the estimates of the Hill model parameters, and $\widehat{\Phi}_{\widehat{\kappa}}$ and $\widehat{\Phi}_{\widehat{\eta}}$ are computed by replacing $\kappa$ and $\eta$ in $\Phi_{\widehat{\kappa}}$ and $\Phi_{\widehat{\eta}}$ with $\widehat{\kappa}$ and $\widehat{\eta}$.

Provided that we ignore any bias in the estimator $\widehat{B M D} D$, the Delta method allows us to propose the following $100(1-\alpha) \%$ confidence interval for BMD based on the extra risk definition:

$$
\widehat{B M D} \pm Z_{1-\alpha / 2} \sqrt{\widehat{\operatorname{Var}}(\widehat{B M D})}
$$

where $Z_{1-\alpha / 2}$ is the $100(1-\alpha / 2)^{\text {th }}$ percentile of a standard normal distribution.

The confidence interval in 2.26 can also be used for the BMD defined by 2.3 in terms of additional risk. However, in this case, using the same development as above for extra risk, the point estimator for BMD is

$$
\widehat{B M D}=\widehat{\kappa}\left[\frac{B M R}{1-\widehat{\alpha}-B M R}\right]^{\frac{1}{\hat{\eta}}}=\widehat{h}_{2}
$$

and estimate of the variance of BMD is

$$
\begin{aligned}
\widehat{\operatorname{Var}}(\widehat{\operatorname{BMD}}) \approx & \widehat{\Phi}_{\alpha}^{2} \widehat{\operatorname{Var}}(\widehat{\alpha})+\widehat{\Phi}_{\kappa}^{2} \widehat{\operatorname{Var}}(\widehat{\kappa})+\widehat{\Phi}_{\eta}^{2} \widehat{\operatorname{Var}}(\widehat{\eta}) \\
& +2 \widehat{\Phi}_{\alpha} \widehat{\Phi}_{\kappa} \widehat{\operatorname{Cov}}(\widehat{\alpha}, \widehat{\kappa})+2 \widehat{\Phi}_{\alpha} \widehat{\Phi}_{\eta} \widehat{\operatorname{Cov}}(\widehat{\alpha}, \widehat{\eta})+2 \widehat{\Phi}_{\kappa} \widehat{\Phi}_{\eta} \widehat{\operatorname{Cov}}(\widehat{\kappa}, \widehat{\eta})
\end{aligned}
$$


where

$$
\begin{gathered}
\Phi_{\alpha}=\left.\frac{\partial \widehat{h}_{2}}{\partial \widehat{\alpha}}\right|_{\widehat{\beta}=\beta}=\frac{\kappa(B M R)^{\frac{1}{\eta}}}{\eta(1-\alpha-B M R)^{\left(1+\frac{1}{\eta}\right)}} \\
\Phi_{\kappa}=\left.\frac{\partial \widehat{h}_{2}}{\partial \widehat{\kappa}}\right|_{\widehat{\beta}=\beta}=\left(\frac{B M R}{1-B M R-\alpha}\right)^{\frac{1}{\eta}} \\
\Phi_{\eta}=\left.\frac{\partial \widehat{h}_{2}}{\partial \widehat{\eta}}\right|_{\widehat{\beta}=\beta}=-\frac{\kappa}{\eta^{2}}\left(\frac{B M R}{1-B M R-\alpha}\right)^{\frac{1}{\eta}} \ln \left(\frac{B M R}{1-B M R-\alpha}\right)
\end{gathered}
$$

\subsection{Simulation Study}

In order to assess the inference procedures proposed here for the BMD that are based on a three parameter Hill model, a large scale simulation study is conducted. We also investigate the conditions under which the Hill model parameters are wellestimated, as there would be repercussions on the point and interval estimates of the BMD when this may not be the case.

We simulate 1000 datasets for each of the thirty-two different scenarios that are distinguished by the dose-response relationship, number of dose levels, and the number of trials per dose. With respect to the number of dose levels, values of four $\left(d_{i} / \kappa=0,0.25,0.50,1.0\right)$ and $\operatorname{six}\left(d_{i} / \kappa=0,0.25,0.375,0.5,0.75,1.0\right)$ are considered; for both cases $d_{k}=8$. As the focus of this research is in the lower dose levels, three out of four and four out of six dose points are chosen in the lower half of the dose range respectively for four and six dose levels. We set the number of trials at each of the dose levels, either $n_{i}=50,100,200$ or 500. Finally, four different dose response relationships are investigated; specifically, one curve is selected from each of four panels in Figure 2.2. From panel (a) where $\eta=1$, we choose $\kappa / d_{k}$ $=0.625$ and from panel (b) where $\eta=2$, we select $\kappa / d_{k}=0.875$. We opt for $\kappa / d_{k}=0.5$ when $\eta=3$ as in panel (c), and $\kappa / d_{k}=1$ when $\eta=5$ in panel (d). For all four curves, we set the background parameter $\alpha=0.01$. Thus, the four doseresponse relationships are described by the Hill model in Figure 2.1 with the following 
parameters; (i) $\alpha=0.01, \eta=1, \kappa=5$ (ii) $\alpha=0.01, \eta=2, \kappa=7$ (iii) $\alpha=0.01, \eta=$ $3, \kappa=4$ (iv) $\alpha=0.01, \eta=5, \kappa=8$.

To generate a single simulated dataset for each of the thirty-two combinations considered, we use the Hill model in equation 2.1 to calculate $P\left(d_{i}\right)$ at each dose level, $d_{i}$ since $\alpha, \eta$ and $\kappa$ are specified. Then at each dose level, $d_{i}$, we generate $n_{i}$ Bernoulli random variables with parameter $P\left(d_{i}\right)$. These Bernoulli outcomes serve as one of the 1000 simulated datasets associated with one of the thirty-two conditions investigated here that are distinguished by Hill model parameters, number of dose levels, and number of trials per dose. Fitting the Hill model to this dataset would produce estimates for $\alpha, \eta$ and $\kappa$ along with an estimated covariance matrix of the estimators of these parameters. These estimates are used to compute $90 \%$ confidence intervals for each of these three parameters. Point estimates of BMD based on extra and additional risk for BMR values of $10 \%, 5 \%$ and $1 \%$ are obtained using equations 2.20 and 2.27 , along with estimates of variance based on equations 2.25 and 2.28 and approximate $90 \%$ confidence intervals based on equation 2.26. Note that in the simulation study for a specified BMR, it is also possible to determine the true BMD value based on extra and additional risk since the true values of the Hill model parameters are known.

For each of thirty-two combinations considered here, we evaluate the performance of the estimates for the Hill model parameters $\alpha, \eta$ and $\kappa$ and of the BMD, using the relative bias (RB), relative standard deviation (RSD) and relative mean square error (RMSE) of the associated estimators computed over all 1000 simulated datasets. Generally speaking, let $\theta$ represent a parameter to be estimated $(\theta$ could be $\alpha, \eta, \kappa$ or BMD here), $\widehat{\theta}^{(r)}$ be the estimate obtained for $\theta$ from the $r^{\text {th }}$ simulated dataset $(r=1,2, \cdots, 1000)$, and $\widehat{\operatorname{Var}}^{(r)}(\widehat{\theta})$ be the associated estimate of the 
variability in $\widehat{\theta}$. Then

$$
\begin{aligned}
R B & =\left(\frac{\bar{\theta}-\theta}{\theta}\right) 100 \% \\
R S D & =\left(\frac{\sqrt{\overline{\operatorname{Var}}(\widehat{\theta})}}{\theta}\right) 100 \% \\
R M S E & =\sqrt{(R B)^{2}+(R S D)^{2}}
\end{aligned}
$$

where $\bar{\theta}=\frac{\sum_{r=1}^{1000} \widehat{\theta}^{(r)}}{1000}$ and $\overline{\operatorname{Var}}(\widehat{\theta})=\frac{\sum_{r=1}^{1000} \widehat{\operatorname{Var}}^{(r)}(\widehat{\theta})}{1000}$ In addition, using the appropriate quantities from the $r^{\text {th }}$ simulated dataset, we determine $90 \%$ large sample confidence intervals based on the standard normal distribution for $\theta$ (again, $\theta$ can be taken to be $\alpha, \eta, \kappa$ or BMD using

$$
\widehat{\theta}^{(r)} \pm Z_{0.95} \sqrt{\widehat{\operatorname{Var}}^{(r)}(\widehat{\theta})}
$$

$Z_{0.95}=1.645$ is the $95^{\text {th }}$ percentile of the standard normal percentile, and evaluate the proportion of the 1000 intervals obtained that contain the true parameter $\theta$. In what follows, we summarize the results of the simulation.

\subsection{Results and Discussion}

We present the results and discuss how the validity of normality assumption, accuracy and precision of the Hill model parameter and of the BMD estimates change with the shape of the Hill curves, number of dose levels and number of trials per dose. Normality assumption is verified using histograms obtained for these estimates. Relative bias (RB), relative standard deviation (RSD), and relative mean-squared error (RMSE) and coverage probability of the Hill model parameters - $\kappa, \eta$ and $\alpha$ - and of the benchmark dose estimates are calculated to assess the accuracy and precision of these estimates. Further, these measures are calculated for BMD estimates based on extra and additional risk for BMRs of $10 \%, 5 \%$ and $1 \%$ to identify how accuracy and precision of BMD estimates change with these factors.

Our results show that normality assumption for $\kappa$ and $\eta$ is satisfied depending on 
the value taken by $\alpha$, shape of the Hill model curves, number of dose levels and number of trials per dose. Larger the value of $\alpha$, less the normality assumption is satisfied for other two parameters as the range of probability of response decreases when $\alpha$ increases. Consequently, similarity among different values for other two parameters increases (Figures A.8 and A.9). However, that for $\alpha$ depends only on the value (0 $\rightarrow 1)$ taken by itself.
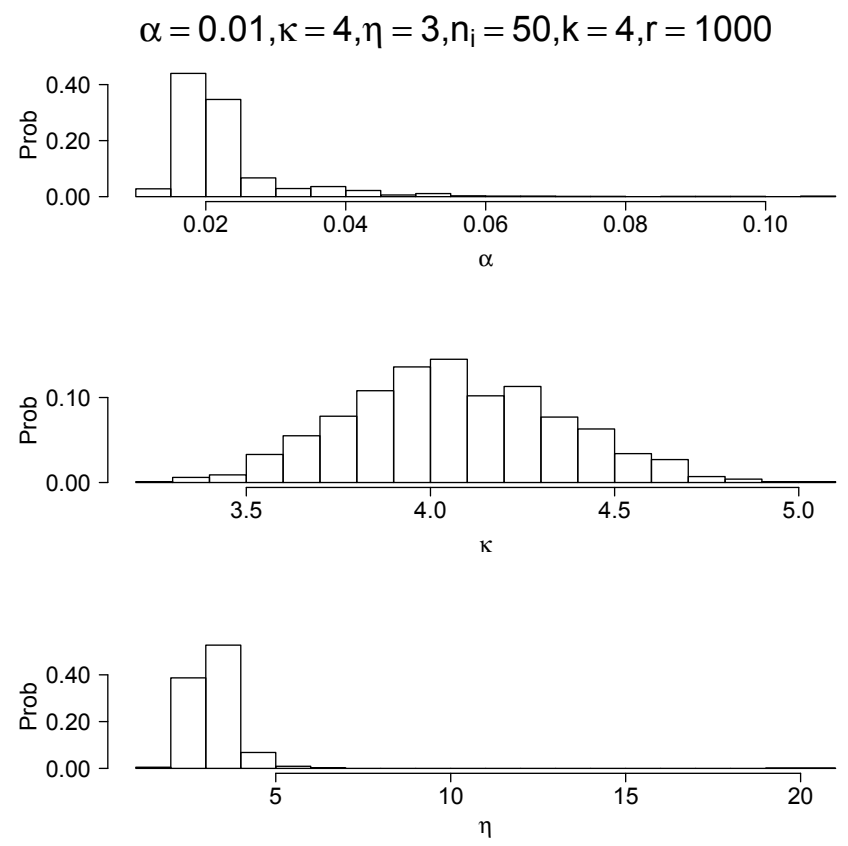

Figure 2.4: Histogram for S-shaped Hill Model Parameter Estimates when $\alpha=0.01$

Figure 2.4 presents the histograms for parameter estimates of S-shaped Hill curve with background incident of 0.01 , four dose levels and 50 trial per dose. Normality assumption for $\kappa$ and $\eta$ is better satisfied than that for $\alpha$. Figure 2.5 includes the same results when $\alpha$ is set to 0.1 . This histogram confirms that validity of normality assumption for $\alpha$ is better satisfied when $\alpha=0.1$. On S-shaped curves, effect of $\alpha$ on the validity of normality assumption of other two parameters is realized only when $\alpha$ is as high as 0.75 among the values tested which are $0.01,0.1,0.25,0.5$ and 0.75 (ref Figures 2.5 and A.10). We observe similar results for concave curve with 
initial slope greater than zero (Figure A.2) when $\alpha=0.01$ but effect of $\alpha$ on other two parameters is realized when $\alpha$ is as small as 0.5 (Figure A.12). In this histogram, we observe improvement in the normality assumption for $\alpha$.

For convex curve with background incident of 0.01, normality assumption for $\kappa$ and $\eta$ is satisfied when number of trials per dose is increased to 100 (Figure A.1). But, for concave curve with initial slope zero and $\alpha=0.01$, it is satisfied for $\kappa$ and $\eta$ when number of dose levels is increased to six (Figure A.3). For these two curves, effect of background incident on other two parameters are realized (Figures A.11 and A.13); and an improvement in the normality assumption for $\alpha$ is observed at $\alpha=0.5$.

In our research, the background incident is set to 0.01. As a result, its effect is very small in the estimates of other two parameters and consequently in the benchmark dose estimates. Therefore, we mainly focus on the characteristics of the other two parameter estimates when $\alpha$ is set to 0.01 .
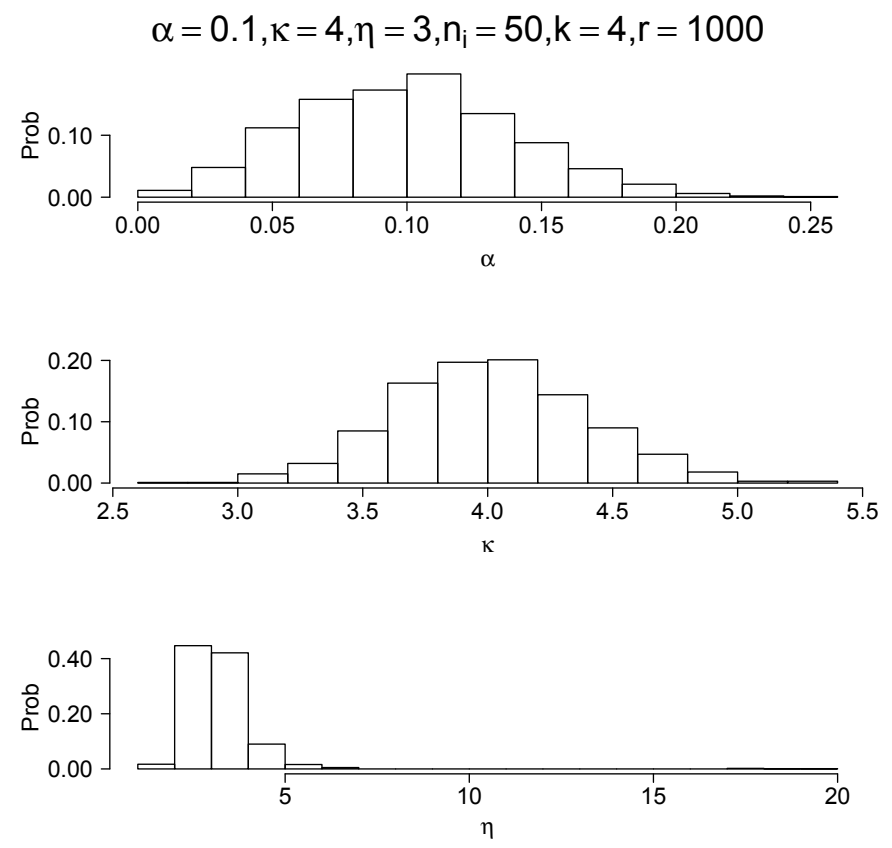

Figure 2.5: Histogram for S-shaped Hill Model Parameter Estimates when $\alpha=0.1$

Figure 2.6 of panel (a) presents the RB for the estimate of $\kappa$ for all 32 different 




(a) Relative bias of MLE of $\kappa$



(c) Relative mse of MLE of $\kappa$

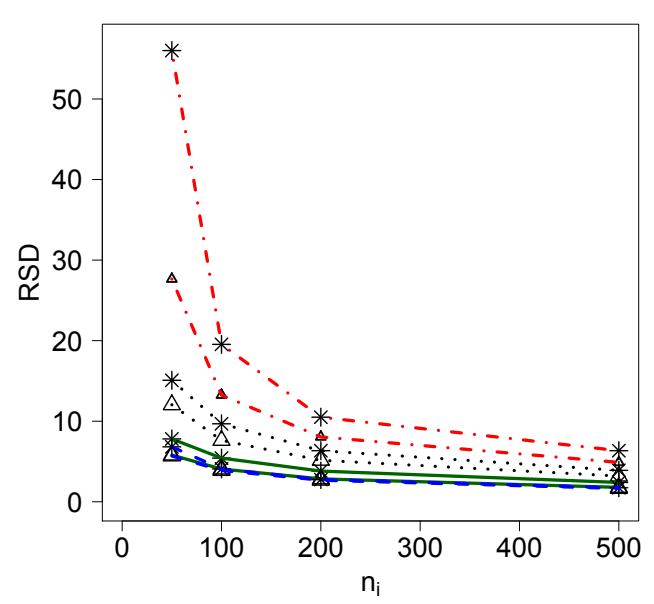

(b) Relative std of MLE of $\kappa$



(d) Confidence Coverage of $\kappa$

Figure 2.6: Variation of Properties of Estimates of $\kappa$

scenarios considered. Here, we observe the largest RB for $\kappa$ when shape of the Hill curve is convex with four doses and 50 trials per dose. Significant improvement is observed with increasing the number of trials per dose and number of dose levels, but comparatively larger improvement for the former. Effect on the RB for $\kappa$ is not as significant as convex for all the other three Hill curves while increasing the number of doses or trials per dose. Panel (b) and (c) of Figure 2.6 present the RSD and RMSE. Although overall pattern of RSD and RMSE is similar to overall pattern for RB; RSD 
and RMSE are larger for the corresponding scenarios. Figure 2.7 panel (a) presents RB for variance of MLE of $\kappa$ which is larger for convex curve when number of trials is small.

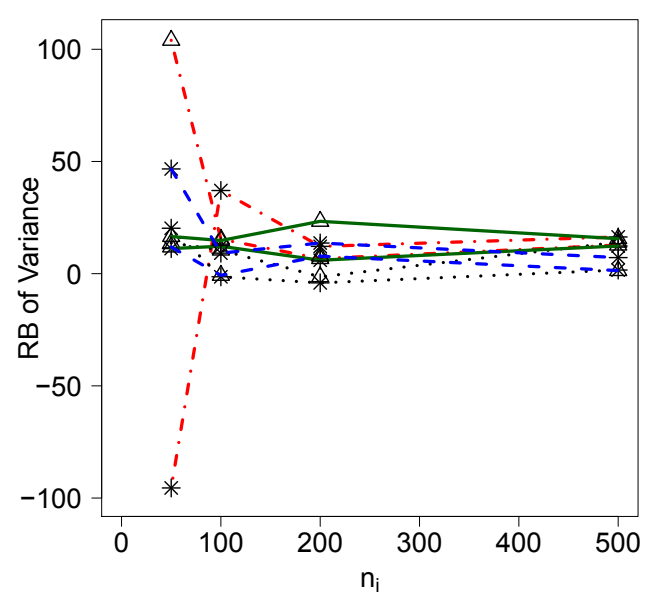

(a) $\kappa$

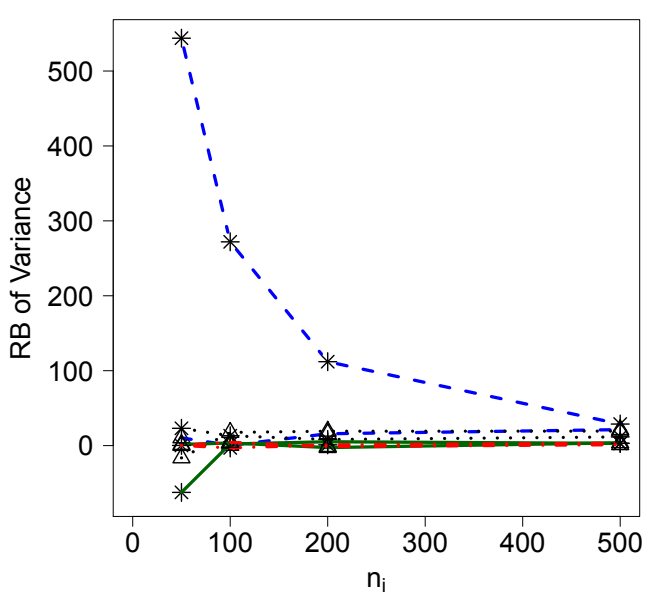

(b) $\eta$

Figure 2.7: Variation of Properties of Variance of $\kappa$ and $\eta$

The pattern that we observe for RB, RSD and RMSE may be explained by the characteristics that we observe in Figure 2.2 of panel (a). We observe that similarity among $\kappa / d_{k}$ is the highest for convex curve. Consequently, the largest of these measures are realized for this curve. The second largest RB, RSD and RMSE for $\kappa$ resulted when $\eta=2$ which has the second most homogeneity of $\kappa / d_{k}$ (Figure 2.2 in panel (b)). RB, RSD and RMSE for $\kappa$ decrease with increasing value of $\eta$ as expected, as heterogeneity of $\kappa\left(\kappa / d_{k}\right)$ increases with increasing $\eta$ (Figure 2.2 panel (a) $\rightarrow$ (d)).

Panel (d) of Figure 2.6 includes coverage probabilities with nominal band of $(0.870,0.930)$. Coverage probabilities for convex curve with smaller trials per dose do not fall within the nominal band due to having the largest RB. Further, we observe that the coverage probability is not in the nominal band for concave curve with initial slope zero with four doses while the RB, RSD and RMSE is the smallest. The reason could be that the smallest RSD may affect the coverage probability significantly even 
for smaller bias.

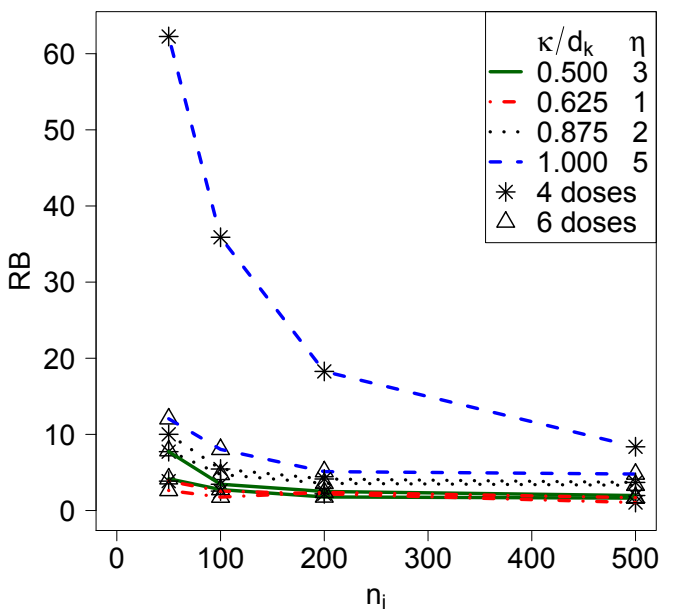

(a) Relative bias of MLE of $\eta$

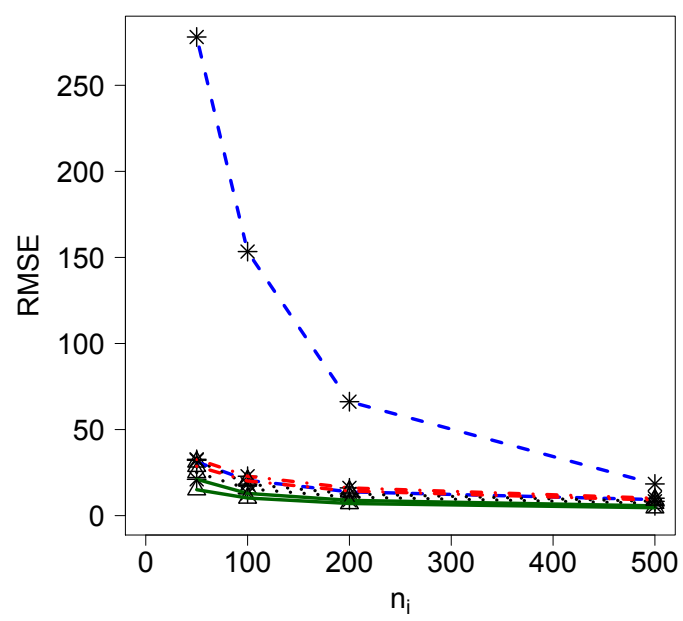

(c) Relative mse of MLE of $\eta$

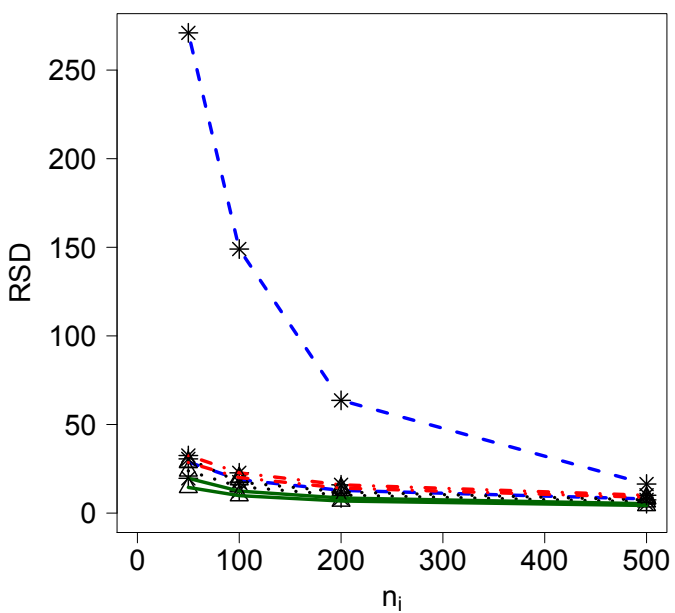

(b) Relative std of MLE of $\eta$

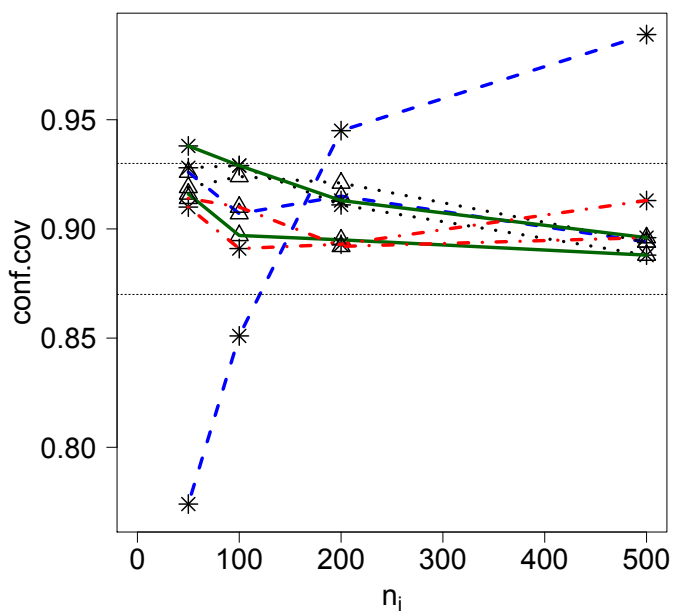

(d) Confidence Coverage of $\eta$

Figure 2.8: Variation of Properties of Estimates of $\eta$

Figure 2.8 presents the characteristics of MLEs of $\eta$. RB, RSD and RMSE for MLE of $\eta$ is the largest when $\left(\kappa / d_{k}, \eta\right)=(1.000,5)$. This characteristic could be explained by the homogeneity observed among different values of $\eta$ when $\kappa / d_{k}$ is 1.0 in panel (d) of Figure 2.3. These values significantly decrease with increasing number of dose levels than that of number of trials per dose. We observe that the 
confidence coverage of $\eta$ is out of bound when $\left(\kappa / d_{k}, \eta\right)=(1.000,5)$ with four doses but significantly improves with increasing number of dose levels than that of number of trials per dose. A similar trend is observed for relative bias of variance of $\eta$ (panel (b) of Figure 2.7).


Figure 2.9: Histogram for BMD Estimates on S-shaped Hill Curves

We briefly discuss how the value of $\alpha$ affect the estimates of other two parameters. We present RB, RSD, RMSE and coverage probability for the estimate of $\alpha$ in Table A. 4 when $\alpha$ is set to 0.01 and that in Table A.1 when $\alpha$ is set to 0.1. Comparison of these results confirms that accuracy and precision of estimates of $\alpha$ improve as the value of $\alpha$ moves closer to one as it is in the same order as the other two parameters. On the other hand, accuracy and precision of the other two parameters decreases with increasing $\alpha$. These characteristics could be explained by the increasing homogeneity observed for $\kappa$ and $\eta$ with increasing $\alpha$ (Figures A.8 and A.9). Based on these results, 
we summarize the following for the Hill model parameter estimates.

Hill model parameters are very well estimated for S-shaped curve and then for concave with initial slope greater than zero. $\eta$ is well estimated for convex curve and $\kappa$ for concave with initial slope zero. Accuracy and precision of these estimates improve with increasing number of dose levels and number trials per dose. These characteristics for $\alpha$ improves with increasing values of $\alpha$ as the value of $\alpha$ is in the same order as other two parameters. We expect that characteristics of the Hill model parameter estimates impact the estimates of the BMD and the validity of normality assumption for these estimates as they are functions of the Hill model parameter estimates.

Figure 2.9 presents the histogram for the BMD estimates for BMR values of $10 \%, 5 \%$ and $1 \%$ based on both extra and additional risk definitions, obtained for Sshaped curve with four dose levels and 50 trials per dose. We observe that normality assumption is satisfied for all six cases. Similar results are realized for concave with initial slope greater than zero (Figure A.6). For convex curve, this assumption is satisfied for BMR of $10 \%$ and 5\% (Figure A.4) with 100 trials per dose and four dose levels; but for BMR of $01 \%$ (Figure A.5) with 500 trials per dose. For concave curve with initial slope zero, this assumption is satisfied with 50 trials per dose (Figure A.7) and six dose levels instead of four. We first discuss the results that describe the characteristics of the BMD estimates for BMR of $10 \%$ based on extra risk definition and then compare how the corresponding results change when the definition of BMD and the BMR values change.

Figure 2.10 (Plots (a), (b) and (c)) presents RB, RSD and RMSE of the estimates of the benchmark dose based on extra risk with benchmark response of $10 \%$. The plots show that RB, RSD and RMSE are the largest when convex shaped Hill curve with four doses and 50 trials per dose is used. And the second largest are observed for concave with initial slope zero Hill curve with four doses and 50 number 




(a) Relative bias of $B M D 10$

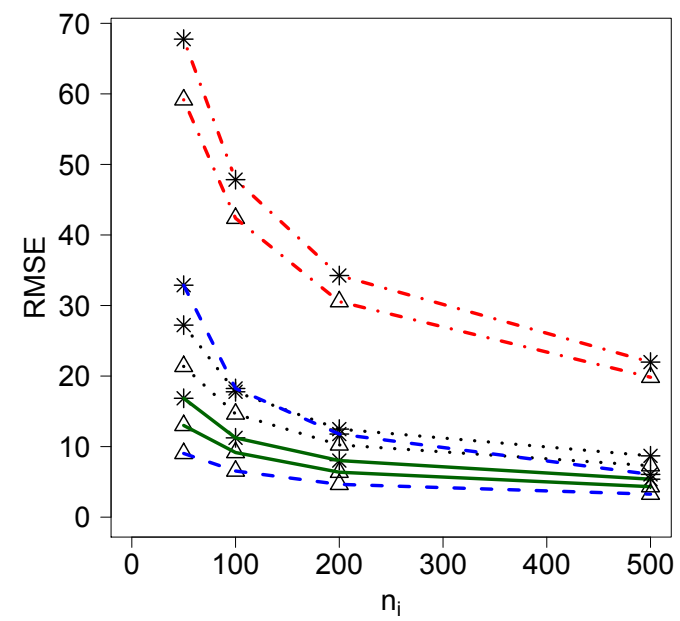

(c) Relative mse of $B M D 10$

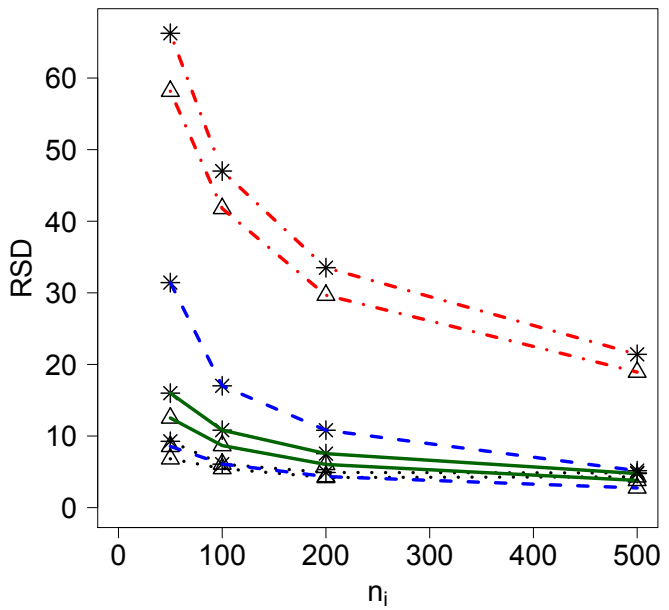

(b) Relative std of $B M D 10$

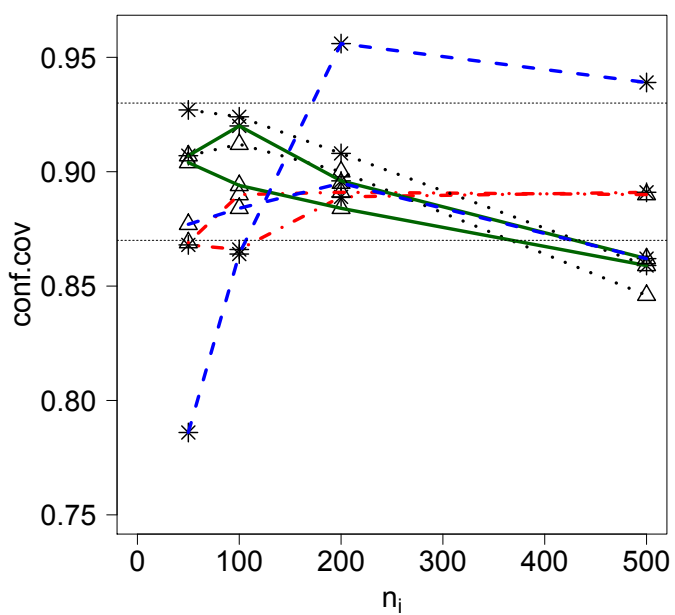

(d) Confidence Coverage of $B M D 10$

Figure 2.10: Variation of Properties of Estimates of BMD10

of trials per dose. Previously, we observe that the highest RB, RSD and RMSE for estimates of $\kappa$ is observed when convex Hill curve is used; and that of $\eta$ when concave curve with slope zero Hill curve is used. It is clear that the characteristics that is observed in the estimates of Hill model parameters are reflected in the estimates of benchmark dose which itself is a function Hill model parameter estimates. We observe similar characteristics but of increasing magnitude of RB, RSD and RMSE of 
BMD with decreasing benchmark response (Appendix A.3.4). Larger value of RB, RSD and RMSE of corresponding BMR are observed for additional risk than extra risk definition as this estimate is a function of background incident as well (Appendix A.3.5 ). These results show that characteristics observed in the estimates of Hill model parameters are reflected in the estimates of the BMD.

In addition to these observations, coverage probabilities for estimates $\kappa, \eta$ and benchmark dose decrease overall with increasing number of trials per dose and that of number of dose levels (panel (d) of Figures 2.6, $2.8,2.10$ ) because bias decreases slowly than the standard deviation for these estimates with increasing number of trials per dose and number of dose levels. Having smaller standard deviation leads to tighter confidence intervals. As a result, effect of bias on the coverage probability is higher.

RB, RSD and RMSE for variance and covariance of maximum likelihood estimates of Hill model parameters are presented in Tables A.5, A.6, A.7, A.8, A.9, A.10. Estimates of variance are in the order of $10^{-4} / 10^{-5}$ for $\alpha$; in the order of $10^{-1} / 10^{-2}$ for $\kappa$ for all except convex with four doses and 50 trials per dose; in the order of $10^{-1} / 10^{-2}$ for $\eta$ for all except concave with initial slope zero with four doses of number of trials per dose up to 200 and six dose with number of trials per dose of 50. Estimates of covariance between $\alpha$ and $\kappa$ and that between $\alpha$ and $\eta$ are very small compared to covariance between $\kappa$ and $\eta$. They are in the order of $10^{-3} / 10^{-4}$ depending on the number of trials per dose group. However, the estimates of covariance between $\kappa$ and $\eta$ are in the order of $10^{-1} / 10^{-2}$. RB, RSD and RMSE are not as high as for the other two covariances (Results in Tables A.8, A.9, A.10). We recognize a similar pattern in the size of variance and covariance of estimates of the Hill model parameters.

Similar analyzes are repeated with same number of dose levels (five), but with dose points selected at different points such as dose points at $(0,0.25,0.50,0.75,1.0)$ and $(0,0.25,0.375,0.50,1.0)$ for S-shaped curve. Results show that better estimates 
are obtained when dose points are selected at highest curvature. i.e. better results are obtained for S-shaped curve with dose points selected at $(0,0.25,0.50,0.75,1.0)$ than that at $(0,0.25,0.375,0.50,1.0)$. These results confirm the importance of having dose points at higher curvature. Additional research is needed to confirm the dose point locations.

\subsection{Summary and Conclusion}

We investigate accuracy and precision of Hill model parameter and the BMD estimates for all possible combination of four different shapes of Hill curves, two different numbers of dose levels and four different numbers of trials per dose in this chapter. These characteristics are investigated by comparing RB, RSD, RMSE and coverage probability for all thirty-two scenarios considered here. We observe that the size of these measures depend on the shape of the Hill model, the number of dose levels and the number of trials per dose. First we summarize these characteristics based on the shape of the Hill curves.

We observe that both $\kappa$ and $\eta$ are well-estimated for S-shaped Hill curve. The second best is for concave curve with initial slope greater than zero. The shape parameter $\eta$ is well-estimated for convex curve and the location parameter $\kappa$ for concave curve with initial slope zero. Accuracy and precision of these parameters are affected according to the value taken by background incident, $\alpha$ as well. Larger the value of $\alpha$, lesser the accuracy and precision of other two parameters. In our study, we fix $\alpha$ at 0.01 . Hence, effect of $\alpha$ is insignificant in the estimates of the other two parameters. We realize that these characteristics are reflected in the estimates of BMD but with different degrees depending on BMR and the benchmark definitions used. Higher the BMR, the better the BMD estimates are; and the BMD based on extra risk is better estimated than that based on additional risk. Order of the shapes of the Hill curves that gives better estimates of the BMD are: S-shaped curve, concave 
with initial slope greater than zero, concave with initial slope zero and convex. Impact of changing the number of dose levels on the accuracy and precision of these estimates varies with shape of the Hill curves.

The results show that increasing the number of dose level improves the estimates of Hill model parameters, $\kappa$ and $\eta$, and BMD estimates. This improvement is almost similar for S-shaped and concave with initial slope greater than zero. The improvement is significant in the case of concave with initial slope zero. On the other hand, it is less significant in the case convex curve. There is a noticeable improvement in the BMD estimates when number of dose levels is increased. Location of dose points are important as well. We recognize that better estimates are obtained for S-shaped curve. We compare the corresponding results with dose points selected at $(0,0.25$, $0.50,0.75,1.0)$ and that at $(0,0.25,0.375,0.50,1.0)$. Our results show that former gave better estimates. Hence, there is a need to identify the best location of dose points. In a study, Ringblom et. al. (2014) [17] studied continuous data generated by Monte Carlo simulations using four dose groups and a set of five different dose placement scenarios and pointed out the importance of model selection and sound identification of the point-of-departure in health risk assessment.

We observe a noticeable improvement in the estimates of $\kappa, \eta$ and the BMD with increasing number of trials per dose when S-shaped and concave curve with initial slope greater than zero is investigated. However, this improvement is significant when 100 trials per dose is used instead of 50 as opposed to increasing number of dose levels in the case of convex curve. In the case of concave curve with initial slope zero, improvement is significant with increasing number of trials per dose with four dose levels; and insignificant with six dose levels. Similar pattern is reflected in the estimates of BMD as it is a function of these Hill model parameter estimates, but we observe that impact of $\kappa$ is realized on the convex curve and that of $\eta$ on the concave curve with initial slope zero. 
Our studies show the situations where the estimates of BMD have higher accuracy and precision when Hill model is used to represent the dose-response relationship. Taking advantage of this background knowledge of the estimations, we assessed performance of newly introduced procedure for benchmark dose estimates.

Previously, U.S EPA [21] has provided a minimum dataset requirement for calculating BMD. Examples illustrate the cases that may fail to satisfy this minimum dataset criterion. One of them is the dataset with only the highest dose showing a response which is the hill model parameter combination $\left(\kappa / d_{k}, \eta\right)=(1,5)$. The other is dataset in which the first non-control dose has a response level substantially above the selected acceptable response which is the Hill model parameter combination $\left(\kappa / d_{k}, \eta\right)=(0.625,1)$. In such cases, U.S. EPA recommended fitting multiple models to the dataset to evaluate the magnitude of model uncertainty [21]. Our results confirm the recommendation provided by U.S. EPA.

In the literature, many dose-response curves are suggested or used. Such suggested models are as follows: linear, linear threshold, Hill, logistic, probit, log-logistic, log-probit, Weibull, gamma and linearized multistage families. It will be worth exploring whether any of the above suggested dose-response models performs better for the cases where Hill model lacks accuracy and precision so that appropriate fitted models with higher accuracy and precision can be used for already available datasets. 


\section{BIBLIOGRAPHY}

[1] Allen.B.C., Kavlock.R.J., Kimmel.C.A. and Faustman.E.M. Doseresponse assessment for developmental toxicity II. Comparison of generic benchmark dose estimates with no observed adverse effect levels, Fundamental of Applied Toxicology 1994a;23:487-495

[2] Barnes,D.G. and Dourson,M. Reference dose (RfD): description and use in health risk assessments, Regul Toxicol Pharmacol 1988;8:471-486

[3] Casarett.I.J, and Doull.J. Casarett and Doull's Toxicology: The Basic Science of Poisons, Klassssen CD (ed.), $5^{\text {th }}$ edn. McGraw-Hill: USA; 1996

[4] Crump,K. A new method for determining allowable daily intakes, Fundam Appl Toxicol 1984;4:854-871

[5] Dourson.M., Felter.S. and Robinson.D. Evolution of science-based uncertainity factors in noncancer risk assessment, Regul Toxicol Pharmacol $1996 ; 24: 108-120$

[6] EFSA. Opinion of the Scientific Committee on a request from EFSA related to a harmonised approach for risk assessment of substances which are both genotoxic and carcinogenic, The EFSA Journal 2005;282:1-31

[7] EFSA. Guidance of the Scientific Committee on a request from EFSA on the use of the benchmark dose approach in risk assessment, The EFSA Journal 2009a;1150:1-72

[8] Foster.P.M.D., and Auton.T.R. Application of benchmark dose risk assessment methodology to developmental toxicity: an industrial view, Toxicology letters 1995;82/83 555-559 
[9] Kimmell.C.A. Approaches to Evaluating Reproductive Hazards and Risks, Environ. Health Perspect. 1993;101(2), 137-143

[10] Gaylor.D.W., Ryan.L., Krewski.D., and Zhus.Y. Procedures for Calculating Benchmark Doses for Health Risk Assessment. Regulatory Toxicology and Pharmacology 1998;28:150-164

[11] Krewski. D., and Van Ryzin. J. Dose response models for quantal response toxicity data, In Statistics and Related Topics, Csorgo M, Dawson D, Rao JNK, Saleh E (eds). North-Holland: Amsterdam: 1981;201-231

[12] Lehman,A.J., and Fitzhugh,O.G. 100-fold margin of safety, Assoc.Food Drug Off. U.S.Q. Bull. 1954;18:33-35

[13] Leisenring.W and Ryan.L. Statistical Properties of the NOAEL, Regulatory Toxicology and Pharmacology 1992;15:161-171

[14] McCelellan.R.O. A historical Overview and alternative paths forward, Inhalation Toxicology 1999;11:477-518

[15] Murrell.J.A., Portier.C.J., and Morris.R.W. Characterizing DoseResponse I:Critical Assessment of the Benchmark Dose Concept, Risk Analysis Vol.18.No.1.1998

[16] Oberg.M. Benchmark dose approaches in chemical health risk assessment in relation to number and distress of laboratory animals, Regulatory Toxicology and Pharmacology 58 2010;451-454

[17] Ringblom.J., Johanson.G., and Oberg.M. Current modeling practice may lead to falsely high benchmark dose estimates, Regulatory Toxicology and Pharmacology 69 2014;171-177 
[18] Sand.S., Portier.C.J., and Krewski.D. A Signal-to-Noise Crossover Dose as the Point of Departure for Health Risk Assessment, Environ Health Perspect 2011;119:1766-1774

[19] Slikker.W.Jr., Crump.K.S., Andersen.M.E., and Bellinger.D. Biologically Based, Quantative Risk Assessment of Neurotoxicants, Fundamental and Applied Toxicology 29, 18-30 1996;Article No. 0002

[20] US EPA Guidelines for carcinogen risk assessment. Final report. EPA/630/P03/001F, Risk Assessment Forum, US EPA, Washington, DC; 2005

[21] US EPA Benchmark Dose Technical Guidance, Risk Assessment Forum, US EPA, June 2012

[22] VanLandingham.C.B., Allen.B.C., Shipp.A.M., and Crump.K.S.(2001) Comparison of the EU T25 Single Point Estimate Method with Benchmark Dose Response Modeling for Estimating Potency of Carcinogens, Risk Analysis, Vol. 21, No. 4, 2001 


\section{ESTIMATION AND ASSESSMENT OF SIGNAL-TO-NOISE CROSSOVER DOSE}

\subsection{Introduction}

Risk assessments are performed for many health effects that result from exposure to environmental agents such as radiation, other physical agents, chemicals or situations that contribute to human health or environmental hazards [23]. The major objectives of risk assessment are to assess the health risks associated with a particular type of exposure and to establish acceptable exposure levels to humans [18]. These assessments often include an analysis of dose-response relationship between exposure, and health-related outcomes so that a point departure (PoD) can be estimated.

In general, PoDs for establishing human exposure guidelines is expressed as a reference dose (RfD) [3]. The reference dose is usually determined by the formula RfD $=\mathrm{PoD} / \mathrm{UF}$, where the uncertainty factor $(\mathrm{UF})$ denotes the combined effect of inter - and intra (human variability) - species differences, the nature of the carcinogenic process and the type of reference point selected [8]. The term 'RfD' is used to represent a human exposure guideline, regardless of the type of test agent or critical endpoint considered. The value of the UF could be reduced or increased when appropriate chemical specific data are available. Essentially, the same approach is used to derive other exposure guidelines, such as tolerable daily intake (TDI), acceptable daily intake (ADI) or the margin of exposure (MOE)[8], [18]. We now discuss the various PoD measures that have been introduced in the literature.

Lehman and Fitzhugh (1954) [14] introduced the no-observed-adverse-effectlevel/lower-observed-adverse-effect-level (NOAEL/LOAEL) as a PoD for establishing human exposure guidelines. Although the NOAEL has been used for many years in the dose-response assessment, the scientific community has recognized the drawbacks 
in doing so. First, not all of the available data is used to specify the NOAEL. In fact, it has the restriction that it be equal to one of the experimental doses; hence it depends on the dose levels of the study design. If there is a wide spacing between doses, the true no adverse effect level may be considerably higher than that indicated by the experimental data. As a result, the NOAEL is not considered to be appropriate for substances that are genotoxic and carcinogenic [8].

Computer simulations and empirical studies have led to criticism of the NOAEL in terms of its sensitivity to sample size and its high sampling variability from experiment to experiment. One such study is that of Leisenring and Ryan (1992) [22]. These authors investigated the impact of the shape of the underlying dose-response curve using the Weibull models on the distribution of the NOAEL, and confirmed that the average risk level associated with the NOAEL may be substantial. They further showed that depending upon the background risk, the shape of the dose response curve and the sample size, the average risks at the NOAEL varied from 3 to $21 \%$. Because of the high variability that could be observed from experiment to experiment, unacceptable high risks could occur in using the NOAEL to obtain the RfD, even with safety factors applied.

It is well known that the NOAEL does not necessarily identify a zero risk dose below a threshold dose. Using 120 experiments reported in the literature, Gaylor (1992) [13] showed that the estimated risk of dead and reabsorbed or malformed fetuses ranged from 0 to $4.5 \%$ at the NOAEL. Since, in about one fourth of the experiments, the risk at the NOAEL exceeded 1\%, they argued that a benchmark dose with a risk in this order would eliminate higher risks and serve as a basis for establishing reference doses.

The benchmark dose (BMD) introduced by Crump (1984) [3] has initiated considerable discussion about the merits of this measure relative to the traditional NOAEL. Since the benchmark dose is chosen within or near the experimental dose 
range, it is relatively insensitive to the choice of the dose-response model, provided that an adequate fit to the experimental data is achieved. This was demonstrated for a large database of developmental toxicity (Faustman et al., 1994) [7]. Since the benchmark dose at the $1 \%$ risk level is not often precisely estimated, Auton (1994) suggested using a benchmark dose corresponding to a 5\% risk for teratology data, showing that the lower confidence limit will be comparable to the NOAEL for most datasets.

An extensive retrospective investigation of a large database for developmental toxicity demonstrates the effectiveness of the benchmark dose approach to remove some of the ambiguity in selecting a NOAEL and to avoid the wide discrepancies in risk that can occur at the NOAEL [1]. This investigation also shows that a lower confidence limit for the benchmark dose based on a $5 \%$ risk for the proportion of affected fetuses per litter tended to be centered around the NOAEL[15]. However, when no clear dose-response is obtained and/or the benchmark dose is calculated to be above the highest experimental dose level, use of the NOAEL may be more appropriate for setting an RfD (ADI). Aside from this single situation, the BMD can provide a more consistent approach that avoids the extreme behavior of the NOAEL [15] [16]. Further, Foster et. al. (1995) [11] assessed the application of the benchmark dose methodology in developmental toxicity and concluded that it has significant scientific and practical advantages over NOAEL to risk assessment.

Bokkers et. al. (2007) [4] showed that a data-based assessment factor (AF) needed for interspecies extrapolation is smaller when the point of departure is defined as a lower bound on a 95\% confidence interval on the benchmark dose (BMDL) rather than by the NOAEL, and concluded that using the default AF of 10 could lead to human exposure limits that are insufficiently protective.

In the past, the BMD method was employed primarily by regulatory authorities in the U.S., such as the U.S.Environmental Protection Agency (EPA). However, the 
European Food Safety Authority (EFSA) currently recommends the BMD approach for setting human exposure guidelines, which has increased its use in risk assessments performed by EFSA [18].

Pease et. al. (1991) [25] compared four approaches for deriving regulatory levels for reproductive toxicants by applying them to the available data on the human spermatotoxicant 1, 2-dibromo-3-chloropropane (DBCP) and showed that application of flexible uncertainty factors to the NOAEL does not provide information about the extent of health risk remaining at a regulatory level. By contrast, the benchmark dose approach provide estimates of the magnitude of sperm count reduction at a regulatory level [25].

An approach for extrapolating risk to low dose levels that takes into account the dose-response relationship as well as the lowest dose at which effects are observed, is presented by Kimmel et. al. [18]. These authors concluded that the method gives a stronger basis to estimate risk for human population, however more research is needed to develop appropriate dose-response models for the developmental toxicity data. They pointed out that improved curve-fitting of experimental data can provide better estimates for low dose extrapolation. However, determination of the acceptable level of risk and its relation to the concept of threshold needed more investigation.

Wheeler et. al. (2013) [28] performed an empirical comparison of low-dose extrapolation from PoD to extrapolations based upon methods that account for model uncertainty [28], and concluded that methods that incorporate model uncertainty provide better alternatives to linear extrapolation from a PoD. Further, they concluded that although this approach is an advancement and alternative to linear extrapolation from PoDs, more investigation is needed before a definitive recommendation can be made.

BMD and BMDL are calculated using the Delta method and bootstrap method by Zhu et. al. (2007) [22]. These methods were applied on clustered binary data 
from developmental toxicity experiments and illustrated that bootstrap BMDLs are smaller than the delta method BMDLs on average, hence quantifying risks more conservatively. The conclusion is that boostrap BMDL quantifies the uncertainty of the true BMD more honestly than the Delta method BMDL as its coverage probability is closer to nominal level than that of delta method BMDL; and BMD and BMDL estimates are generally insensitive to model choices provided that the models fit the data comparably well near the region of BMD.

Evans. et.al. (1994) [6] attempted to demonstrate a probabilistic approach to carcinogen risk assessment that employs probability trees, subjective probabilities, and standard bootstrapping procedures. However, in conclusion they acknowledged that there are several conceptual and theoretical problems with the approach. Moerbeek et. al. (2004) looked at the performance of three methods in fitting nonlinear dose-response models; the delta method, the likelihood-ratio method and the bootstrap method. They reported that the bootstrap method and likelihood-ratio method were found to give similar results; the delta method, on the other hand, resulted in narrower intervals for some cases and appeared unreliable for nonlinear dose-response models.

Kavlock et. al. (1996) [17] discussed a simulation study to assess the influence of study design on the estimation the benchmark dose. Various aspects of the study design such as the number of dose groups, dose spacing, dose placement, and size per dose group on the BMDs for two endpoints of developmental toxicity were examined. The conclusion was that the benchmark dose approach is readily applicable to the standard study designs and generally observed dose-responses, but some minor design modifications would increase the accuracy and precision of the BMD.

When using the benchmark dose as a PoD, it is felt that setting it at the $1 \%$ risk level often results in it not being precisely estimated [2]. The European Food Safety Authority (EFSA 2009a) [9] comments that the benchmark response (BMR) 
cannot be set at levels that are so low (or high) that estimation of the BMD requires extrapolation beyond the range of the experimental data, in which case the result could be highly model dependent. A new point of departure measure, the signalto-noise crossover dose, SNCD introduced by Sand et al. (2011)[18] overcomes this limitation found in BMD. The SNCD also requires model fitting just as the benchmark dose estimation does. However, this PoD differs from the BMD in that it provides an estimate of the lowest dose that can be derived for risk assessment without low-dose extrapolation.

Sand et al (2011) [18] compared the SNCD with the NOAEL and the benchmark dose as points of departure and in terms of their ability to set a reference dose. The complete NTP database was used as the basis for these analysis. The results showed that the median risk at the NOAEL was close to $10 \%$ and the default uncertainty factor $(\mathrm{UF}=100)$ was considered the most applicable to the $B M D L_{10}$. Using this observation and the default UF as a basis, a target risk of 1/1000 was used for derivation of an SNCD-based RfD by linear extrapolation. At the median, this approach provided the same RfD as the $B M D L_{10}$ divided by the default UF [18]. Sand et. al. (2011) concluded that the SNCD approach warrants further development as a PoD for human health risk assessment. For example, Sand et. al. (2011) proposed a point estimate for the signal-to-noise crossover dose. In this research, we introduce a number of methods for determining interval estimator, which we evaluate and compare via an extensive simulation study. As in chapter 2, we use the three parameter Hill model to describe the dose response relationship. In the next section, we discuss the notion underlying the signal-to-noise crossover dose.

\subsection{Signal-to-Noise Crossover Dose}

In this section, we describe the signal-to-noise crossover dose (SNCD) proposed by Sand et. al. (2011). Specifically, these authors defined the SNCD as the dose that 
produces a predetermined signal-to-noise response, SNR, which is described as ratio of additional risk to "background noise". Sand et. al. (2011) defined the background noise as the width of a $90 \%$ confidence interval for the absolute risk, $P(d)$. Thus, the SNCD is the dose, $\mathrm{d}$, which yields a predetermined signal-to-noise crossover response, SNR, according to

$$
S N R(d)=\frac{P(d)-P(0)}{P 95-P 05}
$$

The values that have been previously used for SNR to define the SNCD are 1 and 2/3. The dose, d, yielding an SNR of 1 in equation 3.1 has been referred to a SNCD1; the value of $\mathrm{d}$ giving an SNR of $2 / 3$ has been denoted by SNCD0.67. In order to develop estimates for SNCD in an empirical study, a model for $P(d)$ must be fit, estimates of P95 and P05 are also required. Once again, we use the three parameter Hill model described in 2.1 of chapter 2.2.1, which contains a background parameter, $\alpha$, a location parameter, $\kappa$, and a shape parameter, $\eta$. Maximum likelihood estimates for these parameters, along with an estimated asymptotic covariance matrix for the associated estimates are described in chapter 2.4.1.

\subsection{Estimation of Signal-to-Noise Crossover Dose}

\subsubsection{Analytical Method}

In this section, we present the development of point and interval estimates for the signal-to-noise crossover dose that are based on maximum likelihood estimates obtained for the Hill model parameters. Specifically, suppose that the Hill model in 2.1 has been fit so that the following expression can be computed at a given $d_{i}$.

$$
\widehat{P}\left(d_{i}\right)=\widehat{\alpha}+(1-\widehat{\alpha})\left(\frac{d_{i}^{\widehat{\eta}}}{\widehat{\kappa}^{\widehat{\eta}}+d_{i}^{\widehat{\eta}}}\right)
$$

The signal-to-noise crossover response can be estimated as

$$
\widehat{S N R}\left(d_{i}\right)=\frac{\widehat{P}\left(d_{i}\right)-\widehat{P}(0)}{\widehat{P 95}-\widehat{P 05}}
$$


From $3.2, \widehat{P}\left(d_{1}\right)=\widehat{\alpha}$ which yields

$$
\widehat{P}\left(d_{i}\right)-\widehat{P}(0)=(1-\widehat{\alpha})\left(\frac{d_{i}^{\widehat{\eta}}}{\widehat{\kappa}^{\widehat{\eta}}+d_{i}^{\widehat{\eta}}}\right)
$$

for the numerator of 3.3 , identifying $\widehat{P}\left(d_{i}\right)$ to be a binomial proportion allows us to write

$$
\begin{gathered}
\widehat{E}\left[\widehat{P}\left(d_{i}\right)\right]=\widehat{P}\left(d_{i}\right), \text { and } \\
\widehat{\operatorname{Var}}\left[\widehat{P}\left(d_{i}\right)\right]=\frac{\widehat{P}\left(d_{i}\right)\left(1-\widehat{P}\left(d_{i}\right)\right)}{n_{i}}
\end{gathered}
$$

Assuming normality for $\widehat{P}\left(d_{i}\right)$ (valid for values away from zero/one) allows us to approximate the denominator of $\widehat{S N R}\left(d_{i}\right)$ by the width of a $90 \%$ large sample confidence interval

$$
\widetilde{P 95}-\widetilde{P 05}=2 Z_{0.95} \sqrt{\frac{\widehat{P}\left(d_{i}\right)\left(1-\widehat{P}\left(d_{i}\right)\right)}{n_{i}}}
$$

Thus $\widehat{S N R}\left(d_{i}\right)$ in 3.3 can be approximate as

$$
\widehat{S N R}\left(d_{i}\right)=\frac{\widehat{P}\left(d_{i}\right)-\widehat{P}(0)}{2 Z_{0.95} \sqrt{\frac{\widehat{P}\left(d_{i}\right)\left(1-\widehat{P}\left(d_{i}\right)\right)}{n_{i}}}}
$$

We wish to develop a confidence interval for the SNCD at a specified level of SNR. To do so, we first consider an interval estimate for $S N R\left(d_{i}\right)$ based on 3.8. Initially, however we focus on the natural logarithm of the signal-to-noise response and the analogous estimator. Thus, taking the log of the estimate of signal-to-noise response in 3.8 at a specific dose point gives

$$
\widehat{g}\left(d_{i}\right)=\ln \widehat{S N R}\left(d_{i}\right)=\ln \left(\widehat{P}\left(d_{i}\right)-\widehat{P}(0)\right)-\ln \left[2 Z_{0.95} \sqrt{\frac{\widehat{P}\left(d_{i}\right)\left(1-\widehat{P}\left(d_{i}\right)\right)}{n_{i}}}\right]
$$

where $i=1,2, \cdots, k$. Note that $\widehat{P}\left(d_{i}\right)$ in 3.2 is a function of the Hill model parameter estimates $\widehat{\alpha}, \widehat{\kappa}$ and $\widehat{\eta}$. As such an expression for the variance of $\ln \left(\widehat{S N R}\left(d_{i}\right)\right)$ can be obtained by taking a first order multivariate Taylor Series expansion of $\ln \left(\widehat{S N R}\left(d_{i}\right)\right)$ about $\widehat{\alpha}=\alpha, \widehat{\kappa}=\kappa$ and $\widehat{\eta}=\eta$. If we define $\theta=(\alpha, \kappa, \eta)^{\prime}$ and $\widehat{\theta}=(\widehat{\alpha}, \widehat{\kappa}, \widehat{\eta})^{\prime}$, this 
expression can be written as

$\widehat{g}\left(d_{i}\right)=\ln \left(\widehat{S N R}\left(d_{i}\right)\right)=\ln \left(S N R\left(d_{i}\right)\right)+\left.\Psi_{\widehat{\alpha}}\right|_{\widehat{\theta}=\theta}(\widehat{\alpha}-\alpha)+\left.\Psi_{\widehat{\kappa}}\right|_{\widehat{\theta}=\theta}(\widehat{\kappa}-\kappa)+\left.\Psi_{\widehat{\eta}}\right|_{\widehat{\theta}=\theta}(\widehat{\eta}-\eta)$

where $\ln \left(S N R\left(d_{i}\right)\right)$ is the true unknown signal-to-noise response at specific dose level, and

$$
\begin{aligned}
& \Psi_{\widehat{\alpha}}=\left.\frac{\partial \widehat{g}\left(d_{i}\right)}{\partial \widehat{\alpha}}\right|_{\widehat{\theta}=\theta}=\frac{-1}{2}\left\{\frac{\left(\kappa^{\eta}+d_{i}^{\eta}\right)}{(1-\alpha)\left(\alpha \kappa^{\eta}+d_{i}^{\eta}\right)}\right\} \\
& \Psi_{\widehat{\kappa}}=\left.\frac{\partial \widehat{g}\left(d_{i}\right)}{\partial \widehat{\kappa}}\right|_{\widehat{\theta}=\theta}=\left(\frac{-\eta}{2 \kappa}\right)\left\{\frac{\alpha \kappa^{\eta}}{\left(\alpha \kappa^{\eta}+d_{i}^{\eta}\right)}+1\right\} \\
& \Psi_{\widehat{\eta}}=\left.\frac{\partial \widehat{g}\left(d_{i}\right)}{\partial \widehat{\eta}}\right|_{\widehat{\theta}=\theta}=\frac{\left(\ln \left(d_{i}\right)-\ln (\kappa)\right)\left(2 \alpha \kappa^{\eta}+d_{i}^{\eta}\right)}{2\left(\alpha \kappa^{\eta}+d_{i}^{\eta}\right)}
\end{aligned}
$$

Taking the variance of 3.10 allows us to write that

$$
\begin{aligned}
\operatorname{Var}\left(\ln \widehat{S N R}\left(d_{i}\right)\right) \approx & \Psi_{\alpha}^{2} \operatorname{Var}(\widehat{\alpha})+\Psi_{\kappa}^{2} \operatorname{Var}(\widehat{\kappa})+\Psi_{\eta}^{2} \operatorname{Var}(\widehat{\eta})+2 \Psi_{\alpha} \Psi_{\kappa} \operatorname{Cov}(\widehat{\alpha}, \widehat{\kappa}) \\
& +2 \Psi_{\alpha} \Psi_{\eta} \operatorname{Cov}(\widehat{\alpha}, \widehat{\eta})+2 \Psi_{\kappa} \Psi_{\eta} \operatorname{Cov}(\widehat{\kappa}, \widehat{\eta})=\sigma_{g}^{2}
\end{aligned}
$$

which can be estimated by

$$
\begin{aligned}
\widehat{\operatorname{Var}}\left(\ln \widehat{\operatorname{SNR}}\left(d_{i}\right)\right) \approx & \widehat{\Psi}_{\alpha}^{2} \widehat{\operatorname{Var}}(\widehat{\alpha})+\widehat{\Psi}_{\kappa}^{2} \widehat{\operatorname{Var}}(\widehat{\kappa})+\widehat{\Psi}_{\eta}^{2} \widehat{\operatorname{Var}}(\widehat{\eta})+2 \widehat{\Psi}_{\alpha} \widehat{\Psi}_{\kappa} \widehat{\operatorname{Cov}}(\widehat{\alpha}, \widehat{\kappa}) \\
& +2 \widehat{\Psi}_{\alpha} \widehat{\Psi}_{\eta} \widehat{\operatorname{Cov}}(\widehat{\alpha}, \widehat{\eta})+2 \widehat{\Psi}_{\kappa} \widehat{\Psi}_{\eta} \widehat{\operatorname{Cov}}(\widehat{\kappa}, \widehat{\eta})=\widehat{\sigma}_{g}^{2}
\end{aligned}
$$

where the estimated variances and covariances of $\widehat{\alpha}, \widehat{\kappa}$ and $\widehat{\eta}$ are available from the estimated asymptotic covariance matrix of the estimates of the Hill model parameters, and $\widehat{\Psi}_{\widehat{\alpha}}, \widehat{\Psi}_{\widehat{\kappa}}$ and $\widehat{\Psi}_{\widehat{\eta}}$ are computed by replacing $\alpha, \kappa$ and $\eta$ in $\Psi_{\widehat{\alpha}}, \Psi_{\widehat{\kappa}}$ and $\Psi_{\widehat{\eta}}$ with $\widehat{\alpha}$, $\widehat{\kappa}$ and $\widehat{\eta}$. An expression for the expectation of $\ln \left(\widehat{S N R}\left(d_{i}\right)\right)$ can be obtained by taking a second order multivariate Taylor Series expansion of $\ln \left(\widehat{S N R}\left(d_{i}\right)\right)$ about $\widehat{\alpha}=\alpha$, $\widehat{\kappa}=\kappa$ and $\widehat{\eta}=\eta$. This expression is 


$$
\begin{aligned}
& \widehat{g}\left(d_{i}\right)=\ln \left(\widehat{S N R}\left(d_{i}\right)\right) \approx \ln \left(S N R\left(d_{i}\right)\right)+\left.\Psi_{\widehat{\alpha}}\right|_{\widehat{\theta}=\theta}(\widehat{\alpha}-\alpha)+\left.\Psi_{\widehat{\kappa}}\right|_{\widehat{\theta}=\theta}(\widehat{\kappa}-\kappa) \\
& +\left.\Psi_{\widehat{\eta}}\right|_{\widehat{\theta}=\theta}(\widehat{\eta}-\eta)+\left.\Psi_{\widehat{\alpha} \widehat{\alpha}}\right|_{\widehat{\theta}=\theta} \frac{(\widehat{\alpha}-\alpha)^{2}}{2 !}+\left.\Psi_{\widehat{\kappa} \widehat{\kappa}}\right|_{\widehat{\theta}=\theta} \frac{(\widehat{\kappa}-\kappa)^{2}}{2 !}+\Psi_{\left.\widehat{\eta} \widehat{\eta}\right|_{\widehat{\theta}=\theta}} \frac{(\widehat{\eta}-\eta)^{2}}{2 !} \\
& +\left.\Psi_{\widehat{\alpha} \widehat{\kappa}}\right|_{\widehat{\theta}=\theta} \frac{(\widehat{\alpha}-\alpha)(\widehat{\kappa}-\kappa)}{2 !}+\left.\Psi_{\widehat{\alpha} \widehat{\eta}}\right|_{\widehat{\theta}=\theta} \frac{(\widehat{\alpha}-\alpha)(\widehat{\eta}-\eta)}{2 !}+\left.\Psi_{\widehat{\kappa} \widehat{\eta}}\right|_{\widehat{\theta}=\theta} \frac{(\widehat{\kappa}-\kappa)(\widehat{\eta}-\eta)}{2 !}
\end{aligned}
$$

where $\ln \left(S N R\left(d_{i}\right)\right)$ is once again the true unknown signal-to-noise response, $\Psi_{\widehat{\alpha}}, \Psi_{\widehat{\kappa}}$ and $\Psi_{\widehat{\eta}}$ are in $3.11,3.12$ and 3.13 ; and

$$
\begin{aligned}
& \Psi_{\widehat{\alpha} \widehat{\alpha}}=\left.\frac{\partial^{2} \widehat{g}\left(d_{i}\right)}{\partial \widehat{\alpha}^{2}}\right|_{\widehat{\theta}=\theta}=\frac{\kappa^{2 \eta}}{2\left(\alpha \kappa^{\eta}+d_{i}^{\eta}\right)^{2}}-\frac{1}{2(1-\alpha)^{2}} \\
& \Psi_{\widehat{\kappa} \widehat{\kappa}}=\left.\frac{\partial^{2} \widehat{g}\left(d_{i}\right)}{\partial \widehat{\kappa}^{2}}\right|_{\widehat{\theta}=\theta}=\frac{\eta}{2 \kappa}\left\{1+\alpha \kappa^{(\eta-1)}\left(\frac{\alpha \kappa^{\eta}-(\eta-1) d_{i}^{\eta}}{\left(\alpha \kappa^{\eta}+d_{i}^{\eta}\right)^{2}}\right)\right\} \\
& \Psi_{\widehat{\eta} \widehat{\eta}}=\left.\frac{\partial^{2} \widehat{g}\left(d_{i}\right)}{\partial \widehat{\eta}^{2}}\right|_{\widehat{\theta}=\theta}=\frac{\alpha \kappa^{\eta} d_{i}^{\eta}\left(\ln (\kappa)-\ln \left(d_{i}\right)\right)}{\left(\alpha \kappa^{\eta}+d_{i}^{\eta}\right)^{2}} \\
& \Psi_{\widehat{\alpha} \widehat{\kappa}}=\left.\frac{\partial^{2} \widehat{g}\left(d_{i}\right)}{\partial \widehat{\alpha} \partial \widehat{\kappa}}\right|_{\widehat{\theta}=\theta}=\frac{-\eta \kappa^{(\eta-1)} d_{i}^{\eta}}{2\left(\alpha \kappa^{\eta}+d_{i}^{\eta}\right)^{2}} \\
& \Psi_{\widehat{\alpha} \widehat{\eta}}=\left.\frac{\partial^{2} \widehat{g}\left(d_{i}\right)}{\partial \widehat{\alpha} \partial \widehat{\eta}}\right|_{\widehat{\theta}=\theta}=\frac{\kappa^{\eta} d_{i}^{\eta}\left(\ln \left(d_{i}\right)-\ln (\kappa)\right)}{2\left(\alpha \kappa^{\eta}+d_{i}^{\eta}\right)^{2}} \\
& \Psi_{\widehat{\kappa} \widehat{\eta}}=\left.\frac{\partial^{2} \widehat{g}\left(d_{i}\right)}{\partial \widehat{\kappa} \partial \widehat{\eta}}\right|_{\widehat{\theta}=\theta}=\frac{1}{2 \kappa}\left\{\alpha \kappa^{\eta}\left(\frac{\left(\alpha \kappa^{\eta}+d_{i}^{\eta}\right)+\eta d_{i}^{\eta}\left(\ln (\kappa)-\ln \left(d_{i}\right)\right)}{\left(\alpha \kappa^{\eta}+d_{i}^{\eta}\right)^{2}}\right)-1\right\}
\end{aligned}
$$

Taking the expectation of 3.16 gives

$$
\begin{aligned}
E\left(\widehat{g}\left(d_{i}\right)\right)= & E\left(\ln \widehat{\operatorname{SNR}}\left(d_{i}\right)\right) \approx \ln \left(\operatorname{SNR}\left(d_{i}\right)\right) \\
& +(1 / 2) \Psi_{\alpha \alpha} \operatorname{Var}(\widehat{\alpha})+(1 / 2) \Psi_{\kappa \kappa} \operatorname{Var}(\widehat{\kappa})+(1 / 2) \Psi_{\eta \eta} \operatorname{Var}(\widehat{\eta}) \\
& +(1 / 2) \Psi_{\alpha \kappa} \operatorname{Cov}(\widehat{\alpha}, \widehat{\kappa})+(1 / 2) \Psi_{\alpha \eta} \operatorname{Cov}(\widehat{\alpha}, \widehat{\eta})+(1 / 2) \Psi_{\kappa \eta} \operatorname{Cov}(\widehat{\kappa}, \widehat{\eta})=\mu_{g}
\end{aligned}
$$

which can be estimated by

$$
\begin{aligned}
\widehat{E}\left(\widehat{g}\left(d_{i}\right)\right)= & \widehat{E}\left(\ln \widehat{\operatorname{SNR}}\left(d_{i}\right)\right) \approx \ln \left(\widehat{\operatorname{SNR}}\left(d_{i}\right)\right) \\
& +(1 / 2) \widehat{\Psi}_{\alpha \alpha} \widehat{\operatorname{Var}}(\widehat{\alpha})+(1 / 2) \widehat{\Psi}_{\kappa \kappa} \widehat{\operatorname{Var}}(\widehat{\kappa})+(1 / 2) \widehat{\Psi}_{\eta \eta} \widehat{\operatorname{Var}}(\widehat{\eta}) \\
& +(1 / 2) \widehat{\Psi}_{\alpha \kappa} \widehat{\operatorname{Cov}}(\widehat{\alpha}, \widehat{\kappa})+(1 / 2) \widehat{\Psi}_{\alpha \eta} \widehat{\operatorname{Cov}}(\widehat{\alpha}, \widehat{\eta})+(1 / 2) \widehat{\Psi}_{\kappa \eta} \widehat{\operatorname{Cov}}(\widehat{\kappa}, \widehat{\eta})=\widehat{\mu}_{g}
\end{aligned}
$$


If we choose to ignore the bias in $\ln \left(\widehat{S N R}\left(d_{i}\right)\right)$, an approximate $90 \%$ confidence interval for $\ln \left(S N R\left(d_{i}\right)\right)$ for $i=1,2, \cdots, k$ is

$$
\ln \left(\widehat{S N R}\left(d_{i}\right)\right) \pm Z_{0.95} \sqrt{\widehat{\operatorname{Var}}\left(\ln \left(\widehat{\operatorname{SNR}}\left(d_{i}\right)\right)\right)}
$$

We can estimate an approximate $90 \%$ confidence interval for $S N R\left(d_{i}\right)$ by exponentiating the upper and lower limits of the interval in 3.25 .

$$
\begin{aligned}
& \widehat{S N R} R_{95}\left(d_{i}\right)=\exp \left(\ln \left(\widehat{S N R}\left(d_{i}\right)\right)+Z_{0.95} \sqrt{\widehat{\operatorname{Var}}\left(\ln \left(\widehat{S N R}\left(d_{i}\right)\right)\right)}\right) \\
& \widehat{S N R} R_{05}\left(d_{i}\right)=\exp \left(\ln \left(\widehat{S N R}\left(d_{i}\right)\right)-Z_{0.95} \sqrt{\widehat{\operatorname{Var}}\left(\ln \left(\widehat{S N R}\left(d_{i}\right)\right)\right)}\right)
\end{aligned}
$$

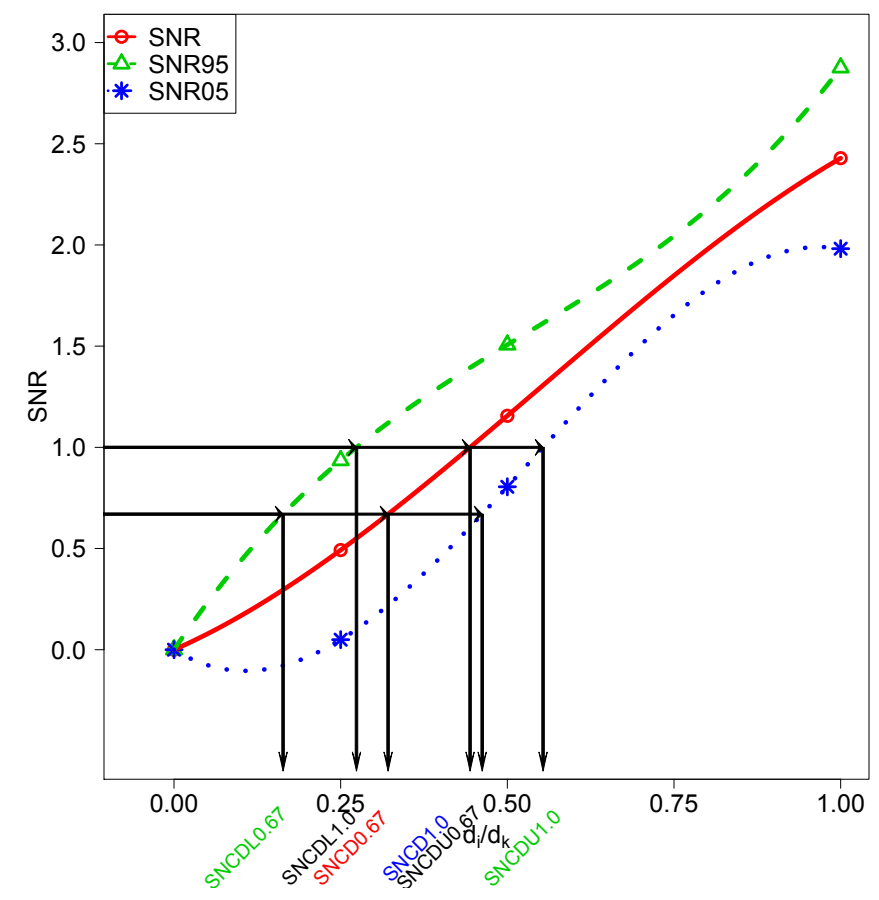

Figure 3.1: Graphical Display of SNCDL, SNCD and SNCDU

Equation 3.26 can be used to determine lower and upper $90 \%$ confidence interval bounds, $\widehat{S N R} R_{05}\left(d_{i}\right)$ and $\widehat{S N R}{ }_{95}\left(d_{i}\right)$ for each $d_{i}=1,2, \cdots, k$ specified in a given experimental study. Values of point estimates for SNR, $\widehat{S N R}\left(d_{i}\right)$, are also available for all $i=1,2, \cdots, k$. For each of $\left(d_{i}, \widehat{S N R}\left(d_{i}\right)\right),\left(d_{i}, \widehat{S N R} R_{05}\left(d_{i}\right)\right)$, and $\left(d_{i}, \widehat{S N R} R_{95}\left(d_{i}\right)\right)$ 
, $i=1,2, \cdots, k$, we fit dose versus signal-to-noise response curves using spline interpolation; an example of the resulting plot is presented in Figure 3.1, where the horizontal axis is defined as $d_{i} / d_{k}$, where $d_{k}$ is the maximum dose used in the study. Recall that the signal-to-noise crossover dose is defined as the dose, d, required to achieve a specified signal-to-noise response. Two common choices for the signalto-noise response are 1 and $2 / 3$. To determine a $90 \%$ confidence interval for $S N C D 1$, the signal-to-noise crossover dose for an SNR of one, we can simply read horizontally across in Figure 3.1 at a value of one on the vertical axis, and specify the interval as the dose levels corresponding to where $\widehat{S N R} \widehat{S 5}_{05}$ and $\widehat{S N R} \widehat{S 5}_{95}$ are equal to one. The point estimate of $S N C D 1$ is the dose corresponding to $\widehat{S N R}=1$; that of $S N C D L 1$ is the dose corresponding to $\widehat{S N R} \widehat{S F}_{95}=1$; and $S N C D U 1$ is that corresponding to $\widehat{S N R} R_{05}=1$. We can obtain analogous point and interval estimates for the case where the SNR is $2 / 3$; these are displayed as $S N C D 0.67, S N C D L 0.67$ and $S N C D U 0.67$ in Figure 3.1.

An alternative approach for developing an interval would involve bootstrapping. On a single dataset with Bernoulli outcomes with number trials of $\left(n_{1}, n_{2}, \cdots, n_{k}\right)$ at dose levels $\left(d_{1}, d_{2}, \cdots, d_{k}\right)$, we sampled with replacement with size of $\left(n_{1}, n_{2}, \cdots, n_{k}\right)$ at dose levels $\left(d_{1}, d_{2}, \cdots, d_{k}\right)$. Number of successes, $\left(x_{b 1}, x_{b 2}, \cdots, x_{b k}\right)$, for these Bernoulli outcomes are obtained at each dose levels. We fitted the Hill model in 2.1 on this bootstrapped dataset. Maximum likelihood and variance-covariance estimates of Hill model parameters, $\alpha, \kappa$ and $\eta$ are obtained as described in chapter 2. By repeating the analytical approach described in chapter 3.3.1, we derived the $\widehat{S N R}\left(d_{i}\right)$ at each dose points for $i=1,2, \cdots, k$ and consequently obtained the estimate of SNCD of $\mathrm{p}=1$ and $2 / 3$ for this bootstrapped dataset. We repeated this bootstrapping 500 times and obtained 500 estimates of SNCD. Mean and variance of these SNCD estimates are

$$
\overline{S N C D}=\frac{\sum_{b=1}^{500} S \widehat{N C} D^{(b)}}{500}
$$




$$
\widehat{\operatorname{Var}}(S \widehat{N C} D)=\frac{\sum_{b=1}^{500}\left(S \widehat{N C} D^{(b)}-\overline{S N C D}\right)^{2}}{499}
$$

Under normality assumption for $S N C D$, upper $\left(S N C D_{95}\right)$ and lower $\left(S N C D_{05}\right) 90 \%$ large sample confidence interval for the estimate of SNCD is given by,

$$
S \widehat{N C} D \pm Z_{0.95} \sqrt{\widehat{\operatorname{Var}}(S \widehat{N C} D)}
$$

\subsubsection{Bootstrap Method}

In addition to a confidence interval for SNCD using the analytic approach, we also propose two methods that are based on bootstrap techniques. We discuss these in the context of a simulation study that we present in the chapter 3.4.2.

\subsection{Simulation Study}

To assess the inference procedures proposed here for SNCD that are based on a three parameter Hill model, a simulation study is conducted. In chapter 2, we presente the conditions under which the Hill model parameters are well-estimated as there would be repercussions on the point and interval estimates of SNCD when this would not be the case. We investigate thirty-two different scenarios based doseresponse curve, number of dose levels, and number of trials as presented in chapter 2.

We simulate 1000 datasets for each of the different scenarios that are distinguished by dose response relationship, number of dose levels, number of trials per dose and maximum dose. However, here we includ the number of dose levels of eight $\left(d_{i} / d_{k}=0,0.25,0.375,0.5,0.625,0.75,0.875,1.0\right)$ in addition to four and six; and add the number of trials at each dose levels to be either $n_{i}=300$ or 400 in addition to what is used in chapter 2 . 


\subsubsection{Analytical Method}

We estimate $S N C D L, S N C D, S N C D U, S N C D_{05}$, and $S N C D_{95}$ for SNR of both 1 and 2/3 for each of the 1000 simulated datasets from each of the seventytwo different scenarios mentioned above. Coverage probability based on (SNCDL, SNCDU) is given by

$$
c P b_{L U}=\frac{\sum_{r=1}^{1000} \mathbb{1}_{L U}^{(r)}}{1000}
$$

where

$$
\mathbb{1}_{L U}^{(r)}= \begin{cases}1, & \text { if } S N C D L \leq S N C D_{t r} \leq S N C D U \\ 0, & \text { otherwise }\end{cases}
$$

Coverage probability based on $\left(S N C D_{05}, S N C D_{95}\right)$ is given by

$$
c P b_{0595}=\frac{\sum_{r=1}^{1000} \mathbb{1}_{0595}^{(r)}}{1000}
$$

where

$$
\mathbb{1}_{0595}^{(r)}= \begin{cases}1, & \text { if } S N C D_{05} \leq S N C D_{t r} \leq S N C D_{95} \\ 0, & \text { otherwise }\end{cases}
$$

Note that it is possible to estimate the true SNCD of a specified SNR since the true values of Hill model parameters are known here. This allows us to estimate the true bias of SNCD which is given by,

$$
\text { Bias }_{S N C D}=\frac{\sum_{r=1}^{1000} S N C D^{(r)}}{1000}-S N C D_{\text {true }}
$$

As this realized bias of SNCD turn out to be significant, bias-correction was performed on these two different confidence intervals. Bias corrected coverage probability $\left(C c P b_{L U}\right)$ based on (SNCDL, SNCDU) is estimated as given in 3.30, but with

$$
\mathbb{1}_{L U}^{(r)}= \begin{cases}1, & \text { if }\left(S N C D L-\operatorname{Bias}_{S N C D}\right) \leq S N C D_{t r} \leq\left(S N C D U-\text { Bias }_{S N C D}\right) \\ 0, & \text { otherwise }\end{cases}
$$


Similarly bias corrected coverage probability $\left(C c P b_{0595}\right)$ based on $\left(S N C D_{05}, S N C D_{95}\right)$ is as given in 3.32 , but with

$$
\mathbb{1}_{0595}^{(r)}= \begin{cases}1, & \text { if }\left(S N C D_{05}-\text { Bias }_{S N C D}\right) \leq S N C D_{t r} \leq\left(S N C D_{95}-\text { Bias }_{S N C D}\right) \\ 0, & \text { otherwise }\end{cases}
$$

We calculate this bias, two different uncorrected coverage probabilities and two different bias-corrected coverage probabilities for signal-to-noise response of 1 and $2 / 3$. These estimations are carried out for all 72 different scenarios based on four dose-response curves, three different numbers of dose levels and six different numbers of trials per dose. In addition to estimating these measures using the analytical approach, parametric and non-parametric bootstrap approaches are applied as well.

\subsubsection{Bootstrap Method}

For a single dataset with number of trials, $\left(n_{1}, n_{2}, \cdots, n_{k}\right)$, and number of successes of $\left(x_{1}, x_{2}, \cdots, x_{k}\right)$ at the dose level of $\left(d_{1}, d_{2}, \cdots, d_{k}\right)$, we applied bootstrap method in two different ways depending on whether parametric or non-parametric bootstrap method is used. We first present the parametric bootstrap approach.

\subsubsection{Parametric Bootstrap Method}

In this method, Hill model, given in 2.1, is fitted to the above described dataset. Response, $\widehat{P}\left(d_{i}\right)$ in 3.2 , is estimated by substituting the maximum likelihood estimates of the fitted Hill model parameters, $\alpha, \kappa$ and $\eta$ at each dose points.

Bootstrap sample B1 of Bernoulli outcomes of size $\left(n_{1}, \cdots, n_{k}\right)$ at dose points $\left(d_{1}, d_{1}, \cdots, d_{k}\right)$ are simulated using the estimated response, $\left(\widehat{P}\left(d_{1}\right), \cdots, \widehat{P}\left(d_{k}\right)\right)$. Number of successes $\left(x_{1}^{(1)}, \cdots, x_{k}^{(1)}\right)$ at each dose points are obtained from these Bernoulli outcomes. Now, we have the responses of $\left(x_{1}^{(1)} / n_{1}, x_{1}^{(1)} / n_{1}, \cdots, x_{k}^{(1)} / n_{k}\right)$ at dose levels $\left(d_{1}, d_{1}, \cdots, d_{k}\right)$. Upper and lower bound of $90 \%$ binomial profile likelihood confidence 
interval is calculated for responses, $\left(x_{1}^{(1)} / n_{1}, x_{1}^{(1)} / n_{1}, \cdots, x_{k}^{(1)} / n_{k}\right)$. The corresponding $\left(S N R^{(1)}\left(d_{1}\right), \cdots, S N R^{(1)}\left(d_{k}\right)\right)$ are calculated using Equation 3.3 for the bootstrap sample B1. Using spline interpolation of $d_{i}$ versus $\widehat{S N R}\left(d_{i}\right)$ for $i=1,2, \cdots, k$, SNCD (of $\mathrm{p}=1$ and 2/3) is estimated as shown in Figure 3.1 and following the associated steps involved in that section (page 47).

For bootstrap sample B1 of Bernoulli outcomes with number of trials of $\left(n_{1}, n_{2}\right.$, $\left.\cdots, n_{k}\right)$ at dose points $\left(d_{1}, d_{2}, \cdots, d_{k}\right)$, we carry out sampling with replacement of size $\left(n_{1}, n_{2}, \cdots, n_{k}\right)$. The steps described above are repeated for this sample to estimate the associated SNCD (of $\mathrm{p}=1$ and 2/3). This sampling with replacement procedure is repeated 500 times. Consequently, 500 estimates of $\left(S N R^{(1)}\left(d_{1}\right), \cdots, S N R^{(1)}\left(d_{k}\right)\right)$ at each dose point and 500 estimates of SNCD are calculated. Hence, variance of $\widehat{S N R}\left(d_{i}\right)$ for one bootstrap sample, say B1, is

$$
\widehat{\operatorname{Var}}\left(\widehat{S N R}\left(d_{i}\right)\right)=\frac{\sum_{b=1}^{500}\left(\widehat{S N R}^{(b)}\left(d_{i}\right)-\overline{S N R}\left(d_{i}\right)\right)^{2}}{499}
$$

where

$$
\overline{S N R}\left(d_{i}\right)=\frac{\sum_{b=1}^{500} \widehat{S N R}^{(b)}\left(d_{i}\right)}{500}
$$

for $i=1,2, \cdots, k$.

For this bootstrap sample, upper $\left.\widehat{(S N R}\left(d_{i}\right) 95\right)$ and lower $\left(\widehat{S N R}\left(d_{i}\right) 05\right)$ bound of $90 \%$ confidence interval for $\widehat{S N R}\left(d_{i}\right)$ is

$$
\widehat{S N R}\left(d_{i}\right) \pm Z_{0.95} \sqrt{\widehat{\operatorname{Var}}\left(\widehat{S N R}\left(d_{i}\right)\right)}
$$

Using spline interpolation of $\left(d_{i}\right.$ versus $\left.\widehat{S N R} R_{95}\left(d_{i}\right)\right),\left(d_{i}\right.$ versus $\left.\widehat{S N R}\left(d_{i}\right)\right)$ and $\left(d_{i}\right.$ versus $\left.\widehat{S N R} R_{05}\left(d_{i}\right)\right)$ for $\mathrm{i}=1,2, \cdots, \mathrm{k}$, estimates of $S N C D L, S N C D$ and $S N C D U$ for a specific value of SNR (1 or 2/3) is calculated by repeating the associated steps involved in Figure 3.1 for the bootstrap sample.

Variance of $S \widehat{N C} D$ can be estimated from the 500 SNCD estimates using

$$
\widehat{\operatorname{Var}}(\widehat{S N C} D)=\frac{\sum_{b=1}^{500}\left(S \widehat{N C} D^{(b)}-\overline{S N C D}\right)^{2}}{499}
$$


where

$$
\overline{S N C D}=\frac{\sum_{b=1}^{500} S \widehat{N C} D^{(b)}}{500}
$$

For bootstrap sample B1, upper $\left(S \widehat{N C} D_{95}\right)$ and lower $\left(S \widehat{N C} D_{05}\right)$ bound of $90 \%$ confidence interval for $S \widehat{N C} D$ is

$$
S \widehat{N C} D \pm Z_{0.95} \sqrt{\widehat{\operatorname{Var}}(S \widehat{N C} D)}
$$

These steps are repeated for another 999 bootstrap samples of $B 2, \cdots, B 1000$. Now, we have 1000 estimates of $S N C D L, S N C D, S N C D U, S N C D_{05}$ and $S N C D_{95}$. Note again that it is possible to estimate the true SNCD of specified SNR using the fitted Hill model parameter estimates. This allows us to estimate the true bias of SNCD using 3.34. Uncorrected coverage probability based on (SNCDL, SNCDU) using 3.30 and 3.31 and bias corrected using 3.35; that of uncorrected based on $\left(S N C D_{05}, S N C D_{95}\right)$ using 3.32 and 3.33 and bias corrected using 3.36 are calculated.

\subsubsection{Non-parametric Bootstrap Method}

From a single dataset with number of successes of $\left(x_{1}, x_{2}, \cdots, x_{k}\right)$ with number of trials of $\left(n_{1}, \cdots, n_{k}\right)$, non-parametric bootstrap sample B1 with Bernoulli outcomes of size $\left(n_{1}, \cdots, n_{k}\right)$ is created using response of $\left(x_{1} / n_{1}, \cdots, x_{k} / n_{k}\right)$. For this non-parametric bootstrap sample, the steps followed in the parametric bootstrap technique are followed and similar results are obtained.

\subsection{Results and Discussion}

\subsubsection{Estimation of Signal-to-Noise Crossover Dose}

We discuss the details of how the true bias, uncorrected and bias-corrected coverage probability based on (SNCDL, SNCDU) and $\left(S N C D_{05}, S N C D_{95}\right)$ change depending on the shape of the Hill model curve, number of dose levels and number of 
trials per dose when applying analytical, parametric and non-parametric bootstrap methods for 72 different scenarios.

\subsubsection{Analytical Method}

We present the results obtained using the analytical approach here. Bias, uncorrected and bias corrected coverage probability based on (SNCDL, SNCDU) and $\left(S N C D_{05}, S N C D_{95}\right)$ of the four hill model parameter combinations of $(\kappa / \mathrm{mxd}, \eta)=$ $((0.500,3),(0.625,1),(0.875,2),(1,5))$ with four, six and eight doses; and 50, 100, 200, 300, 400 and 500 trials per dose are investigated here.

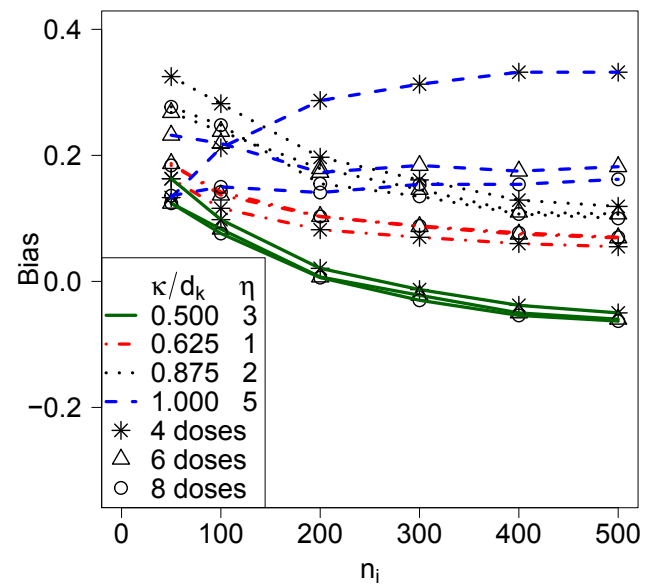

(a) Bias of SNCD0.67



(b) Bias of SNCD1.0

Figure 3.2: Variation of True Bias for SNCD0.67 and SNCD1.0 using Analytical Method

Figure 3.2 shows how the (true) bias of SNCD0.67 and SNCD1.0 change for these 72 scenarios. Overall pattern of these two plots looks similar. Therefore, it is clear that the relationship between the bias and these selected factors is the same for both SNCD0.67 and SNCD1.0. But the range of bias in panel (a) is $-0.1-0.4$ and that in panel (b) is $0.0-0.3$. Variability and size of bias is larger for SNCD0.67 than that for SNCD1.0. The reason could be that SNCD0.67 is estimated with 
smaller response than SNCD1.0. Consequently, validity of normality assumption is less satisfied, leading to a larger bias for the former.

Shapes of the Hill curves in the order of increasing bias are S-shaped with $\left(\kappa / d_{k}, \eta\right)=(0.5,3)$, convex with $\left(\kappa / d_{k}, \eta\right)=(0.625,1)$, concave with initial slope greater than zero with $\left(\kappa / d_{k}, \eta\right)=(0.875,2)$ and concave with initial slope zero with $\left(\kappa / d_{k}, \eta\right)=(1,5)$. In chapter 2 , we observe that the Hill model parameters are very well-estimated for S-shape curve; and only $\kappa$ is well-estimated for concave curve with initial slope zero specifically when four doses is used and significant improvement is observed when number of dose levels is increased. We see that this is reflected in the SNCD bias estimates for these curves.

The overall size of the bias decreases significantly with the increasing number of dose levels when this increase is from four to six rather than six to eight. This behavior is obvious for all except $\left(\kappa / d_{k}, \eta\right)=(1,5)$ combination. In chapter 2 , we observe similar pattern when investigating Hill model parameter estimates (Figures 2.6 and 2.8). Therefore, we can safely say that the characteristics observed in the Hill model parameter estimates are reflected here.

The size of the bias decreases with the increasing number of trials as well as increasing number of dose levels for all except for the concave curve with zero slope Hill curve. For this curve, bias increases with the increasing number of trials when the number of dose level is four; and stays almost the same with increasing the number of trials when the number of dose level is six or eight.

So, we can safely state that bias decreases more with increasing number of trials than increasing dose levels for S-shaped, convex and concave with initial slope greater than zero curves. As for the concave with initial slope zero curve, bias decreases significantly with increasing number of dose levels as opposed to increasing number of trials.

Figure 3.3 presents how the uncorrected and bias-corrected coverage probabili- 




(a) Uncorrected for $\mathrm{p}=0.67$

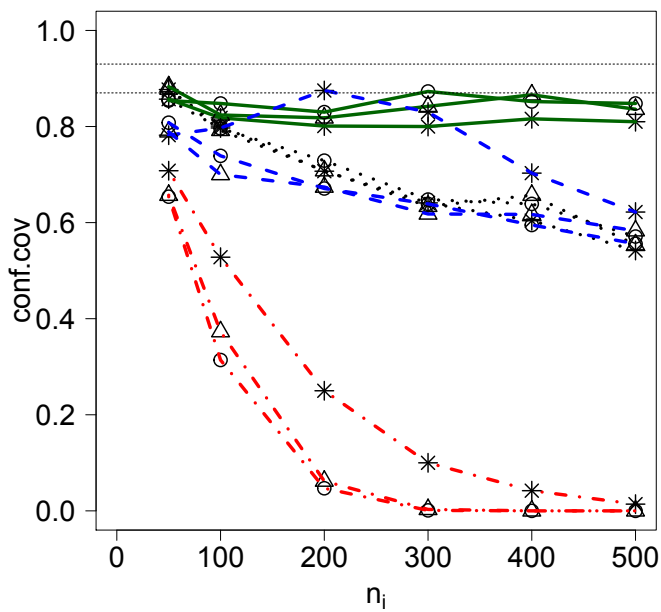

(c) Uncorrected for $\mathrm{p}=1.0$

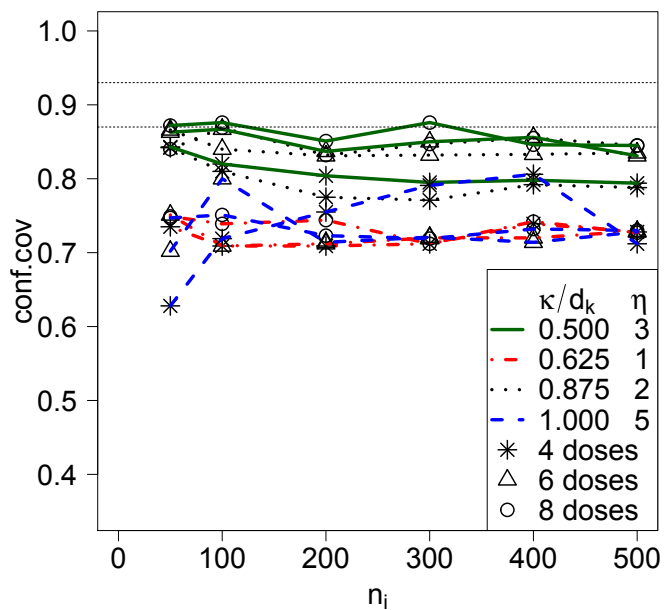

(b) Bias-corrected for $\mathrm{p}=0.67$



(d) Bias-corrected for $\mathrm{p}=1.0$

Figure 3.3: Variation of Coverage Probability based on (SNCDL, SNCDU) for SNCD0.67 and SNCD1.0 using Analytical Method

ties based on $(S N C D L, S N C D U)$ change with the shape of the Hill curves, number of dose levels and number of trials per dose. Pattern of the corresponding plots between SNCD0.67 and SNCD1.0 is similar but with coverage probability slightly more away from the nominal coverage band (given by the horizontal lines at 0.870 and 0.930 ) for SNCD0.67 than SNCD1.0 as expected. Overall, bias-corrected coverage probabilities 
(from panel (a) to (b) for SNCD0.67 and (c) to (d) for SNCD1.0) are significantly closer to the nominal coverage than the uncorrected coverage probabilities regardless of the shape of Hill curves, number of dose levels and number of trials. However, we observe that there may be a relationship between the shape of the Hill curves and the nearness of these probabilities to the nominal coverage.

Shapes of the Hill curves in order, where the coverage probabilities are close to the nominal, are S-shaped, concave with initial slope greater than zero, concave with initial slope zero and convex curve. We observe this same order for both the uncorrected and bias corrected probabilities but with significant improvement when bias-corrected. We recognize this same order of shapes when we investigate the relationship between the shape of the Hill curves and the parameter estimates in chapter 2. These results clearly state that accuracy and precision of the Hill model parameter estimates are reflected in the SNCD estimates.

For the S-shaped curve and concave with initial slope greater than zero, coverage probability is slightly more close to nominal when the number of dose levels is increased. But for other two shapes, opposite is true when number of dose level is increased from four to six; and no change from six to eight. These results seem to be counter-intuitive. However, the reason could be that when the number of dose levels is increased, width of the confidence interval (SNCDL, SNCDU) becomes narrower as expected, but size of the bias stays the same. As a result, proportion of (SNCDL, SNCDU) containing the true SNCD decreases with increasing the number of dose levels for these curves.

We observe that the changes in coverage probability for the specific shape and specific number of dose levels remains insignificant with the increasing number of trials per dose when bias-corrected. On the other hand, uncorrected coverage moves away from the nominal for all but S-shaped curve and more significantly for convex curve when number of trials per dose is increased. One possible explanation could 


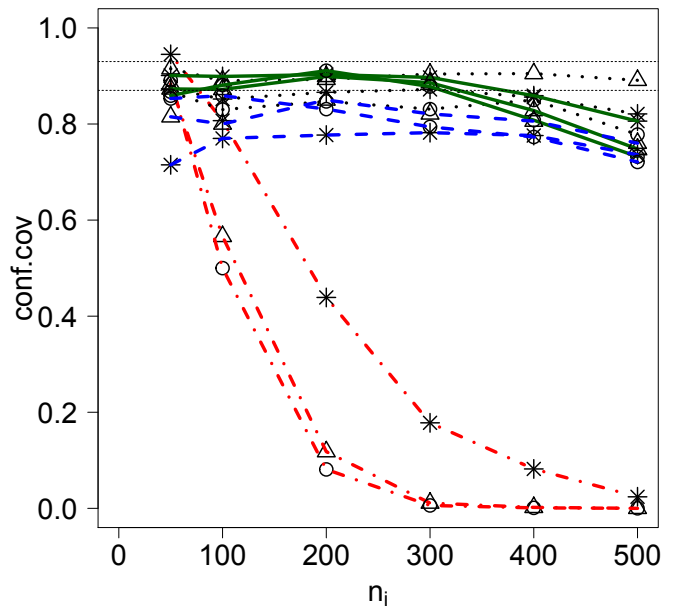

(a) Uncorrected for $\mathrm{p}=0.67$



(c) Uncorrected for $\mathrm{p}=1.0$

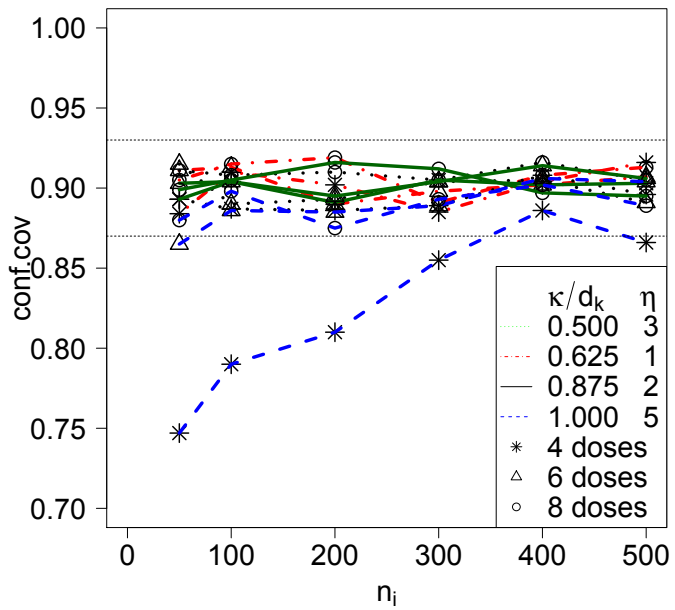

(b) Bias-corrected for $\mathrm{p}=0.67$

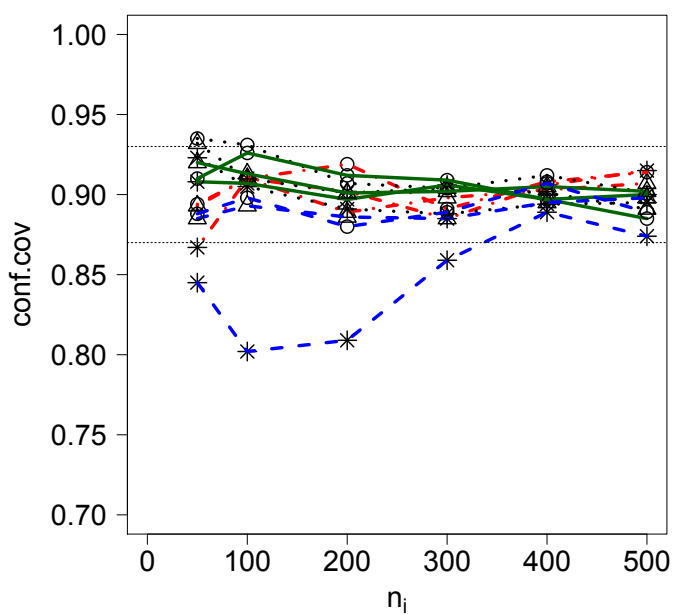

(d) Bias-corrected for $\mathrm{p}=1.0$

Figure 3.4: Variation of Coverage Probability based on $\left(S N C D_{05}, S N C D_{95}\right)$ for SNCD0.67 and SNCD1.0 using Analytical Method

be that width of the confidence interval decreases faster than the bias observed with increasing number of trials per dose.

Based on these results, we conclude that having number of dose levels of six as opposed to four improves the coverage probability. But, increasing the number of trials per dose is unnecessary when bias-corrected. Just as we observe in the results 
presented in chapter 2, we observe the best results for S-shaped curve, the second best for concave with initial slope greater than zero. Further, we observe that in chapter 2, the location parameter is poorly estimated for convex curve and the shape parameter for concave curve with initial slope zero. Here, we see that the coverage probability is far away from the nominal for the convex than to the concave with initial slope zero. One possible reason could be that the impact on the estimate of SNCD is significant from the estimate of $\kappa$ than that from $\eta$. Although, bias-correction significantly improves the coverage probability for all scenarios, none of the coverage probability falls within the nominal coverage of $(0.870,0.930)$. We report the results obtained by using the alternate confidence interval, $\left(S N C D_{05}, S N C D_{95}\right)$ (chapter 3.3.1 of page 48-49).

Figure 3.4 presents the uncorrected and bias corrected coverage probabilities based on $\left(S N C D_{05}, S N C D_{95}\right)$ for 72 scenarios considered here. Here, we observe a similar pattern to what we observe for coverage probabilities based on (SNCDL, SNCDU), but with probabilities much closer to nominal coverage in the case of uncorrected coverage and within the nominal for bias corrected coverage. Further, the pattern of changes in the coverage probabilities based on $\left(S N C D_{05}, S N C D_{95}\right)$ is same as that based on $(S N C D L, S N C D U)$ with the shape of the Hill curves, the number of dose levels and the number of trials per dose. Although the bias-corrected coverage probability for concave curve with initial slope zero with four dose levels falls outside the nominal coverage, we observe significant improvement as opposed to that based on $(S N C D L, S N C D U)$.

It is clear that the bias-corrected coverage based on $\left(S N C D_{05}, S N C D_{95}\right)$ falls within our nominal coverage regardless of the shape of the Hill curves, number of dose levels and number of trials per dose. These results confirm the importance of bias correction when estimates of SNCD are obtained by applying the analytical approach. Next, we investigate the similar results obtained by applying parametric 
and non-parametric bootstrap approach.

\subsubsection{Parametric Bootstrap Method}

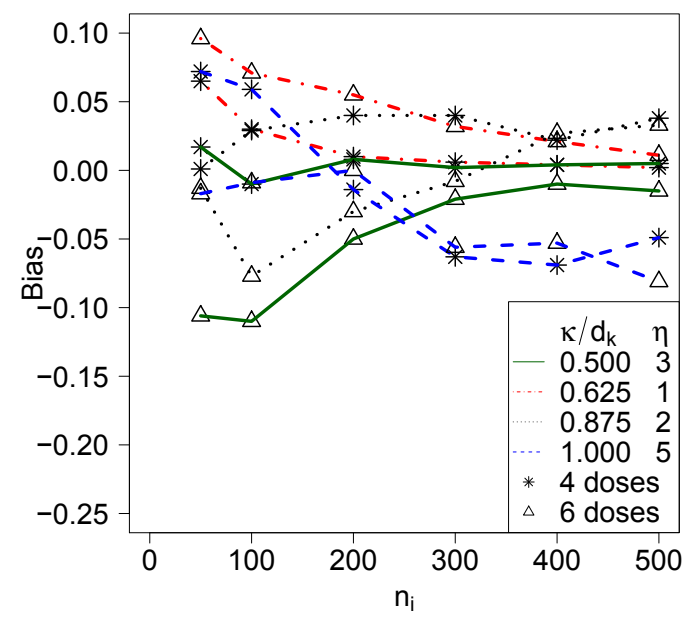

(a) Bias of SNCD0.67

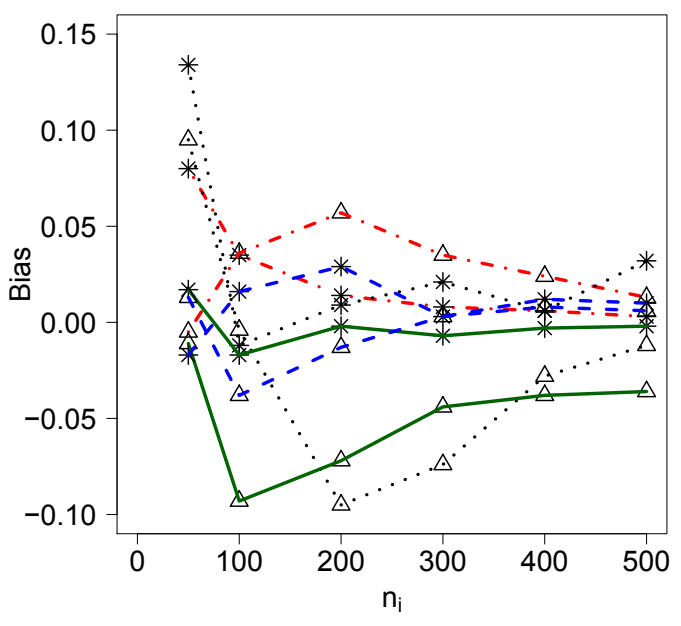

(b) Bias of SNCD1.0

Figure 3.5: Variation of True Bias for SNCD0.67 and SNCD1.0 using Parametric Bootstrap Method

We present the results obtained for bias, uncorrected and bias-corrected coverage probabilities for 48 different scenarios obtained from all possible combinations of four Hill model shapes, two different numbers of dose levels and six different numbers of trials per dose by applying the parametric bootstrap approach. As differences in the bias is insignificant when number of dose level is increased from six to eight, results are presented for only four and six doses. Range of bias is smaller than what we observe when using analytical approach. We present the bias observed when estimating SNCD of $\mathrm{p}=2 / 3$ and 1 .

Figure 3.5 presents the true bias of SNCD obtained by applying parametric bootstrap approach for 48 different scenarios. Overall pattern and range of the bias is almost same for SNCD0.67 and SNCD1.0. Pattern of the plots for biases obtained for S-shaped and concave with initial slope greater than zero overlaps more often 
than others; and largest bias is observed for convex curve and the second largest for concave with initial slope zero specially when number of trials per dose is the smallest. Here, we see similar pattern with less pronounced differences by the shape of the Hill model curves than that is observed when using analytical approach.

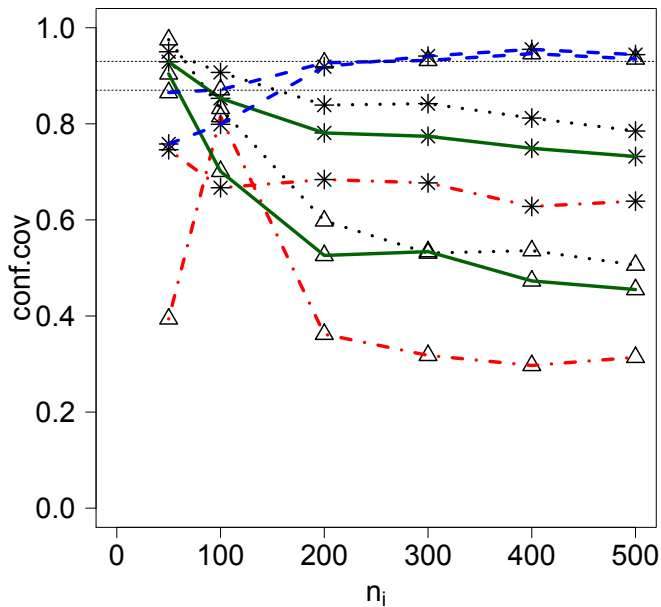

(a) Uncorrected for $\mathrm{p}=0.67$

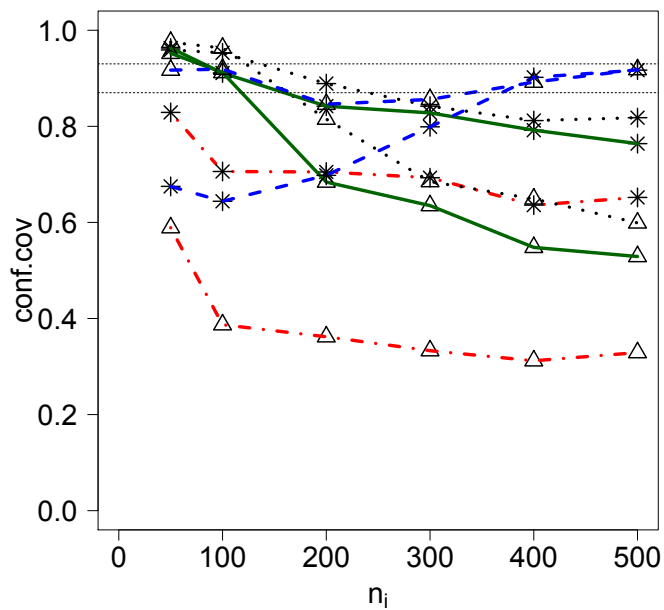

(c) Uncorrected for $\mathrm{p}=1.0$

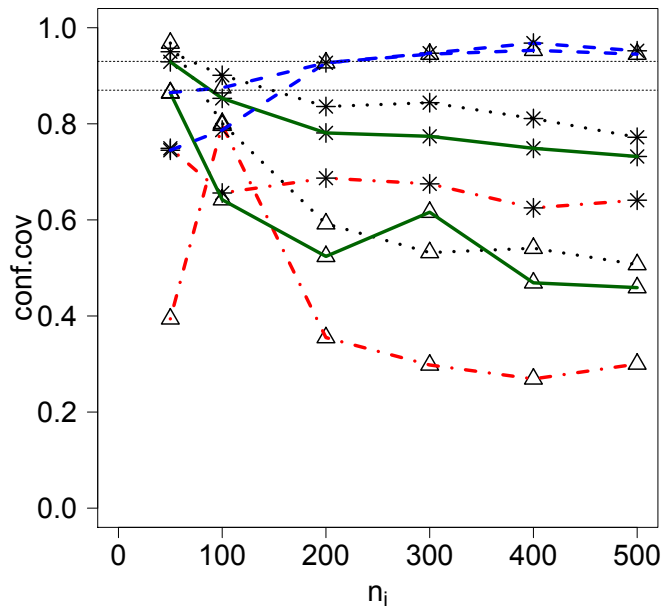

(b) Bias-corrected for $\mathrm{p}=0.67$

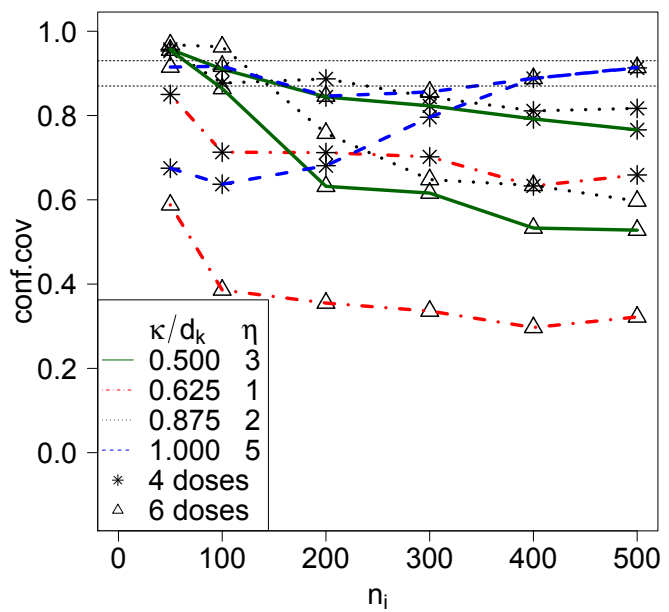

(d) Bias-corrected for $\mathrm{p}=1.0$

Figure 3.6: Variation of Coverage Probability based on (SNCDL, SNCDU) for SNCD0.67 and SNCD1.0 using Parametric Bootstrap Method

We see that increasing the number of dose levels decreases the bias and goes in 
the direction of negative bias for all except convex curve. In the case of convex curve, bias is larger when the number of dose level is six. We observe no obvious pattern between the bias and the number of dose levels.

Overall the range of bias decreases and hovers around more close to zero when number of trials per dose increases. We observe a significant decrease in the bias when number of trials per dose is 500 regardless of the shape of the Hill curves and number of dose levels. However, there is no noticeable relationship between bias and the three factors considered - shape of the Hill curves, number of dose levels and number of trials per dose.

Figure 3.6 presents the uncorrected and bias-corrected coverage probability for all 48 different scenarios. Pattern of uncorrected and bias-corrected coverage probability based on (SNCDL, SNCDU) is similar for SNCD0.67 and SNCD1.0 (panel (a) versus panel (c) and panel (b) versus panel (d)). Bias correction does not improve the coverage probability for either SNCD0.67 or SNCD1.0 (panel (a) versus panel (d) for SNCD0.67 and panel (c) versus panel (d) for SNCD1.0).

Variability of uncorrected coverage probability in the 48 different scenarios is smaller than that obtained using analytical approach. This uncorrected coverage probability is far from nominal compared to that obtained from analytical approach for S-shaped and concave with initial slope greater than zero. Coverage is very close to nominal for concave with initial slope zero. There is no improvement in the coverage probability for any when bias correction is done.

Increasing the number of dose levels decreases the coverage for corresponding shape of the Hill curves and number of trials per dose except for concave with initial slope zero for both uncorrected and bias-corrected coverage.

We observe insignificant change in the coverage probability as the number of trials per dose increases for corresponding number of dose levels and for all shape of the Hill curves except concave with initial slope zero. In this case, specially for 
SNCD1.0, coverage improves with increasing number of trials per dose when number of dose level is four.

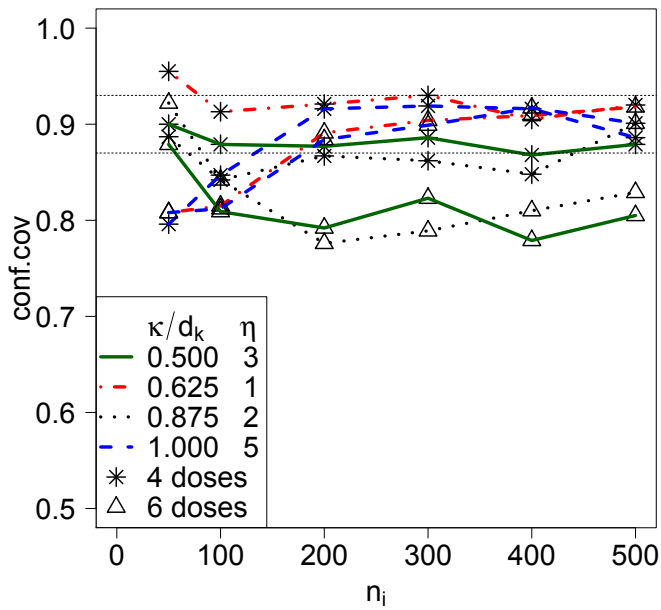

(a) Uncorrected for $\mathrm{p}=0.67$

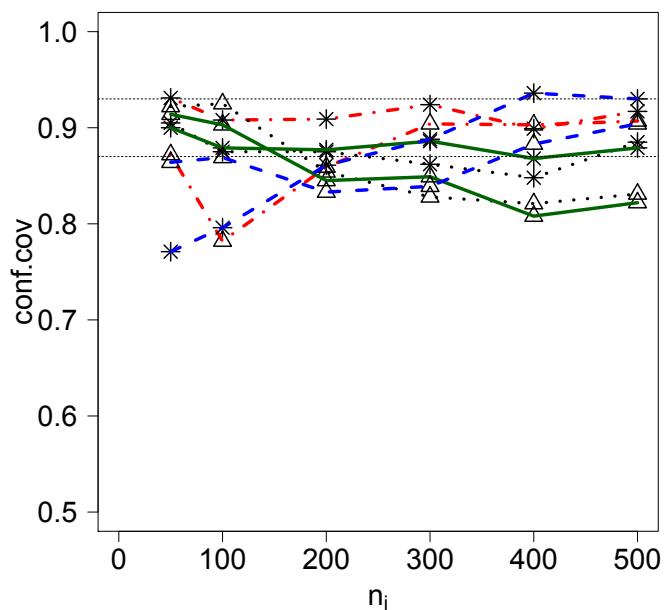

(c) Uncorrected for $\mathrm{p}=1.0$

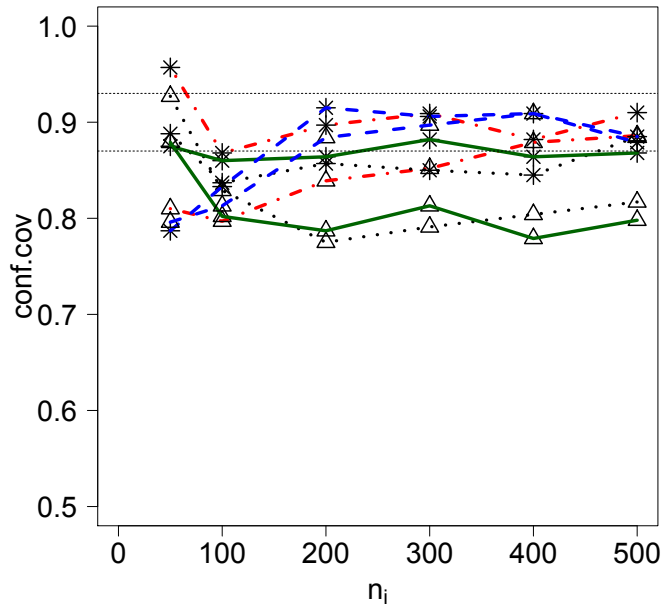

(b) Bias-corrected for $\mathrm{p}=0.67$

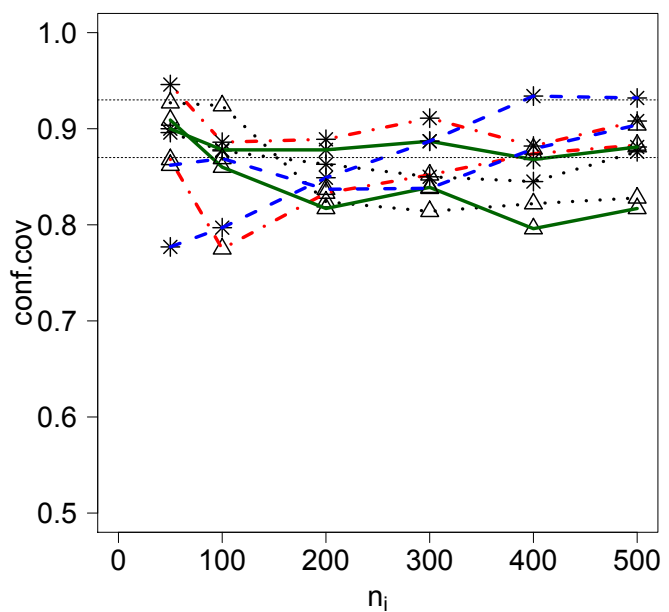

(d) Bias-corrected for $\mathrm{p}=1.0$

Figure 3.7: Variation of Coverage Probability based on $\left(S N C D_{05}, S N C D_{95}\right)$ for SNCD0.67 and SNCD1.0 using Parametric Bootstrap Method

Based on these observations, we can conclude that neither bias-correction, increasing the number of dose levels, changing the shape of the Hill model curves nor increasing the number of trials per dose have an impact on the coverage probabilities 
on all 48 different scenarios. Overall performance is better when bias-corrected analytical approach is used as opposed to either uncorrected or bias-corrected parametric bootstrap approach based on (SNCDL, SNCDU) is used. Next we discuss the similar results for coverage probabilities obtained for the alternative confidence interval, $\left(S N C D_{05}, S N C D_{95}\right)$.

Figure 3.7 presents the uncorrected and bias corrected probabilities based on $\left(S N C D_{05}, S N C D_{95}\right)$ for 48 different scenarios discussed above. There are no significant differences in the range or pattern of uncorrected and bias-corrected coverage probabilities regardless of whether it is for SNCD0.67 of SNCD1.0. Uncorrected or bias-corrected coverage probability for all the scenarios are above 0.75 which is significantly higher than what we observe for coverage probability based on (SNCDL, SNCDU).

We observe that overall coverage probability falls within the nominal range for convex and concave with initial slope zero curve regardless of whether it is uncorrected or bias-corrected; or for SNCD0.67 or SNCD1.0. We see that increasing the number of dose level decreases the coverage probability in all four panels. This effect is worse for S-shaped and concave with initial slope greater than zero. However, there is no change in the coverage probability with increasing number of trials per dose for corresponding number of dose levels and the shape of the Hill curves.

Hence, we can conclude that coverage based on $\left(S N C D_{05}, S N C D_{95}\right)$ is better than that based on (SNCDL, SNCDU) when parametric bootstrap approach is used. Uncorrected coverage probability obtained by applying parametric bootstrap approach is closer to nominal than that obtained by applying analytical approach. But, when bias-corrected, coverage probabilities obtained by using analytical approach for both types of confidence interval are better than that obtained by using parametric approach. 


\subsubsection{Non-parametric Bootstrap Method}

We first present the bias observed in SNCD0.67 and SNCD1.0 when nonparametric bootstrap approach is applied for all 48 different scenarios mentioned above (Simulation results are obtained for 72 different scenarios mentioned in the analytical approach section. But, we present the results for 48 scenarios as we observe no improvement in the results while dose levels is increased from six to eight). In the case of bias, we observe pattern similar to when using parametric bootstrap approach. There is no obvious relationship between the bias and the shape of Hill curves, number of dose levels and number of trials per dose.

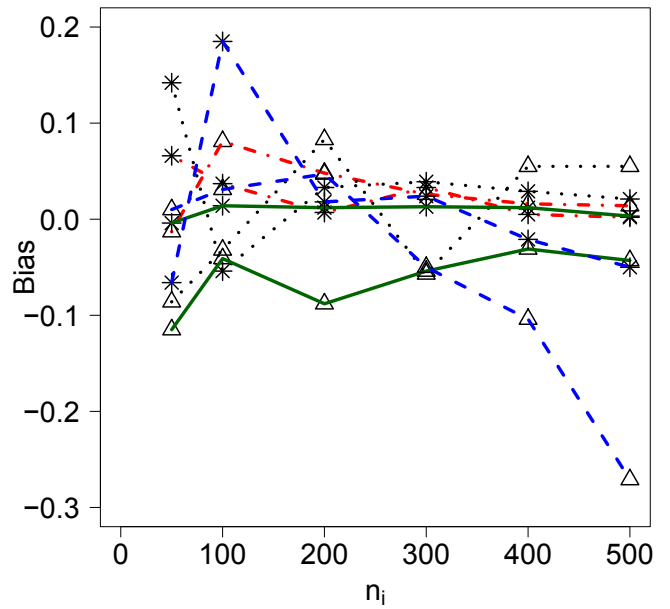

(a) Bias of SNCD0.67

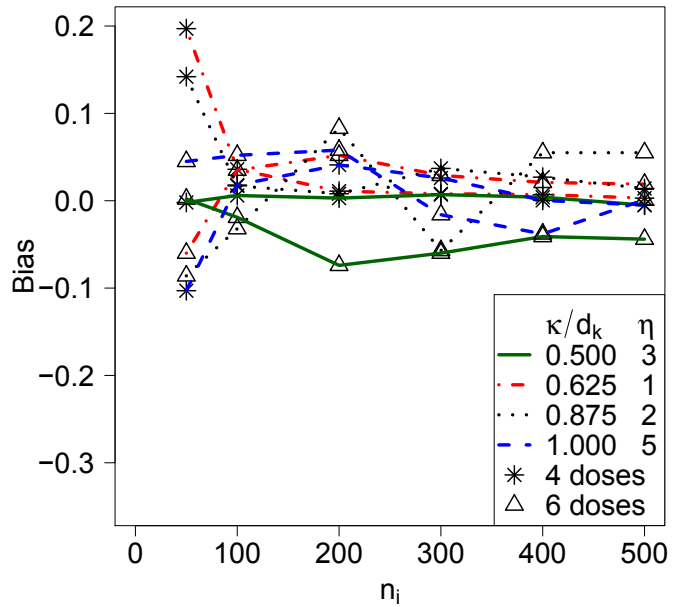

(b) Bias of SNCD1.0

Figure 3.8: Variation of True Bias for SNCD0.67 and SNCD1.0 using Non-parametric Bootstrap Method

Figure 3.9 includes the uncorrected and bias-corrected coverage probability based on (SNCDL, SNCDU) for SNCD0.67 and SNCD1.0 for all 48 different scenarios considered here. We see that bias correction has no impact on this coverage probability either for SNCD0.67 or SNCD1.0. The trend that we point out for parametric bootstrap approach uncorrected or bias-corrected coverage probability, is observed here for corresponding shape of the Hill curve, number of dose levels and number of 
trials per dose.

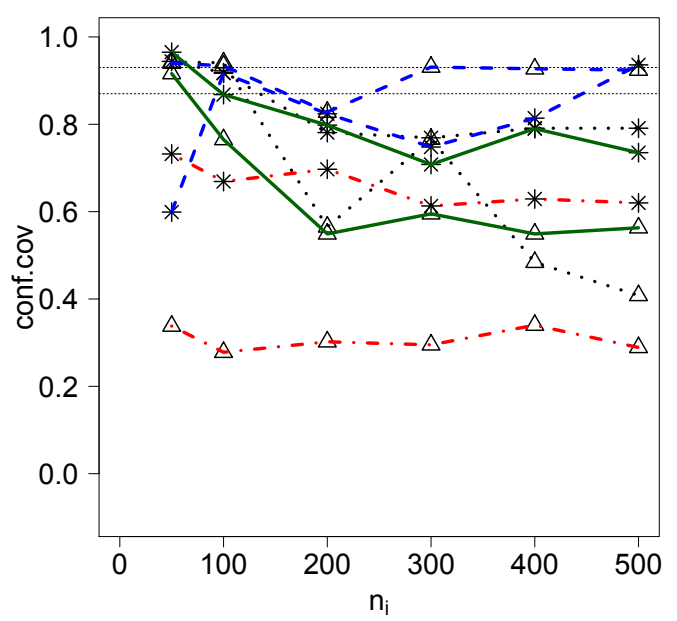

(a) Uncorrected for $\mathrm{p}=0.67$

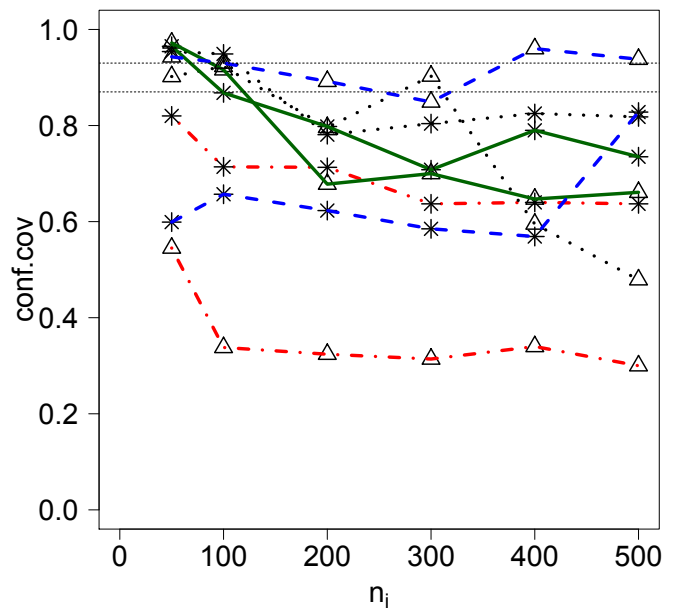

(c) Uncorrected for $\mathrm{p}=1.0$

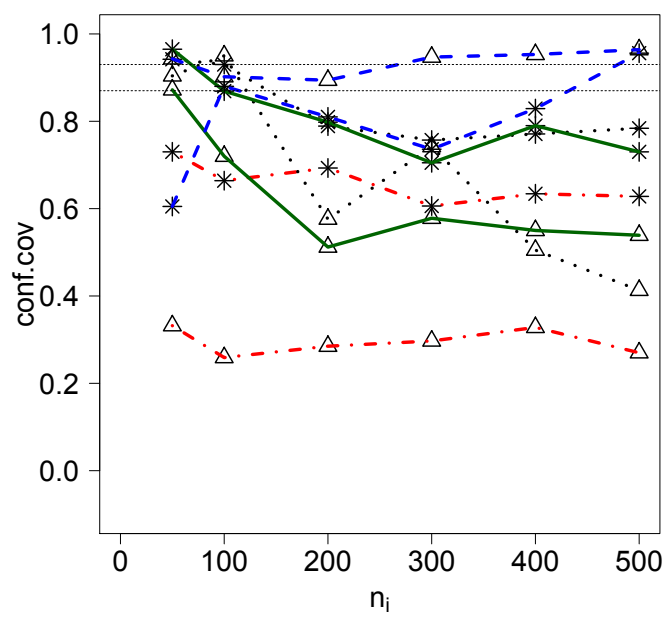

(b) Bias-corrected for $\mathrm{p}=0.67$



(d) Bias-corrected for $\mathrm{p}=1.0$

Figure 3.9: Variation of Coverage Probability based on (SNCDL, SNCDU) for SNCD0.67 and SNCD1.0 using Non-parametric Bootstrap Method

Figure 3.10 presents uncorrected and bias-corrected coverage probability based on $\left(S N C D_{05}, S N C D_{95}\right)$ confidence interval. Just as we observe in the case of parametric bootstrap approach, we see that coverage probability is significantly close to the nominal range when this confidence interval is used as opposed to (SNCDL, 
SNCDU) confidence interval. Variability in the curve is smaller for corresponding panels when coverage probability is investigated for SNCD1.0 here. Further, there is no improvement in the coverage when the bias correction is applied.

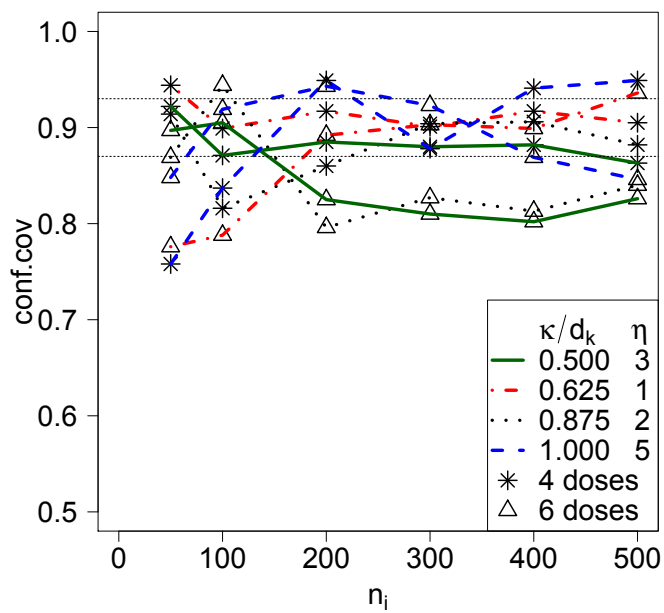

(a) Uncorrected for $\mathrm{p}=0.67$

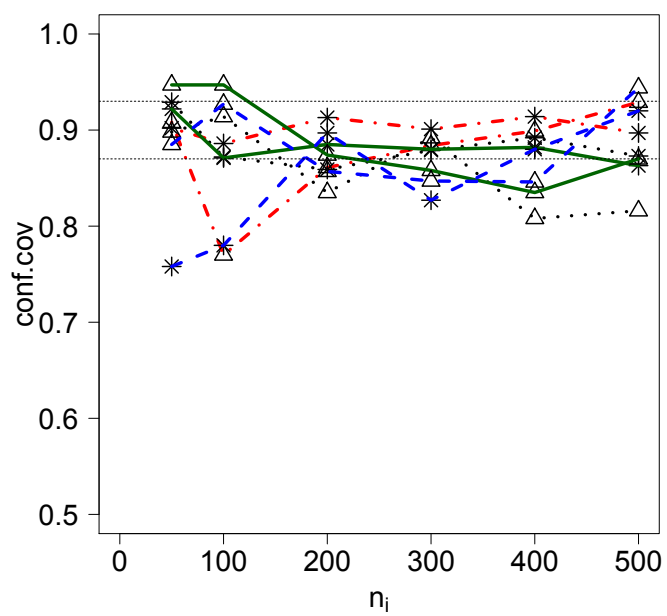

(c) Uncorrected for $\mathrm{p}=1.0$

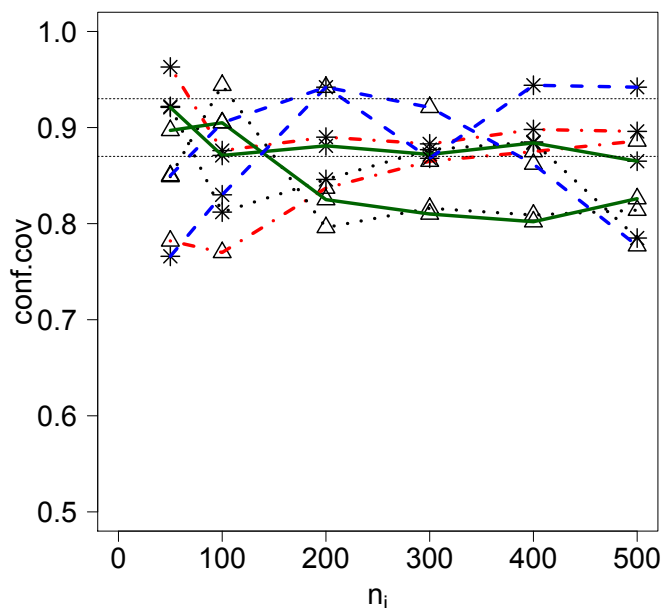

(b) Bias-corrected for $\mathrm{p}=0.67$

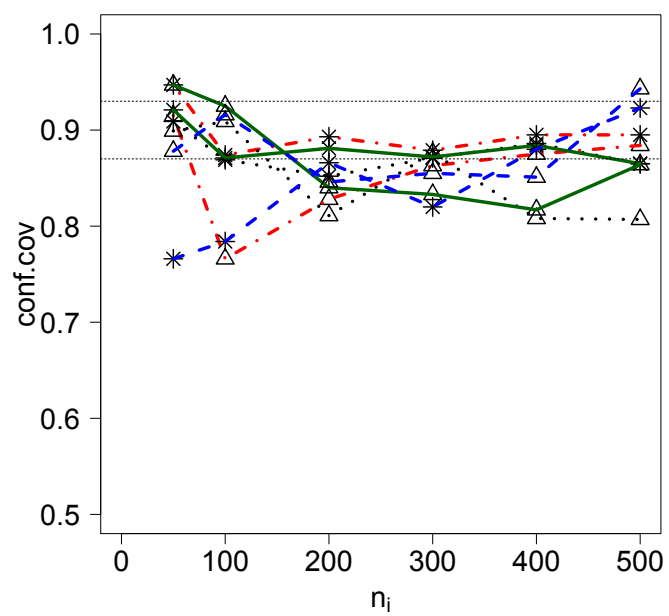

(d) Bias-corrected for $\mathrm{p}=1.0$

Figure 3.10: Variation of Coverage Probability based on $\left(S N C D_{05}, S N C D_{95}\right)$ for SNCD0.67 and SNCD1.0 using Non-parametric Bootstrap Method

Overall we can safely state that uncorrected coverage probability is close to nominal range when parametric or non-parametric approach is used over analytical 
approach for both (SNCDL, SNCDU) and $\left(S N C D_{05}, S N C D_{95}\right)$. On the other hand, analytical approach perform the best when bias-correction is applied. These coverage probabilities are closer to nominal for both SNCD1.0 and SNCD0.67. However, bias observed in the analytical approach is larger than that observed in either parametric or non-parametric approach. We investigate the relationship between these measures; bias, uncorrected and bias-corrected coverage probability based on (SNCDL, SNCDU) and $\left(S N C D_{05}, S N C D_{95}\right)$; and the values of Hill model parameters, number of dose levels and number of trials per dose when applying analytical approach in the context of multiple regression.

\subsubsection{Multiple Regression}

We investigate how bias, uncorrected and bias-corrected coverage probabilities based on $(S N C D L, S N C D U)$ and $\left(S N C D_{05}, S N C D_{95}\right)$ change depending on the the shape of the Hill model curves, number of dose levels and number of trials. To confirm this relationship using multiple regression analysis, we further estimate bias, uncorrected and bias corrected coverage probabilities based on $(S N C D L, S N C D U)$ and $\left(S N C D_{05}, S N C D_{95}\right)$ for $\left(\kappa / d_{k}, \eta\right)=((0.50,1),(0.50,2),(0.875,1),(0.875,3)$, $(0.875,4),(1.00,2),(1.125,3),(1.125,4),(1.250,1),(1.250,2),(1.250,4),(1.250$, 7)) with background incident fixed at $\alpha=0.01$, with the three different dose levels, six different number of trials per dose.

\subsubsection{Qualitative Explanatory Variables}

Here, we identify the relationship between the bias, uncorrected and biascorrected coverage probabilities based on $(S N C D L, S N C D U)$ or $\left(S N C D_{05}, S N C D_{95}\right)$ as response variables with the selected Hill model parameter values $-\kappa$ and $\eta$ with fixed $\alpha$ at 0.01 , number of dose levels, number of trials per dose as qualitative explanatory variables. Selected $\kappa$ values as indicator variables are 4, 5, 7, 8, 9 and 10; 
the corresponding $\eta$ values as indicator variables $1,2,3,4,5$ and 6 ; number of dose levels as indicator variables 4, 6 and 8; and number of trials per dose as indicator variables 50,100, 200, 300, 400 and 500. Multiple regression equation used to identify this relationship is given by,

$$
\begin{aligned}
y= & \beta_{0}+\beta_{1} \mathbb{1}_{k a 1}+\beta_{2} \mathbb{1}_{k a 2}+\beta_{3} \mathbb{1}_{k a 3}+\beta_{4} \mathbb{1}_{k a 4}+\beta_{5} \mathbb{1}_{k a 5}+\alpha_{1} \mathbb{1}_{e t 1}+\alpha_{2} \mathbb{1}_{e t 2}+\alpha_{3} \mathbb{1}_{e t 3}+\alpha_{4} \mathbb{1}_{e t 4} \\
& +\alpha_{5} \mathbb{1}_{e t 5}+\theta_{1} \mathbb{1}_{d 1}+\theta_{2} \mathbb{1}_{d 2}+\gamma_{1} \mathbb{1}_{n 1}+\gamma_{2} \mathbb{1}_{n 2}+\gamma_{3} \mathbb{1}_{n 3}+\gamma_{4} \mathbb{1}_{n 4}+\gamma_{5} \mathbb{1}_{n 5}+\epsilon_{2}
\end{aligned}
$$

where

$$
\begin{aligned}
& \mathbb{1}_{k a 1}= \begin{cases}1 & \text { if } \kappa=4 \\
0 & \text { if } \mathrm{o} / \mathrm{w}\end{cases} \\
& \mathbb{1}_{k a 2}= \begin{cases}1 & \text { if } \kappa=5 ; \\
0 & \mathrm{o} / \mathrm{w}\end{cases} \\
& \mathbb{1}_{k a 3}= \begin{cases}1 & \text { if } \kappa=7 \\
0 & \mathrm{o} / \mathrm{w}\end{cases} \\
& \mathbb{1}_{k a 4}= \begin{cases}1 & \text { if } \kappa=8 \\
0 & \text { if o/w. }\end{cases} \\
& \mathbb{1}_{\text {ka5 }}= \begin{cases}1 & \text { if } \kappa=9 ; \\
0 & \text { if } \mathrm{o} / \mathrm{w} .\end{cases} \\
& \mathbb{1}_{\text {et } 1}= \begin{cases}1 & \text { if } \eta=1 \\
0 & \mathrm{o} / \mathrm{w}\end{cases} \\
& \mathbb{1}_{\text {et } 2}= \begin{cases}1 & \text { if } \eta=2 \\
0 & \mathrm{o} / \mathrm{w}\end{cases} \\
& \mathbb{1}_{e t 3}= \begin{cases}1 & \text { if } \eta=3 \\
0 & \text { if } \mathrm{o} / \mathrm{w} .\end{cases} \\
& \mathbb{1}_{\text {et4 }}= \begin{cases}1 & \text { if } \eta=4 \\
0 & \mathrm{o} / \mathrm{w} .\end{cases} \\
& \mathbb{1}_{\text {et } 5}= \begin{cases}1 & \text { if } \eta=5 \\
0 & \mathrm{o} / \mathrm{w}\end{cases} \\
& \mathbb{1}_{d 1}= \begin{cases}1 & \text { if } \# \text { doses }=4 \\
0 & \text { if } \mathrm{o} / \mathrm{w}\end{cases} \\
& \mathbb{1}_{d 2}= \begin{cases}1 & \text { if } \# \text { doses }=6 ; \\
0 & \text { o } / \mathrm{w} .\end{cases} \\
& \mathbb{1}_{n 1}= \begin{cases}1 & \text { if } n=50 \\
0 & \mathrm{o} / \mathrm{w}\end{cases} \\
& \mathbb{1}_{n 2}= \begin{cases}1 & \text { if } n=100 \\
0 & \mathrm{o} / \mathrm{w}\end{cases} \\
& \mathbb{1}_{n 3}= \begin{cases}1 & \text { if } n=200 \\
0 & \mathrm{o} / \mathrm{w}\end{cases} \\
& \mathbb{1}_{n 4}= \begin{cases}1 & \text { if } n=300 \\
0 & \mathrm{o} / \mathrm{w}\end{cases}
\end{aligned}
$$




$$
\mathbb{1}_{n 5}= \begin{cases}1 & \text { if } n=400 \\ 0 & \mathrm{o} / \mathrm{w}\end{cases}
$$

Appropriate reduced multiple regression equation, Model I, is derived using partial F test on location parameters, shape parameters, numbers of dose levels and numbers of trials per dose as groups; and Model II, is derived using partial F test for individual values of location and shape parameters, numbers of dose levels and numbers of trials per dose. We perform an appropriated tests of hypothesis for intercept of these multiple regression equations. The intercepts of the reduced models for these four different coverage probabilities as response variables are tested for 0.9 .

$$
\begin{aligned}
& H_{0}: \beta_{0}=0.9 \\
& H_{1}: \beta_{0} \neq 0.9
\end{aligned}
$$

The intercept of the reduced models for bias as response variables is tested for zero.

$$
\begin{aligned}
& H_{0}: \beta_{0}=0 \\
& H_{1}: \beta_{0} \neq 0
\end{aligned}
$$

\section{Analytical Approach Results}

Reduced multiple regression equations for bias, uncorrected and bias-corrected coverage probabilities based on (SNCDL, SNCDU) and $\left(S N C D_{05}, S N C D_{95}\right)$ as response variables are derived for SNCD0.67 and SNCD1.0 as explained in section 3.5.2.1.

We carry out the hypothesis test on the intercept of multiple regression equations for bias, uncorrected and bias-corrected coverage probability based on (SNCDL, SNCDU) and $\left(S N C D_{05}, S N C D_{95}\right)$. Intercept for bias is tested using 3.45) and that for coverage using 3.44. First, we discuss these multiple regression equations obtained for SNCD0.67 and SNCD1.0. 


\begin{tabular}{|c|c|c|c|c|c|}
\hline & \multicolumn{5}{|c|}{ Model I } \\
\hline & $C c P b_{0595}$ & $c P b_{0595}$ & $C c P b_{L U}$ & $c P b_{L U}$ & Bias \\
\hline$\beta_{0}$ & 0.898 & 0.660 & 0.676 & 0.414 & -0.01 \\
\hline$\beta_{1}$ & - & -0.1 & - & -0.1 & -0.3 \\
\hline$\beta_{2}$ & - & -0.2 & - & -0.2 & -0.2 \\
\hline$\beta_{3}$ & - & -0.1 & - & -0.2 & -0.1 \\
\hline$\beta_{4}$ & - & -0.1 & - & -0.1 & -0.1 \\
\hline$\beta_{5}$ & - & -0.2 & - & -0.2 & - \\
\hline$\alpha_{1}$ & - & -0.3 & 0.1 & -0.2 & 0.3 \\
\hline$\alpha_{2}$ & - & 0.1 & 0.2 & 0.2 & 0.3 \\
\hline$\alpha_{3}$ & - & 0.1 & 0.2 & 0.3 & 0.3 \\
\hline$\alpha_{4}$ & - & 0.1 & 0.1 & 0.3 & 0.3 \\
\hline$\alpha_{5}$ & - & 0.1 & - & 0.2 & 0.2 \\
\hline$\theta_{1}$ & - & - & - & - & - \\
\hline$\theta_{2}$ & - & - & - & - & - \\
\hline$\gamma_{1}$ & - & 0.1 & - & 0.3 & - \\
\hline$\gamma_{2}$ & - & 0.3 & - & 0.3 & - \\
\hline$\gamma_{3}$ & - & 0.2 & - & 0.2 & - \\
\hline$\gamma_{4}$ & - & 0.1 & - & 01 & - \\
\hline \multirow[t]{2}{*}{$\gamma_{5}$} & - & - & - & 0.1 & - \\
\hline & \multicolumn{5}{|c|}{ Model II } \\
\hline$\overline{\beta_{0}}$ & 0.895 & 0.690 & 0.674 & 0.437 & 0.183 \\
\hline$\beta_{1}$ & - & - & - & - & -0.2 \\
\hline$\beta_{2}$ & - & -0.2 & - & -0.1 & -0.1 \\
\hline$\beta_{3}$ & - & - & -0.1 & - & -0.1 \\
\hline$\beta_{4}$ & - & - & - & - & - \\
\hline$\beta_{5}$ & - & -0.1 & - & -0.1 & 0.1 \\
\hline$\alpha_{1}$ & - & -0.4 & 0.1 & -0.3 & - \\
\hline$\alpha_{2}$ & - & - & 0.2 & 0.2 & 0.1 \\
\hline$\alpha_{3}$ & - & - & 0.2 & 0.1 & 0.1 \\
\hline$\alpha_{4}$ & - & - & 0.1 & 0.2 & - \\
\hline$\alpha_{5}$ & - & - & - & 0.1 & - \\
\hline$\theta_{1}$ & - & - & - & - & - \\
\hline$\theta_{2}$ & - & - & - & - & - \\
\hline$\gamma_{1}$ & - & 0.3 & - & 0.3 & 0.1 \\
\hline$\gamma_{2}$ & - & 0.2 & - & 0.2 & - \\
\hline$\gamma_{3}$ & - & 0.1 & - & 0.1 & - \\
\hline$\gamma_{4}$ & - & - & - & 0.1 & - \\
\hline$\gamma_{5}$ & - & - & - & - & - \\
\hline
\end{tabular}

Table 3.1: Multiple Regression Parameter Estimates (Analytical) when $p=0.67$ 


\begin{tabular}{|c|c|c|c|c|c|}
\hline & \multicolumn{5}{|c|}{ Model I } \\
\hline & $C c P b_{0595}$ & $c P b_{0595}$ & $C c P b_{L U}$ & $c P b_{L U}$ & Bias \\
\hline$\beta_{0}$ & 0.895 & 0.622 & 0.783 & 506 & -0.025 \\
\hline$\beta_{1}$ & - & - & - & 0.1 & -0.2 \\
\hline$\beta_{2}$ & - & - & - & - & -0.1 \\
\hline$\beta_{3}$ & - & - & - & - & -0.1 \\
\hline$\beta_{4}$ & - & - & - & - & -0.1 \\
\hline$\beta_{5}$ & - & - & - & -0.1 & - \\
\hline$\alpha_{1}$ & - & -0.4 & -0.1 & -0.5 & 0.3 \\
\hline$\alpha_{2}$ & - & - & - & - & 0.3 \\
\hline$\alpha_{3}$ & - & - & - & - & 0.3 \\
\hline$\alpha_{4}$ & - & - & - & - & 0.3 \\
\hline$\alpha_{5}$ & - & - & -0.1 & - & 0.2 \\
\hline$\theta_{1}$ & - & - & - & - & - \\
\hline$\theta_{2}$ & - & - & - & - & - \\
\hline$\gamma_{1}$ & - & 0.4 & 0.1 & 0.4 & - \\
\hline$\gamma_{2}$ & - & 0.3 & - & 0.3 & - \\
\hline$\gamma_{3}$ & - & 0.2 & - & 0.2 & - \\
\hline$\gamma_{4}$ & - & 0.1 & - & 0.1 & - \\
\hline \multirow[t]{2}{*}{$\gamma_{5}$} & - & 0.1 & - & 0.1 & - \\
\hline & \multicolumn{5}{|c|}{ Model II } \\
\hline$\overline{\beta_{0}}$ & 0.900 & 0.640 & 0.759 & 0.531 & 0.155 \\
\hline$\beta_{1}$ & - & - & - & - & -0.1 \\
\hline$\beta_{2}$ & - & -0.1 & - & -0.1 & - \\
\hline$\beta_{3}$ & - & - & - & - & - \\
\hline$\beta_{4}$ & - & -12 & - & -1 & - \\
\hline$\beta_{5}$ & - & -0.1 & - & -0.1 & - \\
\hline$\alpha_{1}$ & - & -0.4 & - & -0.5 & - \\
\hline$\alpha_{2}$ & - & - & 0.1 & - & 0.1 \\
\hline$\alpha_{3}$ & - & - & 0.1 & - & 0.1 \\
\hline$\alpha_{4}$ & - & - & - & - & 0.1 \\
\hline$\alpha_{5}$ & - & - & - & - & - \\
\hline$\theta_{1}$ & - & 0.1 & - & - & - \\
\hline$\theta_{2}$ & - & - & - & - & - \\
\hline$\overline{\gamma_{1}}$ & - & $\bar{~} 0.3$ & $\bar{~} 0.1$ & $\overline{0.4}$ & - \\
\hline$\gamma_{2}$ & - & 0.3 & - & 0.3 & - \\
\hline$\gamma_{3}$ & - & 0.2 & - & 0.2 & - \\
\hline$\gamma_{4}$ & - & 0.1 & - & 0.1 & - \\
\hline$\gamma_{5}$ & - & - & - & - & - \\
\hline
\end{tabular}

Table 3.2: Multiple Regression Parameter Estimates (Analytical) when $p=1.0$ 
Tables 3.1 and 3.2 present estimates of the multiple regression parameters derived for bias, uncorrected and bias-corrected coverage probabilities for analytically estimated SNCD0.67 and SNCD1.0. These regression parameter estimates for bias of SNCD0.67 and SNCD1.0 confirm what we discuss in chapter 3.5.1.1 based on Figure 3.2. We observe a similarity in the regression parameter estimates for SNCD0.67 and SNCD1.0, but with a few more significant regression parameters for bias of SNCD0.67 as expected, as calculation of SNCD0.67 carried out with response closer to zero than that for SNCD1.0. These equations for biases are mostly governed by the Hill model's location and shape parameter than number of dose levels or number of trials per dose. This again confirms what we observe in Figure 3.2.

We investigate the regression equations obtained for uncorrected and biascorrected coverage probabilities based on (SNCDL, SNCDU). Results in Tables 3.1 and 3.2 confirm what we observe and discuss from Figure 3.6. Both of these coverage probabilities based on (SNCDL, SNCDU) for SNCD0.67 are more sensitive to the selected explanatory variables than that for SNCD1.0. Uncorrected coverage probability depends on the Hill model parameters and number of trials per dose than number of dose levels. However, when bias-correction is done, this coverage probability is no longer a function of number of trials per dose. We observe similar results in Figure 3.6.

Next, we discuss the regression equations obtained for uncorrected and biascorrected coverage probabilities based on $\left(S N C D_{05}, S N C D_{95}\right)$. We investigate the results presented in Tables 3.1 and 3.2 for estimates of regression parameters for uncorrected and bias-corrected coverage probabilities for both SNCD0.67 and SNCD1.0. First, we look at characteristics of regression equation for uncorrected coverage. We realize that it is a function of location and shape parameters of the Hill model and number of trials per dose for SNCD0.67, but less dependent on the Hill model parameters for coverage for SNCD1.0. We identify similar relationship in Figure 3.7 
of panel (a) and (c). Clearly, we observe that bias-corrected coverage probability is independent of any of the explanatory variables investigated as expected.

\begin{tabular}{|c||c|c|}
\hline P.value & Model1 & Model2 \\
\hline \hline$C c P b_{0595}$ & 0.736 & 0.08 \\
\hline$c P b_{0595}$ & 0.00 & 0.00 \\
\hline$C c P b_{L U}$ & 0.00 & 0.00 \\
\hline$c P b_{L U}$ & 0.00 & 0.00 \\
\hline Bias $_{S N C D}$ & 0.592 & 0.00 \\
\hline \hline
\end{tabular}

Table 3.3: Analytical Approach P-values for $p=0.67$

\begin{tabular}{|c||c|c|}
\hline P.value & Model1 & Model2 \\
\hline \hline$C c P b_{0595}$ & 0.374 & 0.453 \\
\hline$c P b_{0595}$ & $3.0 \mathrm{e}-9$ & 0.000 \\
\hline$C c P b_{L U}$ & 0.000 & 0.000 \\
\hline$c P b_{L U}$ & 0.000 & 0.000 \\
\hline Bias $_{S N C D}$ & 0.210 & 0.000 \\
\hline \hline
\end{tabular}

Table 3.4: Analytical Approach P-values for $p=1.0$

Tables 3.3 presents the p-values for the tests of hypothesis performed on the intercepts of multiple regression equations derived for bias, uncorrected and biascorrected coverage probabilities for SNCD0.67. Table 3.4 presents the same results for SNCD1.0. We observe that p-values of bias-corrected coverage probabilities based on $\left(S N C D_{05}, S N C D_{95}\right)$ for SNCD0.67 and SNCD1.0 are very high. By combining the corresponding estimates observed in Tables 3.1 and 3.2, we clearly see that this coverage probability is 0.9 and is independent of any of the explanatory variables considered. On the contrary, all the other three coverage probabilities estimates are function of some of the explanatory variables as shown in Tables 3.1 and 3.2. And we reject the hypothesis that intercept is 0.9 for all these cases. This again confirms that bias-corrected coverage probabilities based on (SNCDL, SNCDU) fails to fall within the nominal coverage. For model I, we accept the test of hypothesis for the intercept of regression equation for the bias, but regression equation depends on the 
explanatory variables considered. On the contrary for model II, we reject this same hypothesis, but the regression equation is a function of only few variables considered. This confirms that there is a bias in the analytical approach estimation. We derive similar multiple regression equations for these five response variables using bootstrap approach.

\section{Parametric Bootstrap Approach Results}

We present the parameter estimates for multiple regression equations derived for bias, uncorrected and bias-corrected probabilities based on (SNCDL, SNCDU) and $\left(S N C D_{05}, S N C D_{95}\right)$ in Tables 3.5 and 3.6; and p-values for the hypothesis test on the intercept of these multiple regression equation in Tables 3.7 and 3.8 for SNCD0.67 and SNCD1.0. We briefly investigate these regression equations and test of hypothesis next.

We observe that there is no obvious relationship between uncorrected or biascorrected coverage probability based on $\left(S N C D_{05}, S N C D_{95}\right)$ and explanatory variables considered and confirms what we observe in Figure 3.7. On the other hand, multiple regression equations obtained for bias and uncorrected and bias-corrected coverage probabilities based on (SNCDL, SNCDU) are functions of some of the explanatory variables considered. These regression equations provide more information than what we gained from the Figures 3.5, 3.6. Estimates of the intercepts also show that on average, coverage probabilities (both uncorrected and bias-corrected) based on $\left(S N C D_{05}, S N C D_{95}\right)$ is closer to nominal than the other confirming what we observe in those corresponding figures. 


\begin{tabular}{|c|c|c|c|c|c|}
\hline & \multicolumn{5}{|c|}{ Model I } \\
\hline & $C c P b_{0595}$ & $c P b_{0595}$ & $C c P b_{L U}$ & $c P b_{L U}$ & Bias \\
\hline$\overline{\beta_{0}}$ & 0.827 & 0.848 & 0.764 & 0.744 & $\begin{array}{l}-0.119 \\
\end{array}$ \\
\hline$\beta_{1}$ & - & - & -0.3 & -0.3 & - \\
\hline$\beta_{2}$ & - & - & -0.2 & -0.2 & - \\
\hline$\beta_{3}$ & - & - & -0.1 & -0.1 & - \\
\hline$\beta_{4}$ & - & - & - & - & - \\
\hline$\beta_{5}$ & - & - & - & - & - \\
\hline$\alpha_{1}$ & - & 0.1 & -0.3 & -0.2 & 0.1 \\
\hline$\alpha_{2}$ & - & - & -0.1 & - & 0.1 \\
\hline$\alpha_{3}$ & - & - & 0.1 & 0.1 & 0.1 \\
\hline$\alpha_{4}$ & - & - & 0.1 & 0.1 & 0.1 \\
\hline$\alpha_{5}$ & - & - & 0.1 & 0.1 & 0.1 \\
\hline$\overline{\theta_{1}}$ & - & - & 0.1 & 0.1 & - \\
\hline$\theta_{2}$ & - & - & - & - & - \\
\hline$\gamma_{1}$ & - & - & 0.1 & 0.1 & 0.1 \\
\hline$\gamma_{2}$ & - & - & 0.1 & 0.1 & - \\
\hline$\gamma_{3}$ & - & - & - & - & - \\
\hline$\gamma_{4}$ & - & - & - & - & - \\
\hline$\gamma_{5}$ & - & - & - & - & - \\
\hline
\end{tabular}

\begin{tabular}{|c||c|c|c|c|c||}
\hline \hline \multicolumn{1}{|c||}{} & \multicolumn{5}{c|}{ Model II } \\
\hline \hline$\beta_{0}$ & 0.856 & 0.861 & 0.845 & 0.822 & -0.072 \\
\hline$\beta_{1}$ & - & - & -0.2 & -0.3 & - \\
\hline$\beta_{2}$ & - & - & -0.1 & -0.1 & - \\
\hline$\beta_{3}$ & - & - & - & -0.1 & - \\
\hline$\beta_{4}$ & - & - & - & - & - \\
\hline$\beta_{5}$ & - & - & - & - & - \\
\hline \hline
\end{tabular}

\begin{tabular}{|c||c|c|c|c|c||}
\hline \hline$\alpha_{1}$ & - & - & -0.3 & -0.3 & 0.1 \\
\hline$\alpha_{2}$ & - & - & -0.1 & -0.1 & 0.1 \\
\hline$\alpha_{3}$ & - & - & - & -0.1 & - \\
\hline$\alpha_{4}$ & - & - & - & - & - \\
\hline$\alpha_{5}$ & - & - & - & - & - \\
\hline \hline$\theta_{1}$ & - & - & 0.1 & 0.1 & - \\
\hline$\theta_{2}$ & - & - & - & - & - \\
\hline \hline$\gamma_{1}$ & - & - & 0.1 & 0.1 & 0.1 \\
\hline$\gamma_{2}$ & - & - & - & 0.1 & - \\
\hline$\gamma_{3}$ & - & - & - & - & - \\
\hline$\gamma_{4}$ & - & - & - & - & - \\
\hline$\gamma_{5}$ & - & - & - & - & - \\
\hline \hline
\end{tabular}

Table 3.5: Multiple Regression Parameter Estimates (Parametric Bootstrap) when $p=0.67$ 


\begin{tabular}{|c|c|c|c|c|c|}
\hline & \multicolumn{5}{|c|}{ Model I } \\
\hline & $C c P b_{0595}$ & $c P b_{0595}$ & $\mathrm{CcPb}_{L U}$ & $c P b_{L U}$ & Bias \\
\hline$\beta_{0}$ & 0.827 & 0.831 & 0.746 & 0.739 & -0.08 \\
\hline$\beta_{1}$ & - & - & -0.3 & -0.2 & -0.1 \\
\hline$\beta_{2}$ & - & - & -0.2 & -0.2 & -0.1 \\
\hline$\beta_{3}$ & - & - & -0.1 & -0.1 & - \\
\hline$\beta_{4}$ & - & - & - & - & -0.1 \\
\hline$\beta_{5}$ & - & - & - & - & - \\
\hline$\alpha_{1}$ & - & 0.1 & -0.2 & 0.2 & 0.2 \\
\hline$\alpha_{2}$ & - & - & - & 0.1 & 0.1 \\
\hline$\alpha_{3}$ & 0.1 & 0.1 & 0.2 & 0.5 & 0.1 \\
\hline$\alpha_{4}$ & - & - & 0.1 & 0.1 & 0.1 \\
\hline$\alpha_{5}$ & - & - & 0.1 & 0.1 & 0.1 \\
\hline$\theta_{1}$ & - & - & 0.1 & 0.1 & - \\
\hline$\theta_{2}$ & - & - & - & - & - \\
\hline$\gamma_{1}$ & - & - & 0.2 & 0.2 & 0.1 \\
\hline$\gamma_{2}$ & - & - & 0.1 & 0.1 & - \\
\hline$\gamma_{3}$ & - & - & - & - & - \\
\hline$\gamma_{4}$ & - & - & - & - & - \\
\hline \multirow[t]{2}{*}{$\gamma_{5}$} & - & - & - & - & - \\
\hline & \multicolumn{5}{|c|}{ Model II } \\
\hline $\bar{\beta}_{0}$ & 0.856 & 0.860 & 0.788 & 0.788 & -0.026 \\
\hline$\beta_{1}$ & - & - & -0.2 & -0.2 & - \\
\hline$\beta_{2}$ & - & - & -0.1 & -0.1 & - \\
\hline$\beta_{3}$ & - & - & - & - & - \\
\hline$\beta_{4}$ & - & - & - & - & - \\
\hline$\beta_{5}$ & - & - & - & - & - \\
\hline$\alpha_{1}$ & - & - & -0.2 & -0.2 & $\overline{0.1}$ \\
\hline$\alpha_{2}$ & - & - & - & - & - \\
\hline$\alpha_{3}$ & - & - & 0.1 & 0.1 & - \\
\hline$\alpha_{4}$ & - & - & - & - & - \\
\hline$\alpha_{5}$ & - & - & - & - & - \\
\hline$\theta_{1}$ & - & - & 0.1 & 0.1 & - \\
\hline$\theta_{2}$ & - & - & - & - & - \\
\hline$\gamma_{1}$ & - & - & 0.1 & 0.1 & 0.1 \\
\hline$\gamma_{2}$ & - & - & 0.1 & 0.1 & - \\
\hline$\gamma_{3}$ & - & - & - & - & - \\
\hline$\gamma_{4}$ & - & - & - & - & - \\
\hline$\gamma_{5}$ & - & - & - & - & - \\
\hline
\end{tabular}

Table 3.6: Multiple Regression Parameter Estimates (Parametric Bootstrap) when $p=1.0$ 
Based on the p-values, we reject the test of hypothesis that the intercept is 0.9 for any of the coverage probabilities. Both the parameter estimates for multiple regression equations and p-values for the test of hypothesis for the bias confirm that there is no significant bias in the parametric bootstrap approach. Our conclusion for these response variables is that multiple regression equation provides little more insight into this relationship than the Figures 3.5, 3.6 and 3.7 provide.

\begin{tabular}{|c||c|c|}
\hline P.value & Model1 & Model2 \\
\hline \hline$C c P b_{0595}$ & $1.03 \mathrm{e}-9$ & 0.00 \\
\hline$c P b_{0595}$ & $3.6 \mathrm{e}-5$ & 0.00 \\
\hline$c P b_{L U}$ & $5.2 \mathrm{e}-4$ & $1.5 \mathrm{e}-3$ \\
\hline$c P b_{L U}$ & $3.6 \mathrm{e}-5$ & $1.9 \mathrm{e}-5$ \\
\hline Bias $_{S N C D}$ & $7.7 \mathrm{e}-15$ & 0.00 \\
\hline \hline
\end{tabular}

Table 3.7: Parametric Boostrap Approach P-values for $p=0.67$

\begin{tabular}{|c||c|c|}
\hline P.value & Model1 & Model2 \\
\hline \hline$C c P b_{0595}$ & $8.3 \mathrm{e}-7$ & 0.00 \\
\hline$c P b_{0595}$ & $7.7 \mathrm{e}-11$ & 0.00 \\
\hline$C c P b_{L U}$ & $3.2 \mathrm{e}-4$ & $5.3 \mathrm{e}-8$ \\
\hline$c P b_{L U}$ & $1.6 \mathrm{e}-4$ & $5.6 \mathrm{e}-8$ \\
\hline Bias $_{S N C D}$ & $1.6 \mathrm{e}-4$ & $1.0 \mathrm{e}-4$ \\
\hline \hline
\end{tabular}

Table 3.8: Parametric Boostrap Approach P-values for $p=1.0$

\section{Non-parametric Bootstrap Approach Results}

We present the parameter estimates for multiple regression equations derived for bias, uncorrected and bias-corrected probabilities based on (SNCDL, SNCDU) and $\left(S N C D_{05}, S N C D_{95}\right)$ in Tables 3.9 and 3.10 ; and p-values for the appropriate hypothesis test on the intercept of these multiple regression equations in Tables 3.11 and 3.12 for SNCD0.67 and SNCD1.0. 


\begin{tabular}{|c|c|c|c|c|c|}
\hline & \multicolumn{5}{|c|}{ Model I } \\
\hline & $C c P b_{0595}$ & $c P b_{0595}$ & $C c P b_{L U}$ & $c P b_{L U}$ & Bias \\
\hline$\beta_{0}$ & 0.800 & 0.795 & 0.737 & 0.714 & -0.013 \\
\hline$\beta_{1}$ & 0.01 & - & -0.3 & -0.2 & - \\
\hline$\beta_{2}$ & - & - & -0.2 & -0.2 & - \\
\hline$\beta_{3}$ & - & - & -0.1 & - & - \\
\hline$\beta_{4}$ & - & - & - & - & - \\
\hline$\beta_{5}$ & - & - & - & - & - \\
\hline$\alpha_{1}$ & 0.1 & 0.1 & -0.2 & -0.2 & - \\
\hline$\alpha_{2}$ & - & 0.1 & $0.1-$ & - & - \\
\hline$\alpha_{3}$ & 0.1 & 0.1 & 0.1 & 0.1 & - \\
\hline$\alpha_{4}$ & 0.1 & 0.1 & 0.1 & 0.1 & - \\
\hline$\alpha_{5}$ & 0.1 & 0.1 & 0.1 & 0.1 & - \\
\hline$\theta_{1}$ & - & - & 0.1 & 0.1 & - \\
\hline$\theta_{2}$ & - & - & - & - & - \\
\hline$\gamma_{1}$ & - & - & 0.1 & 0.1 & 0.1 \\
\hline$\gamma_{2}$ & - & - & 0.1 & 0.1 & 0.1 \\
\hline$\gamma_{3}$ & - & - & - & - & 0.1 \\
\hline$\gamma_{4}$ & - & - & - & - & - \\
\hline \multirow[t]{2}{*}{$\gamma_{5}$} & - & - & - & - & - \\
\hline & \multicolumn{5}{|c|}{ Model II } \\
\hline $\bar{\beta}_{0}$ & 0.842 & 0.844 & 0.824 & 0.811 & 0.000 \\
\hline$\beta_{1}$ & - & - & -0.2 & -0.2 & - \\
\hline$\beta_{2}$ & - & - & -0.2 & -0.2 & - \\
\hline$\beta_{3}$ & - & - & - & - & - \\
\hline$\beta_{4}$ & - & - & - & - & - \\
\hline$\beta_{5}$ & - & - & - & - & 0.1 \\
\hline$\alpha_{1}$ & - & 0.1 & -0.3 & -0.3 & - \\
\hline$\alpha_{2}$ & - & - & -0.1 & -0.1 & - \\
\hline$\alpha_{3}$ & - & - & - & - & - \\
\hline$\alpha_{4}$ & - & - & - & - & - \\
\hline$\alpha_{5}$ & - & 0.1 & - & - & - \\
\hline$\theta_{1}$ & - & - & 0.1 & 0.1 & - \\
\hline$\theta_{2}$ & - & - & - & - & - \\
\hline$\gamma_{1}$ & - & - & 0.1 & 0.1 & 0.1 \\
\hline$\gamma_{2}$ & - & - & 0.1 & 0.1 & - \\
\hline$\gamma_{3}$ & - & - & - & - & - \\
\hline$\gamma_{4}$ & - & - & - & - & - \\
\hline$\gamma_{5}$ & - & - & - & - & - \\
\hline
\end{tabular}

Table 3.9: Multiple Regression Parameter Estimates (Non-parametric Bootstrap) when $p=0.67$ 


\begin{tabular}{|c|c|c|c|c|c|}
\hline & \multicolumn{5}{|c|}{ Model I } \\
\hline & $C c P b_{0595}$ & $c P b_{0595}$ & $C c P b_{L U}$ & $c P b_{L U}$ & Bias \\
\hline$\beta_{0}$ & 0.857 & 0.863 & 0.771 & 0.753 & -0.062 \\
\hline$\beta_{1}$ & - & - & -0.2 & -0.2 & -0.1 \\
\hline$\beta_{2}$ & - & - & -0.2 & -0.2 & -0.1 \\
\hline$\beta_{3}$ & - & - & -0.1 & - & -0.1 \\
\hline$\beta_{4}$ & - & - & - & - & -0.1 \\
\hline$\beta_{5}$ & - & - & - & - & -0.1 \\
\hline$\alpha_{1}$ & - & - & -0.2 & -0.2 & 0.2 \\
\hline$\alpha_{2}$ & - & - & - & - & 0.2 \\
\hline$\alpha_{3}$ & - & - & 0.1 & 0.1 & 0.1 \\
\hline$\alpha_{4}$ & - & - & - & - & 0.1 \\
\hline$\alpha_{5}$ & - & - & - & - & 0.2 \\
\hline$\theta_{1}$ & - & - & 0.1 & 0.1 & - \\
\hline$\theta_{2}$ & - & - & - & - & - \\
\hline$\gamma_{1}$ & - & - & 0.2 & 0.2 & 0.1 \\
\hline$\gamma_{2}$ & - & - & 0.1 & 0.1 & 0.1 \\
\hline$\gamma_{3}$ & - & - & 0.1 & - & - \\
\hline$\gamma_{4}$ & - & - & - & - & - \\
\hline \multirow[t]{2}{*}{$\gamma_{5}$} & - & - & - & - & - \\
\hline & \multicolumn{5}{|c|}{ Model II } \\
\hline $\bar{\beta}_{0}$ & 0.858 & 0.865 & 0784 & 0.782 & 0.000 \\
\hline$\beta_{1}$ & - & - & -0.2 & -0.2 & - \\
\hline$\beta_{2}$ & - & - & -0.2 & -0.2 & - \\
\hline$\beta_{3}$ & - & - & - & - & - \\
\hline$\beta_{4}$ & - & - & - & - & - \\
\hline$\beta_{5}$ & - & - & - & - & - \\
\hline$\alpha_{1}$ & - & - & -0.2 & -0.2 & 0.1 \\
\hline$\alpha_{2}$ & - & - & - & - & - \\
\hline$\alpha_{3}$ & - & - & 0.1 & 0.1 & - \\
\hline$\alpha_{4}$ & - & - & - & - & - \\
\hline$\alpha_{5}$ & - & - & - & - & - \\
\hline$\theta_{1}$ & - & - & 0.1 & 0.1 & - \\
\hline$\theta_{2}$ & - & - & - & - & - \\
\hline$\gamma_{1}$ & - & - & 0.1 & 0.1 & 0.1 \\
\hline$\gamma_{2}$ & - & - & 0.1 & 0.1 & - \\
\hline$\gamma_{3}$ & - & - & - & - & - \\
\hline$\gamma_{4}$ & - & - & - & - & - \\
\hline$\gamma_{5}$ & - & - & - & - & - \\
\hline
\end{tabular}

Table 3.10: Multiple Regression Parameter Estimates (Non-parametric Bootstrap) when $p=1.0$ 
Overall, we see similar results that we observe when parametric bootstrap approach is applied. There is no obvious relationship between these five response variables and their corresponding explanatory variables, other than that estimates of the intercepts for coverage probabilities based on $\left(S N C D_{05}, S N C D_{05}\right)$ are closer to the nominal than that based on (SNCDL, SNCDU). We see no significant improvement when bias-correction is done. These multiple regression equations provide little more insight into the relationship than the Figures 3.8, 3.9 and 3.10 provid.

\begin{tabular}{|c||c|c|}
\hline P.value & Model1 & Model2 \\
\hline \hline$C c P b_{0595}$ & $1.5 \mathrm{e}-13$ & 0.00 \\
\hline$c P b_{0595}$ & $1.3 \mathrm{e}-9$ & 0.00 \\
\hline$C c P b_{L U}$ & $6.2 \mathrm{e}-5$ & $3.5 \mathrm{e}-5$ \\
\hline$c P b_{L U}$ & $5.5 \mathrm{e}-6$ & $1.9 \mathrm{e}-6$ \\
\hline$B i a s_{S N C D}$ & 0.631 & 0.783 \\
\hline \hline
\end{tabular}

Table 3.11: Non-parametric Boostrap Approach P-values for $p=0.67$

\begin{tabular}{|c||c|c|}
\hline Test & Model1 & Model2 \\
\hline \hline$C c P b_{0595}$ & $2.5 \mathrm{e}-7$ & 0.00 \\
\hline$c P b_{0595}$ & 0.012 & $1.3 \mathrm{e}-11$ \\
\hline$C c P b_{L U}$ & $2.8 \mathrm{e}-3$ & $3.6 \mathrm{e}-8$ \\
\hline$c P b_{L U}$ & $6.9 \mathrm{e}-4$ & $2.2 \mathrm{e}-8$ \\
\hline$B i a s_{S N C D}$ & 0.201 & 0.872 \\
\hline \hline
\end{tabular}

Table 3.12: Non-parametric Boostrap Approach P-values for $p=1.0$

Although uncorrected coverage probability based on $\left(S N C D_{05}, S N C D_{95}\right)$ performed better for both parametric and non-parametric bootstrap approaches than the corresponding analytical approach probabilities, bias-corrected coverage probability failed to do so for these bootstrap approaches. Hence, We find no use for these multiple regression equations obtained using these bootstrap approaches. We choose to further investigate the analytical bias observed as the bias-correction significantly improves the coverage probabilities based on both (SNCDL, SNCDU) and $\left(S N C D_{05}, S N C D_{95}\right)$. 


\subsubsection{Quantitative Explanatory Variables}

Multiple regression equation for bias in signal-to-noise crossover dose estimates as a function of $\kappa, \eta$, number of dose levels, number of trials per dose and maximum dose as quantitative variables is given by

$$
y=\beta_{0}+\beta_{1} \kappa+\beta_{2} \eta+\beta_{3} k+\beta_{4} n+\beta_{5} d_{k}+\cdots+\epsilon
$$

where $\cdots=$ all possible interaction of $\kappa, \eta, \mathrm{k}, \mathrm{n}$ and $d_{k}$.

This multiple regression model is fitted for analytically estimated bias which is significant compared to either the parametric or non-parametric bootstrap estimated biases. This model is fitted for analytically estimated bias as a quantitative response variable and $\kappa, \eta$, number of trials per dose, number of dose levels and maximum dose as quantitative explanatory variables. Appropriate reduced models are obtained using partial F-test for both the SNCD1.0 and SNCD0.67 estimates.

This analytical bias is estimated for $(\kappa / \mathrm{mxd}, \eta)=((0.5,3),(0.625,1),(0.875$, $2),(1.0,5))$ combinations with maximum doses of 16 and number of doses of five, in addition to all possible combinations of sixteen different $(\kappa / \mathrm{mxd}, \eta)$ combinations, three different number of doses and six different number of trials per dose with maximum dose $\left(d_{k}\right)$ of 8 . Multiple regression model in 3.46 is fitted on these data and appropriate reduced model is obtained.

Our initial analysis show that $R^{2}$ for reduced multiple regression equation is around 0.5 for both SNCD0.67 and SNCD1.0 (first two columns of Table 3.13) and the model assumptions are not satisfied either. Data points causing this discrepancy are removed (i.e data obtained for $\kappa / d_{k} \geq 1.25$ are excluded). The model is fitted for subset of the original data. Multiple regression equation with $R^{2}$ above 0.8 is obtained for both SNCD0.67 and SNCD1.0. The model assumptions are satisfied as well (Figures 3.11 and 3.12). Multiple regression parameter estimates for SNCD1.0 and SNCD0.67 are presented in the last two column of Table 3.13 which includes 


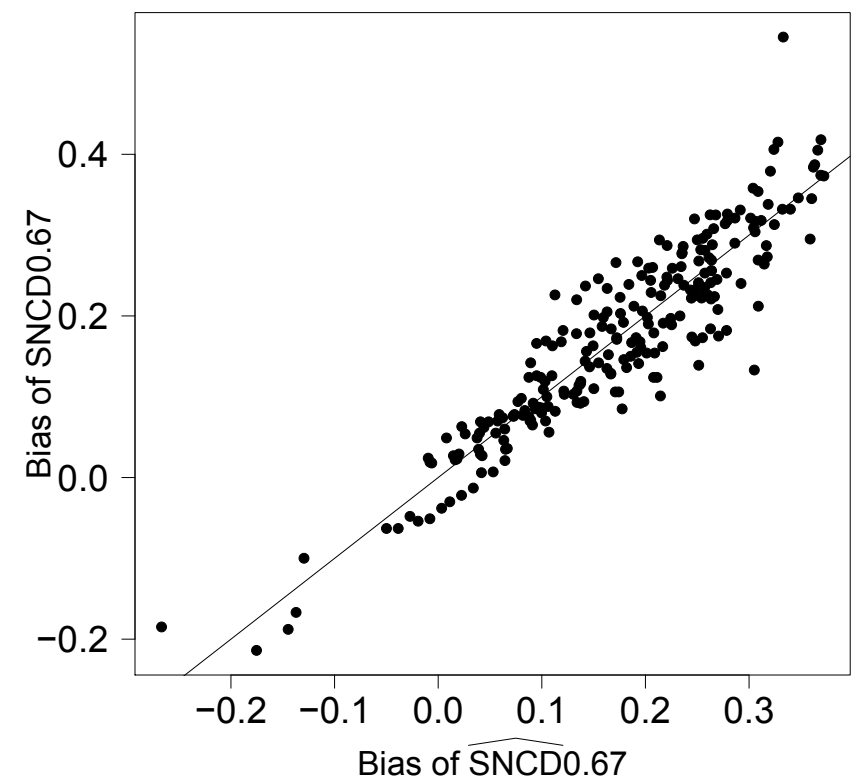

Figure 3.11: Actual vs Fitted Bias of SNCD0.67 for Subset of Data

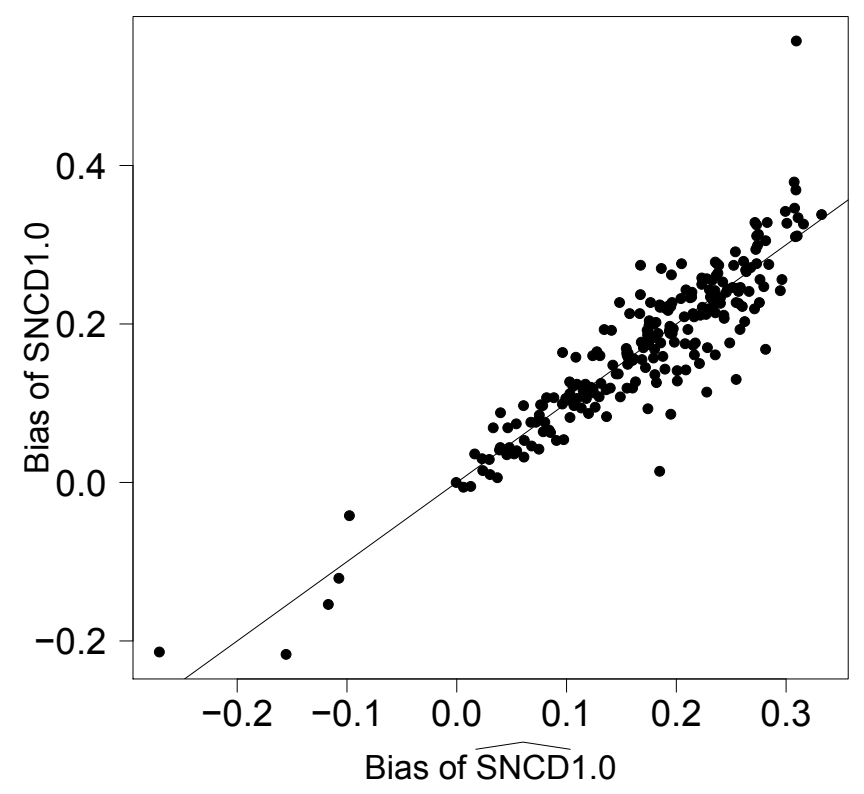

Figure 3.12: Actual vs Fitted Bias of SNCD1.0 for Subset of Data 
estimates and the significance the estimates for SNCD0.67 and SNCD1.0.

\begin{tabular}{|c|c|c|c|c|}
\hline \multirow[b]{2}{*}{ Bias } & \multicolumn{2}{|c|}{ Est (all data) } & \multicolumn{2}{|c|}{ Est (subset data) } \\
\hline & SNCD1.0(sig) & SNCD0.67(sig) & SNCD1.0(sig) & SNCD0.67(sig) \\
\hline intercept & $-1.522^{* * *}$ & $-1.716^{* * *}$ & $-1.084^{* * *}$ & $-1.093^{* * *}$ \\
\hline$\kappa$ & $2.7 e-1^{* * *}$ & $3.0 e-1^{* * *}$ & $2.2 e-1^{* * *}$ & $2.1 e-1^{* * *}$ \\
\hline$\eta$ & $0.8 e-1^{* * *}$ & $8.6 e-1^{* * *}$ & $4.5 e-1^{* * *}$ & $4.6 e-1^{* * *}$ \\
\hline$k$ & $8.7 e-2^{* * *}$ & $1.0 e-1^{* * *}$ & $2.0 e-2^{* *}$ & $2.6 e-2^{* * *}$ \\
\hline$n$ & $-4.9 e-4^{* * *}$ & $-3.8 e-4^{* * *}$ & $1.7 \mathrm{e}-4$ & $3.2 e-4^{\bullet}$ \\
\hline$d_{k}$ & $1.2 e-1^{* * *}$ & $1.2 e-1^{* * *}$ & $1.1 e-1^{* * *}$ & $9.9 e-2^{* * *}$ \\
\hline$\kappa * \eta$ & $-1.2 e-1^{* * *}$ & $-1.2 e-1^{* * *}$ & $-6.8 e-2^{* * *}$ & $-6.0 e-2^{* * *}$ \\
\hline$\kappa * k$ & $-1.3 e-2^{* * *}$ & $-1.5 e-2^{* * *}$ & $-4.3 e-3^{* * *}$ & $-3.0 e-3^{*}$ \\
\hline$\kappa * n$ & $2.8 e-5^{\bullet}$ & - & $-5.4 e-5^{*}$ & $-1.1 e-4^{* * *}$ \\
\hline$\kappa * d_{k}$ & $-1.8 e-2^{* * *}$ & $-1.9 e-2^{* *}$ & $-1.6 e-2^{* * *}$ & $-1.6 e-2^{* * *}$ \\
\hline$\eta * k$ & $-4.1 e-2^{* * *}$ & $-5.0 e-2^{* * *}$ & - & $-6.5 e-3^{* * *}$ \\
\hline$\eta * n$ & $6.8 e-5^{* *}$ & $7.1 e-5^{* *}$ & $-1.6 e-4^{*}$ & $-2.5 e-4^{* *}$ \\
\hline$\eta * d_{k}$ & $-6.4 e-2^{* * *}$ & $-6.5 e-2^{* * *}$ & $-5.3 e-2^{* * *}$ & $-4.9 e-2^{* * *}$ \\
\hline$n * d_{k}$ & - & - & $-1.8 e-5^{*}$ & $\begin{array}{ll}- \\
-\end{array}$ \\
\hline$\kappa * \eta * n$ & - & - & $3.5 e-5^{* * *}$ & $4.8 e-5^{* * *}$ \\
\hline$\kappa * \eta * d_{k}$ & $8.9 e-3^{* * *}$ & $9.0 e-3^{* * *}$ & $7.3 e-3^{* * *}$ & $6.7 e-3^{* * *}$ \\
\hline$\kappa * \eta * k$ & $5.7 e-3^{* * *}$ & $6.6 e-3^{* * *}$ & - & - \\
\hline MSE & $\overline{6.7 \mathrm{e}-3}$ & $\overline{9.7 \mathrm{e}-3}$ & $\overline{1.8 \mathrm{e}-3}$ & $2.2 \mathrm{e}-3$ \\
\hline $\mathrm{SE}$ & 0.082 & 0.099 & 0.042 & 0.047 \\
\hline$R^{2}$ & 0.567 & 0.525 & 0.823 & 0.855 \\
\hline
\end{tabular}

Table 3.13: Reduced Model for Biases of SNCD1.0 and SNCD0.67 with Quantitative Variables

We introduce four additional approaches to estimate the bias observed. These four approaches are compared with multiple regression approach. The best method among these five is to be used when analytical approach is applied to estimate the signal-to-noise crossover dose for our real datasets.

\subsubsection{Estimation and Assessment of Bias Correction}

We introduce four new approaches to estimate the bias realized in the SNCD or SNR estimates in addition to the multiple regression equation obtained to estimate the 
bias in SNCD. We choose to assess the estimate of bias using the confidence interval based on $\left(S N C D_{05}\right.$ and $\left.S N C D_{95}\right)$ as this confidence interval showed improved results than that based on (SNCDL, SNCDU) in the analytical approach.

(1) Bias of each simulation is estimated by subtracting estimated SNCD for the specific simulation from the average of SNCD over the 500 bootstrap done in that simulation (referred btsncd).

(2) Multiple regression equations (Last two columns of Table 3.13) derived in chapter 3.5.2 to estimate the bias observed in the SNCD. The bias is estimated using these multiple regression equations (referred mltRg).

(3) For each of the simulation, expectation of $\log$ of $\widehat{S N R}\left(d_{i}\right)$ is estimated using Equation 3.24. Bias of $\log \widehat{S N R}\left(d_{i}\right)$ is obtained by subtracting the log of $\widehat{S N R}\left(d_{i}\right)$ in a simulation from expectation of $\log$ of $\widehat{S N R}\left(d_{i}\right)$. Bias correction is done on $\log$ of $\widehat{S N R}\left(d_{i}\right)$, and the bias corrected SNCD, $S N C D_{05}$ and $S N C D_{95}$ are calculated (referred scdodr).

(4) Bias of $\widehat{S N R}\left(d_{i}\right)$ is estimated by obtaining the difference between $\widehat{S N R}\left(d_{i}\right)$ in a simulation and average over 500 bootstrap estimated $\widehat{S N R}\left(d_{i}\right)$ of that simulation. Bias correction is done on $\widehat{S N R}\left(d_{i}\right)$ and the corresponding SNCD, $S N C D_{05}$ and $S N C D_{95}$ are obtained (referred btsnr).

(5) Bias is estimated by subtracting log of $\widehat{S N R}\left(d_{i}\right)$ in a simulation from average of $\log$ of $\widehat{S N R}\left(d_{i}\right)$ of 500 bootstraps in that simulation. Bias correction is done on the $\log$ of $\widehat{S N R}\left(d_{i}\right)$ and the corresponding SNCD, SNCD 05 and $S N C D_{95}$ are estimated (referred btlnsnr).

Five-hundred bootstraps (in page48 of chapter 3.1.1) are done within each of 1000 simulation for each of four different hill model parameter combination with four doses and practically applicable 50 trials per dose. Bias of SNCD is estimated using 
these five different methods with the purpose of selecting the best method to estimate the bias. Using the bias corrected $S N C D,\left(S N C D_{05}, S N C D_{95}\right)$ for $\mathrm{p}=2 / 3$ and 1 , the corresponding coverage probability of SNCD1.0 and SNCD0.67 are calculated.

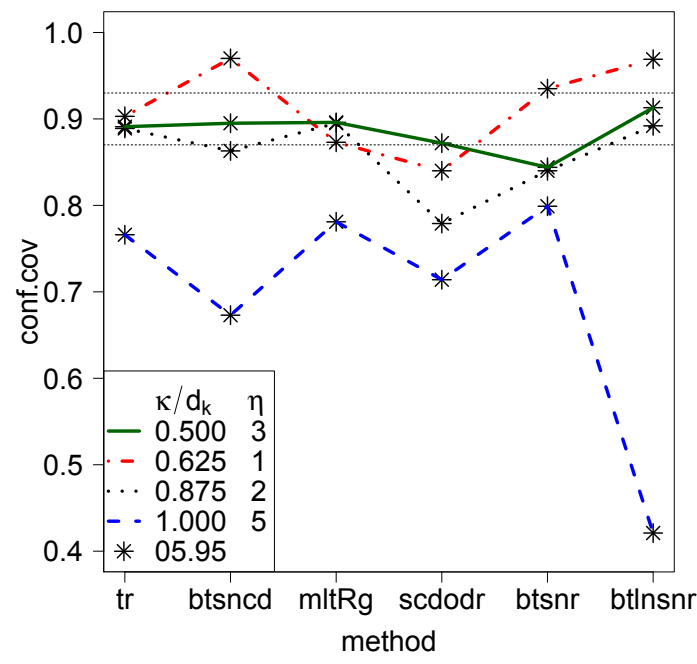

(a) Est.Bias of SNCD0.67

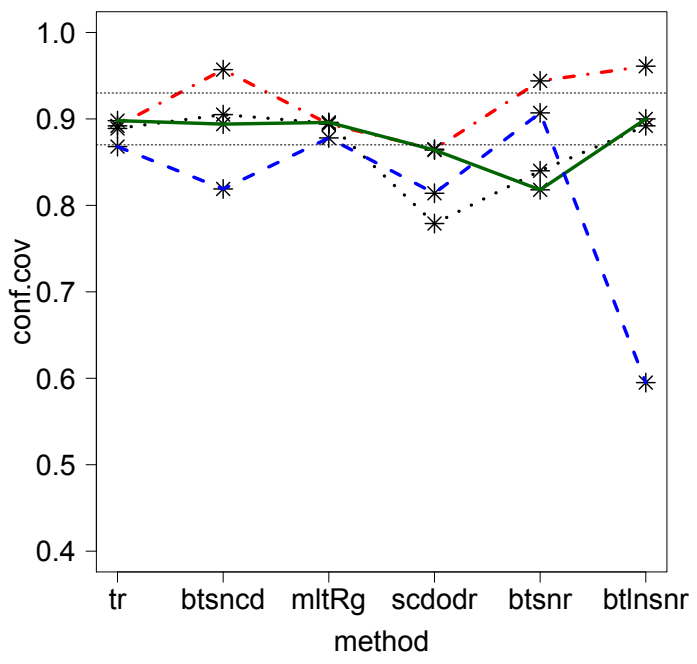

(b) Est.Bias of SNCD1.0

Figure 3.13: Coverage Probabilities for Five different Bias-corrected Estimates of SNCD0.67 and SNCD1.0

The bias-corrected coverage probability obtained using these five methods is compared with that of true bias correction. Figure 3.13 shows the plots of coverage for the four different selected hill model parameter combinations. The coverage probability obtained by correcting the bias using multiple regression approach is similar to true bias corrected coverage for both SNCD0.67 and SNCD1.0. Therefore, we choose the multiple regression equation to estimate the bias when we use the analytical approach to estimate the signal-to-noise crossover dose on our real datasets.

\subsection{Summary and Conclusion}

We introduce three different methods to estimate the SNCD. These methods are analytical, parametric bootstrap and non-parametric bootstrap method. Further, the goodness of the estimates of the SNCD is assessed using two different confidence 
intervals which are (SNCDL, SNCDU) and $\left(S N C D_{05}, S N C D_{95}\right)$. Our simulation studies show that estimates obtained using analytical approach with bias correction give the best estimates. We introduce and compare five different approaches to estimate the bias realized in the SNCD estimates, and identify the best approach to estimate the bias.

Using simulation studies, we estimate the point and confidence interval estimates based on (SNCDL, SNCDU) and $\left(S N C D_{05}, S N C D_{95}\right)$ for all possible combination of four different shapes of Hill model curves, three different dose levels and six different trial per dose. The shapes of Hill curves include S-shape, convex, concave with initial shape greater than zero and concave with initial slope zero; numbers of dose levels include four, six and eight; and numbers of trials per dose include 50, 100, 200, 300, 400 and 500. We calculate bias, the uncorrected and bias-corrected coverage probabilities based on (SNCDL, SNCDU) and $\left(S N C D_{05}, S N C D_{95}\right)$ for these estimates by applying the methods developed in this chapter.

We calculate the same measures - bias, uncorrected and bias-corrected coverage probabilities based on (SNCDL, SNCDU) and $\left(S N C D_{05}, S N C D_{95}\right)$ - using parametric and non-parametric bootstrap methods.

First, we investigate the relationship between each of these five response variables and the shape, number of dose levels and number of trials per dose. For that, we carry out a multiple regression analysis for bias, uncorrected and bias-corrected coverage probabilities based on both (SNCDL, SNCDU) and $\left(S N C D_{05}, S N C D_{95}\right)$ as response variables and the Hill model parameters $\kappa$ and $\eta$, the number of dose levels and the numbers of trials per dose as qualitative explanatory variables. The corresponding multiple regression equations and the test of hypothesis performed on the intercepts of these equations confirm the following. Bias-corrected coverage based on $\left(S N C D_{05}, S N C D_{95}\right)$ reported excellent results and there is a bias in the estimates. We repeat this procedure for the same five response variables calculated 
using parametric and non-parametric approach. Our results show that there is no noticeable relationship between these response variables and explanatory variables in the bootstrap approaches. Bias-corrected analytical approach reports the best results. Therefore, we obtain a multiple regression to estimate the bias as a function of the Hill model parameters, $\kappa$ and $\eta$; number of dose levels and number of trials per dose as quantitative variables for analytical approach results. We introduce four new approaches to estimate this bias and compare with the multiple regression equation results. The results show that bias-correction using multiple regression gives the best results. This multiple regression equation to estimate the bias is used to correct the bias in the estimates of the SNCD in the real data results. 


\section{BIBLIOGRAPHY}

[1] Allen.B.C., Kavlock.R.J., Kimmel.C.A. and Faustman.E.M., (1994a) Dose-response assessment for developmental toxicity II. Comparison of generic benchmark dose estimates with no observed adverse effect levels, Fundamental of Applied Toxicology 23:487-495

[2] Auton.T.R. (1994) Calculation of Benchmark Doses from Teratology Data, Regulatory Toxicology and Pharmacology 19, 152-167 (1994).

[3] Barnes.D.G. and Dourson.M. (1988) Reference dose (RfD): description and use in health risk assessments, Regulatory Toxicology and Pharmacology 8:471-486

[4] Barnes.D.G. and Dourson.M. (1988) Reference dose (RfD): description and use in health risk assessments, Regulatory Toxicology and Pharmacology 8:471-486

[5] Bokkers.B.G.H., and Slob.W. (2007) Deriving a Data-based interspecies Assessment Factor Using the NOAEL and the Benchmark Dose Approach, Critical Reviews in Toxicology 37:355-373.

[6] Dourson.M., Felter.S. and Robinson.D. Evolution of science-based uncertainity factors in noncancer risk assessment, Regul Toxicol Pharmacol 24:108-120

[7] Evans.J.S., Graham.J.D., Gray.G.M., and Sielken, Jr.R.L (1994) A Distributional Approach to Characterizing Low-Dose Cancer Risk, Risk Analysis, Vol.14, No.1, 1994 
[8] EFSA 2005 Opinion of the Scientific Committee on a request from EFSA related to a harmonised approach for risk assessment of substances which are both genotoxic and carcinogenic, The EFSA Journal 282:1-31

[9] EFSA 2009a Guidance of the Scientific Committee on a request from EFSA on the use of the benchmark dose approach in risk assessment, The EFSA Journal 1150:1-72

[10] Evans.J.S., Graham.J.D., Gray.G.M., and Sielken, Jr.R.L (1994) A Distributional Approach to Characterizing Low-Dose Cancer Risk, Risk Analysis, Vol.14, No.1, 1994

[11] Foster.P.M.D., and Auton.T.R. (1995) Application of benchmark dose risk assessment methodology to developmental toxicity: an industrial view, Toxicology Letters 82.83 (1995) 555-559

[12] Fowles.J.R., Alexeeff.G.V. and Dodge.D., (1999) The use of benchmark dose methodology with acute inhalation lethality data, Regulatory Toxicological Pharmacology 29:151-160

[13] Gart.J.J., Krewski.D., Lee.P.N., Tarone.R.E. and Wahrendorf.J. (1986) Statistical Methods in Cancer Research Volumne III, International Agency fro Research on Cancer, WHO, (1986)

[14] Gaylor.D. (1992) Incidence of Developmental Defects at the No Observed Adverse Effect Level (NOAEL), Regulatory Toxicology and Pharmacology $15,151-160(1992)$

[15] Gaylor.D., Ryan.L., Krewski.D., and Zhus.Y., (1998) Procedures for Calculating Benchmark Doses for Health Risk Assessment, Regulatory Toxicology and Pharmacology 28, 150-164 (1998) 
[16] Izadi.H., Grundy.J.E., and Bose.R. (2012) Evaluation of the Benchmark Dose for Point of Departure Determination for a Variety of Chemical Classes in Applied Regulatory Settings, Risk Analysis, Vol.32, No. 5 (2012)

[17] Kavlock.R.J., Schmid.J.E., and Setzer,Jr.R.W. (1996) A Simulation Study of the Influence of Study Design on the Estimation of Benchmark Doses for Developmental Toxicity, Risk Analysis Vol.16, No.3, (1996)

[18] Kimmel.C.A., Gaylor.D.W. (1988) Issues in Qualitative and Quantitative Risk Analysis for Developmental Toxicology, Risk Analysis, Vol.8, No.1, (1988)

[19] Krewski.D., Bickis.M., Kovar.J., and Arnold.D.L. (1986) Optimal Experimental Designs for Low Dose Extrapolation I, The case of zero background, Utilitas Mathematica 29(1986), pp.245-262

[20] Krewski.D, Hogan.V, Turner.M.C, Zeman.P.L, McDowell.I, Edwards.N and Losos.J. (2007) An integrated framework for risk management and population health. Human and Ecological Risk Assessment 13:12881312

[21] Lehman,A.J., and Fitzhugh,O.G., (1954) 100-fold margin of safety, Assoc.Food Drug Off. U.S.Q. Bull. 18:33-35.

[22] Leisenring.W and Ryan.L. (1992) Statistical Properties of the NOAEL, Regulatory Toxicology and Pharmacology 15:161-171

[23] McCelellan.R.O., (1999) A historical Overview and alternative paths forward, Inhalation Toxicology 11:477-518 
[24] Moerbeek.M., Piersma.A.H., and Slob.W. (2004) A Comparison of Three Methods for Calculating Confidence Intervals for the Benchmark Dose, Risk Analysis, Vol. 24, No. 1, 2004

[25] Pease.W., Vandenberg.J., and Hooper.K., (1991) Comparing Alternative Approaches to Establishing Regulatory Levels for Reproductive Toxicants: DBCP as a Case Study, Environmental Health Perspectives, Vol.91 pp, 141-155

[26] Sand.S., Victorin.K. and Filipsson.A.F (2008) The current state of knowledge on the use of the benchmakr dose concept in risk assessment, Journal of Applied Toxicology 28:405-421

[27] Sand.S., Portier.C.J., and Krewski.D.,(2011) A Signal-to-Noise Crossover Dose as the Point of Departure for Health Risk Assessment, Environ Health Perspect 119:1766-1774

[28] Wheeler.M.W., and Bailer.A.J., (2013) An empirical comparison of lowdose extrapolation from points of departure compared to extrapolations based upon methods that account fro model uncertainity, Regulatory Toxicology and Pharmacology 67 (2013) 75-82

[29] William.P., Vandenberg.J., and Hooper.K. (1991) Comparing Alternative Approaches to Establishing Regulatory Levels for Reproductive Toxicants: DBCP As a Case Study, Environ Health Perspect Vol.91, pp.141-155, 1991

[30] Zhu.Y., Wang.T., and Jelsovsky.J.Z.H. (2007) Bootstrap Estimation of Benchmark Doses and Confidence Limits with Clustered Quantal Data, Risk Analysis, Vol. 27, No. 2, 2007 


\section{EMPIRICAL COMPARISON OF THE SIGNAL-TO-NOISE CROSSOVER DOSE AND THE BENCHMARK DOSE}

\subsection{Introduction}

A central problem in population health risk assessment is the establishment of a point of departure $(\mathrm{PoD})$ on the dose-response curve for toxic environmental agents (Krewski et al., 2014). PoDs serve as a basis for the establishment of human exposure guidelines, following the application of appropriate adjustment factors taking into account to reflect interspecies differences when extrapolation from animals to humans, inter-individual variability within the human population, and various aspects of data quality and completeness. Historically, Lehman and Fitzhugh (1954) first introduced the NOAEL (No-Observed-Adverse-Effect-Level) and LOAEL (LowestObserved-Adverse-Effect-Level) as a PoD for establishing human exposure guidelines. While this measure has been used for many years in dose-response assessment, its limitations have also been recognized. The NOAEL, for example, is restricted to being one of the experimental doses, and increases, rather than decreases, with decreasing sample size.

These limitations may be circumvented by the use of the benchmark dose. Benchmark dose (BMD), introduced by Crump (1984), is the dose corresponding to a specified increase in risk (usually in the range of $1-10 \%$, and referred to as the benchmark response or BMR) based on a fitted dose-response model. In their opinion on BMD, EFSA (European Food Safety Authority) points out that the BMR cannot be set at levels that are so low (or high) that estimation of the BMD requires extrapolation beyond the range of the experimental data, in which case the results

may become highly model dependent (EFSA 2009a). To overcome this limitation found in the BMD, signal to noise crossover dose (SNCD) is introduced recently. 
The signal to noise crossover dose (SNCD) introduced by Sand et al. (2011) provides an estimate of the lowest dose that can be derived as a PoD for risk assessment without resorting to low-dose extrapolation: this is accomplished by finding the point at which the biological signal is equal to the background noise, thereby defining the lower limit on the observable response range. The statistical procedures involved in estimating the SNCD are generally similar to those used to estimate the BMD. They differ, however, with respect to the definition of the PoD, which results in differences in the details estimating these two quantities. Sand et al. (2011) compared the SNCD (without confidence limits) to the BMDL (the lower confidence limit on the BMD) using over 800 sets of carcinogen bioassay data generated under the US National Toxicology Program. The work by Sand et al. (2011) did not include confidence limits on the SNCD. We developed both asymptotic and bootstrap confidence interval procedures for the SNCD and evaluated the performance of the new asymptotic and bootstrap confidence limit procedures using computer simulation, which served to validate the performance of these procedures in terms of coverage probability (Chapter 3). Previously in the literature, many different theoretical and empirical approaches have been used to estimate and to compare benchmark dose and NOAEL. Some of them are discussed herein.

Bioassay data from Teratology, Vol.1 (1968) through Vol.40(1990) was used by Gaylor (1992) [13] to compare risk at the NOAEL to the BMD with risk at the same order. As their analysis showed that the observed risk at the NOAEL exceeded $1 \%$ in about one-fourth of the cases, they concluded that the BMDL [4] would eliminate the higher risks and serve as a basis for establishing reference doses (RfDs). They also concluded that better experimental designs with larger sample sizes would result in tighter confidence limits on the BMD, thereby leading to larger RfD's than possibly underpowered experiments with smaller sample sizes. Faustman el. al. (1994) [7], [8] and [9] used a large database of 246 developmental toxicity experiments 
(Segment II-type studies) representing 1,825 different datasets to compare the use of the NOAEL and BMD approaches to developmental toxicity assessment, concluding that the theoretical basis and empirical findings of their investigation strongly support the use of the BMD approach for developmental toxicity RfD estimation. Acute lethality datasets was used by Fowles et. al. (1999)([11]) to compare BMDs at BMRs of 1, 5 and $10 \%$ incidences to NOAELs and LOAELS using log-normal probit and quantal Weibull models to fit to the experimental data. They concluded that the probit model repeatedly gave a better fit for the data compared with Weibull model resulting in improved goodness of fit and reduced mean width of the $95 \%$ confidence intervals.

Novel methods (Dourson et. al. 1985) [5] for determining an acceptable daily intake (ADI) was introduced to circumvent some of the limitations inherent in available approaches. These methods included a revised approach to estimating ADIs for partial-life time assessment using all toxicity data; and new methods for ADI estimation using quantal or continuous toxicity data. Though promising results were observed, conclusion is that more work is needed on these methods to be accepted as the status quo.

Using a historical database of teratology bioassays, Auton (1994) [2] evaluated the practical use of the benchmark dose by applying dose-response models to the data. They also expressed the view that as the BMD is based on the data from all dose levels of a study, it is preferable to NOAEL as a basis for risk assessment. Further, they reported that lower confidence limit on the dose giving $5 \%$ increase in incidence was seen as an appropriate definition of the BMD for most compounds examined in their analysis, except when the dose-response model does not adequately fit the data or when the estimated BMD falls outside the experimental range.

Comparing the BMD and NOAEL methods, Travis et al. (2005) [21] concluded that the NOAEL and BMD have complementary roles in risk assessment: the NOAEL 
provides a simple summary measure of adverse effects seen in toxicological studies, while the BMD is preferred for the interpretation of data from the key studies on which a human exposure guideline is based [21]. Methods for estimating the benchmark dose based on overdispersed data from developmental toxicity studies were compared by Fung.et.al (1998) [12]. These authors pointed out the importance of having optimal experimental designs and efficient risk estimation methods; they identified that NOAEL to provide a rough approximation to the BMD05 with average risk at the NOAEL ranging from 3 to $10 \%$ and concluded that the development of more biologically-based models is needed to better understand the nature of dose-response relationships for developmental toxicants.

The delta method and the bootstrap method were used to calculate (Zhu et. al. 2007) [22] the BMD and BMDL. Applying these methods to clustered binary data from developmental toxicity experiments illustrated that bootstrap BMDLs are smaller than the delta method BMDLs on average, hence quantifying risks more conservatively. The results showed that bootstrap BMDL quantifies the uncertainty of the true BMD more accurately than the delta method BMDL, as the bootstrap coverage probability is closer to nominal level than that of the delta method BMDL. The BMD and BMDL were found to be generally insensitive to model choices, provided that the models fit the data comparably well in the region of BMD.

Using data from a study on the developmental toxicity of BBP in the rat, three different methods are compared to calculate the confidence intervals for benchmark dose in risk analysis (Moerbeek et. al. 2004) [15]. This study looked at the performance of three such methods in fitting nonlinear dose-response models: the delta method, the likelihood-ratio method and the bootstrap method. Moerbeek et. al. (2004) reported that the bootstrap method and likelihood-ratio method gave similar results; the delta method, on the other hand, resulted in narrower intervals in some cases and appeared unreliable for nonlinear dose-response models. 
West et al. (2009) first assessed the suitability of a method that appealed to the large sample normality of parameter estimates, for use with small sample sizes; and proposed a new bootstrap technique as an alternative to the large sample methodology using simulation studies and examples from environmental toxicology. Based on their empirical results, the authors concluded that the coverage probability for the bootstrap procedure will often be close to nominal.

Bailer. et.al. (2005) [2] applied the multistage model to cancer bioassay data, and fitted different quantal models to kidney lesion data: a novel aspect of this work was the use of Bayesian model averaging (BMA) to obtain uncertainty limits on the BMD that reflect model uncertainty. Shao et al. (2014) discussed Bayesian model averaging as an approach to BMD estimation with continuous data that take account of the model uncertainty. They used a hybrid method proposed by Crump based on two strategies for implementing BMA, including both maximum likelihood estimation based and Markov Chain Monte Carlo based methods, to calculate model averaged BMD estimates from real continuous dose-response data. They chose dose-response models that are commonly used in Benchmark Dose Software (BMDS). Selection of models included are polynomial (quadratic and cubic), power, Hill, and exponential model with two different constant variance at all dose levels. They have concluded that BMA BMD estimates have higher reliability than estimates from the individual models with highest posterior weight in terms of higher BMDL and smaller 90th percentile intervals. The BMA BMD estimates were found to have smaller bias than BMDs selected using other criteria. However, it was recommended that further work be undertaken to validate this method.

Piegorsch et. al. (2014) [16] argue that a nonparametric approach can provide a useful alternative for BMD estimation when faced with parametric model uncertainty. They applied this approach in estimating BMDs with quantal-response data using isotonic regression, and explored the confidence coverage of this approach in small- 
sample properties via computer simulation. They emphasize that their nonparametric method requires both the number of doses and per dose sample sizes to grow large. Further, as their method is based on linearly interpolating the non-parametrically estimated risk function, if the dose-response pattern is highly concave and the number of doses is small, then they do not recommend this approach.

Sand et. al.(2011) [18] used the NTP carcinogen bioassay database to estimate PoDs and RfDs based on the SNCD and to compare the SNCD and SNCD-based RfD with PoDs and RfDs based on the NOAEL and BMD approaches. The purpose of this chapter is to extend the work of Sand et. al. (2011) to estimate PoDs based on BMD, BMDL, SNCD and SNCDL using theoretical approaches developed in the previous chapters applied to the same NTP database used by Sand et. al. (2011).

Building on our investigation of the properties of the BMD under the Hill model in Chapter 2, we developed a theoretical framework to estimate the SNCD and SNCDL in Chapter 3. Of the analytical, parametric and non-parametric bootstrap methods considered, the analytical methods combined with 500 bootstrap sample within each simulation gave better coverage with bias correction. Further, the biases associated with these estimation procedures are estimated using simulation studies for a wide range of Hill model parameter combinations and experimental designs with different numbers of doses and different dose allocations. A multiple regression equation is used to estimate the bias in the SNCD as a function of Hill model parameters, number of doses, sample size per dose, and the maximum dose. This equation is used to correct the bias observed in the SNCD calculated using the analytical methods described in Chapter 3.

BMDs and BMDLs are estimated for BMRs of $01 \%, 05 \%$ and $10 \%$, as this range of BMR is often a interest to risk regulators [10] [19]. Ratios of the BMDL to the BMD at BMRs of $01 \%, 05 \%$ and $10 \%$ are calculated, as are ratios of the SNCDL to the SNCD at SNRs of 1 and 2/3. Ratios of the BMD to SNCD (for all combinations 
of the BMR and SNR), and the BMDL to SNCDL (again for all combination of the BMR and SNR) are also estimated. The extra risk at the SNCDL, SNCD and SNCDU are estimated, as this is not controlled in the estimation of the SNCD. The percentages of extra risks that are smaller than $10 \%, 5 \%$ and $1 \%$ are also reported.

\subsection{Data}

The U.S National Toxicology Program (NTP) 2-year rodent toxicology and carcinogenesis studies are used as the basis for the evaluation of the different PoDs here. The rodent carcinogenicity bioassay program begun by the National Cancer Institute (NCI) has been directed by NTP since 1981. Together, they have conducted over 500 cancer studies. A common protocol in most of test studies is, testing male and female rats and mice at control and three dose levels for each test agent.

Data that is used in this research was abstracted on 2009-01-13 from the Toxicology Database Management System (TDMS) and Carcinogenesis Bioassay Data System (CBDS) by Sand et. al. (2011). We received 1,381 datasets from these authors. We exclude datasets with stop doses and that describing combinations of morphology codes (e.g. "adenoma and carcinoma") if the combined response is only ascribed to the observed response associated with a specific morphology code (e.g. "adenoma" response, but zero "carcinoma" response) already accounted for in the data selection (Sand et. al. (2011) [18]). Consequently, we use 1,128 datasets in our studies.

\subsubsection{Model Fitting}

We fit the Hill model given in Equation 2.1 to these datasets. Benchmark dose is estimated using analytical approach developed in chapter 2 and the signal-to-noise crossover dose and its lower bound using analytical approach with multiple regression bias correction presented in chapter 3. Multiple regression equation to correct the 
bias is obtained using the data simulated in the dose range of zero to eight. As a result, we transform dose range of all datasets (from both the mice and rats data) to be in the range of $(0,8)$ by dividing by an appropriate factor.

Hill model is fitted using mle2 function from bbmle package in $\mathrm{R}$ presented in chapter 2 on these transformed datasets. Datasets demonstrating significant doseresponse trend (i.e. fitted model significantly different from the horizontal line with p-value $\leq 0.05$ ) and adequate goodness-of-fit (i.e. fitted model is not significantly different from the observed data with $\mathrm{p}$-value $\geq 0.05)$ are included in the analysis, leaving a total of 876 (386 mice 490 rats) from 1,128 datasets. Significant doseresponse trend is assessed using likelihood ratio test on fitted Hill model and the horizontal line. Adequate goodness-of-fit test is carried out using likelihood ratio test on the data set and the fitted Hill model. For datasets with background incidence $\leq 0.001$, the background incidence is fixed at the starting values. For some datasets, initial values for model fitting is chosen by observing the scatter plot of data; and for others, fitted Hill model parameter values provided by Sand. et. al. (2011) is used. Maximum likelihood estimates of the Hill model parameters and their asymptotic variance-covariance are obtained for all 876 datasets.

\subsection{Equations and Methods}

In this section, we discuss the equations and methods used to estimate the benchmark dose, lower bound on the benchmark dose, signal-to-noise crossover dose and lower bound on signal-to-noise crossover dose on these datasets.

\subsubsection{Estimates of BMD, BMDL and Ratios}

We estimate the benchmark dose based on extra risk definition (Equation 2.4) for these 876 datasets using Equation 2.20, which is a function of the Hill model parameter estimates $\widehat{\kappa}$ and $\widehat{\eta}$ and benchmark response. We further calculate lower 
bound on the benchmark dose (BMDL) for all these datasets.

The BMDL is estimated using profile likelihood method. Using this method, the upper (one-sided) 95\% confidence bound on the extra risk at a given dose, d, will, if used as the BMR in a standard BMD analysis, result in a BMDL which equals that dose, d (i.e. if the BMDL is defined as the lower one-sided 95\% confidence bound on the BMD, and is estimated under the profile likelihood method) [18]. BMDL is estimated when the $\mathrm{p}$ value for log-likelihood ratio test is less than 0.1 . Whenever this fails, BMDL is not estimated.

Both BMD and BMDL are estimated for BMR of $10 \%, 5 \%$ and $1 \%$. If both BMD and BMDL are available for a dataset, then the corresponding ratio of BMDL to BMD is estimated. The ratio of BMDL to BMD is calculated whenever both BMD and BMDL are available for a dataset. The Table 4.1 shows the numbers of estimates BMD, BMDL and that of their ratios (BMDL/BMD) for BMR of $01 \%, 05 \%$ and $10 \%$.

\begin{tabular}{|l|l|l|l|l|l|l|}
\hline Est & $B M D L_{10}$ & $B M D_{10}$ & $B M D L_{05}$ & $B M D_{05}$ & $B M D L_{01}$ & $B M D_{01}$ \\
\hline count(ratio) & 609 & $876(475)$ & 634 & $876(541)$ & 540 & $876(538)$ \\
\hline
\end{tabular}

Table 4.1: Counts of BMD, BMDL and the Corresponding Ratios

\subsubsection{Estimates of SNCD, SNCDL and Ratios}

We estimate the response at specific dose points as a first step in estimating SNCD and SNCDL using Equation 3.2. Using the analytical approach introduced in chapter 3.3.1, we estimate SNCD and SNCDL for these datasets. Ratio of SNCDL to SNCD is estimated when both SNCDL and SNCD are calculated for a dataset. SNCD is estimated when the dose corresponding to the value of SNR is within the experimental dose range. The Table 4.2 includes the numbers of estimates SNCD, SNDL and that of their ratios (SNDL/SNCD) for $\mathrm{p}=1$ and $2 / 3$. 


\begin{tabular}{|l|l|l|l|l|}
\hline Est & $S N C D L_{1.0}$ & $S N C D_{1.0}$ & $S N C D L_{0.67}$ & $S N C D_{0.67}$ \\
\hline count(ratio) & 793 & $381(371)$ & 841 & $608(592)$ \\
\hline
\end{tabular}

Table 4.2: Counts of SNCD, SNCDL and the Corresponding Ratios

Our simulation results in chapter 3 confirm bias in the estimates of SNCD and SNCDL when analytical approach is applied. Further comparison of different approaches to estimating the bias prove that bias estimation using multiple regression equation is the best among the five approaches that we investigate. We estimate the signal-to-noise crossover dose bias using multiple regression Equations 4.1 and 4.2 obtained in chapter 3 for SNCD and SNCDL estimates of 0.67 and 1.0 respectively.

$$
\begin{aligned}
\text { Bias }_{0.67}= & -1.093+0.21 \kappa+0.46 \eta+0.026(k)+0.00032(n)+0.099\left(d_{k}\right)-0.060 \kappa \eta \\
& -0.003 \kappa(k)-0.00011 \kappa(n)-0.016 \kappa\left(d_{k}\right)-0.0065 \eta(k)-0.00025 \eta(n) \\
& -0.049 \eta\left(d_{k}\right)+0.00005 \kappa \eta(n)+0.0067 \kappa \eta\left(d_{k}\right) \\
\text { Bias }_{1.0}= & -1.084+0.22 \kappa+0.45 \eta+0.02(k)+0.0002(n)+0.11\left(d_{k}\right)-0.068 \kappa \eta \\
& -0.0043 \kappa(k)-0.000054 \kappa(n)-0.002 \kappa(n)-0.016 \kappa\left(d_{k}\right)-0.0002 \eta(n) \\
& -0.053 \eta\left(d_{k}\right)-0.00002(n)\left(d_{k}\right)+0.00002 \kappa \eta(n)+0.0073 \kappa \eta\left(d_{k}\right)
\end{aligned}
$$

where $\kappa$ - hill location parameter, $\eta$ - hill shape parameter, $\mathrm{k}$ - number of doses, $\mathrm{n}$ average number of trials per dose, $d_{k}$ - maximum dose.

Using this estimated bias, the estimates of SNCDL, SNCD and SNCDU are bias corrected. And the ratio of bias corrected SNCDL and SNCD (bcSNCDL/bcSNCD for $\mathrm{p}=1.0,0.67)$ are calculated whenever both estimates are available for a dataset. The bias-corrected SNCD and SNCDL are included in the analysis only when these bias-corrected measures report positive values. The number of bias corrected estimates, and their ratios are tabulated in Tables 4.3 . 


\begin{tabular}{|l|l|l|l|l|}
\hline Est & $b c S N C D L_{1.0}$ & $b c S N C D_{1.0}$ & $b c S N C D L_{0.67}$ & $b c S N C D_{0.67}$ \\
\hline count(ratio) & 630 & $373(339)$ & 519 & $526(395)$ \\
\hline
\end{tabular}

Table 4.3: Counts of Bias-corrected SNCD, SNCDL and the Corresponding Ratios

\subsubsection{Ratios of Different PoDs}

Comparison of signal-to-noise crossover dose to benchmark dose is carried out using results obtained from simulation studies as well as that obtained from fitting Hill model on real datasets. In simulation studies, comparison of point estimates of BMD and SNCD performed. In the other, we investigate the relationship between both the BMD to SNCD and BMDL to SNCDL by comparing these corresponding estimates.

Comparison of estimates of BMD to SNCD are performed for all possible combination (Chapter 2 simulations) of four different Hill model parameter combination that give four distinct shapes of the Hill model curves, two different numbers of dose levels (four and six) and four different numbers of trials per dose $\left(n_{i}=50,100,200\right.$ and 500). Histograms for the ratio of BMD to SNCD are included and percentages of ratios that are greater one are reported as well. Based on these histograms, we investigate how the estimates of these two PoDs change with the shape of histograms and the shape of the Hill curves, number of dose levels and number of trials per dose here.

On our real datasets, estimates of BMD and BMDL of BMR of 01\%, 05\%, 10\%; that of SNCDL and SNCD of $\mathrm{p}=1,2 / 3$; and the corresponding bias corrected SNCDL and SNCD are obtained for each dataset if possible. To compare the estimate of BMD with that of SNCD and BMDL with that of SNCDL, ratio of BMD and SNCD and that of BMDL and SNCDL are calculated for each dataset when these corresponding estimates are available for that dataset.

Six different BMD/SNCD (all combination of $\mathrm{p}=1,0.67$ and BMR of 1\%, 
$5 \%, 10 \%$ ), BMD/bcSNCD (all combination of $\mathrm{p}=1.0,0.67$ and BMR of 1\%, 5\%, $10 \%$ ), BMDL/SNCDL (all combination of $\mathrm{p}=1.0,0.67$ and BMR of 1\%, 5\%, 10\%) and BMDL/bcSNCDL (all combination of $\mathrm{p}=1.0,0.67$ and BMR of 1\%, 5\%, 10\%) are estimated for all possible datasets. The percentage of dataset that has this ratio greater than one is also calculated for all different ratios. The corresponding histograms combined with box plots are reported for these ratios of different PoDs as well.

\subsubsection{Extra Risk at SNCDL, SNCD and SNCDU}

In dose-response analysis, quantifying the risk at a PoD is important. Risk is controlled when estimating the benchmark dose, but not in the case of estimation of the SNCD or SNCDL. Consequently, we derive an equation to estimate the extra risk (i.e. SNCR -signal-to-noise crossover response) as a function of estimate of Hill model parameters and of the PoD (either SNCD or SNCDL).

$$
\operatorname{ExtraRisk}_{\theta}=\left(\frac{\theta^{\widehat{\eta}}}{\widehat{\kappa}^{\widehat{\eta}}+\theta^{\widehat{\eta}}}\right)
$$

where $\widehat{\alpha}$ - MLE of background incident, $\widehat{\kappa}$ - MLEs of location parameter, $\widehat{\eta}$ - MLEs of shape parameter, $\theta$ - uncorrected or bias corrected SNCDL, SNCD or SNCDU.

Extra risk is estimated at uncorrected and bias corrected SNCDL, SNCD, SNCDU (for $\mathrm{p}=1.0,0.67$ ) for all datasets. Histograms of extra risk at SNCDL, SNCD, and SNCDU are obtained. Percentage of datasets with extra risk less than 0.1, 0.05 and 0.01 are estimated for all possible cases of estimates of SNCDL, SNCD and SNCDU as we are comparing SNCD with BMD and in the estimation of BMD, extra risk at the BMD is fixed at the traditional values of $0.1,0.05$ and 0.01 . 


\subsection{Results and Discussion}

Here, we present the results of comparing signal-to-noise crossover dose to benchmark dose calculated in our simulation studies and in our studies carried out by fitting the Hill model to the NTP datasets. Results for comparing BMDL to BMD, SNCDL to SNCD and BMD to SNCD of both the point and lower bound estimates are presented.

\subsubsection{Bench Mark Dose Ratios}

\begin{tabular}{|l|l|l|l|l|l|l|}
\hline BMDL/BMD & P05 & P25 & Median & P75 & P95 & IQR \\
\hline $10 \%(475)$ & 0.045 & 0.239 & 0.516 & 0.670 & 0.789 & 0.431 \\
\hline $05 \%(541)$ & 0.027 & 0.192 & 0.413 & 0.586 & 0.758 & 0.394 \\
\hline $01 \%(538)$ & 0.013 & 0.097 & 0.216 & 0.339 & 0.485 & 0.242 \\
\hline
\end{tabular}

Table 4.4: Quantiles of Ratios of BMDL to BMD

Table 4.4 presents the results of comparing lower bound of BMD to BMD where P05 ( $5^{\text {th }}$ percentile), lower quartile, median, upper quartile and P95 (95 ${ }^{\text {th }}$ percentile) for the ratio of BMDL to BMD are included. Ratio of BMDL to BMD is calculated for 475 datasets for BMR of 10\%, 541 for that of $5 \%$ and 538 for that of $1 \%$. Histogram for this ratio for BMR values of $10 \%, 5 \%$ and $1 \%$ are presented Figure 4.1 .

Median for BMR of $10 \%$ is 0.516 , that of $05 \%$ is 0.413 and that of $01 \%$ is 0.216 and. These results show that median decreases with decreasing BMR. And figure 4.1 shows that the skewness of the histogram is moving from left to right as the BMR decreases. As a result, we can say that confidence interval for BMD becomes wider as the value of BMR goes down. i.e. BMDL is much smaller than the corresponding BMD for smaller BMRs and variability of BMD increases with decreasing BMR.

Inter quartile range (IQR) for BMR of $10 \%$ is 0.431 , that of $05 \%$ is 0.394 and that of $01 \%$ is 0.242 . We observe that IQR decreases with decreasing BMR. This 




(a) BMDL10/BMD10

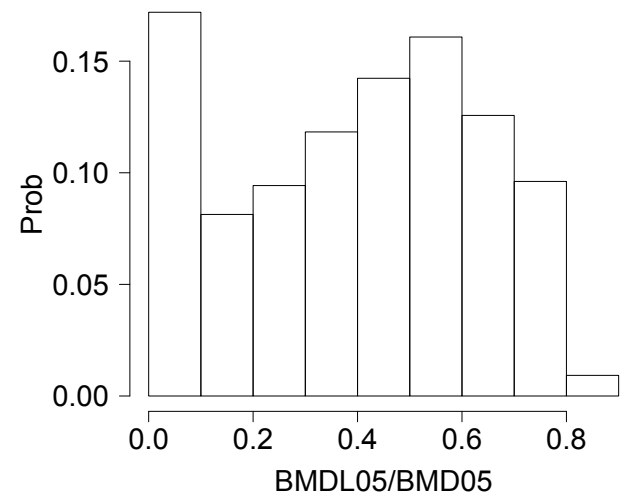

(b) BMDL05/BMD05

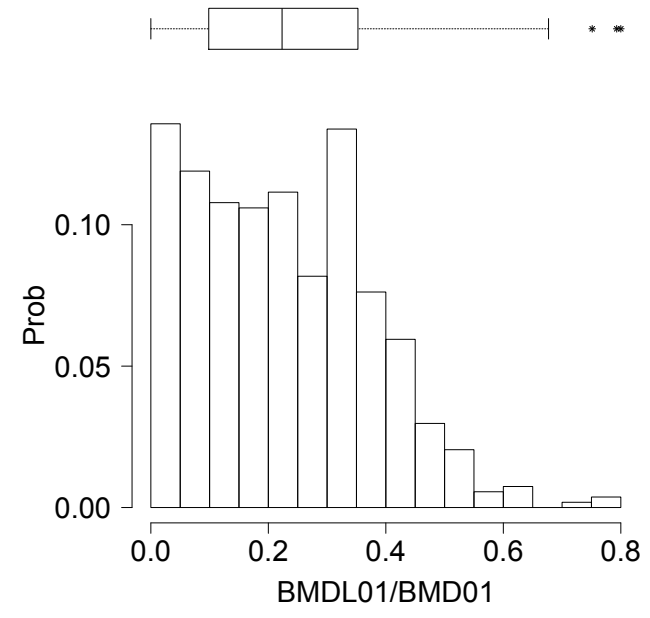

(c) BMDL01/BMD01

Figure 4.1: Histogram for Ratios of BMDL to BMD

implies that variability for this ratio is less or confidence interval is tighter for this ratio with smaller BMR values. 


\subsubsection{Signal-to-noise Crossover Dose Ratios}

We here present results of comparing SNCDL to SNCD of both the uncorrected and bias-corrected estimates.

\subsubsection{Uncorrected}

\begin{tabular}{|l|l|l|l|l|l|l|}
\hline SNCDL/SNCD & P05 & P25 & Median & P75 & P95 & IQR \\
\hline $1.0(371)$ & 0.009 & 0.431 & 0.641 & 0.764 & 0.873 & 0.333 \\
\hline $0.67(592)$ & 0.008 & 0.389 & 0.593 & 0.736 & 0.868 & 0.347 \\
\hline
\end{tabular}

Table 4.5: Quantiles for Ratios of SNCDL to SNCD

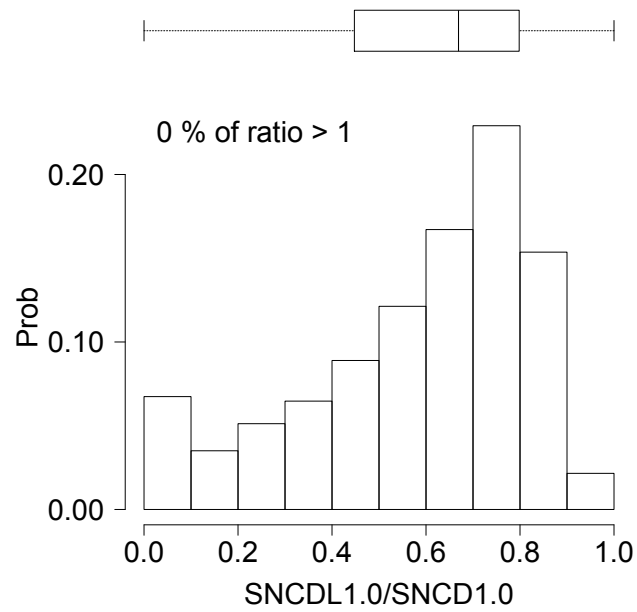

(a) SNCDL1.0/SNCD1.0



(b) SNCDL0.67/SNCD0.67

Figure 4.2: Histogram for Ratios (uncorrected) of SNCDL to SNCD

Table 4.5 presents the P05, lower quartile, median, upper quartile and P95 for ratios of SNCDL to SNCD. The ratio of SNCDL to SNCD of $p=1.0$ is estimated for 371 datasets and that of $\mathrm{p}=0.67$ is for 592 datasets. Figure 4.2 includes the histogram and box plot for the ratio of SNCDL and SNCD estimates calculated on NTP datasets. 
Median of $\mathrm{p}$ equals to 1 and 0.67 are 0.641 and 0.593 . Median is smaller for the ratio estimated with smaller SNR. This confirms that variability is higher or confidence interval is wider for SNCD with smaller SNR. However, medians for these ratios of signal-to-noise crossover dose is larger than what is observed for the corresponding ratio of benchmark dose. Therefore, we can say that the confidence interval of SNCD (i.e. length of SNCDL to SNCDU) is narrower than that for BMD.

Inter quartile range (IQR) of p equals to 1 and 0.67 are 0.333 and 0.347 . As IQR of both these ratios are tighter than that for this ratio of BMDL and BMD of BMR of $10 \%$ and $05 \%$, we see that variability for this ratio is smaller than that for benchmark dose. Panels (a) and (b) of figure 4.2 shows that shape of the histogram moves slowly from left to right as the value of $\mathrm{p}$ goes down just as the shape of the histogram for the ratio BMDL to BMD moves from left to right as BMR decreases. i.e. length of the confidence interval for SNCD increases as p goes down but slowly as opposed corresponding characteristics for benchmark dose.

\subsubsection{Bias-corrected}

\begin{tabular}{|l|l|l|l|l|l|l|}
\hline bcSNCDL/bcSNCD & P05 & P25 & Median & P75 & P95 & IQR \\
\hline $1.0(339)$ & 0.065 & 0.408 & 0.618 & 0.745 & 0.873 & 0.337 \\
\hline $0.67(395)$ & 0.088 & 0.379 & 0.578 & 0.718 & 0.871 & 0.339 \\
\hline
\end{tabular}

Table 4.6: Quantiles for Ratios of Bias-corrected SNCDL to SNCD

Table 4.6 includes similar results as above for bias corrected estimates of SNCDL, SNCD. Figure 4.3 includes the histogram for the ratio of bias-corrected SNCDL to SNCD. Median for the ratio with SNR of 1 is 0.618 and that with SNR of 0.67 is 0.578 . Shapes of the histograms are more symmetric than that from uncorrected. IQR is the same as the one obtained for uncorrected ones. We observe more symmetry in the histogram of SNCDL to SNCD of $\mathrm{p}=2 / 3$ when bias corrected. Overall, we observe similar characteristics as the uncorrected but with smaller medians. 


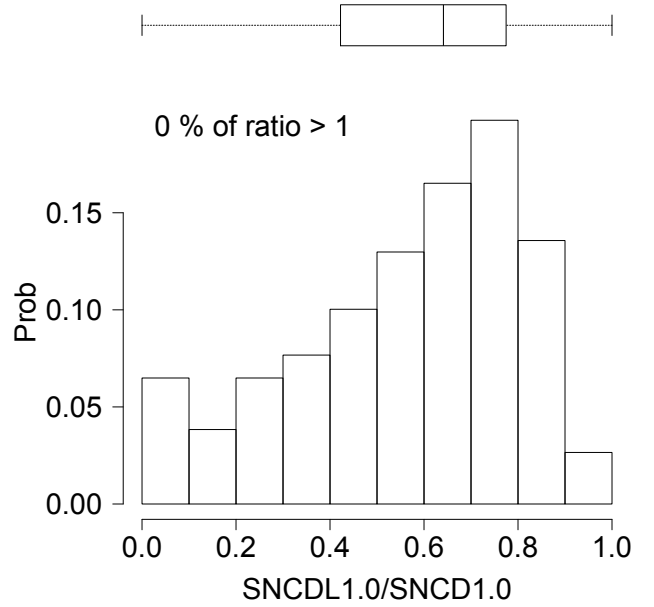

(a) bcSNCDL1.0/bcSNCD1.0

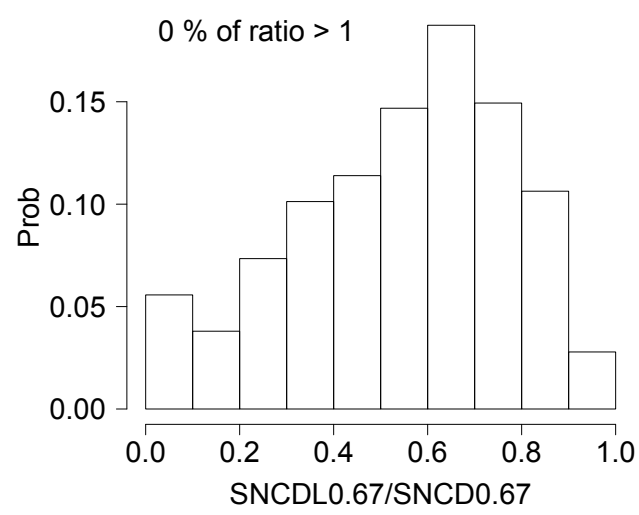

(b) bcSNCDL0.67/bcSNCD0.67

Figure 4.3: Histogram for Ratios (bias-corrected) of SNCDL to SNCD

\subsubsection{Ratios of BMD to SNCD}

Ratios of BMD to SNCD are presented for simulated data as well as for real datasets. For real datasets, the results for ratios of BMD to SNCD are presented for both uncorrected and bias corrected SNCD.

\subsubsection{Simulation Results of Ratio of BMD to SNCD}

Simulation studies are carried out for ratios of BMD to SNCD of all possible combination of BMR $(10 \%, 5 \%$ and 1\%) and values of $\mathrm{p}(1$ and $2 / 3)$ based on all possible combinations of four selected combination of Hill Model parameter values, two different number of dose levels (four and six), and four different numbers of trials per dose $(50,100,200$ and 500). We present the corresponding histograms and the percentages that are greater than one for all thirty-two different scenarios.

Figures 4.4, 4.5, 4.6 and 4.7 show the histograms for the ratio of BMD to SNCD for four different Hill model parameter combinations with four doses and 50 trials per dose. The histograms are almost symmetric for S-shaped and concave with initial slope greater than zero curves. Shape of the histogram further becomes symmetric 

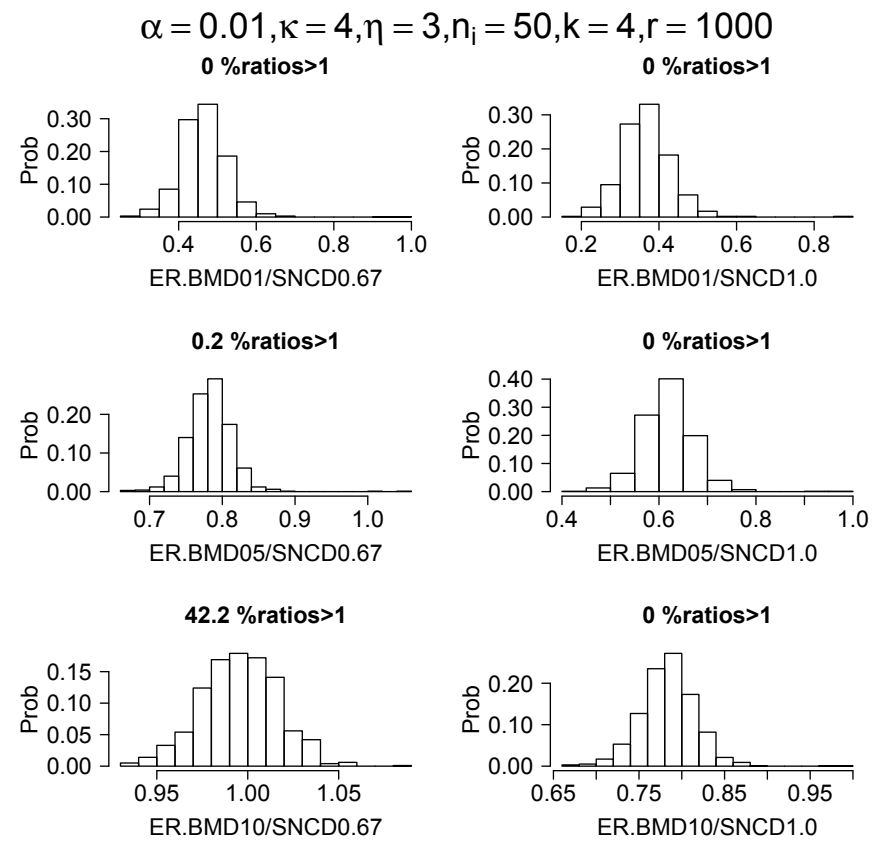

Figure 4.4: Histogram for Ratios of BMD to SNCD estimated on S-shaped Hill Model Curves
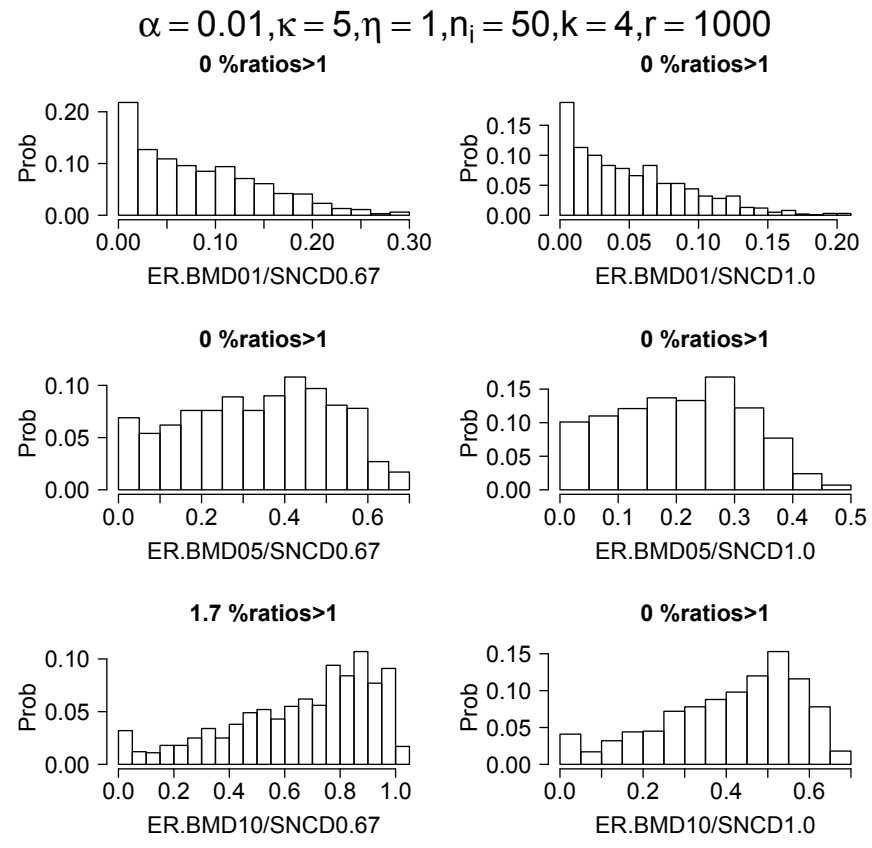

Figure 4.5: Histogram for Ratios of BMD to SNCD estimated on Convex Hill Model Curves 

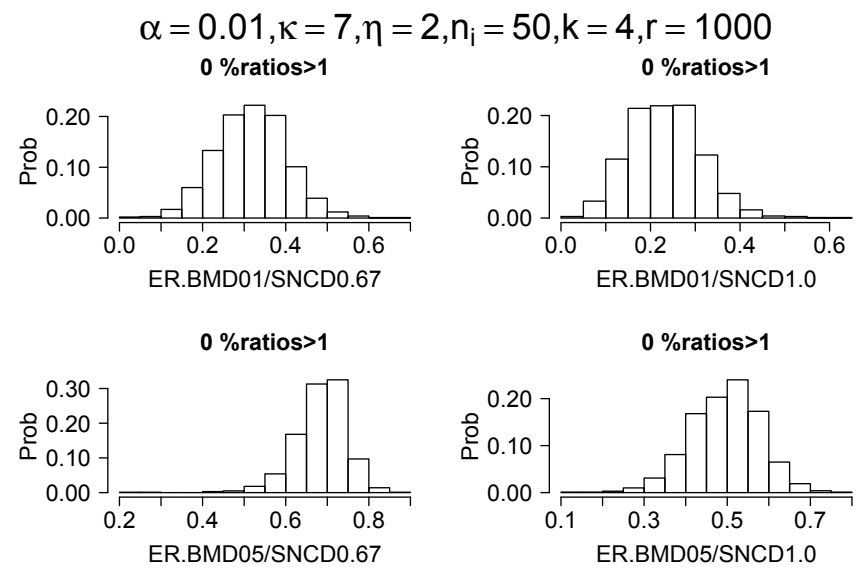

$20 \%$ ratios $>1$


Figure 4.6: Histogram for Ratios of BMD to SNCD estimated on initial slope $>0$ Hill Model Curves
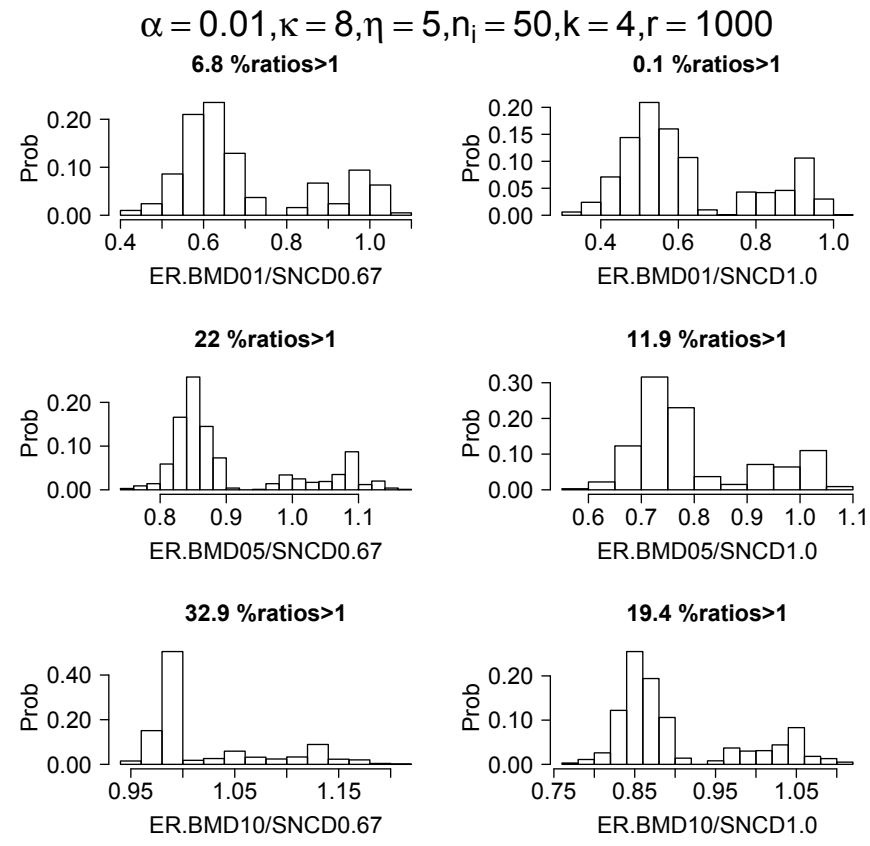

Figure 4.7: Histogram for Ratios of BMD to SNCD estimated on initial slope=0 Hill Model Curves 
for these two when either number of dose levels or that of trials per dose is increased. Histograms are right-skewed for convex curve with four doses and 50 trials per dose. This histogram becomes symmetric with increasing number of trials than that of number of dose levels for convex curve (Figures C.2 and C.10). On the other hand, shape of the histogram is bimodal for concave with initial slope zero with four doses and 50 trials per dose. Histogram for this curve become symmetric with increasing number of dose levels than that of number of trials per dose (Figures C.12 and C.4). We observe similar characteristics based on shape of the Hill curves, number of dose levels and number of trials per dose for any estimates in chapter 2 and 3.

Further, higher the BMR of BMD and/or p of SNCD, the more symmetric the histogram for corresponding shape of the Hill curves. Percentages greater than one increases with increasing number of dose levels and number of trials per dose for

the corresponding Hill curves (Figures in Appendix C). This observation confirms that signal-to-noise crossover dose decreases with increasing number of dose levels or number of trials per dose.

\subsubsection{Results for Ratio of BMD to SNCD from Real Datasets}

We estimate BMD for BMR of 10\%, 05\% and 01\% and SNCD for p of 1.0 and 0.67 on the NTP datasets. As a result, six different ratios of BMD to SNCD are obtained for each of uncorrected and bias corrected SNCD estimates on these 876 datasets that we analyze.

\section{Ratio of BMD to Uncorrected SNCD}

Table 4.7 presents the quantiles and counts for different ratios of BMD to uncorrected SNCD for all possible combination of BMR of $10 \%, 5 \%$ and $1 \%$ and SNR of 1 and 0.67. The number of datasets estimated for these six different ratios are given in the first column. Figures 4.8, 4.9 and 4.10 includes the histogram and boxplot obtained 


\begin{tabular}{|l|l|l|l|l|l|l|}
\hline BMD/SNCD & P05 & P25 & Median & P75 & P95 & IQR \\
\hline $10 / 1.0(361)$ & 0.081 & 0.379 & 0.582 & 0.753 & 1.068 & 0.374 \\
\hline $05 / 1.0(370)$ & 0.030 & 0.187 & 0.376 & 0.560 & 1.005 & 0.373 \\
\hline $01 / 1.0(359)$ & 0.002 & 0.038 & 0.129 & 0.253 & 0.547 & 0.215 \\
\hline $10 / 0.67(487)$ & 0.287 & 0.696 & 0.977 & 1.075 & 1.374 & 0.379 \\
\hline $05 / 0.67(579)$ & 0.071 & 0.369 & 0.597 & 0.778 & 1.041 & 0.409 \\
\hline $01 / 0.67(571)$ & 0.003 & 0.075 & 0.189 & 0.385 & 0.859 & 0.310 \\
\hline
\end{tabular}

Table 4.7: Quantiles for Ratios of BMD to SNCD

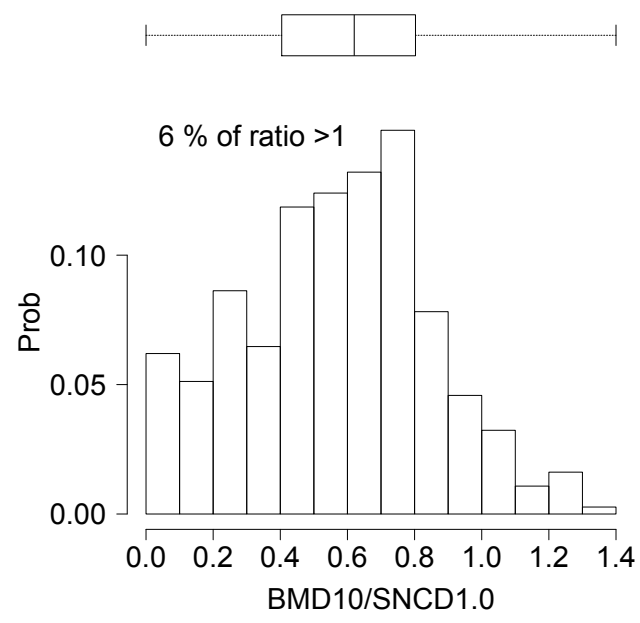

(a) BMD10/SNCD1.0

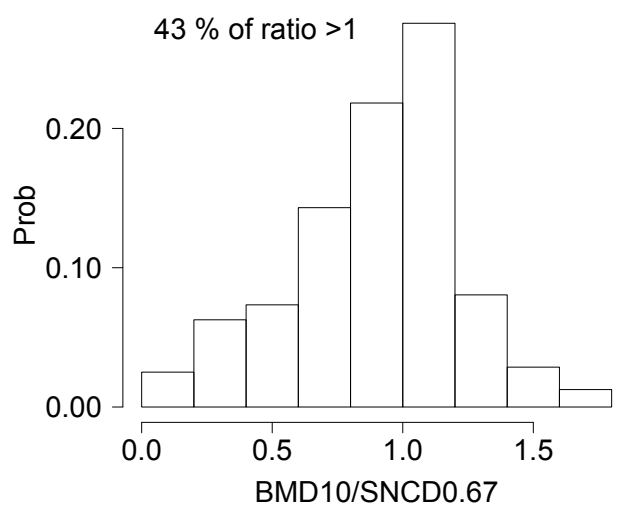

(b) BMD10/SNCD0.67

Figure 4.8: Histogram for Ratios of BMD10 to SNCD

for these ratios.

The results in table 4.7 for medians for all possible combinations of ratio of BMD to SNCD show that median for this ratio decreases with decreasing BMR for a SNCD with specific p, but increases with decreasing SNR for a specific BMR. Histogram in figures $4.8,4.9$ and 4.10 show that shapes of these histograms change from symmetric to right-skewed as the value of BMR decreases. But, the shape is more symmetric (or less right-skewed) for ratio of any BMD to SNCD0.67 than that to SNCD1.0.

When we investigate the median for ratio of largest of BMD to largest SNCD, i.e. BMD10 to SNCD1.0, we observe this median is 0.582 . This implies that $50 \%$ 


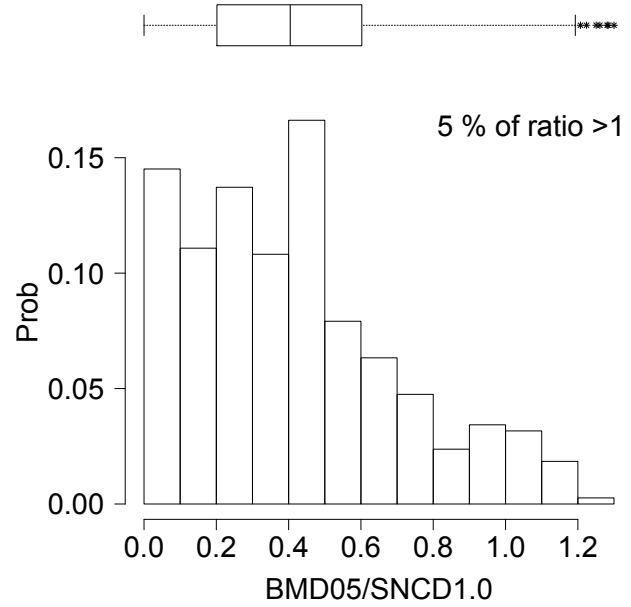

(a) BMD05/SNCD1.0

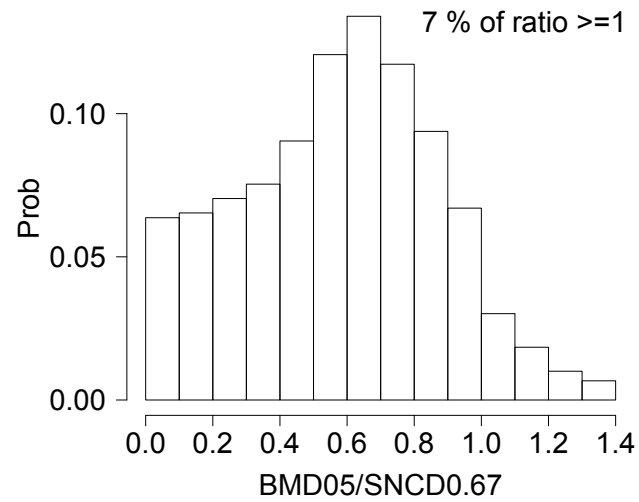

(b) BMD05/SNCD0.67

Figure 4.9: Histogram for Ratios of BMD05 to SNCD

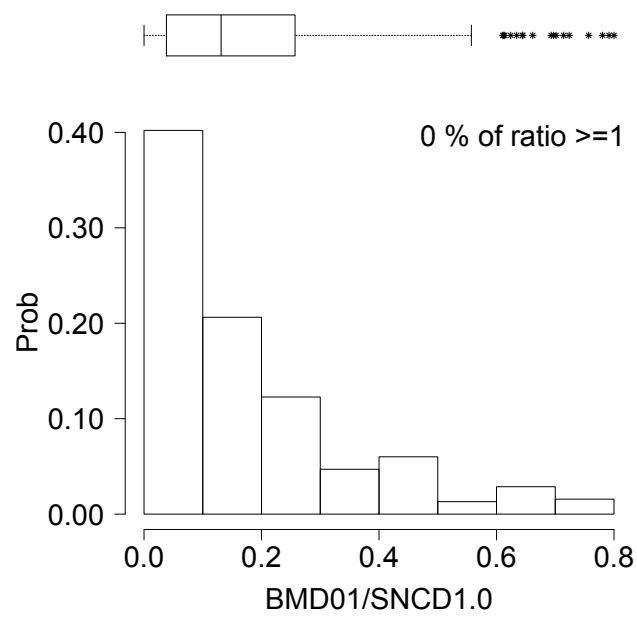

(a) BMD01/SNCD1.0



(b) BMD01/SNCD0.67

Figure 4.10: Histogram for Ratios of BMD01 to SNCD

of BMD10 are smaller than 0.582 times the SNCD1.0. Consequently, SNCD1.0 is relatively larger than BMD10 for most of the datasets. Hence to find a comparable BMD to SNCD, we investigate ratio of BMD10 to the next smallest SNCD which is SNCD0.67. We observe that median for BMD10 to SNCD0.67 of 0.977 which implies that $50 \%$ of SNCD0.67 are smaller than BMD10. Further investigation of panel (b) 
of figure 4.8 shows that only $43 \%$ of SNCD0.67 greater than BMD10 (i.e. $57 \%$ of SNCD0.67 are smaller than BMD10). As a result, We can say that the estimate of uncorrected SNCD0.67 is comparable to BMD10.

\section{Ratio of BMD to Bias-corrected SNCD}

In chapter 3, our simulation results confirm that bias-corrected SNCD estimates perform better than uncorrected. Hence, we carry out similar comparison BMD to SNCD but with bias-corrected SNCD. Table 4.8 presents similar results as above but with bias corrected SNCD. Figures 4.11, 4.12 and 4.13 presents histogram for the ratio of BMD to bias-corrected SNCD.

\begin{tabular}{|l|l|l|l|l|l|l|}
\hline BMD/bcSNCD & P05 & P25 & Median & P75 & P95 & IQR \\
\hline $10 / 1.0(358)$ & 0.100 & 0.420 & 0.666 & 0.812 & 1.050 & 0.392 \\
\hline $05 / 1.0(372)$ & 0.035 & 0.232 & 0.415 & 0.579 & 0.899 & 0.347 \\
\hline $01 / 1.0(353)$ & 0.004 & 0.046 & 0.133 & 0.229 & 0.476 & 0.183 \\
\hline $10 / 0.67(439)$ & 0.230 & 0.745 & 1.058 & 1.232 & 1.636 & 0.487 \\
\hline $05 / 0.67(472)$ & 0.115 & 0.535 & 0.815 & 0.945 & 1.254 & 0.410 \\
\hline $01 / 0.67(509)$ & 0.022 & 0.170 & 0.290 & 0.482 & 0.889 & 0.312 \\
\hline
\end{tabular}

Table 4.8: Quantiles for Ratios of BMD to Bias-corrected SNCD

Median of the corresponding ratio of BMD to SNCD in table 4.8 is higher than that in table 4.7. We have a median of 0.666 for ratio of BMD10 to SNCD1.0. This implies that $50 \%$ of BMD10 are smaller than 0.666 times (instead of 0.582 with uncorrected) the SNCD1.0. Although there is an improvement due to bias-correction, it is not significant enough to change the previous findings. Although median for the ratio of BMD10 to SNCD0.67 is 1.058 (larger than one), that for ratio of next smallest BMD, i.e. BMD05, to SNCD0.67 is only 0.815 which is a significant improvement from corresponding ratio obtained using uncorrected measure, but again not significant enough to change the findings. These results confirm that bias-corrected SNCD0.67 is comparable only to BMD10 as before. 
We observe a similar change in the shape of the histogram with decreasing BMR or SNR as above however the shape of the corresponding ratio tends more toward symmetry in the case bias-corrected SNCD. In panel (b) of figure 4.12 , having $61 \%$ of this ratio greater than 1 implies that $61 \%$ of bias-corrected SNCD0.67 are smaller than BMD10. The percentage of ratio greater than one improves for BMD10/SNCD0.67 from $43 \%$ to $61 \%$ when bias-corrected. A similar trend is observed for the ratio of BMD05/SNCD0.67 (change is from 7\% to 17\%). However, there is no significant improvement for the ratio of BMD01/SNCD0.67 (or BMD01/SNCD1.0) (i.e. percentage of ratio greater than 1.0 remained the same). These results confirm that SNCD0.67 remains comparable to BMD10.

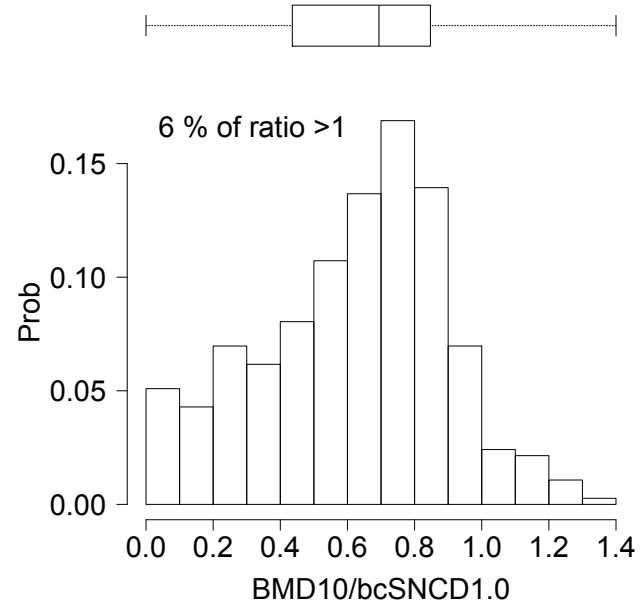

(a) BMD10/bcSNCD1.0

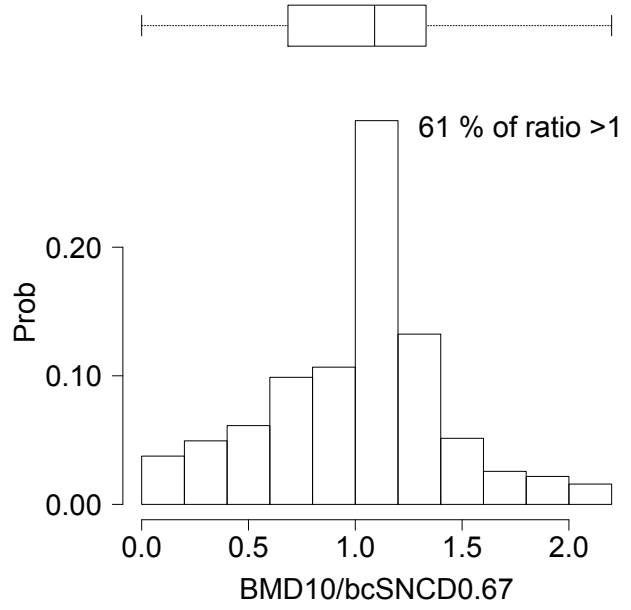

(b) BMD10/bcSNCD0.67

Figure 4.11: Histogram for Ratios of BMD10 to Bias-corrected SNCD

Histogram is right-skewed more as the value of BMR goes down for the corresponding value of $\mathrm{p}$ for SNCD estimates, but moves toward symmetry as the value of $\mathrm{p}$ goes down for same BMR value. Consequently, this characteristics points out the need to estimate SNCD with lower value of $\mathrm{p}($ i.e. $<2 / 3)$ to get a comparable estimate for BMD of $05 \%$ and $01 \%$. 


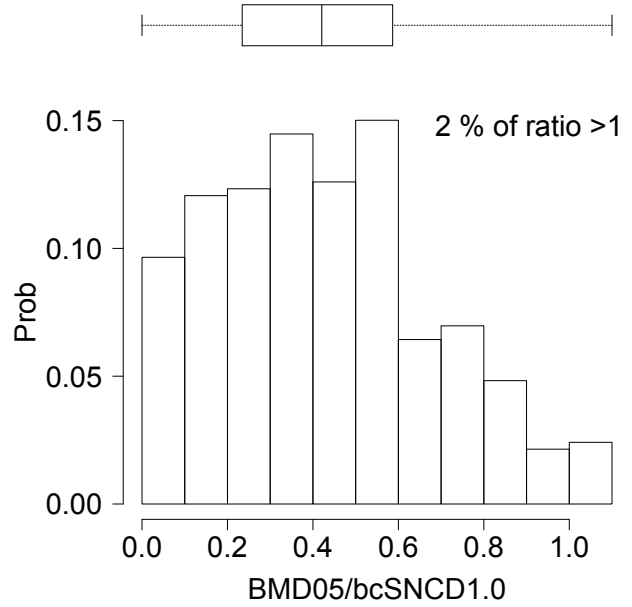

(a) BMD05/bcSNCD1.0

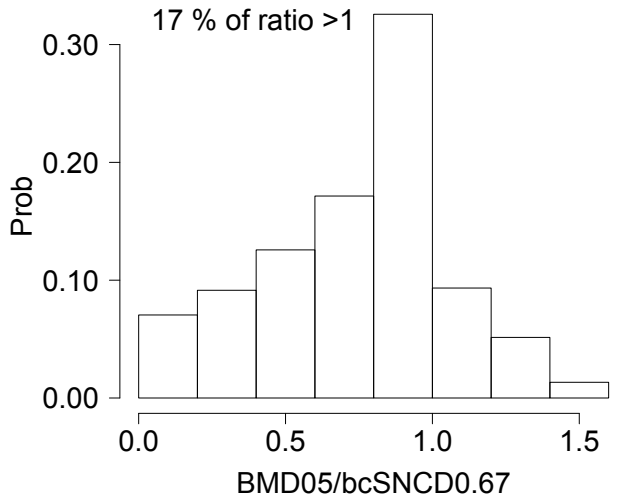

(b) BMD05/bcSNCD0.67

Figure 4.12: Histogram for Ratios of BMD05 to Bias-corrected SNCD

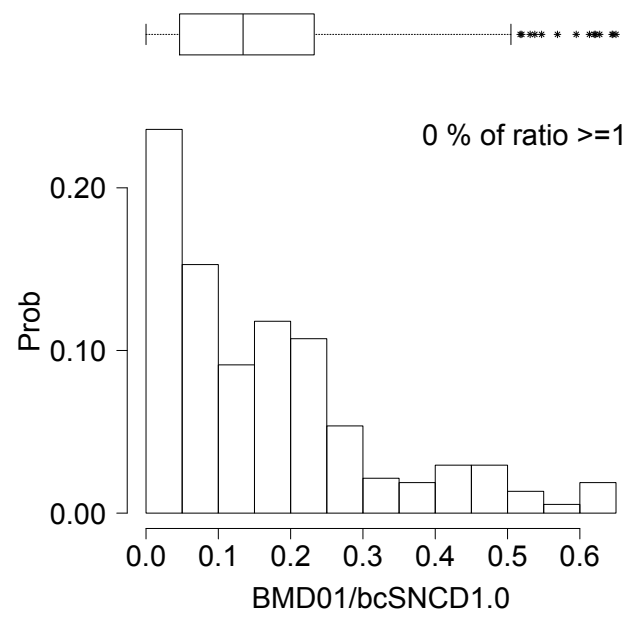

(a) BMD01/bcSNCD1.0

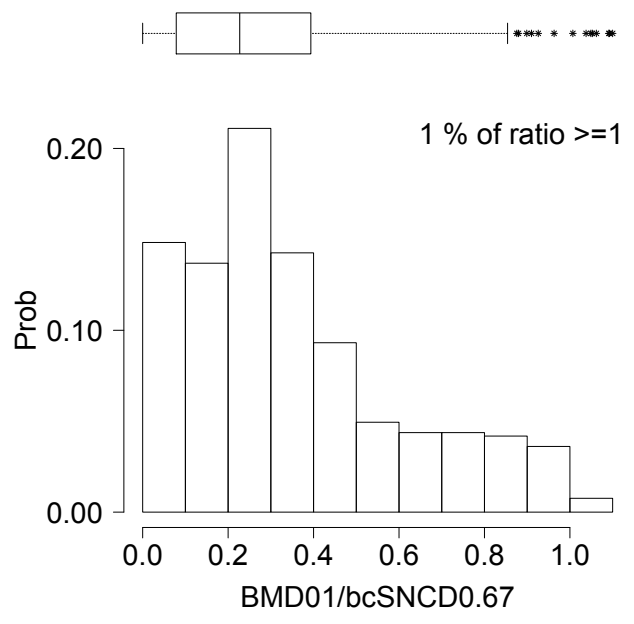

(b) BMD01/bcSNCD0.67

Figure 4.13: Histogram for Ratios of BMD01 to Bias-corrected SNCD

\subsubsection{Ratios of BMDL and SNCDL}

Here, we compare the lower bound on signal-to-noise crossover dose with the that of benchmark dose to identify the SNR value of SNCDL that is comparable to the BMR values of BMDL. Hence, the ratio of BMDL to SNCDL for all possible combinations of BMR of $10 \%, 05 \%$ and $01 \%$ and $\mathrm{p}$ of 1 and $2 / 3$ is calculated for 
estimates of BMDL and SNCDL calculated on these real datasets. The percentage of ratio greater than one is reported as well. We report the same results when biascorrected SNCDL estimates is used.

\subsubsection{Ratio of BMDL to Uncorrected SNCDL}

\begin{tabular}{|l|l|l|l|l|l|l|}
\hline BMDL/SNCDL & P05 & P25 & Median & P75 & P95 & IQR \\
\hline $10 / 1.0(475)$ & 0.042 & 0.271 & 0.573 & 0.914 & 1.546 & 0.643 \\
\hline $05 / 1.0(487)$ & 0.010 & 0.117 & 0.336 & 0.672 & 1.291 & 0.555 \\
\hline $01 / 1.0(199)$ & 0.003 & 0.025 & 0.062 & 0.133 & 0.293 & 0.108 \\
\hline $10 / 0.67(496)$ & 0.064 & 0.452 & 0.897 & 1.317 & 2.410 & 0.865 \\
\hline $05 / 0.67(493)$ & 0.019 & 0.204 & 0.488 & 0.891 & 1.506 & 0.687 \\
\hline $01 / 0.67(423)$ & 0.006 & 0.043 & 0.136 & 0.408 & 1.271 & 0.365 \\
\hline
\end{tabular}

Table 4.9: Quantiles for Ratios of BMDL to SNCDL

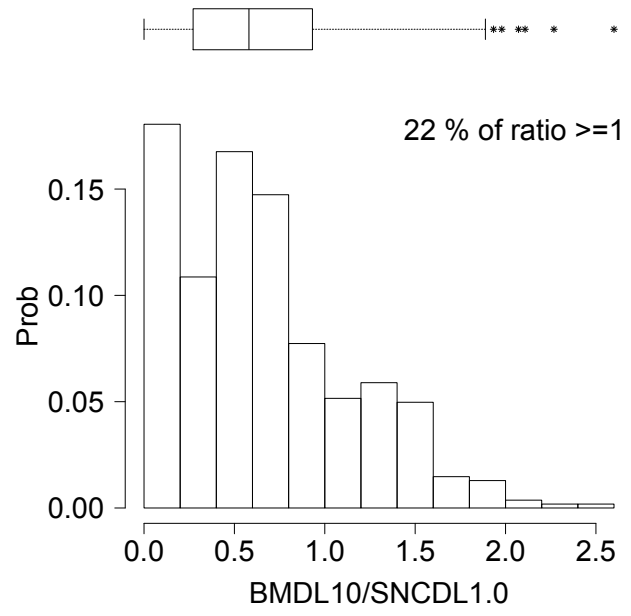

(a) BMDL10/SNCDL1.0

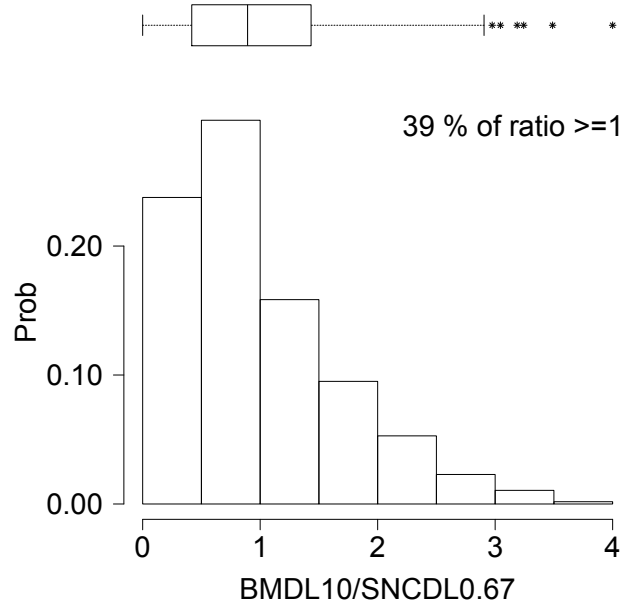

(b) BMDL10/SNCDL0.67

Figure 4.14: Histogram for Ratios of BMDL10 to SNCDL

Table 4.9 presents the quantiles for all possible combinations of the ratio of BMDL to SNCDL. Histograms and box plots for these ratios are presented in figures 4.15, 4.14 and 4.16 . 


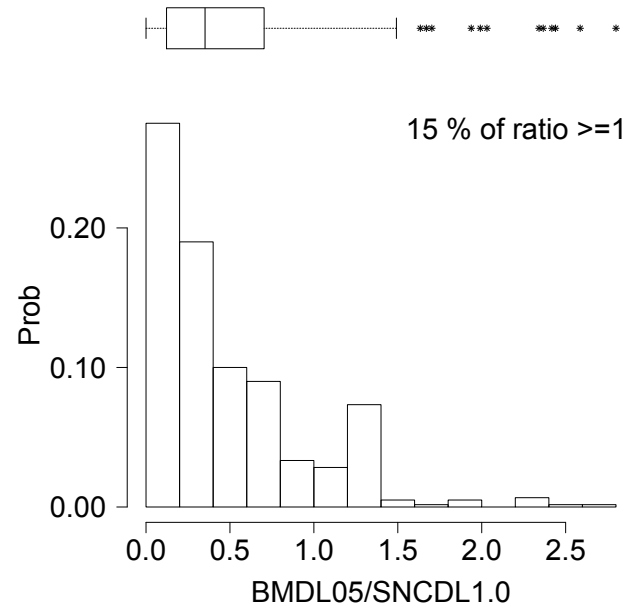

(a) BMDL05/SNCDL1.0

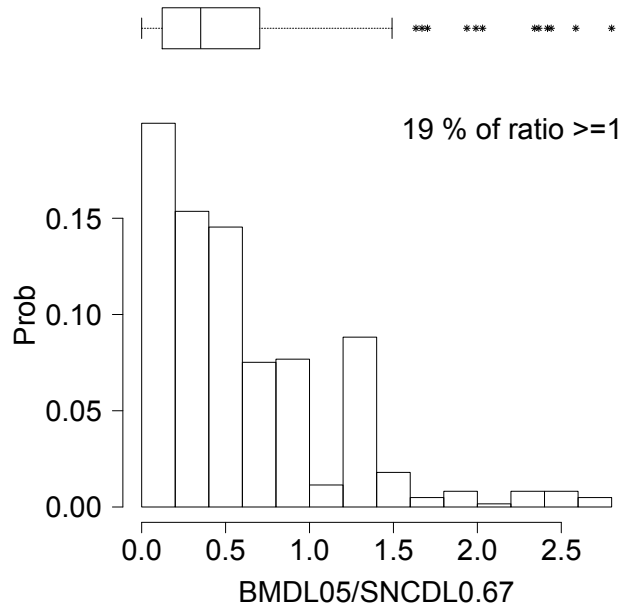

(b) BMDL05/SNCDL0.67

Figure 4.15: Histogram for Ratios of BMDL05 to SNCDL

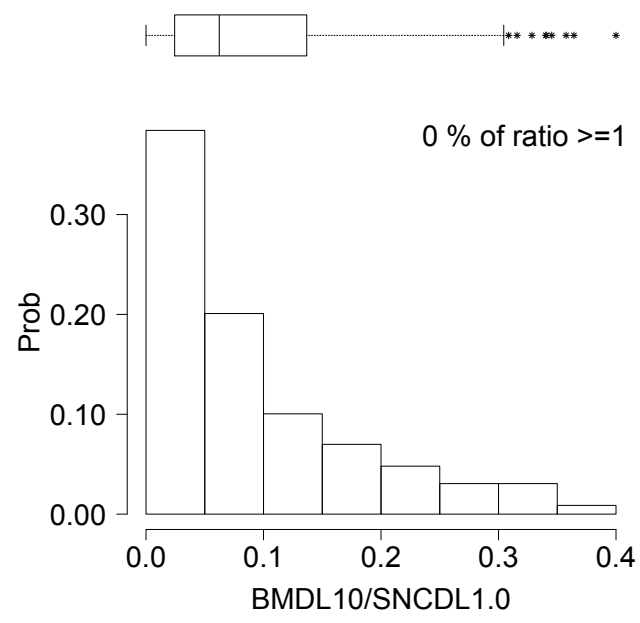

(a) BMDL01/SNCDL1.0

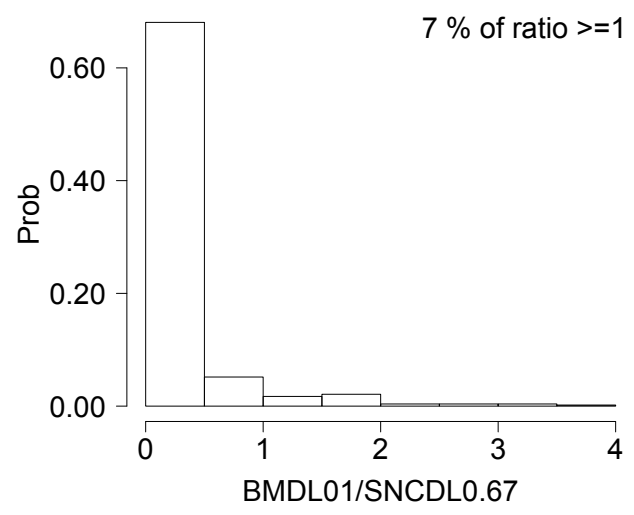

(b) BMDL01/SNCDL0.67

Figure 4.16: Histogram for Ratios of BMDL01 to SNCDL

We see that none of the medians is close to or greater than one among these six different ratios of BMDL to uncorrected SNCDL (Table 4.9). Shape of the histograms of all possible ratios are right-skewed. Skewness of the histogram moves very slowly from right to symmetric when the value of SNR for SNCDL goes down, and moves to more pronounced right-skewed with decreasing BMR. We have seen similar behavior 
with higher degree for the ratio of BMD to SNCD. The largest median observed is 0.897 (smaller than one) for ratio of BMD10 to SNCD0.67. Only 39\% of SNCDL0.67 are smaller than BMDL10 (Figure 4.15). We can say that none of the lower bound of SNCD is comparable to any of that of BMD before bias-correction.

\subsubsection{Ratio of BMDL to Bias-corrected SNCDL}

\begin{tabular}{|l|l|l|l|l|l|l|}
\hline BMDL/bcSNCDL & P05 & P25 & Median & P75 & P95 & IQR \\
\hline $10 / 1.0(411)$ & 0.044 & 0.335 & 0.626 & 0.918 & 1.427 & 0.583 \\
\hline $05 / 1.0(458)$ & 0.016 & 0.165 & 0.378 & 0.716 & 1.324 & 0.551 \\
\hline $01 / 1.0(202)$ & 0.004 & 0.025 & 0.063 & 0.131 & 0.266 & 0.106 \\
\hline $10 / 0.67(341)$ & 0.060 & 0.469 & 0.971 & 1.342 & 2.487 & 0.873 \\
\hline $05 / 0.67(394)$ & 0.032 & 0.326 & 0.615 & 0.989 & 1.515 & 0.663 \\
\hline $01 / 0.67(365)$ & 0.012 & 0.061 & 0.150 & 0.372 & 0.522 & 0.311 \\
\hline
\end{tabular}

Table 4.10: Quantiles for Ratios of BMDL to Bias-corrected SNCDL

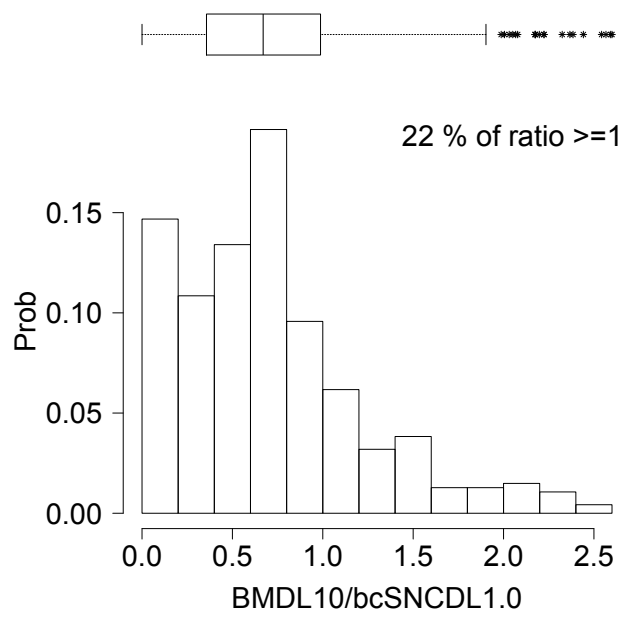

(a) BMDL10/bcSNCDL1.0

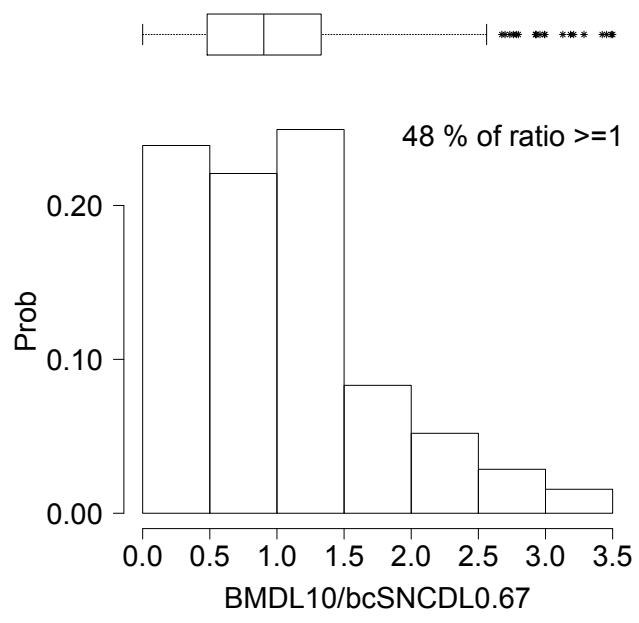

(b) BMDL10/bcSNCDL0.67

Figure 4.17: Histogram for Ratios of BMDL10 to Bias-corrected SNCDL

We present the same results that is presented in section 4.4.4.1 but with biascorrected SNCDLs. Table 4.10 presents P05, lower quantile, median, upper quartile, 


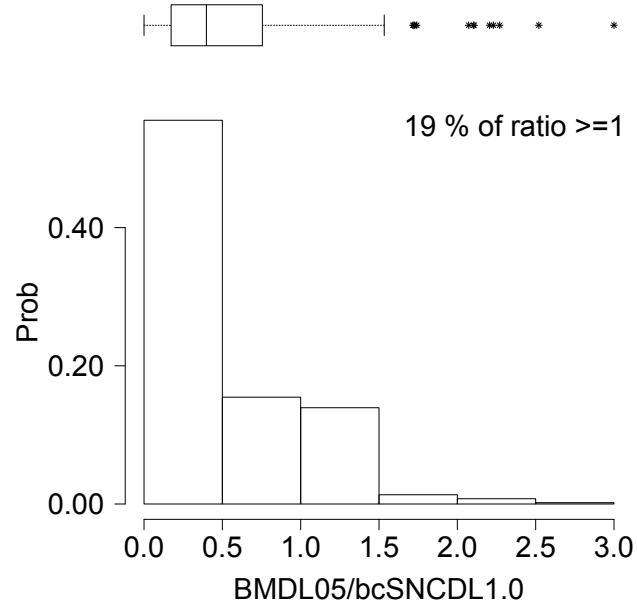

(a) BMDL05/bcSNCDL1.0

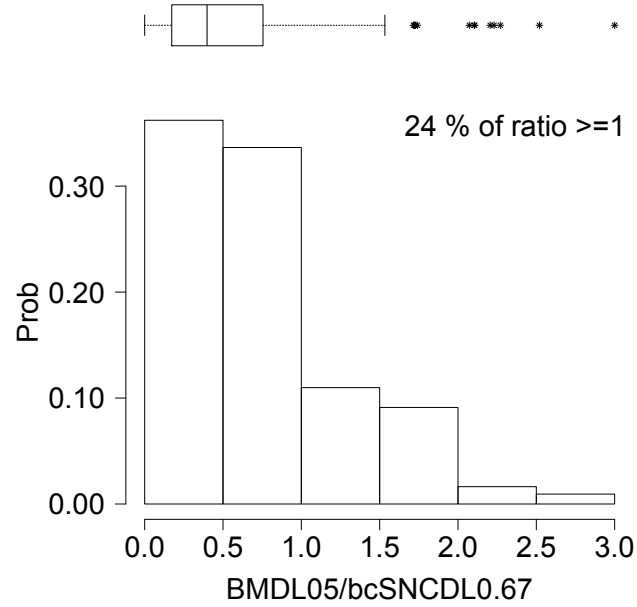

(b) BMDL05/bcSNCDL0.67

Figure 4.18: Histogram for Ratios of BMDL05 to Bias-corrected SNCDL

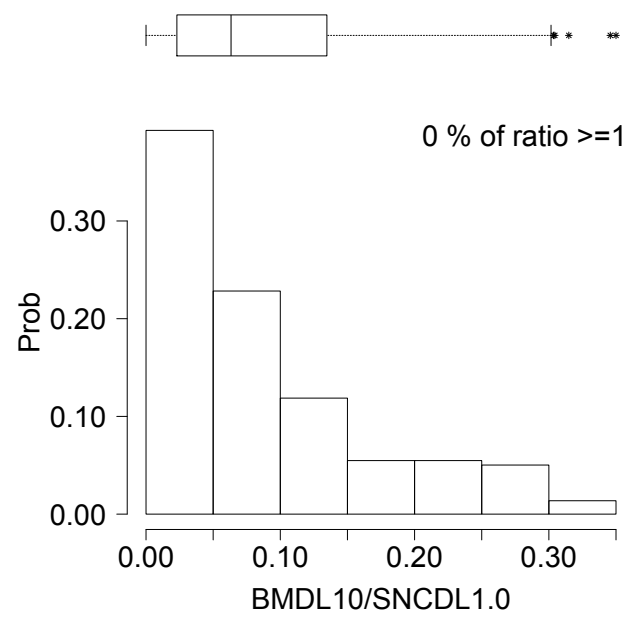

(a) BMDL01/bcSNCDL1.0

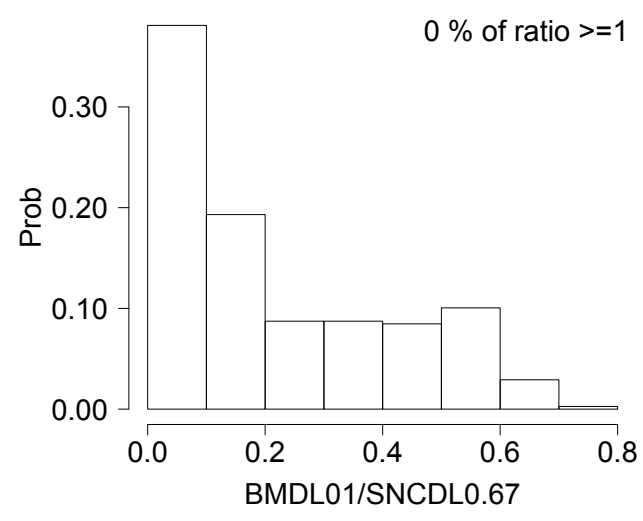

(b) BMDL01/bcSNCDL0.67

Figure 4.19: Histogram for Ratios of BMDL01 to Bias-corrected SNCDL

P95 and IQR for ratio of BMDL to bias corrected SNCDL. Figures 4.17, 4.18 and 4.19 includes the histograms, box plots and percentage of ratio greater than one for these ratios.

The medians for these six different ratios in table 4.10 are closer to one than medians for corresponding ratios in table 4.9 as expected. The skewness of the his- 
togram for these ratios remained right-skewed with bias correction. The percentage of ratios greater than one increased for the bias corrected ratio compared with ratio estimated using uncorrected specially for the one with SNCD0.67. We observe that median for ratio of BMDL10 to bias-corrected SNCDL0.67 is 0.971. Figure 4.17 shows that about half of bias-corrected SNCDL0.67 are smaller than BMDL10. Therefore, we can say that bias-corrected SNCDL0.67 is comparable to BMDL10.

\subsubsection{Extra Risk at SNCDL and SNCD}

By definition, benchmark dose is estimated for a fixed extra (or additional) risk which may be $0.1,0.05$ or 0.01 . However, neither the additional or extra risk is controlled in the estimation of SNCD. Having the knowledge of extra (or additional) risk corresponding to a $\mathrm{PoD}$ is useful in risk assessment. As a result, the corresponding extra risk at estimates of SNCD and SNCDL is calculated using Equation 4.3.

\subsubsection{Uncorrected}

\begin{tabular}{|l|l|l|l|l|l|}
\hline ExtraRisk & P05 & P25 & Median & P75 & P95 \\
\hline SNCDL1.0 & $6.36 \mathrm{e}-20$ & 0.002 & 0.070 & 0.126 & 0.236 \\
\hline SNCD1.0 & 0.093 & 0.178 & 0.210 & 0.339 & 0.517 \\
\hline SNCDU1.0 & 0.123 & 0.245 & 0.314 & 0.529 & 0.757 \\
\hline SNCDL0.67 & $9.16 \mathrm{e}-20$ & 0.001 & 0.038 & 0.065 & 0.145 \\
\hline SNCD0.67 & 0.032 & 0.084 & 0.103 & 0.158 & 0.335 \\
\hline SNCDU0.67 & 0.074 & 0.138 & 0.194 & 0.338 & 0.641 \\
\hline
\end{tabular}

Table 4.11: Quantiles for Risk of Uncorrected Signal-to-Noise Crossover Dose

Table 4.11 presents the quantiles of $05 \%, 25 \%, 50 \%, 75 \%$ and $95 \%$ for all extra risks corresponding to these uncorrected PoDs. We have seen previously that SNCD0.67 is comparable to BMD10. 90\% confidence interval for extra risk at SNCD0.67 is $(0.032,0.335)$ and the median is 0.103 . Panel $(\mathrm{b})$ in Figure 4.21 shows that about 


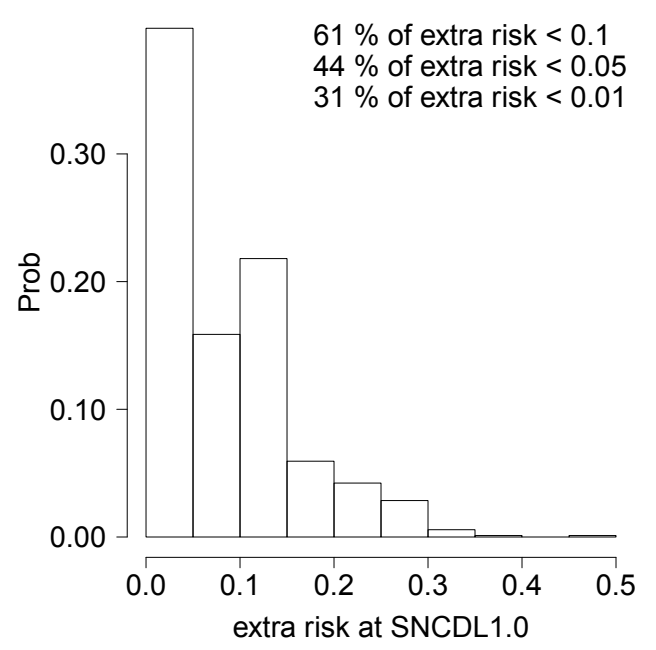

(a) SNCDL1.0

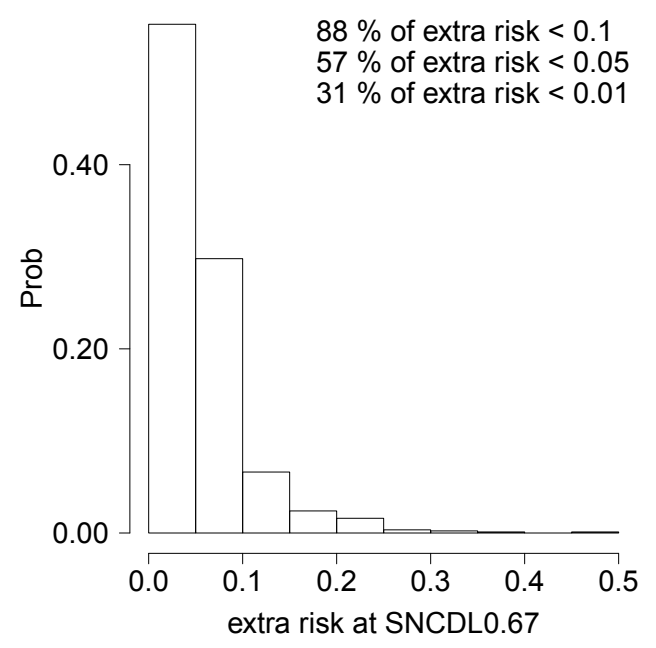

(b) SNCDL0.67

Figure 4.20: Histogram for Extra Risk at SNCDL

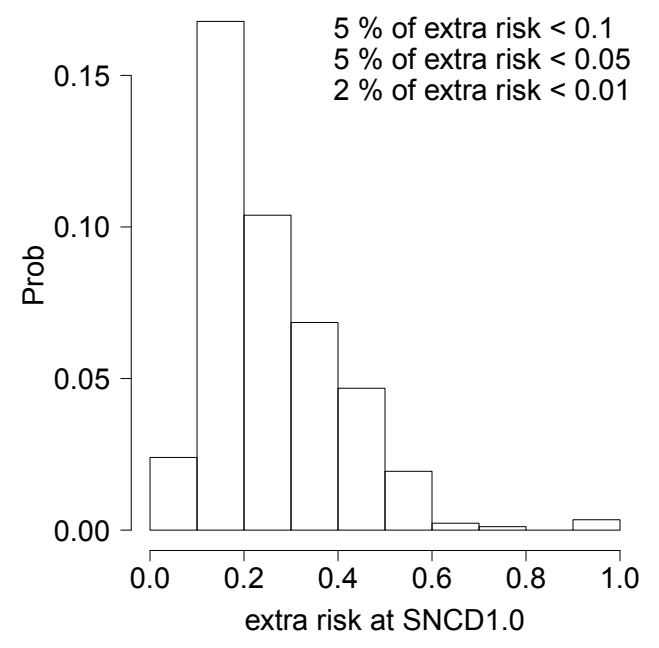

(a) SNCD1.0

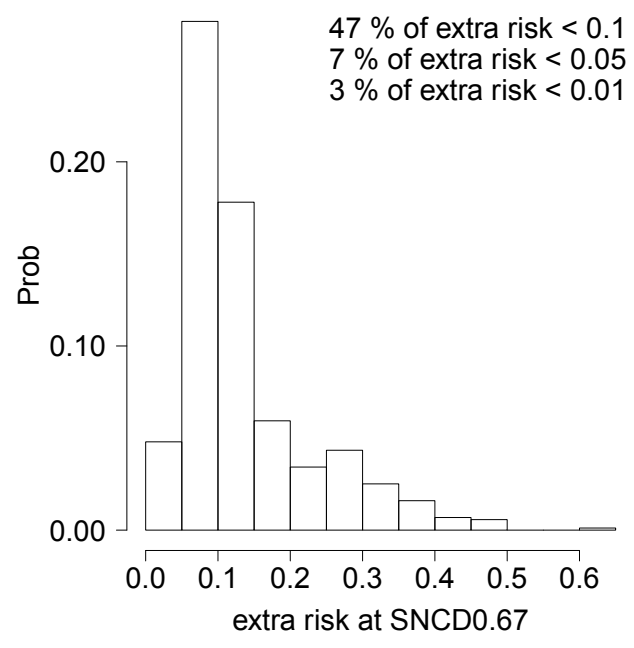

(b) SNCD0.67

Figure 4.21: Histogram for Extra Risk at SNCD

$47 \%$ of SNCD0.67 has extra risk smaller than 0.1 . We see that upper and lower quartile for SNCDL0.67 is $(0.001,0.065)$ and the median is 0.038. Further, panel (b) in Figure 4.20 shows that about $88 \%$ of SNCDL0.67 has extra risk less than 0.1 . These results confirm that based on the concept of risk, SNCD0.67 is comparable to BMD10 and SNCDL0.67 to BMDL10 even without bias correction. 


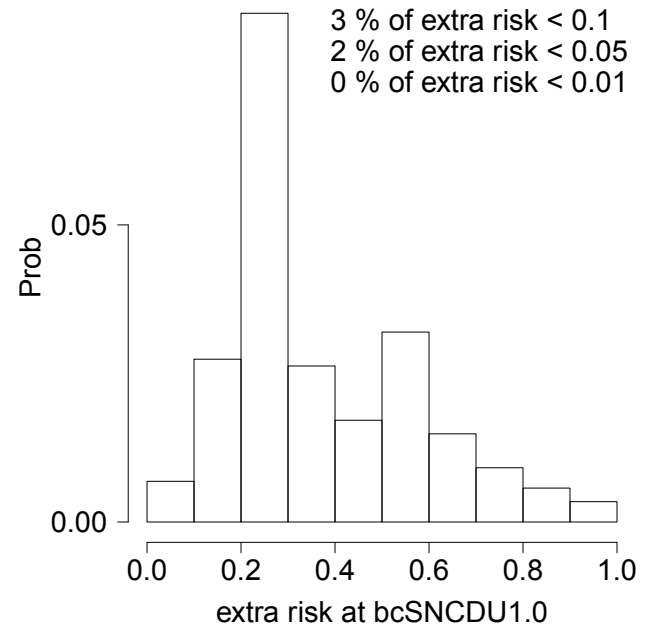

(a) SNCDU1.0

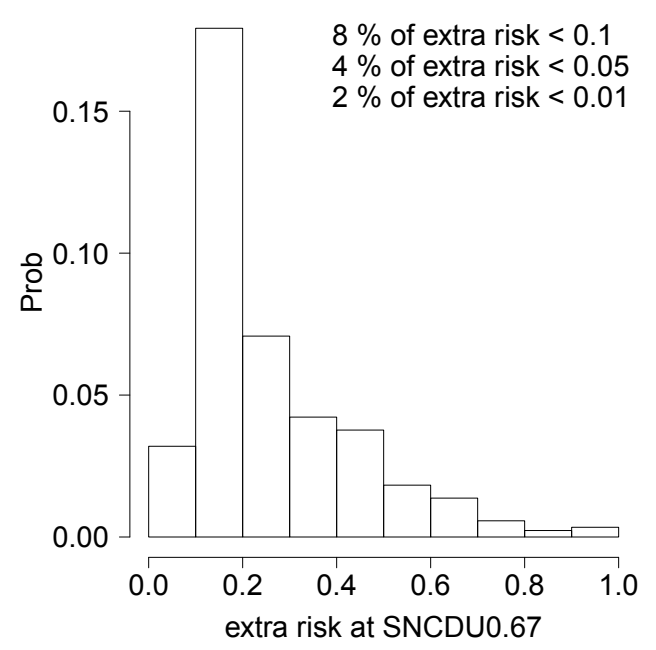

(b) SNCDU0.67

Figure 4.22: Histogram for Extra Risk at SNCDU

\subsubsection{Bias-corrected}

\begin{tabular}{|l|l|l|l|l|l|}
\hline ExtraRisk & P05 & P25 & Median & P75 & P95 \\
\hline bcSNCDL1.0 & $3.29 \mathrm{e}-21$ & 0.005 & 0.058 & 0.107 & 0.230 \\
\hline bcSNCD1.0 & 0.070 & 0.152 & 0.189 & 0.338 & 0.519 \\
\hline bcSNCDU1.0 & 0.160 & 0.229 & 0.291 & 0.519 & 0.755 \\
\hline bcSNCDL0.67 & $1.31 \mathrm{e}-4$ & 0.003 & 0.026 & 0.048 & 0.146 \\
\hline bcSNCD0.67 & 0.008 & 0.054 & 0.075 & 0.143 & 0.323 \\
\hline bcSNCDU0.67 & 0.051 & 0.116 & 0.171 & 0.351 & 0.649 \\
\hline
\end{tabular}

Table 4.12: Quantiles for Risk of Bias-corrected Signal-to-Noise Crossover Dose

All the results that are discussed in previous sections using uncorrected SNCDs improve with bias corrected SNCD. The lowest median extra risk reported is 0.026 for SNCDL0.67. The highest value of median reported for extra risk is 0.189 for bias corrected SNCD1.0. The percentages of extra risk that is less than 0.1 are $71 \%$ and 91\% for SNCDL1.0 and SNCDL0.67. These similar percentages of extra risk that are less than 0.05 and 0.01 are reported in the Figures 4.23 and 4.24. These percentages are reasonable for $\mathrm{SNCD}$ and $\mathrm{SNCDL}$ of $\mathrm{p}=2 / 3$. Based on these results, we can 


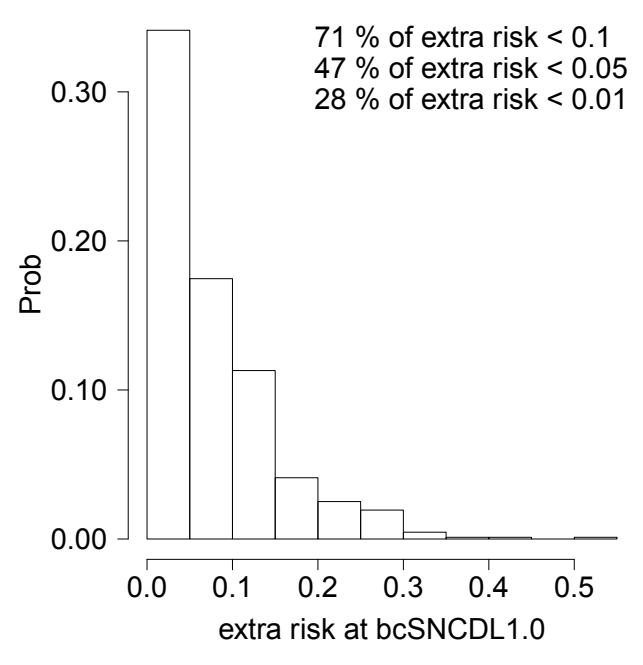

(a) bcSNCDL1.0

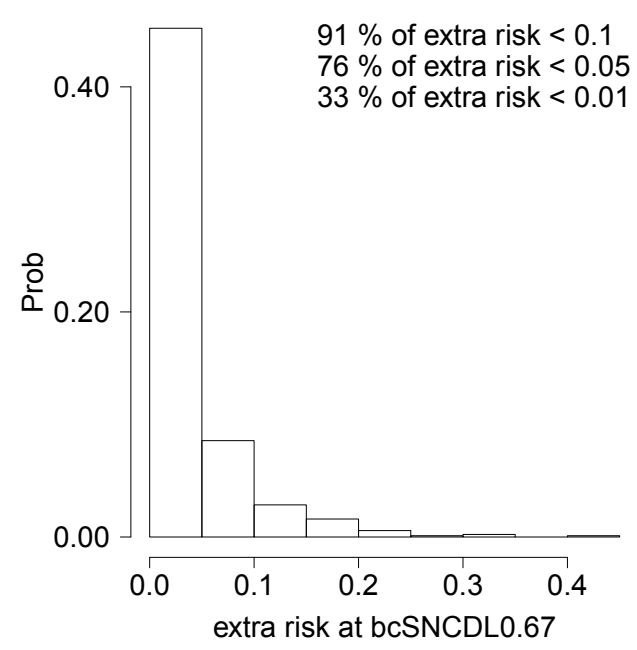

(b) bcSNCDL0.67

Figure 4.23: Histogram for Extra Risk at Bias-corrected SNCDL

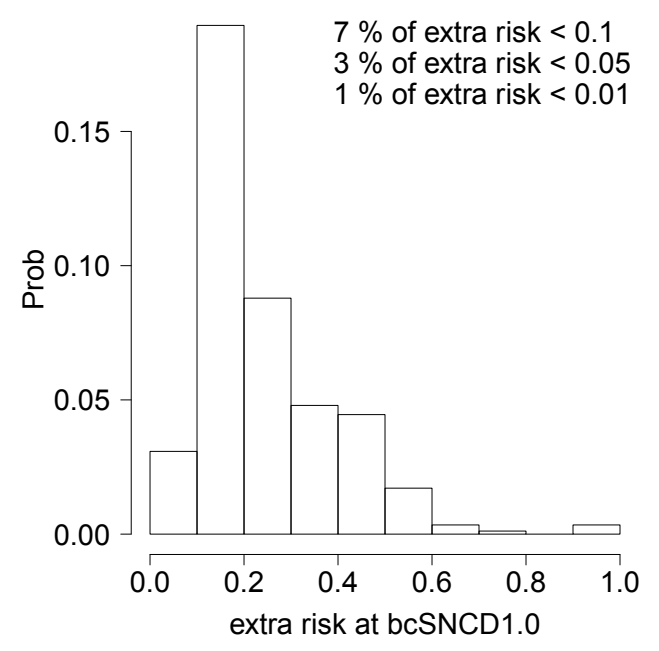

(a) bcSNCD1.0



(b) bcSNCD0.67

Figure 4.24: Histogram for Extra Risk at Bias-corrected SNCD

conclude that SNCD0.67 is comparable to BMD10. 


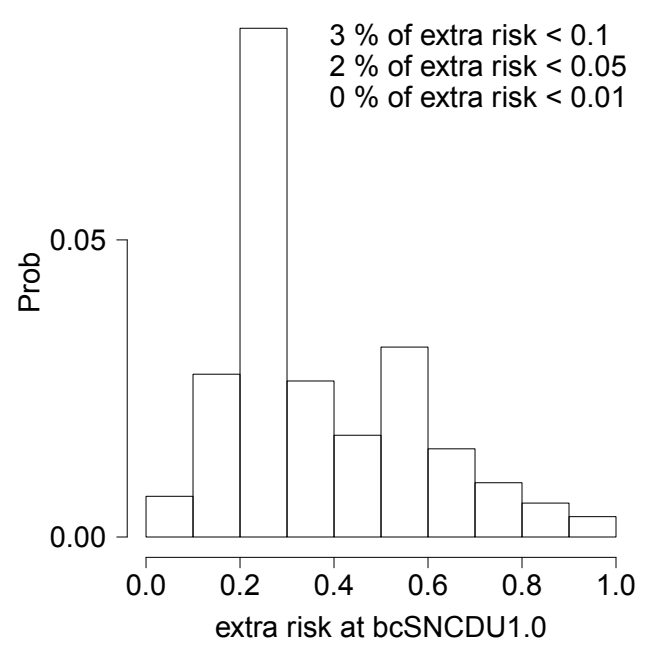

(a) bcSNCDU1.0

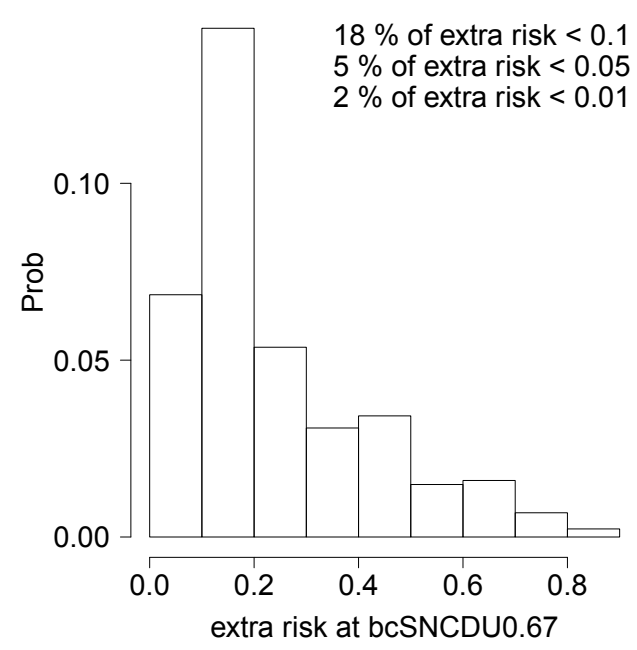

(b) bcSNCDU0.67

Figure 4.25: Histogram for Extra Risk at Bias-corrected SNCDU

\subsection{Summary and Conclusion}

Initially, the real data are fitted with Hill model using maximum likelihood approach. Datasets demonstrating significant dose-response trend and adequate goodnessof-fit are included in these analyses. Benchmark dose is estimated using theory developed in Chapter 2 and lower bound on BMD is estimated using traditional profile likelihood method. Estimates of signal-to-noise crossover dose is calculated using the theory developed in Chapter 3. Bias in these estimates are corrected using the multiple regression equation obtained in Chapter 3.

First, characteristics of SNCD and BMD are investigated using ratio of BMDL to BMD; and that of SNCDL to SNCD based on the results obtained from real datasets. Using simulation studies, we investigated the characteristics of the shape of histograms obtained for the ratio of SNCD to BMD by shape of the Hill model curves, number of dose levels and number of trials per dose. Further, to identify the signal-to-crossover dose and lower bound of SNCD that are comparable to the benchmark dose and lower bound of BMD, the ratio of BMD to SNCD and BMDL to 
SNCDL for all possible combination BMR (10\%, 5\% and 1\%) values and $\mathrm{p}(2 / 3$ and 1) of SNCD are investigated using results obtained from real datasets. Our analyzes showed that BMR of $10 \%$ (both point and lower bound) is comparable to SNR of $2 / 3$.

Figures 4.14 .2 and 4.3 show that variability in the ratio of BMDL to BMD is larger than that in the ratio of SNCDL to SNCD. This variability for this ratio decreases with decreasing BMR values. Confidence interval of SNCD is tighter than that of BMD as the median is higher for the former.

Our simulation studies show that shape of the histogram for the ratio of BMD to SNCD depends on the shape of the Hill model fitted, number of dose levels and number of trials per dose. The histograms are symmetric for the S-shaped and concave with initial slope greater than zero Hill curves with four doses and 50 trials per dose. For convex Hill model curve, the histograms tend toward symmetry when number of trials per dose is increased as opposed to that of number of dose levels. On the other hand, for concave with initial slope zero Hill model curve, similar behavior is observed when number of dose levels increased. Overall, there is an improvement in the symmetry whenever number of dose levels or number of trials per dose is increased.

When uncorrected and bias-corrected SNCD is compared with the BMD using results obtained from real data analyses for all possible combination of BMR values and p of SNCD, the results showed that SNCD0.67 is comparable to BMD10. Median for the ratio of BMD10 to uncorrected SNCD0.67 is 0.977 and that to bias-corrected is 1.058. About $43 \%$ of ratio of BMD10 to uncorrected SNCD0.67 and $63 \%$ that to bias-corrected SNCD0.67 are greater than one. These results confirm that half of the SNCD0.67 are smaller than BMD10. Median for the extra risk at uncorrected SNCD0.67 is 0.103 and that at bias-corrected is 0.075. It is clear that SNCD0.67 is comparable to BMD10.

We perform similar comparison as above for SNCDL with BMDL. Median for 
the ratio of BMDL10 to uncorrected SNCDL0.67 is reported as 0.897 and that to bias-corrected as 0.971. As the confidence interval of SNCD is tighter than that of $\mathrm{BMD}$, median for the ratio of BMDL to uncorrected SNCDL is smaller than one although the median for the ratio of BMD to uncorrected SNCD is close to one. However, we realize an improvement when bias-corrected. Median for extra risk at uncorrected SNCDL0.67 is 0.038 and that at bias-corrected is 0.026 . These values are much smaller than 0.1. Panel (b) in Figure 4.20 shows that $88 \%$ of uncorrected SNCDL0.67 and in Figure 4.23 shows that 91\% of bias-corrected SNCDL0.67 have extra risk smaller than 0.1 .

We observe an important drawback in the definition of signal-to-noise crossover dose. As the sample size (either number of trials per dose or number of dose levels) increases, the value of signal-to-noise crossover dose decreases. This implies that this measure as a point of departure could be zero for large enough sample. Implication of these results is that minimum allowable toxicity for a specific agent would be zero when estimated with large enough sample size.

In conclusion, these results suggests that SNCD0.67 is comparable to BMD10. In the literature, BMD10 has been used as a PoD when quantal animal data is used for analysis in risk assessment. Therefore, we can use SNCD0.67 or SNCDL0.67 in place of BMD10 or BMDL10. However, if BMR of $5 \%$ or $1 \%$ is needed, then there is a need to choose a smaller SNR value than that is investigated in this research. 


\section{BIBLIOGRAPHY}

[1] Auton.T.R. (1994) Calculation of Benchmark Doses from Teratology Data, Regulatory Toxicology and Pharmacology 19, 152-167 (1994).

[2] Bailer.A.J., Noble.R.B., and Wheeler.M.W. (2005) Model Uncertainity and Risk Estimation for Experimental Studies of Quantal Responses, Risk Analysis, Vol.25, No. 2, 2005.

[3] Crump,K., (1984) A new method for determining allowable daily intakes, Fundam Appl Toxicol 4:854-871.

[4] Crump,K.S., (1995) Calculation of benchmark dose from continuous data, Risk Analysis 15:79-89.

[5] Dourson.M.L., and Hertzberg.R.C. (1985) Novel Methods for the Estimation of Acceptable Daily Intake, Toxicology and Industrial Health, Vol. 1, No4, p23-41.

[6] Evans.J.S., Graham.J.D., Gray.G.M., and Sielken, Jr.R.L (1994) A Distributional Approach to Characterizing Low-Dose Cancer Risk, Risk Analysis, Vol.14, No.1, 1994

[7] Faustman.E.M., Allen.B.C., Kavlock.R.J., and Kimmel.C.A. (1994) Dose-Response Assessment for Developmental Toxicity - Characterization of Database and Determination of No Observed Adverse Effect Levels, Fundamental and Applied Toxicology 23, 478-486(1994)

[8] Faustman.E.M., Allen.B.C., Kavlock.R.J., and Kimmel.C.A. (1994) Dose-Response Assessment for Developmental Toxicity - Comparison of Generic Benchmark Dose Estimates with No Observed Adverse Effect Levels, Fundamental and Applied Toxicology 23, 487-495(1994) 
[9] Faustman.E.M., Allen.B.C., Kavlock.R.J., and Kimmel.C.A. (1994) Dose-Response Assessment for Developmental Toxicity - Statitical Models, Fundamental and Applied Toxicology 23, 496-509(1994)

[10] Faustman.E.M., Bartell.S.M. (1997) Review of noncancer risk assessment: applications of benchmark dose methods, Hum Ecol Risk Assess 3:893:920 (1997)

[11] Fowles.J.R., Alexeeff.G.V. and Dodge.D. (1999) The Use of Benchmark Dose Methodology with Acute Inhalation Lethality Data, Regulatory Toxicology and Phamacology 29, 262-278(1999)

[12] Fung.K.Y., Marro.L., and Krewiski.D. (1998) A Comparison of Methods for Estimating the Benchmark Dose Based on Overdispersed Data from Developmental Toxicity Studies, Risk Analysis Vol. 18, No.3, (1998)

[13] Gaylor.D.W., (1992) Incidence of developmental detects at the no observed adverse effect level (NOAEL). Regulatory Toxicology and Pharmacology $15: 151-160$

[14] Lehman,A.J., and Fitzhugh,O.G., (1954) 100-fold margin of safety, Assoc.Food Drug Off. U.S.Q. Bull. 18:33-35

[15] Moerbeek.M., Piersma.A.H., and Slob.W. (2004) A Comparison of Three Methods for Calculating Confidence Intervals for the Benchmark Dose, Risk Analysis, Vol. 24, No. 1, 2004

[16] Piegorsch.W.W., Xiong.H., Bhattacharya.R.N., and Lin.L. (2014) Benchmark Dose Analysis via Nonparametric Regression Modeling, Risk Analysis, Vol. 34, No. 1, 2014 
[17] Rai.K., and Van Ryzin.J. (1985) A Dose-Response Model for Teratological Experiments Involving Quantal Responses, Biometrics 41,1-9 (1985)

[18] Sand.S., Portier.C.J., and Krewski.D., (2011) A Signal-to-Noise Crossover Dose as the Point of Departure for Health Risk Assessment, Environ Health Perspect 119:1766-1774

[19] Schlosser.P.M., Lilly.P.D., Conolly.R.B., Janszen.D.B., and Kimbell.J.S. (2003) Benchmark dsoe risk assessment for formaldehyde using airflow modelling and single-compartment, DNA-protein cross-link dosimetry model to estimate human equivalent doses, Risk Analysis 23:473-487

[20] Shao.K., and Gift.J.S. (2014) Model uncertainty and Bayesian Model Averaged Benchmark Dose Estimation for continuous Data, Risk Analysis, Vol.34, No.1,2014

[21] Travis.K.Z., and Pate.I., and Welsh.Z.K. (2005) The role of the benchmark dose in a regulatory context, Regulatory Toxicology and Pharmacology $43(2006) 280-291$

[22] Zhu.Y., Wang.T., and Jelsovsky.J.Z.H. (2007) Bootstrap Estimation of Benchmark Doses and Confidence Limits with Clustered Quantal Data, Risk Analysis, Vol. 27, No. 2, 2007

[23] West.R.W., Nitcheva.D.K., and Piegorsch.W.W. (2009) Boostrap methods for simultaneous benchmark analysis with quantal response data, Environ Ecol Stat 16:63-73 (2009) 


\section{SUMMARY, CONCLUSION AND FUTURE WORK}

\subsection{Summary and Conclusion}

No-Observed-Adverse-Effect-Level (NOAEL) and benchmark dose (BMD) have been used as a PoD for human exposure guidelines. Bench mark dose has considerable merits compared to NOAEL as long as there are sufficient data, statistically significant trend, enough dose groups and dose-response relationships that is suitable for model fitting and estimates. Whenever, these requirements are satisfied, benchmark dose is recommended in the literature. However, if the estimation of BMD requires extrapolation beyond experimental dose range, then this estimation could be model dependent. Signal-to-noise crossover dose is introduced to overcome this limitation. Both BMD and SNCD estimation are similar till model fitting, but differs in the definition such that limitation found in BMD estimation can be overcome by SNCD. Previous work on SNCD focused on point estimation using NTP datasets.

In this research, chapter 3 simulation analysis is first carried out and extended to chapter 2. The chapter 3 analysis shows that improvement in the estimates of signal-to-noise crossover dose is significant when we increase the dose levels from four to six rather than from six to eight. These results motivate us to select only two dose levels which are four and six for chapter 2 studies.

In chapter 2, accuracy and precision of Hill model parameter is assessed. This investigation is performed on all possible combination of four different selected Hill model parameters, two different numbers of dose levels and four different numbers of trials per dose. The relative bias, that of standard deviation and coverage probability are reported for maximum likelihood and variance-covariance estimates of Hill model parameters. Similar results are reported for BMD based on extra risk and additional risk by applying linearization technique (Multi-variate Taylor Series expansion). Us- 
ing these results, the relationship between the maximum likelihood estimates of Hill model parameter and benchmark dose estimates and shape of the Hill curves, number of dose levels and number of trials per dose are investigated.

In chapter 3, we introduce analytical, parametric bootstrap and non-parametric bootstrap methods to obtain point and interval estimates of SNCD using simulation studies. Uncorrected and bias-corrected Coverage probabilities based on (SNCDL, SNCDU) and $\left(S N C D_{05}, S N C D_{95}\right)$ are estimated while using these three different approaches. While using analytical method, significant bias is observed in the estimation. Coverage probability is poor for analytical method without bias correction. However, after the bias correction, coverage probabilities are in the nominal range. Multiple regression equations to estimate the bias is derived as a function of the Hill model parameters, $\kappa$ and $\eta$, three different number of dose levels (four, six and eight) and six different number of trials per dose (50, 100, 200, 300, 400 and 500). Five different approaches to estimate the bias including the above mentioned multiple regression are introduced and compared to identify the best approach for bias estimation. Our simulation results show that multiple regression derived to estimate the bias is the best method. This equation is used to correct bias observed in SNCDs when SNCDs are estimated for the real datasets in chapter 4.

Hill model is fitted for all real datasets. Datasets demonstrating significant dose-response trend and adequate goodness of fit are included in the analyses. We apply the theory developed in Chapter 2 and 3 to this real data applications. Ratios of BMD to BMDL of BMR of 10\%, 05\% and 01\%, SNCD to SNCDL with and without bias correction of $\mathrm{p}=1$ and $2 / 3$ are calculated and their corresponding histograms and box plots are presented. Ratios of BMD to SNCD of all possible (six) combinatios with and without bias corrected SNCD are estimated and tables of quantile information, the corresponding histograms and box plots are included. These same results are presented for the ratios of BMDL to SNCDL of all possible 
combinations using uncorrected and bias corrected SNCDLs. Further, the extra risks associated with each of the estimates of SNCD and SNCDL (both uncorrected and bias corrected) are obtained and histograms are plotted with the percentage of extra risk that is less than 0.1, 0.05, and 0.01. These results show that SNCD0.67 is comparable to BMD10 and SNCDL0.67 is comparable to BMDL10.

\subsection{Future Work}

In Chapter 2, accuracy and precision of maximum likelihood and variancecovariance of Hill model parameters are investigated. We have found that the accuracy and precision of these estimates depended on the shape of the Hill model curves which is governed by combination of Hill model parameters. Having homogeneity of curves for certain values of Hill model parameters created instability in response probabilities. Consequently, this is reflected in the simulated values of the data and in the accuracy and precision of these estimates. It could be worth exploring to find a better estimation methods for the cases where accuracy and precision is low; or to identify a different dose-response model that reports higher accuracy and precision for shapes where the Hill model reports less accuracy and precision. The following dose-response models are previously used in the literature for quantal data: Logistic, Probit, Log-logistic, Log-probit, Weibull, Gamma and Linearized multistage family with one-stage or two-stage. It is possible that one of the above mentioned doseresponse models could give a better estimates where the Hill model fails. Identifying the location of dose points for different Hill curve shapes, number of dose levels and number of trials can be studied as well. Effect of background incident on the estimates of other Hill model parameters and BMD estimates can be further investigated.

The multiple regression equation obtained for bias correction is expressed as function of Hill model parameters $\kappa$ and $\eta$; different numbers of doses; and size per dose. This can be further extended to take account of other possible values, variable 
values of $\alpha$ and location of dose points. Chapter 2 results report that higher the value of $\alpha$, higher the homogeneity in $\kappa$ and $\eta$. Consequently, value of $\alpha$ could play significant role in the accuracy and precision of the estimates as the value of $\alpha$ increases accuracy and precision of other parameters decreases. Further, accuracy and precision of these estimates could be affected by whether or not the dose points are located on the higher curvature of the Hill curves.

In Chapter 3, the bias corrected coverage probability based on (SNCDL, SNCDU) does not fall within the nominal value. It could be worth exploring the theoretical reasons for this failure over the excellent outcomes for coverage probability based on $\left(S N C D_{0} 5, S N C D_{95}\right)$.

In Chapter 4, extra risks are reported for estimates of both uncorrected and bias corrected SNCD and SNCDL (SNCDU too). The percentage of these estimates with extra risk less than the usual BMR of $10 \%, 5 \%$, and $1 \%$ are reported. We report the extra risk at SNCDL of p of both 1 and 2/3. Estimation of extra risk at BMDL could be calculated and compared with extra risk at SNCDL. 


\section{A. APPENDIX OF CHAPTER 2}

\section{A.1 Distribution of Maximum Likelihood Estimates of Hill Model Parameters and BMD}

\section{A.1.1 Distribution of Hill Model Parameter Estimates}

Figure A.1 shows that normality assumption for maximum likelihood estimates of Hill model parameter $\kappa$ is satisfied when size per dose of 100 is used for convex curve. The assumption is satisfied for $\eta$ with 50. Concave with initial slope greater than zero behaves like S-shaped curve and normality assumption is satisfied for size per dose 50 with four doses (Figure A.2). On the other hand, for concave curve with initial slope zero, normality assumption for $\kappa$ is satisfied with four doses and size per dose of 50 , but for $\eta$, it is better satisfied with increasing the number of doses than that of size per dose (Figure A.3).

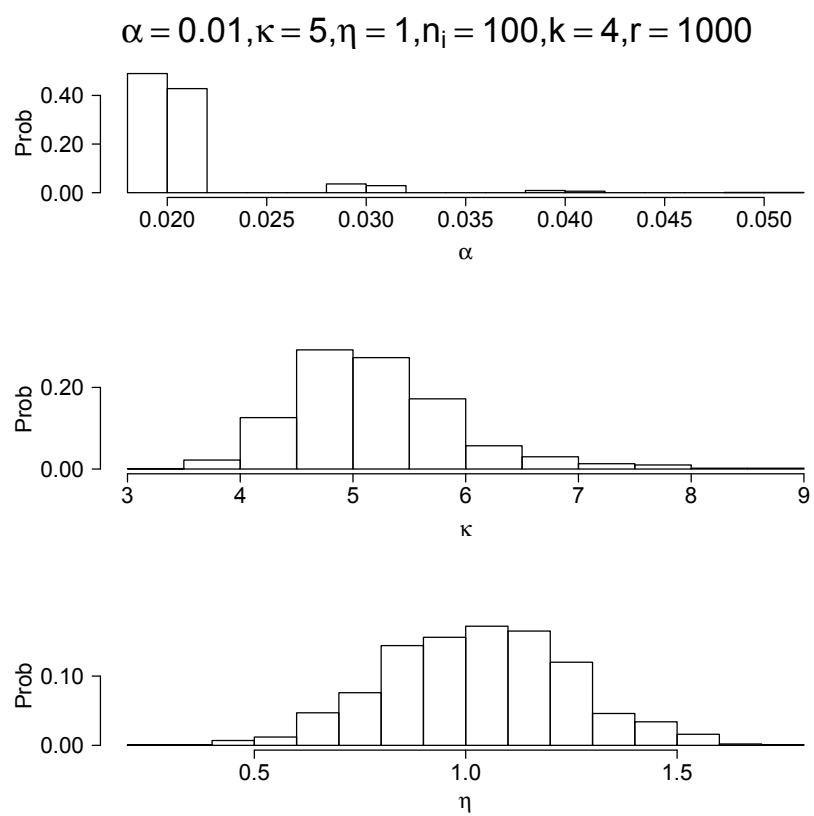

Figure A.1: Histogram for MLEs of Convex Hill Parameters when $n_{i}=50$ 

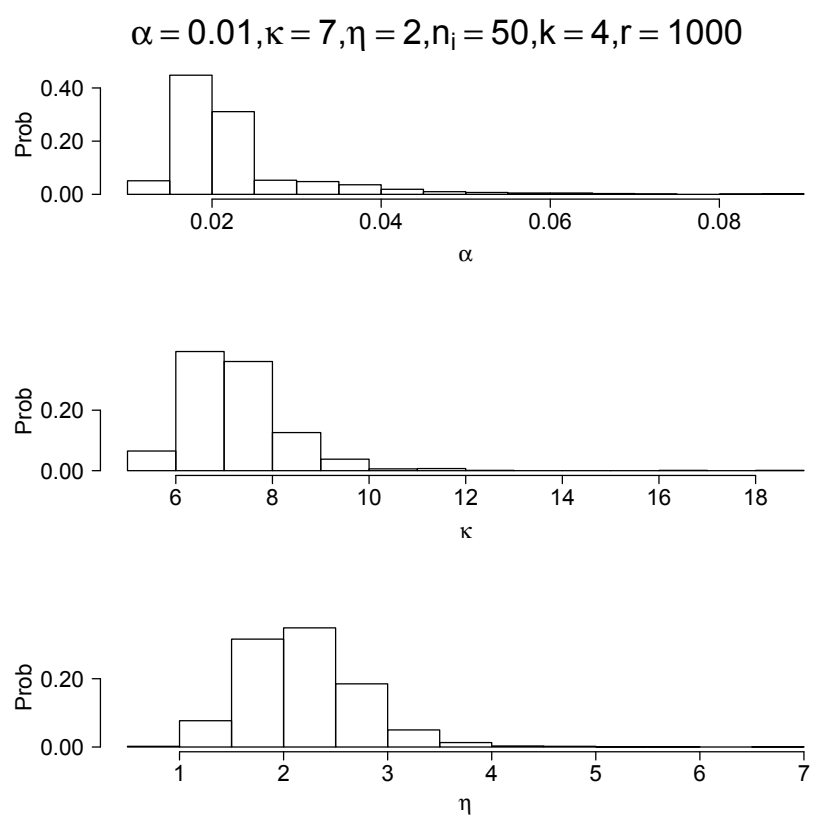

Figure A.2: Histogram for MLEs of Concave with initial slope $>0$ Hill Curve when $n_{i}=50$
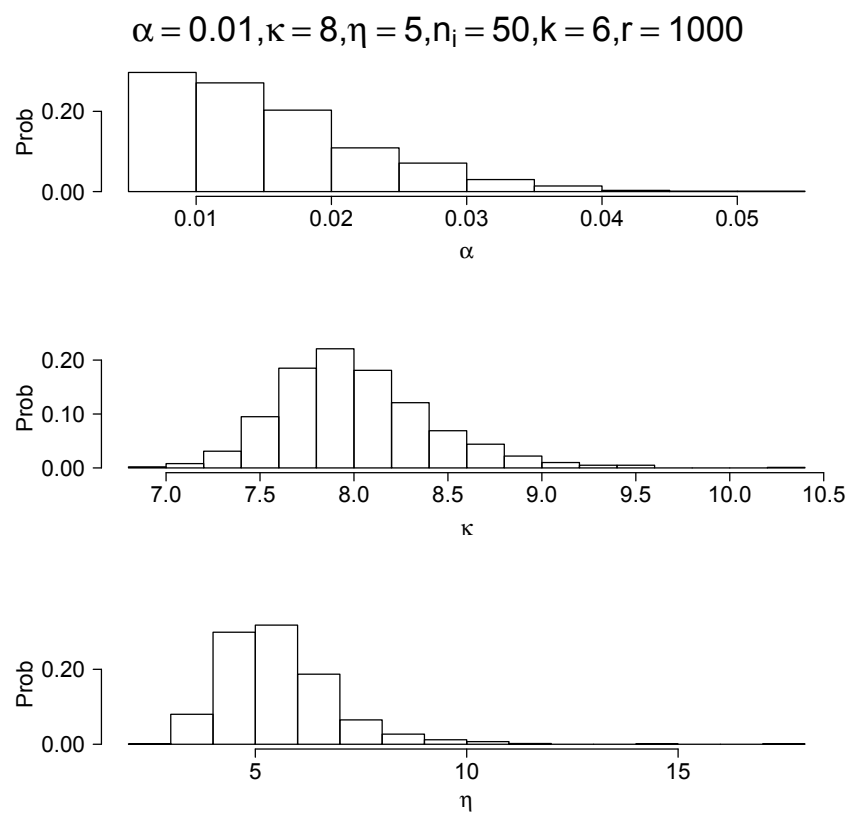

Figure A.3: Histogram for MLEs of Concave with initial slope $=0$ Hill Curve when $n_{i}=50$ 


\section{A.1.2 Distribution of BMD Estimates}

Normality assumption for benchmark dose estimates based on both extra and additional risk with BMR of $10 \%, 5 \%$ and $1 \%$ is satisfied at four doses and size per dose of 50 for S-shaped and concaver with initial slope greater than zero Hill curves (Figures 2.9 and A.6). Thugh normality assumption for Hill model parameter estimates are satisfied with size per dose of 100 for convex curve, the assumption is satisfied for BMD estimates of BMR of $10 \%$ and $5 \%$, but not for BMD estimates of BMR of $1 \%$ of which assumption is satisfied at size per dose of 500 . This assumption is better satisfied for concave curve with initial slope zero with six doses than size per dose just as expected (Figure A.7).
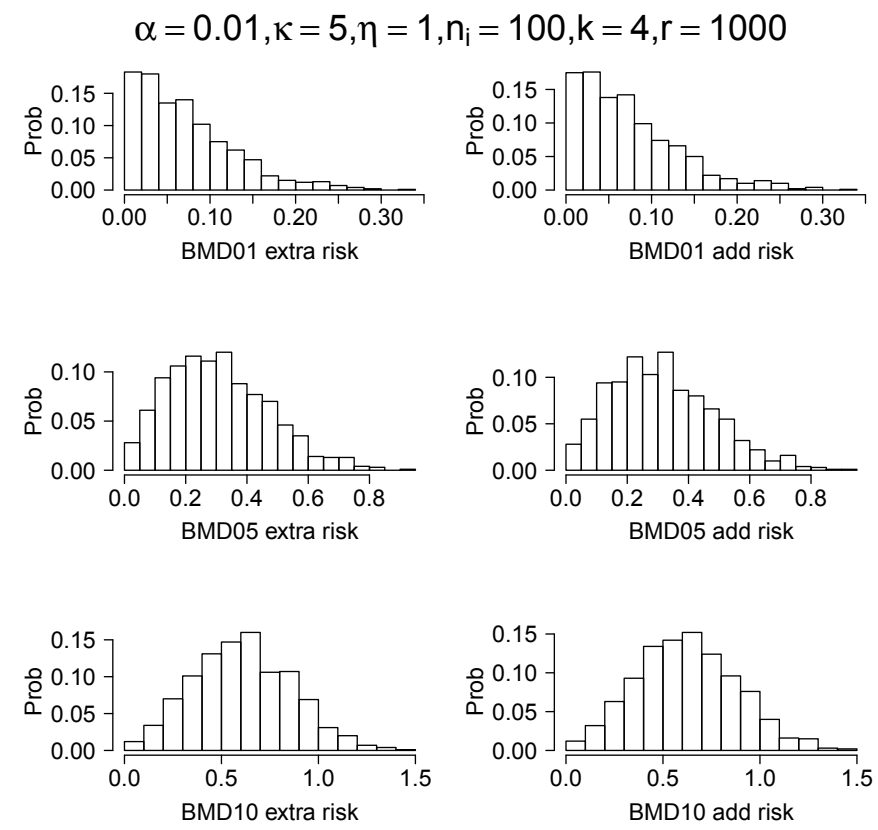

Figure A.4: Histogram for BMD Estimates on Convex Hill Curves when $n_{i}=100$ 

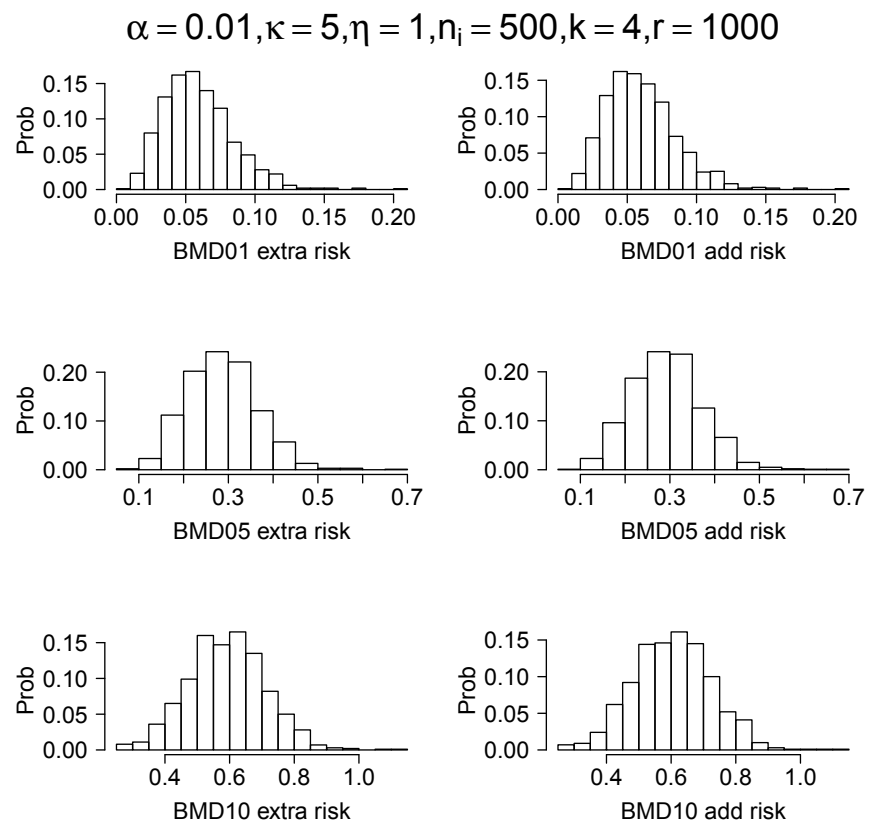

Figure A.5: Histogram for BMD Estimates on Convex Hill Curves when $n_{i}=500$
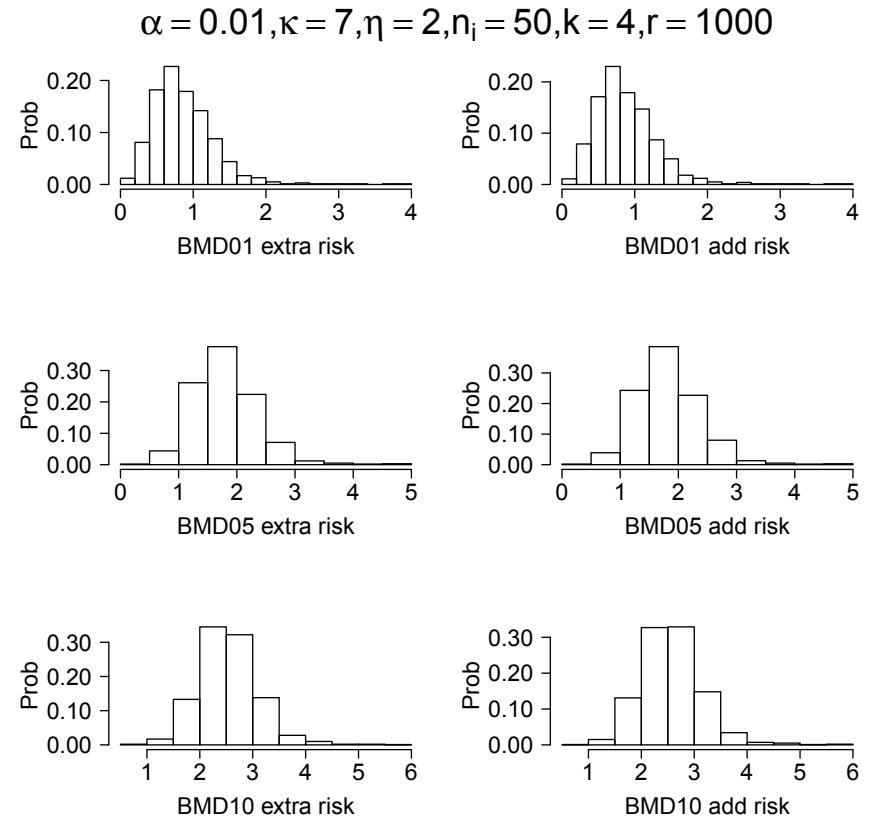

Figure A.6: Histogram for BMD Estimates on Concave with initial slope $>0$ Hill Curves 

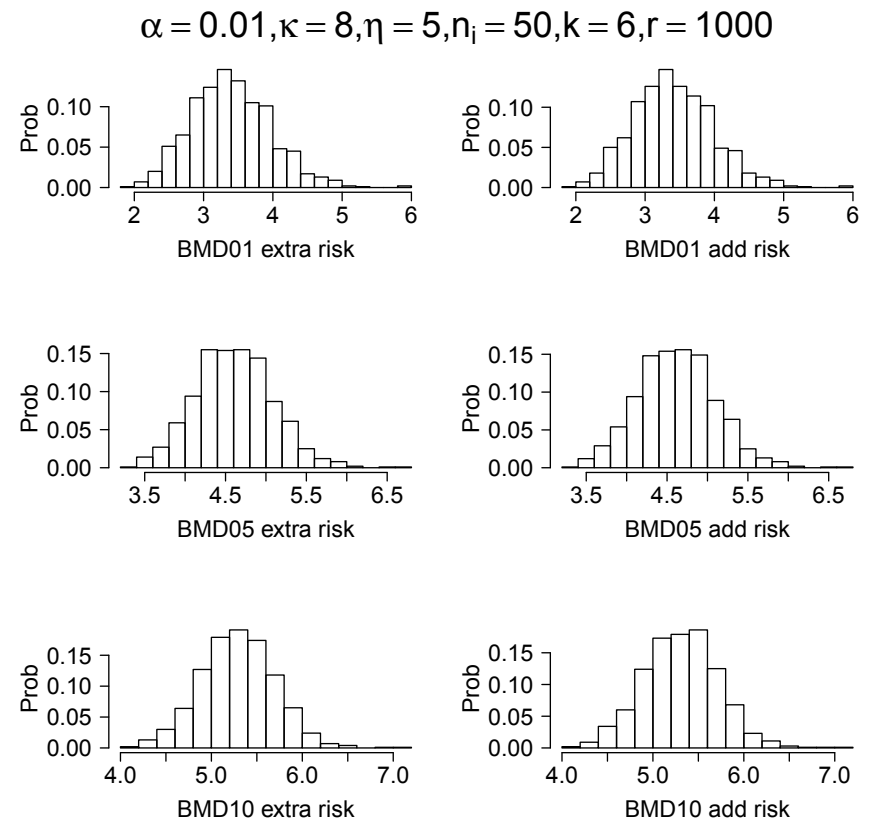

Figure A.7: Histogram for BMD Estimates on Concave with initial slope $=0$ Hill Curves

\section{A.2 Characteristics of Hill Model Curves for larger Background Inci- dent}

Figures A.8 and A.9 shows how homogeneity/heterogeneity of Hill model parameters $\kappa$ and $\eta$ change depending on the Hill model parameter values itself and the same phenomena for when background incident is changed from 0.01 to 0.5 (Compare Figures 2.2 with A.8 and 2.3 with A.9). Homogeneity of $\kappa$ and $\eta$ increases with increasing background incident. However, heterogeneity of $\kappa$ and $\eta$ is good enough to satisfy normality assumption of MLEs when $\alpha=0.5$ with S-shaped curve.

Figure A.10 shows that normality assumption of $\eta$ is violated at $\alpha=0.75$ for S-shaped curve. This assumption is satisfied for $\alpha$ with $\alpha=0.5$ at the expense of violating for both $\kappa$ and $\eta$ for convex curve (Figure A.11), concave with initial slope greater than zero (Figure A.12) and that with initial slope zero curve (Figure A.13). 


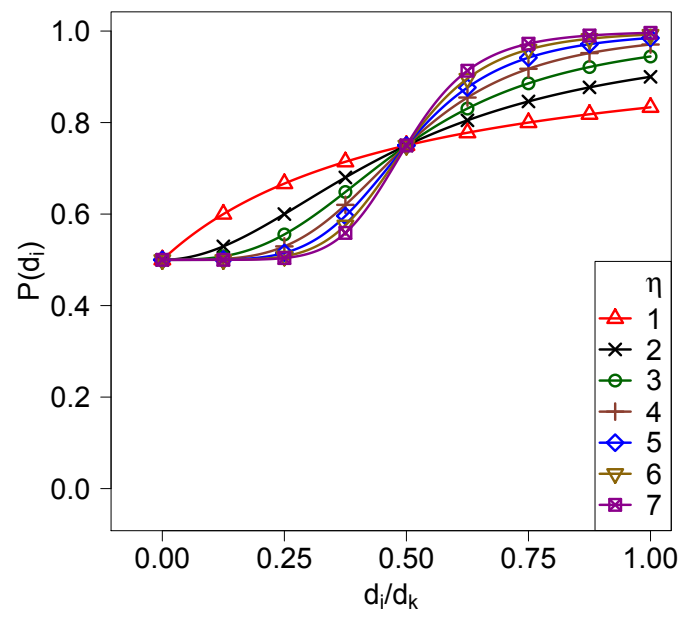

(a) $\kappa / d_{k}=0.500$

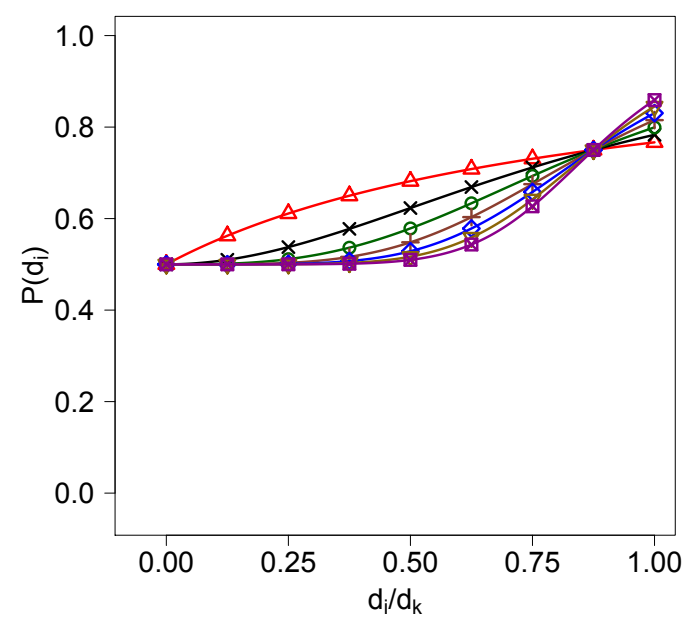

(c) $\kappa / d_{k}=0.875$

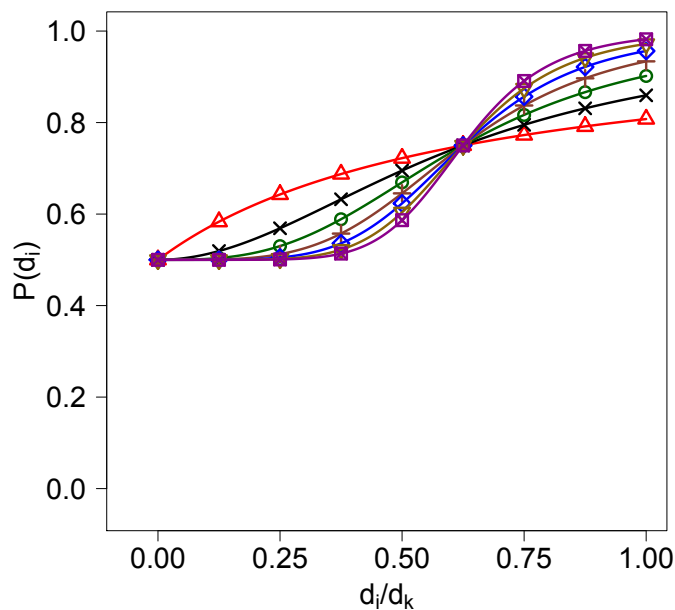

(b) $\kappa / d_{k}=0.625$

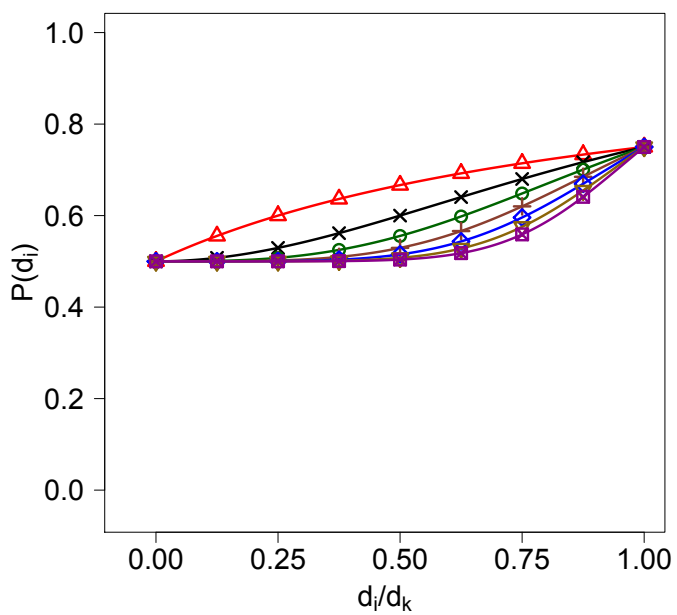

(d) $\kappa / d_{k}=1.000$

Figure A.8: Dose Response when $\eta=(1,2,3,5,5,7)$ with $\alpha=0.5$ 


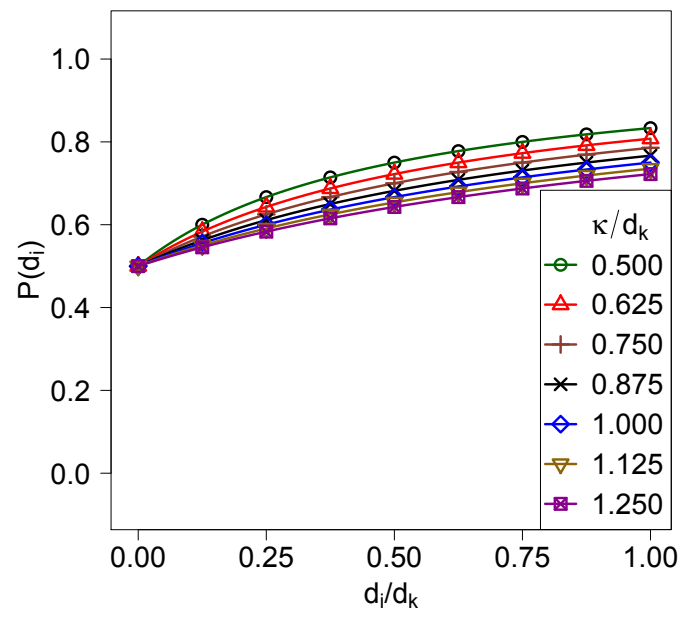

(a) $\eta=1$



(c) $\eta=3$



(b) $\eta=2$

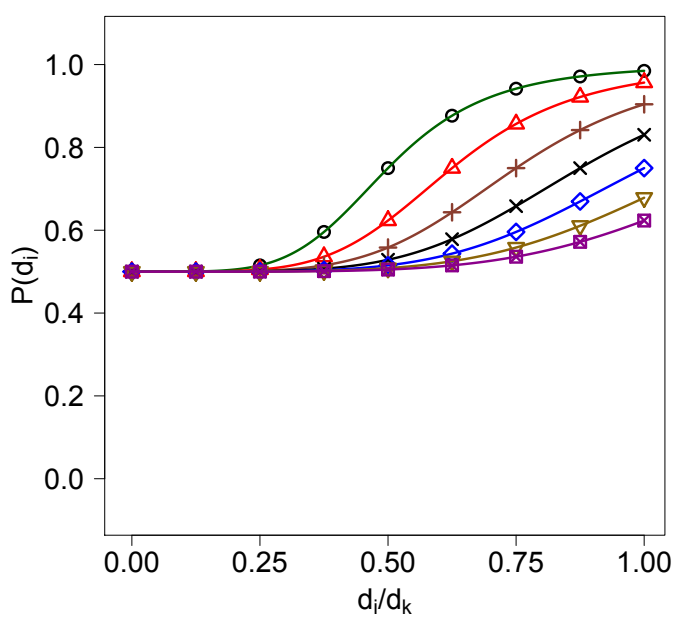

(d) $\eta=5$

Figure A.9: Dose Response when $\kappa / d_{k}=(0.5-1.2)$ with $\alpha=0.5$ 

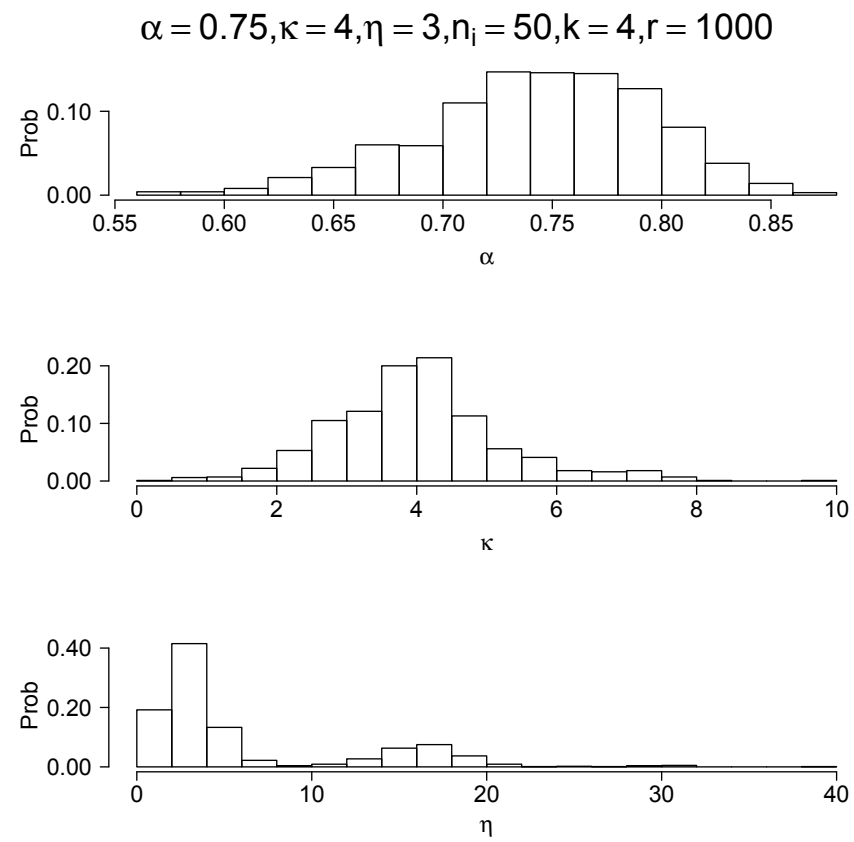

Figure A.10: Histogram for S-shaped Hill Curve Parameters when $\alpha=0.5$
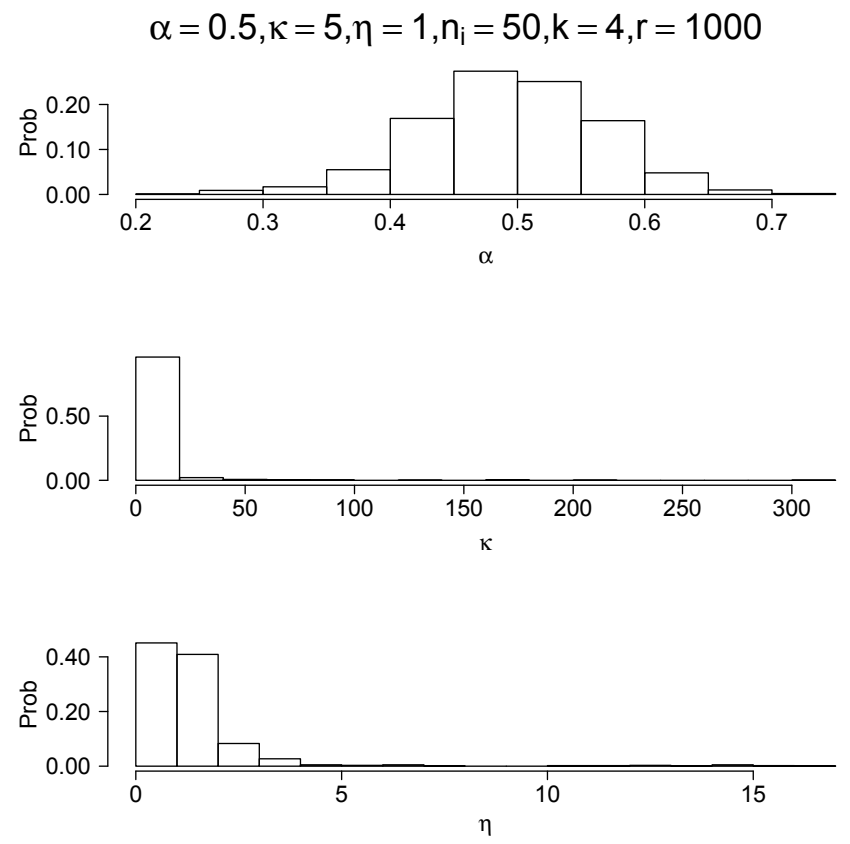

Figure A.11: Histogram for Convex Hill Curve Parameters when $\alpha=0.5$ 

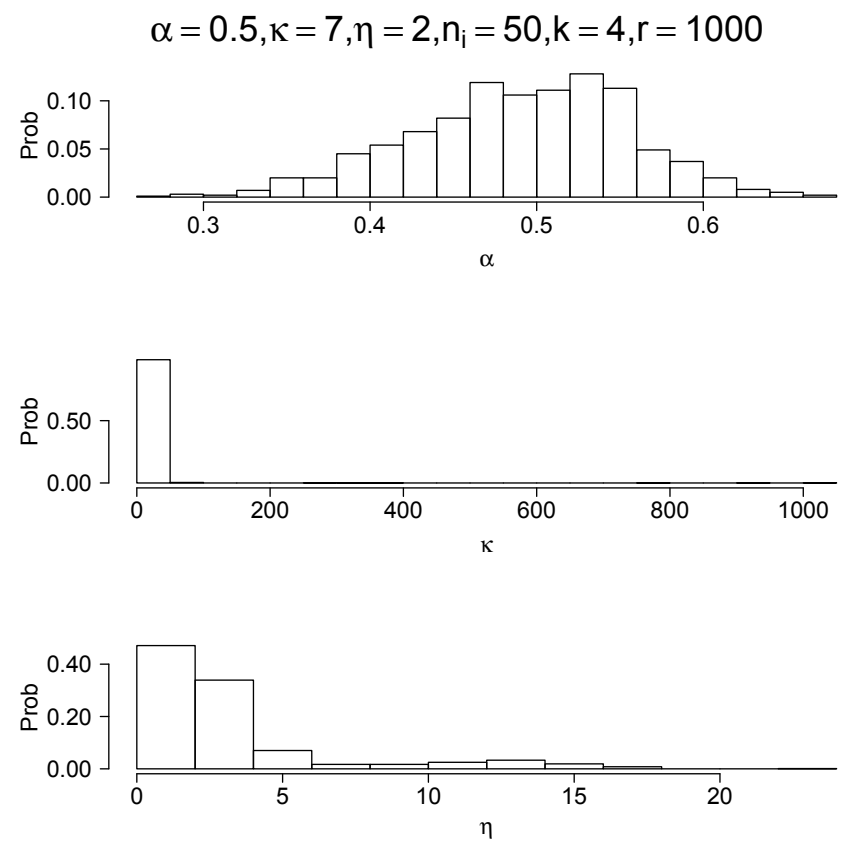

Figure A.12: Histogram for Concave with initial slope $>0$ Hill Curve Parameters when $\alpha=0.5$
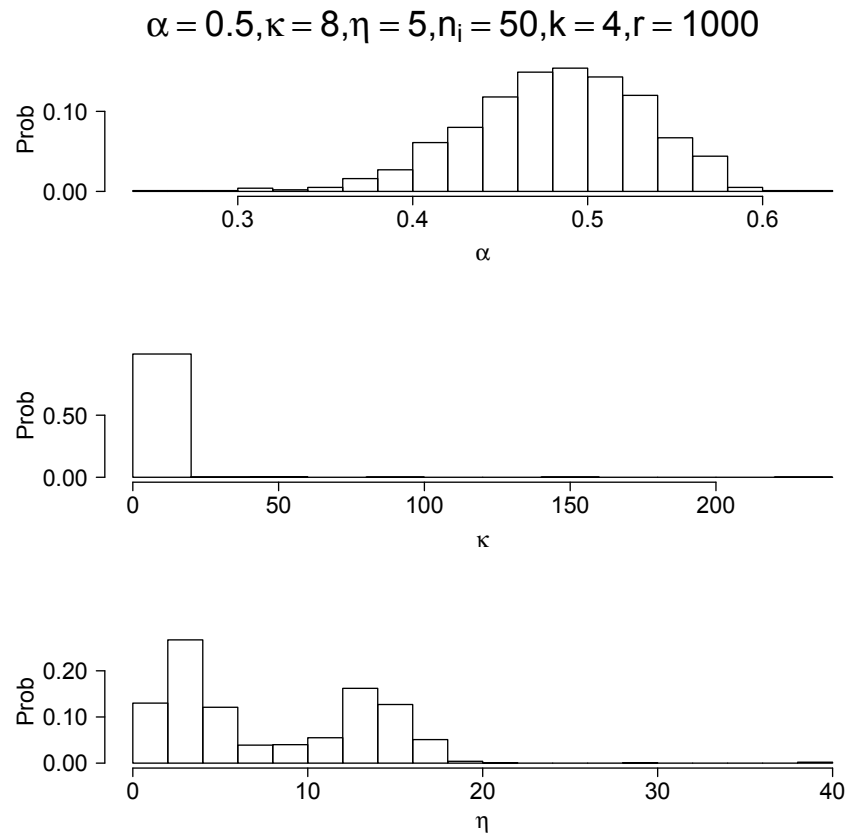

Figure A.13: Histogram for Concave with initial slope $=0$ Hill Curve Parameters when $\alpha=0.5$ 


\begin{tabular}{|c|c|c|c|c|c|c|}
\hline $\begin{array}{c}\text { Properites } \\
(\alpha, \kappa, \eta) \\
(0.1,4,3)\end{array}$ & $\begin{array}{c}\text { DoseGroups } \\
\left(d_{0}, \cdots, d_{t}\right) \\
0 \rightarrow 8 \mathrm{mg} / \mathrm{kg}\end{array}$ & $\begin{array}{c}\text { Size } / \\
\text { Dose Group } \\
n_{i}\end{array}$ & $\begin{array}{l}\text { Rel. } \\
\text { Bias }\end{array}$ & $\begin{array}{c}\text { Rel. } \\
\text { Stdev } \\
(\%)\end{array}$ & $\begin{array}{c}\text { Rel. } \\
\text { MSE } \\
(\%)\end{array}$ & $\begin{array}{c}\text { Conf./ } \\
\text { Est. } \\
(\%)\end{array}$ \\
\hline \multirow[t]{8}{*}{$\widehat{\alpha}$} & \multirow{4}{*}{$\begin{array}{l}(0,0.25 \\
0.5,1.0)\end{array}$} & 50 & -2.53 & 39.00 & 39.07 & 0.857 \\
\hline & & 100 & 0.98 & 28.53 & 28.54 & 0.880 \\
\hline & & 200 & 0.46 & 20.27 & 20.28 & 0.892 \\
\hline & & 500 & -0.64 & 12.84 & 12.86 & 0.891 \\
\hline & \multirow{4}{*}{$\begin{array}{c}(0,0.25, \\
0.375,0.5, \\
0.75,1.0)\end{array}$} & 50 & 0.54 & 39.64 & 39.64 & 0.866 \\
\hline & & 100 & 1.45 & 28.52 & 28.55 & 0.899 \\
\hline & & 200 & 0.59 & 20.23 & 20.24 & 0.890 \\
\hline & & 500 & -0.67 & 12.74 & 12.76 & 0.892 \\
\hline \multirow[t]{8}{*}{$\overline{\operatorname{Var}(\widehat{\alpha})}$} & \multirow{4}{*}{$\begin{array}{l}(0,0.25, \\
0.5,1.0)\end{array}$} & 50 & -3.59 & 35.20 & 35.39 & $\bar{~} 1.5 \mathrm{e}-3$ \\
\hline & & 100 & -9.50 & 23.63 & 25.47 & $8.1 \mathrm{e}-4$ \\
\hline & & 200 & -3.59 & 17.27 & 17.64 & $4.1 \mathrm{e}-4$ \\
\hline & & 500 & 1.61 & 11.04 & 11.16 & $1.6 \mathrm{e}-4$ \\
\hline & \multirow{4}{*}{$\begin{array}{c}(0,0.25, \\
0.375,0.5, \\
0.75,1.0)\end{array}$} & 50 & -2.38 & 34.27 & 34.35 & $1.6 \mathrm{e}-3$ \\
\hline & & 100 & 4.76 & 24.89 & 25.34 & $8.1 \mathrm{e}-4$ \\
\hline & & 200 & -0.75 & 17.13 & 17.14 & $4.1 \mathrm{e}-4$ \\
\hline & & 500 & -4.56 & 10.34 & 11.31 & $1.6 \mathrm{e}-4$ \\
\hline \multirow[t]{8}{*}{$\operatorname{Cov}(\alpha, \kappa)$} & \multirow{4}{*}{$\begin{array}{l}(0,0.25, \\
0.5,1.0)\end{array}$} & 50 & $\overline{0.17}$ & ב53.51 & ב53.51 & $77.0 \mathrm{e}-3$ \\
\hline & & 100 & -11.38 & 32.67 & 34.59 & $3.8 \mathrm{e}-3$ \\
\hline & & 200 & -1.65 & 24.38 & 24.44 & $1.9 \mathrm{e}-3$ \\
\hline & & 500 & 0.49 & 14.88 & 14.89 & $7.7 \mathrm{e}-4$ \\
\hline & \multirow{4}{*}{$\begin{array}{c}(0,0.25, \\
0.375,0.5, \\
0.75,1.0)\end{array}$} & 50 & -7.67 & 42.12 & 42.81 & $6.8 \mathrm{e}-3$ \\
\hline & & 100 & -1.847 & 30.87 & 30.92 & $3.5 \mathrm{e}-3$ \\
\hline & & 200 & 3.46 & 22.95 & 23.20 & $1.7 \mathrm{e}-3$ \\
\hline & & 500 & -7.27 & 12.79 & 14.71 & $6.9 \mathrm{e}-4$ \\
\hline \multirow[t]{8}{*}{$\overline{\operatorname{Cov}(\alpha, \eta)}$} & \multirow{4}{*}{$\begin{array}{l}(0,0.25, \\
0.5,1.0)\end{array}$} & 50 & -47.38 & 477.66 & 67.20 & $9.4 \mathrm{e}-3$ \\
\hline & & 100 & -15.32 & 51.15 & 53.40 & $4.9 \mathrm{e}-3$ \\
\hline & & 200 & 2.23 & 42.33 & 42.39 & $2.4 \mathrm{e}-3$ \\
\hline & & 500 & -1.99 & 23.13 & 23.22 & $9.1 \mathrm{e}-4$ \\
\hline & \multirow{4}{*}{$\begin{array}{c}(0,0.25, \\
0.375,0.5, \\
0.75,1.0)\end{array}$} & 50 & -6.86 & 68.25 & 68.59 & $8.3 \mathrm{e}-3$ \\
\hline & & 100 & 6.54 & 49.81 & 50.24 & $4.0 \mathrm{e}-3$ \\
\hline & & 200 & 6.37 & 34.88 & 35.46 & $2.0 \mathrm{e}-3$ \\
\hline & & 500 & -15.57 & 17.47 & 23.40 & $7.6 \mathrm{e}-4$ \\
\hline
\end{tabular}

Table A.1: Properties of $\alpha$ when $(\alpha, \kappa, \eta)=(0.1,4,3)$ 


\section{A.3 Tabulation Hill Model Parameter and BMD Estimates}

\section{A.3.1 Properties of Hill Model Parameter Estimates}

\begin{tabular}{|c|c|c|c|c|c|c|}
\hline $\begin{array}{c}\text { Dose-Respn } \\
\text { Shape } \\
\left(\alpha, \kappa / d_{t}, \eta\right) \\
\end{array}$ & $\begin{array}{c}\text { DoseGroups } \\
\left(d_{0}, \cdots, d_{t}\right) \\
0 \rightarrow 8 \mathrm{mg} / \mathrm{kg}\end{array}$ & $\begin{array}{c}\text { Size } / \\
\text { Dose Group } \\
n_{i}\end{array}$ & $\begin{array}{l}\text { Rel. } \\
\text { Bias } \\
(\%)\end{array}$ & $\begin{array}{c}\text { Rel. } \\
\text { Stdev } \\
(\%)\end{array}$ & $\begin{array}{l}\text { Rel. } \\
\text { MSE } \\
(\%)\end{array}$ & $\begin{array}{l}\text { Conf. } \\
\text { Cov. } \\
(\%)\end{array}$ \\
\hline \multirow{8}{*}{$\begin{array}{c}\text { S-shaped } \\
(0.01,0.500,3)\end{array}$} & \multirow{4}{*}{$\begin{array}{l}(0,0.25, \\
0.5,1.0)\end{array}$} & $\overline{50}$ & $\overline{1.94}$ & 7.80 & 8.04 & 0.916 \\
\hline & & 100 & 1.15 & 5.42 & 5.54 & 0.922 \\
\hline & & 200 & 1.18 & 3.81 & 3.99 & 0.899 \\
\hline & & 500 & 1.19 & 2.40 & 2.69 & 0.886 \\
\hline & \multirow{4}{*}{$\begin{array}{c}(0,0.25, \\
0.375,0.5,\end{array}$} & 50 & 1.29 & 5.80 & 5.94 & 0.916 \\
\hline & & 100 & 1.35 & 4.06 & 4.28 & 0.910 \\
\hline & & 200 & 0.96 & 2.84 & 2.99 & 0.913 \\
\hline & & 500 & 0.94 & 1.79 & 2.02 & 0.871 \\
\hline \multirow{8}{*}{$\begin{array}{c}\text { Convex } \\
(0.01,0.625,1)\end{array}$} & \multirow{4}{*}{$\begin{array}{l}(0,0.25 \\
0.5,1.0)\end{array}$} & 50 & 20.83 & 56.01 & 59.76 & $\overline{00.932}$ \\
\hline & & 100 & 4.67 & 19.54 & 20.09 & 0.943 \\
\hline & & 200 & 2.86 & 10.50 & 10.88 & 0.928 \\
\hline & & 500 & 2.79 & 6.34 & 6.93 & 0.918 \\
\hline & \multirow{4}{*}{$\begin{array}{c}(0,0.25, \\
0.375,0.5 \\
0.75,1.0)\end{array}$} & 50 & 6.33 & 27.65 & 28.37 & 0.954 \\
\hline & & 100 & 4.10 & 13.24 & 13.86 & 0.940 \\
\hline & & 200 & 2.49 & 8.05 & 8.42 & 0.928 \\
\hline & & 500 & 2.30 & 4.89 & 5.40 & 0.910 \\
\hline \multirow{8}{*}{$\begin{array}{c}\text { Concave } \\
\left(>0^{\mathrm{a}}\right) \\
(0.01,0.875,2)\end{array}$} & \multirow{4}{*}{$\begin{array}{l}(0,0.25 \\
0.5,1.0)\end{array}$} & $\overline{50}$ & 3.41 & 15.08 & 15.46 & 0.928 \\
\hline & & 100 & 2.25 & 9.68 & 9.94 & 0.925 \\
\hline & & 200 & 1.40 & 6.34 & 6.50 & 0.906 \\
\hline & & 500 & 1.18 & 3.90 & 4.07 & 0.902 \\
\hline & \multirow{4}{*}{$\begin{array}{c}(0,0.25, \\
0.375,0.5 \\
0.75,1.0)\end{array}$} & 50 & 2.29 & 12.04 & 12.26 & 0.919 \\
\hline & & 100 & 1.76 & 7.62 & 7.82 & 0.930 \\
\hline & & 200 & 1.02 & 5.15 & 5.25 & 0.912 \\
\hline & & 500 & 0.95 & 3.19 & 3.33 & 0.931 \\
\hline \multirow{8}{*}{$\begin{array}{c}\text { Concave } \\
\left(=0^{\mathrm{a}}\right) \\
(0.01,1.000,5)\end{array}$} & \multirow{4}{*}{$\begin{array}{l}(0,0.25 \\
0.5,1.0)\end{array}$} & 50 & 0.71 & 6.82 & 6.85 & 0.931 \\
\hline & & 100 & 0.45 & 4.08 & 4.11 & 0.944 \\
\hline & & 200 & 0.15 & 2.76 & 2.76 & 0.945 \\
\hline & & 500 & 0.21 & 1.74 & 1.75 & 0.916 \\
\hline & \multirow{4}{*}{$\begin{array}{c}(0,0.25, \\
0.375,0.5 \\
0.75,1.0)\end{array}$} & 50 & 0.09 & 5.69 & 5.69 & 0.904 \\
\hline & & 100 & 0.15 & 3.89 & 3.89 & 0.897 \\
\hline & & 200 & 0.01 & 2.68 & 2.68 & 0.905 \\
\hline & & 500 & -0.08 & 1.66 & 1.67 & 0.890 \\
\hline
\end{tabular}

${ }^{\mathrm{a}}$ initial slope.

Table A.2: Properties of MLEs for $\kappa$ 


\begin{tabular}{|c|c|c|c|c|c|c|}
\hline $\begin{array}{c}\text { Dose-Respn } \\
\text { Shape } \\
\left(\alpha, \kappa / d_{t}, \eta\right)\end{array}$ & $\begin{array}{c}\text { Dose Groups } \\
\left(d_{0}, \cdots, d_{t}\right) \\
0 \rightarrow 8 \mathrm{mg} / \mathrm{kg}\end{array}$ & $\begin{array}{c}\text { Size } / \\
\text { Dose Group } \\
n_{i}\end{array}$ & $\begin{array}{l}\text { Rel. } \\
\text { Bias } \\
(\%)\end{array}$ & $\begin{array}{l}\text { Rel. } \\
\text { Stdev } \\
(\%)\end{array}$ & $\begin{array}{c}\text { Rel. } \\
\text { MSE } \\
(\%)\end{array}$ & $\begin{array}{l}\text { Conf. } \\
\text { Cov. } \\
(\%)\end{array}$ \\
\hline \multirow{8}{*}{$\begin{array}{c}\text { S-shaped } \\
(0.01,0.500,3)\end{array}$} & $(0,0.25$ & 50 & $\overline{7.73}$ & 19.64 & 21.11 & $\overline{00.938}$ \\
\hline & $0.5,1.0)$ & 100 & 3.45 & 12.42 & 12.89 & 0.929 \\
\hline & & 200 & 2.50 & 8.52 & 8.88 & 0.913 \\
\hline & & 500 & 1.97 & 5.32 & 5.68 & 0.896 \\
\hline & $(0,0.25$ & 50 & 4.17 & 14.49 & 15.07 & 0.916 \\
\hline & $0.375,0.5$ & 100 & 2.75 & 9.86 & 10.24 & 0.897 \\
\hline & $0.75,1.0)$ & 200 & 1.78 & 6.82 & 7.05 & 0.895 \\
\hline & & 500 & 1.63 & 4.30 & 4.59 & 0.888 \\
\hline \multirow{8}{*}{$\begin{array}{c}\text { Convex } \\
(0.01,0.625,1)\end{array}$} & $(0,0.25$ & 50 & 3.86 & 32.49 & 32.71 & 0.910 \\
\hline & $0.5,1.0)$ & 100 & 2.55 & 22.73 & 22.88 & 0.891 \\
\hline & & 200 & 2.19 & 15.96 & 16.11 & 0.893 \\
\hline & & 500 & 1.05 & 10.07 & 10.12 & 0.913 \\
\hline & $(0,0.25$ & 50 & 2.63 & 28.55 & 28.68 & 0.914 \\
\hline & 0.375, 0.5, & 100 & 1.76 & 20.06 & 20.14 & 0.910 \\
\hline & $0.75,1.0)$ & 200 & 2.39 & 14.12 & 14.32 & 0.892 \\
\hline & & 500 & 1.69 & 8.91 & 9.07 & 0.896 \\
\hline \multirow{8}{*}{$\begin{array}{c}\text { Concave } \\
(>0) \\
(0.01,0.875,2)\end{array}$} & $(0,0.25$, & 50 & 10.01 & 30.59 & 32.18 & 0.928 \\
\hline & $0.5,1.0)$ & 100 & 5.45 & 17.49 & 18.32 & 0.929 \\
\hline & & 200 & 4.14 & 11.93 & 12.63 & 0.911 \\
\hline & & 500 & 3.69 & 7.45 & 8.31 & 0.888 \\
\hline & $(0,0.25$ & 50 & 7.86 & 23.82 & 25.09 & 0.919 \\
\hline & $0.375,0.5$ & 100 & 4.74 & 14.88 & 15.61 & 0.924 \\
\hline & $0.75,1.0)$ & 200 & 3.57 & 10.16 & 10.77 & 0.921 \\
\hline & & 500 & 3.32 & 6.39 & 7.20 & 0.894 \\
\hline \multirow{8}{*}{$\begin{array}{c}\text { Concave } \\
(=0) \\
(0.01,1.000,5)\end{array}$} & $(0,0.25$ & 50 & 62.26 & 271.00 & 278.06 & 0.774 \\
\hline & $0.5,1.0)$ & 100 & 35.89 & 149.14 & 153.40 & 0.851 \\
\hline & & 200 & 18.27 & 63.62 & 66.19 & 0.945 \\
\hline & & 500 & 8.37 & 16.40 & 18.41 & 0.989 \\
\hline & $(0,0.25$ & 50 & 12.05 & 28.82 & 31.23 & 0.926 \\
\hline & $0.375,0.5$ & 100 & 8.03 & 18.87 & 20.51 & 0.907 \\
\hline & $0.75,1.0)$ & 200 & 5.11 & 12.84 & 13.82 & 0.915 \\
\hline & & 500 & 4.79 & 8.00 & 9.32 & 0.894 \\
\hline
\end{tabular}

initial slope.

Table A.3: Properties of MLEs for $\eta$ 


\begin{tabular}{|c|c|c|c|c|c|c|}
\hline $\begin{array}{c}\text { Dose-Respn } \\
\text { Shape } \\
\left(\alpha, \kappa / d_{t}, \eta\right)\end{array}$ & $\begin{array}{c}\text { Dose Groups } \\
\left(d_{0}, \cdots, d_{t}\right) \\
0 \rightarrow 8 \mathrm{mg} / \mathrm{kg}\end{array}$ & $\begin{array}{c}\text { Size } / \\
\text { Dose Group } \\
n_{i}\end{array}$ & $\begin{array}{l}\text { Rel. } \\
\text { Bias } \\
(\%)\end{array}$ & $\begin{array}{c}\text { Rel. } \\
\text { Stdev } \\
(\%)\end{array}$ & $\begin{array}{l}\text { Rel. } \\
\text { MSE } \\
(\%)\end{array}$ & $\begin{array}{l}\text { Conf. } \\
\text { Cov. } \\
(\%)\end{array}$ \\
\hline \multirow{8}{*}{$\begin{array}{c}\text { S-shaped } \\
(0.01,0.500,3)\end{array}$} & \multirow{4}{*}{$\begin{array}{l}(0,0.25 \\
0.5,1.0)\end{array}$} & $\overline{\overline{50}}$ & 130 & 212 & 249 & 0.993 \\
\hline & & 100 & 109 & 142 & 179 & 0.995 \\
\hline & & 200 & 92.89 & 96.25 & 133 & 0.995 \\
\hline & & 500 & 96.15 & 61.22 & 114 & 0.721 \\
\hline & \multirow{4}{*}{$\begin{array}{c}(0,0.25, \\
0.375,0.5, \\
0.75,1.0)\end{array}$} & 50 & 123 & 209 & 243 & 0.999 \\
\hline & & 100 & 111 & 142 & 180 & 0.995 \\
\hline & & 200 & 92.28 & 95.68 & 133 & 0.988 \\
\hline & & 500 & 95.09 & 60.71 & 113 & 0.750 \\
\hline \multirow{8}{*}{$\begin{array}{c}\text { Convex } \\
(0.01,0.625,1)\end{array}$} & \multirow{4}{*}{$\begin{array}{l}(0,0.25 \\
0.5,1.0)\end{array}$} & 50 & 119 & 224 & 253 & 0.999 \\
\hline & & 100 & 113 & 144 & 182 & 0.993 \\
\hline & & 200 & 93.86 & 97.48 & 135 & 0.983 \\
\hline & & 500 & 96.78 & 62.09 & 115 & 0.985 \\
\hline & \multirow{4}{*}{$\begin{array}{c}(0,0.25, \\
0.375,0.5, \\
0.75,1.0)\end{array}$} & 50 & 117 & 141 & 184 & 0.999 \\
\hline & & 100 & 114 & 144 & 183 & 0.991 \\
\hline & & 200 & 95.08 & 97.77 & 136 & 0.981 \\
\hline & & 500 & 96.90 & 62.11 & 115 & 0.990 \\
\hline \multirow{8}{*}{$\begin{array}{c}\text { Concave } \\
(>0) \\
(0.01,0.875,2)\end{array}$} & \multirow{4}{*}{$\begin{array}{l}(0,0.25 \\
0.5,1.0)\end{array}$} & $\overline{50}$ & $\overline{128}$ & 210 & 246 & 0.989 \\
\hline & & 100 & 108 & 141 & 177 & 0.996 \\
\hline & & 200 & 92.24 & 95.62 & 133 & 0.994 \\
\hline & & 500 & 94.74 & 60.68 & 113 & 0.767 \\
\hline & \multirow{4}{*}{$\begin{array}{c}(0,0.25, \\
0.375,0.5 \\
0.75,1.0)\end{array}$} & 50 & 124 & 210 & 244 & 0.990 \\
\hline & & 100 & 107 & 141 & 177 & 0.996 \\
\hline & & 200 & 92.51 & 95.66 & 133 & 0.986 \\
\hline & & 500 & 94.76 & 60.64 & 113 & 0.733 \\
\hline \multirow{8}{*}{$\begin{array}{c}\text { Concave } \\
(=0) \\
(0.01,1.000,5)\end{array}$} & \multirow{4}{*}{$\begin{array}{l}(0,0.25 \\
0.5,1.0)\end{array}$} & 50 & 55.15 & 121 & 133 & 0.988 \\
\hline & & 100 & 60.09 & 88.91 & 107 & 0.974 \\
\hline & & 200 & 48.80 & 61.66 & 78.63 & 0.958 \\
\hline & & 500 & 52.03 & 39.70 & 65.45 & 0.776 \\
\hline & \multirow{4}{*}{$\begin{array}{c}(0,0.25, \\
0.375,0.5, \\
0.75,1.0)\end{array}$} & 50 & 51.96 & 107 & 119 & 0.971 \\
\hline & & 100 & 44.14 & 74.40 & 86.51 & 0.970 \\
\hline & & 200 & 40.79 & 52.46 & 66.46 & 0.935 \\
\hline & & 500 & 40.80 & 33.21 & 52.61 & 0.801 \\
\hline
\end{tabular}

initial slope.

Table A.4: Properties of MLEs for $\alpha$ 


\section{A.3.2 Properties of Hill Model Parameter Variance Estimates}

Tables summarize the relative bias, that of std, that of MSE and confidence coverage for variance of Hill model parameter estimates for four selected Hill Model parameter combination with changing number of doses and size per dose.

\begin{tabular}{|c|c|c|c|c|c|c|}
\hline $\begin{array}{c}\text { Dose-Respn } \\
\text { Shape } \\
\left(\alpha, \kappa / d_{t}, \eta\right)\end{array}$ & $\begin{array}{c}\text { Dose Groups } \\
\left(d_{0}, \cdots, d_{t}\right) \\
0 \rightarrow 8 \mathrm{mg} / \mathrm{kg}\end{array}$ & $\begin{array}{c}\text { Size } / \\
\text { Dose Group } \\
n_{i}\end{array}$ & $\begin{array}{l}\text { Var. } \\
\text { Est. } \\
(\%)\end{array}$ & $\begin{array}{l}\text { Rel. } \\
\text { Bias } \\
(\%)\end{array}$ & $\begin{array}{l}\text { Rel. } \\
\text { Stdev } \\
(\%)\end{array}$ & $\begin{array}{l}\text { Rel. } \\
\text { MSE } \\
(\%)\end{array}$ \\
\hline \multirow{8}{*}{$\begin{array}{c}\text { S-shaped } \\
(0.01,0.500,3)\end{array}$} & $(0,0.25$ & $\overline{50}$ & $9.74 \mathrm{e}-2$ & 11.18 & 31.89 & 33.79 \\
\hline & $0.5,1.0)$ & 100 & $4.71 \mathrm{e}-2$ & 12.21 & 19.63 & 23.12 \\
\hline & & 200 & $2.32 \mathrm{e}-2$ & 5.99 & 13.50 & 14.77 \\
\hline & & 500 & $9.28 \mathrm{e}-3$ & 12.34 & 8.93 & 15.23 \\
\hline & $(0,0.25$ & 50 & $5.38 \mathrm{e}-2$ & 16.57 & 26.36 & 31.14 \\
\hline & $0.375,0.5$ & 100 & $2.64 \mathrm{e}-2$ & 14.77 & 17.31 & 22.75 \\
\hline & $, 0.75,1.0)$ & 200 & $1.29 \mathrm{e}-2$ & 23.33 & 12.80 & 26.62 \\
\hline & & 500 & $5.13 \mathrm{e}-3$ & 15.59 & 7.30 & 17.21 \\
\hline \multirow{8}{*}{$\begin{array}{c}\text { Convex } \\
(0.01,0.625,1)\end{array}$} & $(0,0.25$ & 50 & 7.84 & -95.50 & 64.41 & 116 \\
\hline & $0.5,1.0)$ & 100 & 0.95 & 37.06 & 878.63 & 879.41 \\
\hline & & 200 & 0.28 & 12.16 & 75.35 & 76.33 \\
\hline & & 500 & 0.10 & 16.36 & 37.33 & 40.76 \\
\hline & $(0,0.25$ & 50 & 1.91 & 104 & 1450 & 1454 \\
\hline & $0.375,0.5$, & 100 & 0.44 & 15.36 & 222 & 223 \\
\hline & $0.75,1.0)$ & 200 & 0.16 & 6.72 & 61.54 & 61.91 \\
\hline & & 500 & $5.6 \mathrm{e}-2$ & 12.74 & 31.47 & 33.95 \\
\hline \multirow{8}{*}{$\begin{array}{c}\text { Concave } \\
(>0) \\
(0.01,0.875,2)\end{array}$} & $\overline{(0,0.25,}$ & 50 & 1.11 & 20.24 & 173 & 174 \\
\hline & $0.5,1.0)$ & 100 & 0.46 & -1.39 & 124 & 124 \\
\hline & & 200 & 0.20 & -4.11 & 42.26 & 42.46 \\
\hline & & 500 & $7.4 \mathrm{e}-2$ & 1.61 & 24.77 & 24.82 \\
\hline & $(0,0.25$ & 50 & 0.71 & 13.70 & 182 & 183 \\
\hline & $0.375,0.5$ & 100 & 0.28 & 10.51 & 66.55 & 67.37 \\
\hline & $0.75,1.0)$ & 200 & 0.13 & -1.40 & 37.80 & 37.83 \\
\hline & & 500 & $5.0 \mathrm{e}-2$ & 14.01 & 24.38 & 28.12 \\
\hline \multirow{8}{*}{$\begin{array}{c}\text { Concave } \\
(=0) \\
(0.01,1.000,5)\end{array}$} & $\overline{(0,0.25,}$ & $\overline{50}$ & $\overline{0.30}$ & 46.67 & 355 & 358 \\
\hline & $0.5,1.0)$ & 100 & 0.11 & 9.14 & 75.45 & 76.00 \\
\hline & & 200 & $4.86 \mathrm{e}-2$ & 13.65 & 47.44 & 49.37 \\
\hline & & 500 & $1.9 \mathrm{e}-2$ & 7.16 & 25.57 & 26.55 \\
\hline & $(0,0.25$ & 50 & 0.21 & 11.88 & 94.10 & 94.85 \\
\hline & $0.375,0.5$, & 100 & $9.7 \mathrm{e}-2$ & -0.75 & 58.38 & 58.39 \\
\hline & $0.75,1.0)$ & 200 & $4.6 \mathrm{e}-2$ & 7.81 & 37.49 & 38.30 \\
\hline & & 500 & $1.8 \mathrm{e}-2$ & 1.37 & 21.72 & 21.77 \\
\hline
\end{tabular}

initial slope.

Table A.5: Properties of Variance of MLE for $\kappa$ 


\begin{tabular}{|c|c|c|c|c|c|c|}
\hline $\begin{array}{c}\text { Dose-Respn } \\
\text { Shape } \\
\left(\alpha, \kappa / d_{t}, \eta\right)\end{array}$ & $\begin{array}{c}\text { Dose Groups } \\
\left(d_{0}, \cdots, d_{t}\right) \\
0 \rightarrow 8 \mathrm{mg} / \mathrm{kg}\end{array}$ & $\begin{array}{c}\text { Size } / \\
\text { Dose Group } \\
n_{i}\end{array}$ & $\begin{array}{l}\text { Var. } \\
\text { Est. } \\
(\%)\end{array}$ & $\begin{array}{l}\text { Rel. } \\
\text { Bias } \\
(\%)\end{array}$ & $\begin{array}{c}\text { Rel. } \\
\text { Stdev } \\
(\%)\end{array}$ & $\begin{array}{l}\text { Rel. } \\
\text { MSE } \\
(\%)\end{array}$ \\
\hline \multirow{8}{*}{$\begin{array}{c}\text { S-shaped } \\
(0.01,0.500,3)\end{array}$} & \multirow{4}{*}{$\begin{array}{l}(0,0.25, \\
0.5,1.0)\end{array}$} & $\overline{50}$ & 0.35 & $\overline{-62.44}$ & 36.29 & $\overline{772.22}$ \\
\hline & & 100 & 0.14 & 2.16 & 35.35 & 35.42 \\
\hline & & 200 & $6.5 \mathrm{e}-2$ & 5.13 & 22.87 & 23.44 \\
\hline & & 500 & $2.6 \mathrm{e}-2$ & 2.95 & 10.89 & 11.29 \\
\hline & \multirow{4}{*}{$\begin{array}{c}(0,0.25, \\
0.375,0.5, \\
0.75,1.0)\end{array}$} & 50 & 0.19 & 1.50 & 39.82 & 39.85 \\
\hline & & 100 & $8.7 \mathrm{e}-2$ & 3.52 & 21.05 & 21.34 \\
\hline & & 200 & $4.2 \mathrm{e}-2$ & -2.63 & 11.65 & 11.94 \\
\hline & & 500 & $1.7 \mathrm{e}-2$ & 3.73 & 7.23 & 8.14 \\
\hline \multirow{8}{*}{$\begin{array}{c}\text { Convex } \\
(0.01,0.625,1)\end{array}$} & \multirow{4}{*}{$\begin{array}{l}(0,0.25 \\
0.5,1.0)\end{array}$} & 50 & $\overline{0.11}$ & $\overline{0.21}$ & 12.55 & 12.55 \\
\hline & & 100 & $5.2 \mathrm{e}-2$ & -3.09 & 7.01 & 7.66 \\
\hline & & 200 & $2.5 \mathrm{e}-2$ & 1.06 & 4.77 & 4.88 \\
\hline & & 500 & $1 \mathrm{e}-2$ & 3.15 & 3.03 & 4.37 \\
\hline & \multirow{4}{*}{$\begin{array}{c}(0,0.25, \\
0.375,0.5, \\
0.75,1.0)\end{array}$} & 50 & $8.2 \mathrm{e}-2$ & 0.20 & 9.52 & 9.52 \\
\hline & & 100 & $4.0 \mathrm{e}-2$ & 4.32 & 5.65 & 7.11 \\
\hline & & 200 & $2.0 \mathrm{e}-2$ & -1.27 & 3.62 & 3.83 \\
\hline & & 500 & $7.9 \mathrm{e}-3$ & 1.20 & 2.27 & 2.57 \\
\hline \multirow{8}{*}{$\begin{array}{c}\text { Concave } \\
(>0) \\
(0.01,0.875,2)\end{array}$} & \multirow{4}{*}{$\begin{array}{l}(0,0.25, \\
0.5,1.0)\end{array}$} & $\overline{50}$ & 0.37 & 23.08 & 501 & 2502 \\
\hline & & 100 & 0.12 & 13.15 & 41.59 & 43.62 \\
\hline & & 200 & $5.7 \mathrm{e}-2$ & 8.15 & 25.86 & 27.11 \\
\hline & & 500 & $2.2 \mathrm{e}-2$ & 11.56 & 13.13 & 17.49 \\
\hline & \multirow{4}{*}{$\begin{array}{c}(0,0.25, \\
0.375,0.5, \\
0.75,1.0)\end{array}$} & 50 & 0.23 & -16.38 & 166 & 167 \\
\hline & & 100 & $8.9 \mathrm{e}-2$ & 17.59 & 45.14 & 48.44 \\
\hline & & 200 & 4.12 & 19.21 & 20.03 & 27.75 \\
\hline & & 500 & $1.6 \mathrm{e}-2$ & 19.32 & 11.00 & 22.23 \\
\hline \multirow{8}{*}{$\begin{array}{c}\text { Concave } \\
(=0) \\
(0.01,1.000,5)\end{array}$} & \multirow{4}{*}{$\begin{array}{l}(0,0.25, \\
0.5,1.0)\end{array}$} & 50 & 183.61 & 543.80 & 4238 & 4272 \\
\hline & & 100 & 55.61 & 272 & 2302 & 2319 \\
\hline & & 200 & 10.12 & 112 & 2347 & 2350 \\
\hline & & 500 & 0.67 & 28.76 & 709 & 710 \\
\hline & \multirow{4}{*}{$\begin{array}{l}(0,0.25, .375 \\
0.5,0.75,1.0)\end{array}$} & 50 & 2.10 & 10.38 & 102 & 102 \\
\hline & & 100 & 0.89 & 0.28 & 55.90 & 55.90 \\
\hline & & 200 & 0.41 & 15.82 & 47.31 & 49.88 \\
\hline & & 500 & 0.16 & 21.32 & 27.08 & 34.47 \\
\hline
\end{tabular}

initial slope.

Table A.6: Properties of Variance of MLE for $\eta$ 


\begin{tabular}{|c|c|c|c|c|c|c|}
\hline $\begin{array}{c}\text { Dose-Respn } \\
\text { Shape } \\
\left(\alpha, \kappa / d_{t}, \eta\right)\end{array}$ & $\begin{array}{c}\text { Dose Groups } \\
\left(d_{0}, \cdots, d_{t}\right) \\
0 \rightarrow 8 \mathrm{mg} / \mathrm{kg}\end{array}$ & $\begin{array}{c}\text { Size } / \\
\text { Dose Group } \\
n_{i}\end{array}$ & $\begin{array}{l}\text { Var. } \\
\text { Est. } \\
(\%)\end{array}$ & $\begin{array}{l}\text { Rel. } \\
\text { Bias } \\
(\%)\end{array}$ & $\begin{array}{l}\text { Rel. } \\
\text { Stdev } \\
(\%)\end{array}$ & $\begin{array}{l}\text { Rel. } \\
\text { MSE } \\
(\%)\end{array}$ \\
\hline \multirow{8}{*}{$\begin{array}{c}\text { S-shaped } \\
(0.01,0.500,3)\end{array}$} & \multirow{4}{*}{$\begin{array}{l}(0,0.25, \\
0.5,1.0)\end{array}$} & $\overline{50}$ & $\overline{4} 4.5 \mathrm{e}-4$ & 395 & $\overline{262}$ & 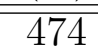 \\
\hline & & 100 & $2.0 \mathrm{e}-4$ & 1038 & 319 & 1085 \\
\hline & & 200 & $9.3 \mathrm{e}-5$ & 1067 & 246 & 1095 \\
\hline & & 500 & $3.8 \mathrm{e}-5$ & 1612 & 191 & 1623 \\
\hline & \multirow{4}{*}{$\begin{array}{c}0,0.25, \\
0.375,0.5, \\
0.75,1.0)\end{array}$} & 50 & $4.4 \mathrm{e}-4$ & 622 & 352 & 714 \\
\hline & & 100 & $2.0 \mathrm{e}-4$ & 817 & 275 & 862 \\
\hline & & 200 & $9.2 \mathrm{e}-5$ & 892 & 207 & 916 \\
\hline & & 500 & $3.7 e-5$ & 1831 & 215 & 1843 \\
\hline \multirow{8}{*}{$\begin{array}{c}\text { Convex } \\
(0.01,0.625,1)\end{array}$} & \multirow{4}{*}{$\begin{array}{l}(0,0.25 \\
0.5,1.0)\end{array}$} & 50 & $5.0 \mathrm{e}-4$ & 791 & 2806 & 2916 \\
\hline & & 100 & $2.1 \mathrm{e}-4$ & 836 & 201 & 860 \\
\hline & & 200 & $9.5 \mathrm{e}-5$ & 1014 & 165 & 1027 \\
\hline & & 500 & $3.9 \mathrm{e}-5$ & 3020 & 178 & 3025 \\
\hline & \multirow{4}{*}{$\begin{array}{c}(0,0.25, \\
0.375,0.5, \\
0.75,1.0)\end{array}$} & 50 & $2.0 \mathrm{e}-4$ & 111 & 8521 & 8522 \\
\hline & & 100 & $2.1 \mathrm{e}-4$ & 926 & 209 & 950 \\
\hline & & 200 & $9.6 \mathrm{e}-5$ & 1007 & 164 & 1020 \\
\hline & & 500 & $3.9 \mathrm{e}-5$ & 3022 & 178 & 3027 \\
\hline \multirow{8}{*}{$\begin{array}{c}\text { Concave } \\
(>0) \\
(0.01,0.875,2)\end{array}$} & \multirow{4}{*}{$\begin{array}{l}(0,0.25, \\
0.5,1.0)\end{array}$} & 50 & $4.4 \mathrm{e}-4$ & $\overline{383}$ & 269 & 469 \\
\hline & & 100 & $2.0 \mathrm{e}-4$ & 963 & 320 & 1015 \\
\hline & & 200 & $9.1 \mathrm{e}-5$ & 956 & 239 & 985 \\
\hline & & 500 & $3.7 e-5$ & 1761 & 214 & 1774 \\
\hline & \multirow{4}{*}{$\begin{array}{c}(0,0.25, \\
0.375,0.5, \\
0.75,1.0)\end{array}$} & 50 & $4.4 \mathrm{e}-4$ & 430 & 337 & 546 \\
\hline & & 100 & $2.0 \mathrm{e}-4$ & 909 & 336 & 969 \\
\hline & & 200 & $9.2 \mathrm{e}-5$ & 785 & 196 & 809 \\
\hline & & 500 & $3.7 e-5$ & 1431 & 184 & 1443 \\
\hline \multirow{8}{*}{$\begin{array}{c}\text { Concave } \\
(=0) \\
(0.01,1.000,5)\end{array}$} & \multirow{4}{*}{$\begin{array}{l}(0,0.25 \\
0.5,1.0)\end{array}$} & 50 & $1.5 \mathrm{e}-4$ & 172 & 137 & 220 \\
\hline & & 100 & $7.9 \mathrm{e}-5$ & 167 & 98 & 193 \\
\hline & & 200 & $3.8 \mathrm{e}-5$ & 173 & 69.51 & 186 \\
\hline & & 500 & $1.6 \mathrm{e}-5$ & 204 & 46.86 & 209 \\
\hline & \multirow{4}{*}{$\begin{array}{c}(0,0.25, \\
0.375,0.5, \\
0.75,1.0)\end{array}$} & 50 & $1.1 \mathrm{e}-4$ & 92.40 & 106 & 141 \\
\hline & & 100 & $5.5 \mathrm{e}-5$ & 126 & 89.42 & 155 \\
\hline & & 200 & $2.8 \mathrm{e}-5$ & 99.78 & 57.12 & 115 \\
\hline & & 500 & $1.1 \mathrm{e}-5$ & 116 & 39.82 & 123 \\
\hline
\end{tabular}

initial slope.

Table A.7: Properties of Variance of MLE for $\alpha$ 


\section{A.3.3 Properties of Hill Model Parameter Covariance Estimates}

The Tables summarize the relative bias, that of std, that of MSE of covariance between Hill model parameter estimates for four selected Hill Model parameter combination with changing number of doses and size per dose.

\begin{tabular}{|c|c|c|c|c|c|c|}
\hline $\begin{array}{c}\text { Dose-Respn } \\
\text { Shape } \\
\left(\alpha, \kappa / d_{t}, \eta\right)\end{array}$ & $\begin{array}{c}\text { Dose Groups } \\
\left(d_{0}, \cdots, d_{t}\right) \\
0 \rightarrow 8 \mathrm{mg} / \mathrm{kg}\end{array}$ & $\begin{array}{c}\text { Size/ } \\
\text { Dose Group }\end{array}$ & $\begin{array}{c}\text { Cov. } \\
\text { Est. } \\
(\%)\end{array}$ & $\begin{array}{c}\text { Rel. } \\
\text { Bias } \\
(\%)\end{array}$ & $\begin{array}{c}\text { Rel. } \\
\text { Stdev } \\
(\%)\end{array}$ & $\begin{array}{c}\text { Rel. } \\
\text { MSE } \\
(\%)\end{array}$ \\
\hline \hline S-shaped & $(0,0.25$, & 50 & $2.5 \mathrm{e}-2$ & 161 & 399 & 430 \\
$(0.01,0.500,3)$ & $0.5,1.0)$ & 100 & $9.5 \mathrm{e}-3$ & 182 & 194 & 266 \\
& & 200 & $4.1 \mathrm{e}-3$ & 135 & 124 & 183 \\
& & 500 & $1.6 \mathrm{e}-3$ & 265 & 85.74 & 279 \\
\cline { 2 - 7 } & $(0,0.25,$, & 50 & $1.7 \mathrm{e}-2$ & 12.44 & 105 & 106 \\
& 0.3750 .5$, & 100 & $7.0 \mathrm{e}-3$ & 87.74 & 96.35 & 130 \\
& $0.75,1.0)$ & 200 & $3.2 \mathrm{e}-3$ & 72.60 & 50.89 & 88.66 \\
& & 500 & $1.2 \mathrm{e}-3$ & 3.73 & 7.23 & 8.14 \\
\hline \hline Convex & $(0,0.25$, & 50 & -0.21 & -71.42 & -93.89 & 118 \\
$(0.01,0.625,1)$ & $0.5,1.0)$ & 100 & $-6.8 \mathrm{e}-2$ & -12.65 & -141 & 141 \\
& & 200 & $-2.7 \mathrm{e}-2$ & -12.65 & -58.97 & 60.31 \\
& & 500 & $-1.0 \mathrm{e}-2$ & 12.62 & -42.30 & 44.15 \\
\cline { 2 - 7 } & $(0,0.25$, & 50 & -0.11 & 11.20 & -210 & 211 \\
& $0.375,0.5$, & 100 & $-4.5 \mathrm{e}-2$ & -11.69 & -93.70 & 94.42 \\
& $0.75,1.0)$ & 200 & $-1.9 \mathrm{e}-2$ & -22.20 & -46.48 & 51.51 \\
& & 500 & $-7.1 \mathrm{e}-3$ & -11.11 & -29.90 & 31.89 \\
\hline \hline Concave & $(0,0.25$, & 50 & -0.21 & -11.61 & -88.98 & 89.73 \\
$(>0)$ & $0.5,1.0)$ & 100 & $-9.7 \mathrm{e}-2$ & -14.70 & -55.08 & 57.01 \\
& & 200 & $-4.5 \mathrm{e}-2$ & -12.69 & -33.39 & 35.72 \\
$(0.01,0.875,2)$ & & 500 & $-1.8 \mathrm{e}-2$ & -1.84 & -21.95 & 22.03 \\
\hline \hline Concave & $(0,0.25$, & 50 & -0.17 & -9.55 & -65.76 & 66.45 \\
$(0.01,1.000,5)$ & $0.5,1.0)$ & 100 & -0.22 & 59.54 & -1701 & 1702 \\
& & 200 & $-4.1 \mathrm{e}-2$ & -22.05 & -1127 & 1128 \\
& $(0,0.25$, & 500 & $-1.9 \mathrm{e}-2$ & 4.48 & -167 & 167 \\
\cline { 2 - 7 } & $0.375,0.5$, & 100 & $-7.8 \mathrm{e}-2$ & 0.11 & -41.58 & 41.58 \\
& $0.75,1.0)$ & 200 & $-3.7 \mathrm{e}-2$ & -2.30 & -28.33 & 28.42 \\
& & 500.29 & 15.17 & -85.45 & 86.79 \\
& $0.375,0.5$, & 100 & -0.15 & -7.31 & -40.67 & 41.32 \\
& $0.75,1.0)$ & 200 & $-7.1 \mathrm{e}-2$ & 9.42 & -30.60 & 32.02 \\
& & 500 & $-2.8 \mathrm{e}-2$ & 6.19 & -19.23 & 20.20 \\
\hline \hline
\end{tabular}

initial slope.

Table A.8: Properties of Covariance of MLE for $\kappa$ and $\eta$ 


\begin{tabular}{|c|c|c|c|c|c|c|}
\hline $\begin{array}{c}\text { Dose-Respn } \\
\text { Shape } \\
\left(\alpha, \kappa / d_{t}, \eta\right)\end{array}$ & $\begin{array}{c}\text { Dose Groups } \\
\left(d_{0}, \cdots, d_{t}\right) \\
0 \rightarrow 8 \mathrm{mg} / \mathrm{kg}\end{array}$ & $\begin{array}{c}\text { Size } / \\
\text { Dose Group } \\
n_{i}\end{array}$ & $\begin{array}{l}\text { Cov. } \\
\text { Est. } \\
(\%)\end{array}$ & $\begin{array}{l}\text { Rel. } \\
\text { Bias } \\
(\%)\end{array}$ & $\begin{array}{c}\text { Rel. } \\
\text { Stdev } \\
(\%) \\
\end{array}$ & $\begin{array}{l}\text { Rel. } \\
\text { MSE } \\
(\%)\end{array}$ \\
\hline \multirow{8}{*}{$\begin{array}{c}\text { S-shaped } \\
(0.01,0.500,3)\end{array}$} & $(0,0.25$, & 50 & $2.1 \mathrm{e}-3$ & 323 & $\overline{303}$ & $\overline{423}$ \\
\hline & $0.5,1.0)$ & 100 & $9.2 \mathrm{e}-4$ & 1603 & 620 & 1719 \\
\hline & & 200 & $4.2 \mathrm{e}-4$ & 1117 & 339 & 1167 \\
\hline & & 500 & $1.7 \mathrm{e}-4$ & 2821 & 430 & 2854 \\
\hline & $(0,0.25$ & 50 & $1.8 \mathrm{e}-3$ & 527 & 377 & 648 \\
\hline & $0.375,0.5$ & 100 & $8.2 \mathrm{e}-4$ & 924 & 367 & 995 \\
\hline & $0.75,1.0)$ & 200 & $3.7 \mathrm{e}-4$ & 757 & 211 & 786 \\
\hline & & 500 & $1.5 \mathrm{e}-5$ & 1676 & 242 & 1694 \\
\hline \multirow{8}{*}{$\begin{array}{c}\text { Convex } \\
(0.01,0.625,1)\end{array}$} & $(0,0.25$ & 50 & $5.1 \mathrm{e}-4$ & -153 & -890 & 902 \\
\hline & $0.5,1.0)$ & 100 & $2.5 \mathrm{e}-3$ & 1203 & 549 & 1323 \\
\hline & & 200 & $1.1 \mathrm{e}-3$ & 2477 & 648 & 2560 \\
\hline & & 500 & $4.3 \mathrm{e}-4$ & 3187 & 427 & 3216 \\
\hline & $(0,0.25$ & 50 & $2.7 \mathrm{e}-3$ & 116 & 12137 & 12138 \\
\hline & $0.375,0.5$ & 100 & $2.1 \mathrm{e}-4$ & 927 & 209 & 950 \\
\hline & $0.75,1.0)$ & 200 & $1.1 \mathrm{e}-3$ & 931 & 252 & 964 \\
\hline & & 500 & $4.2 \mathrm{e}-4$ & 6618 & 790 & 6665 \\
\hline \multirow{8}{*}{$\begin{array}{c}\text { Concave } \\
(>0) \\
(0.01,0.875,2)\end{array}$} & $(0,0.25$ & 50 & $2.8 \mathrm{e}-3$ & 476 & 378 & 608 \\
\hline & $0.5,1.0)$ & 100 & $1.3 \mathrm{e}-3$ & 673 & 233 & 712 \\
\hline & & 200 & $6.4 \mathrm{e}-4$ & 4228 & 828 & 4309 \\
\hline & & 500 & $2.6 \mathrm{e}-4$ & 4157 & 417 & 4178 \\
\hline & $(0,0.25$ & 50 & $2.4 \mathrm{e}-3$ & 408 & 291 & 501 \\
\hline & $0.375,0.5$ & 100 & $1.1 \mathrm{e}-3$ & 643 & 201 & 674 \\
\hline & $0.75,1.0)$ & 200 & $5.4 \mathrm{e}-4$ & 488 & 106 & 500 \\
\hline & & 500 & $2.2 \mathrm{e}-4$ & 2590 & 267 & 2604 \\
\hline \multirow{8}{*}{$\begin{array}{c}\text { Concave } \\
(=0) \\
(0.01,1.000,5)\end{array}$} & $(0,0.25$ & 50 & $1.6 \mathrm{e}-4$ & 54.48 & 1866 & 1866 \\
\hline & $0.5,1.0)$ & 100 & $1.7 \mathrm{e}-4$ & 63.62 & 387 & 392 \\
\hline & & 200 & $1.0 \mathrm{e}-4$ & 204 & 355 & 410 \\
\hline & & 500 & $4.3 \mathrm{e}-6$ & 158 & 155 & 221 \\
\hline & $(0,0.25$ & 50 & $-1.1 \mathrm{e}-4$ & -169 & 458 & 489 \\
\hline & $0.375,0.5$ & 100 & $-5.8 \mathrm{e}-5$ & -201 & 408 & 455 \\
\hline & $0.75,1.0)$ & 200 & $-2.5 \mathrm{e}-6$ & 11.10 & -279 & 279 \\
\hline & & 500 & $-9.6 e-6$ & -312 & 340 & 461 \\
\hline
\end{tabular}

initial slope.

Table A.9: Properties of Covariance of MLE for $\kappa$ and $\alpha$ 


\begin{tabular}{|c|c|c|c|c|c|c|}
\hline $\begin{array}{c}\text { Dose-Respn } \\
\text { Shape } \\
\left(\alpha, \kappa / d_{t}, \eta\right)\end{array}$ & $\begin{array}{c}\text { Dose Groups } \\
\left(d_{0}, \cdots, d_{t}\right) \\
0 \rightarrow 8 \mathrm{mg} / \mathrm{kg}\end{array}$ & $\begin{array}{c}\text { Size } / \\
\text { Dose Group } \\
n_{i}\end{array}$ & $\begin{array}{l}\text { Cov. } \\
\text { Est. } \\
(\%)\end{array}$ & $\begin{array}{l}\text { Rel. } \\
\text { Bias } \\
(\%)\end{array}$ & $\begin{array}{l}\text { Rel. } \\
\text { Stdev } \\
(\%) \\
\end{array}$ & $\begin{array}{l}\text { Rel. } \\
\text { MSE } \\
(\%)\end{array}$ \\
\hline \multirow{8}{*}{$\begin{array}{c}\text { S-shaped } \\
(0.01,0.500,3)\end{array}$} & \multirow{4}{*}{$\begin{array}{l}(0,0.25 \\
0.5,1.0)\end{array}$} & 50 &  & $\bar{~} 53.99$ & 226 & 232 \\
\hline & & 100 & $1.3 \mathrm{e}-3$ & 654 & 506 & 827 \\
\hline & & 200 & $5.7 \mathrm{e}-4$ & 996 & 571 & 1148 \\
\hline & & 500 & $2.2 \mathrm{e}-4$ & 3803 & 880 & 3904 \\
\hline & \multirow{4}{*}{$\begin{array}{c}(0,0.25, \\
0.375,0.5 \\
0.75,1.0)\end{array}$} & 50 & $2.6 \mathrm{e}-3$ & 315 & 436 & 538 \\
\hline & & 100 & $1.1 \mathrm{e}-3$ & 428 & 324 & 537 \\
\hline & & 200 & $4.6 \mathrm{e}-4$ & 353 & 167 & 391 \\
\hline & & 500 & $1.8 \mathrm{e}-4$ & 812 & 192 & 834 \\
\hline \multirow{8}{*}{$\begin{array}{c}\text { Convex } \\
(0.01,0.625,1)\end{array}$} & \multirow{4}{*}{$\begin{array}{l}(0,0.25 \\
0.5,1.0)\end{array}$} & 50 & $7.8 \mathrm{e}-4$ & 212 & 696 & 728 \\
\hline & & 100 & $3.1 \mathrm{e}-4$ & -1133 & -417 & 1207 \\
\hline & & 200 & $1.4 \mathrm{e}-4$ & 406 & 140 & 429 \\
\hline & & 500 & $5.5 \mathrm{e}-5$ & 16150 & 2510 & 16344 \\
\hline & \multirow{4}{*}{$\begin{array}{c}(0,0.25, \\
0.375,0.5 \\
0.75,1.0)\end{array}$} & 50 & $6.5 \mathrm{e}-4$ & 159 & 558 & 580 \\
\hline & & 100 & $3.0 \mathrm{e}-4$ & 310 & 151 & 345 \\
\hline & & 200 & $1.4 \mathrm{e}-4$ & -889 & -194 & 910 \\
\hline & & 500 & $5.4 \mathrm{e}-5$ & 1284 & 188 & 1298 \\
\hline \multirow{8}{*}{$\begin{array}{c}\text { Concave } \\
(>0) \\
(0.01,0.875,2)\end{array}$} & \multirow{4}{*}{$\begin{array}{l}(0,0.25 \\
0.5,1.0)\end{array}$} & 50 & $4.8 \mathrm{e}-3$ & 182 & 439 & 476 \\
\hline & & 100 & $1.6 \mathrm{e}-3$ & 865 & 688 & 1105 \\
\hline & & 200 & $7.1 \mathrm{e}-4$ & 528 & 332 & 624 \\
\hline & & 500 & $2.8 \mathrm{e}-4$ & 1519 & 402 & 1572 \\
\hline & \multirow{4}{*}{$\begin{array}{c}(0,0.25, \\
0.375,0.5 \\
0.75,1.0)\end{array}$} & 50 & 0.72 & 13.70 & 182 & 182 \\
\hline & & 100 & $1.5 \mathrm{e}-3$ & 732 & 647 & 977 \\
\hline & & 200 & $6.6 \mathrm{e}-4$ & 738 & 341 & 814 \\
\hline & & 500 & $2.6 \mathrm{e}-4$ & 1389 & 327 & 1428 \\
\hline \multirow{8}{*}{$\begin{array}{c}\text { Concave } \\
(=0) \\
(0.01,1.000,5)\end{array}$} & \multirow{4}{*}{$\begin{array}{l}(0,0.25 \\
0.5,1.0)\end{array}$} & 50 & $2.2 \mathrm{e}-2$ & 1103 & 4035 & 4184 \\
\hline & & 100 & $8.2 \mathrm{e}-3$ & 396 & 1108 & 1177 \\
\hline & & 200 & $4.2 \mathrm{e}-3$ & 145 & 511 & 531 \\
\hline & & 500 & $1.2 \mathrm{e}-3$ & 287 & 363 & 462 \\
\hline & \multirow{4}{*}{$\begin{array}{c}(0,0.25, \\
0.375,0.5 \\
0.75,1.0)\end{array}$} & 50 & $5.1 \mathrm{e}-3$ & 61.04 & 163 & 174 \\
\hline & & 100 & $2.5 \mathrm{e}-3$ & 152 & 170 & 228 \\
\hline & & 200 & $1.2 \mathrm{e}-3$ & 77.42 & 88.35 & 117 \\
\hline & & 500 & $5.0 \mathrm{e}-4$ & 228 & 99.78 & 249 \\
\hline
\end{tabular}

initial slope.

Table A.10: Properties of Covariance of MLE for $\alpha$ and $\eta$ 


\section{A.3.4 Properties of Benchmark Dose Estimates based on Extra Risk}

Tables summarize relative bias, that of std, that of MSE and confidence coverage for BMD estimates (based on extra risk) of BMR of $01 \%, 05 \%$ and $10 \%$ (Chapter 2).

\begin{tabular}{|c|c|c|c|c|c|c|c|}
\hline $\begin{array}{c}\text { Dose-Respn } \\
\text { Shape } \\
\left(\alpha, \kappa / d_{t}, \eta\right)\end{array}$ & $\begin{array}{c}\text { Dose Groups } \\
\left(d_{0}, \cdots, d_{t}\right) \\
0 \rightarrow 8 \mathrm{mg} / \mathrm{kg}\end{array}$ & $\begin{array}{c}\text { Size/ } \\
\text { Dose Group } \\
n_{i}\end{array}$ & $\begin{array}{c}\text { True } \\
\text { BMD }\end{array}$ & $\begin{array}{c}\text { Rel. } \\
\text { Bias } \\
(\%)\end{array}$ & $\begin{array}{c}\text { Rel. } \\
\text { Stdev } \\
(\%)\end{array}$ & $\begin{array}{c}\text { Rel. } \\
\text { MSE } \\
(\%)\end{array}$ & $\begin{array}{c}\text { Conf. } \\
\text { Cov. } \\
(\%)\end{array}$ \\
\hline \hline S-shaped & $(0,0.25$, & 50 & 0.86 & 11.20 & 30.40 & 32.40 & 0.919 \\
$(0.01,0.500,3)$ & $0.5,1.0)$ & 100 & 0.86 & 5.92 & 20.08 & 20.93 & 0.926 \\
& & 200 & 0.86 & 4.79 & 13.93 & 14.73 & 0.896 \\
& & 500 & 0.86 & 4.14 & 8.76 & 9.69 & 0.878 \\
\cline { 2 - 8 } & $(0,0.25$, & 50 & 0.86 & 7.11 & 23.44 & 24.49 & 0.907 \\
& $0.375,0.5$, & 100 & 0.86 & 5.28 & 16.14 & 16.99 & 0.902 \\
& $0.75,1.0)$ & 200 & 0.86 & 3.55 & 11.19 & 11.74 & 0.890 \\
& & 500 & 0.86 & 3.39 & 7.07 & 7.83 & 0.876 \\
\hline \hline Convex & $(0,0.25$, & 50 & 0.05 & 73.46 & 196 & 209 & 0.816 \\
$(0.01,0.625,1)$ & $0.5,1.0)$ & 100 & 0.05 & 41.88 & 124 & 131 & 0.857 \\
& & 200 & 0.05 & 25.60 & 81.37 & 85.30 & 0.881 \\
& & 500 & 0.05 & 12.94 & 48.50 & 50.20 & 0.912 \\
\cline { 2 - 8 } & $(0,0.25$, & 50 & 0.05 & 57.02 & 164 & 173 & 0.839 \\
& $0.375,0.5$, & 100 & 0.05 & 30.96 & 105 & 109 & 0.870 \\
& $0.75,1.0)$ & 200 & 0.05 & 23.89 & 71.31 & 75.21 & 0.895 \\
& & 500 & 0.05 & 14.38 & 43.18 & 45.51 & 0.901 \\
\hline \hline Concave & $0,0.25$, & 50 & 0.70 & 24.18 & 62.67 & 67.18 & 0.922 \\
$(>0)$ & $0.5,1.0)$ & 100 & 0.70 & 13.95 & 37.74 & 40.23 & 0.929 \\
$(0.01,0.875,2)$ & & 200 & 0.70 & 10.58 & 25.65 & 27.75 & 0.908 \\
& & 500 & 0.70 & 9.64 & 16.05 & 18.73 & 0.887 \\
\cline { 2 - 7 } & $(0,0.25$, & 50 & 0.70 & 18.25 & 48.49 & 51.81 & 0.905 \\
& $0.375,0.5$, & 100 & 0.70 & 12.06 & 31.41 & 33.64 & 0.923 \\
& $0.75,1.0)$ & 200 & 0.70 & 8.92 & 21.36 & 23.15 & 0.921 \\
& & 500 & 0.70 & 8.54 & 13.46 & 15.94 & 0.885 \\
\hline \hline & $0.375,0.5$, & 100 & 3.19 & 5.41 & 13.79 & 14.81 & 0.885 \\
& & 200 & 3.19 & 3.79 & 9.77 & 10.48 & 0.892 \\
& & 500 & 3.19 & 3.90 & 6.19 & 7.32 & 0.873 \\
\hline \hline
\end{tabular}

initial slope.

Table A.11: Properties of Estimate of $B M D 01$ based on Extra Risk 


\begin{tabular}{|c|c|c|c|c|c|c|c|}
\hline $\begin{array}{c}\text { Dose-Respn } \\
\text { Shape } \\
\left(\alpha, \kappa / d_{t}, \eta\right)\end{array}$ & $\begin{array}{c}\text { Dose Groups } \\
\left(d_{0}, \cdots, d_{t}\right) \\
0 \rightarrow 8 \mathrm{mg} / \mathrm{kg}\end{array}$ & $\begin{array}{c}\text { Size/ } \\
\text { Dose Group } \\
n_{i}\end{array}$ & $\begin{array}{l}\text { True } \\
\text { BMD }\end{array}$ & $\begin{array}{l}\text { Rel. } \\
\text { Bias } \\
(\%)\end{array}$ & $\begin{array}{c}\text { Rel. } \\
\text { Stdev } \\
(\%)\end{array}$ & $\begin{array}{l}\text { Rel. } \\
\text { MSE } \\
(\%)\end{array}$ & $\begin{array}{l}\text { Conf. } \\
\text { Cov. } \\
(\%)\end{array}$ \\
\hline \multirow{8}{*}{$\begin{array}{c}\text { S-shaped } \\
(0.01,0.500,3)\end{array}$} & $(0,0.25$, & $\overline{50}$ & 1.50 & $\overline{6.91}$ & 20.12 & 21.27 & 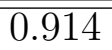 \\
\hline & $0.5,1.0)$ & 100 & 1.50 & 3.84 & 13.54 & 14.08 & 0.922 \\
\hline & & 200 & 1.50 & 3.31 & 9.43 & 9.99 & 0.891 \\
\hline & & 500 & 1.50 & 3.00 & 5.95 & 6.66 & 0.868 \\
\hline & $(0,0.25$ & 50 & 1.50 & 4.50 & 15.74 & 16.37 & 0.905 \\
\hline & $0.375,0.5$ & 100 & 1.50 & 3.62 & 10.89 & 11.48 & 0.899 \\
\hline & $0.75,1.0)$ & 200 & 1.50 & 2.49 & 7.57 & 7.97 & 0.885 \\
\hline & & 500 & 1.50 & 2.46 & 4.78 & 5.76 & 0.870 \\
\hline \multirow{8}{*}{$\begin{array}{c}\text { Convex } \\
(0.01,0.625,1)\end{array}$} & $(0,0.25$ & 50 & 0.26 & 26.72 & 93.95 & 97.68 & 0.858 \\
\hline & $0.5,1.0)$ & 100 & 0.26 & 15.93 & 65.20 & 67.12 & 0.860 \\
\hline & & 200 & 0.26 & 11.25 & 46.03 & 47.38 & 0.887 \\
\hline & & 500 & 0.26 & 6.84 & 29.12 & 29.91 & 0.897 \\
\hline & $(0,0.25$ & 50 & 0.26 & 20.56 & 81.62 & 84.17 & 0.865 \\
\hline & $0.375,0.5$ & 100 & 0.26 & 12.26 & 57.56 & 58.86 & 0.886 \\
\hline & $0.75,1.0)$ & 200 & 0.26 & 12.14 & 43.57 & 45.23 & 0.917 \\
\hline & & 500 & 0.26 & 8.02 & 25.88 & 27.10 & 0.895 \\
\hline \multirow{8}{*}{$\begin{array}{c}\text { Concave } \\
(>0) \\
(0.01,0.875,2)\end{array}$} & $(0,0.25$ & 50 & 1.61 & 12.90 & 35.39 & 37.67 & $\bar{~} 0.929$ \\
\hline & $0.5,1.0)$ & 100 & 1.61 & 8.10 & 22.68 & 24.09 & 0.931 \\
\hline & & 200 & 1.61 & 6.47 & 15.57 & 16.86 & 0.905 \\
\hline & & 500 & 1.61 & 6.24 & 9.78 & 11.61 & 0.862 \\
\hline & $(0,0.25$ & 50 & 1.61 & 9.62 & 28.06 & 29.66 & 0.909 \\
\hline & $0.375,0.5$ & 100 & 1.61 & 7.22 & 18.71 & 20.06 & 0.920 \\
\hline & $0.75,1.0)$ & 200 & 1.61 & 5.53 & 12.85 & 13.99 & 0.917 \\
\hline & & 500 & 1.61 & 5.56 & 8.10 & 9.82 & 0.856 \\
\hline \multirow{8}{*}{$\begin{array}{c}\text { Concave } \\
(=0) \\
(0.01,1.000,5)\end{array}$} & $(0,0.25$ & 50 & 4.44 & 13.54 & 45.56 & 47.53 & 0.786 \\
\hline & $0.5,1.0)$ & 100 & 4.44 & 9.18 & 24.16 & 25.85 & 0.866 \\
\hline & & 200 & 4.44 & 6.43 & 15.10 & 16.41 & 0.955 \\
\hline & & 500 & 4.44 & 4.26 & 6.97 & 8.17 & 0.951 \\
\hline & $(0,0.25$ & 50 & 4.44 & 4.06 & 11.74 & 12.43 & 0.882 \\
\hline & $0.375,0.5$ & 100 & 4.44 & 3.24 & 8.35 & 8.96 & 0.882 \\
\hline & $0.75,1.0)$ & 200 & 4.44 & 2.31 & 5.94 & 6.37 & 0.900 \\
\hline & & 500 & 4.44 & 2.41 & 3.76 & 4.47 & 0.869 \\
\hline
\end{tabular}

initial slope.

Table A.12: Properties of Estimate of $B M D 05$ based on Extra Risk 


\begin{tabular}{|c|c|c|c|c|c|c|c|}
\hline $\begin{array}{c}\text { Dose-Respn } \\
\text { Shape } \\
\left(\alpha, \kappa / d_{t}, \eta\right)\end{array}$ & $\begin{array}{c}\text { Dose Groups } \\
\left(d_{0}, \cdots, d_{t}\right) \\
0 \rightarrow 8 \mathrm{mg} / \mathrm{kg}\end{array}$ & $\begin{array}{c}\text { Size/ } \\
\text { Dose Group } \\
n_{i}\end{array}$ & $\begin{array}{l}\text { True } \\
\text { BMD }\end{array}$ & $\begin{array}{l}\text { Rel. } \\
\text { Bias } \\
(\%)\end{array}$ & $\begin{array}{c}\text { Rel. } \\
\text { Stdev } \\
(\%)\end{array}$ & $\begin{array}{l}\text { Rel. } \\
\text { MSE } \\
(\%)\end{array}$ & $\begin{array}{l}\text { Conf. } \\
\text { Cov. } \\
(\%)\end{array}$ \\
\hline \multirow{8}{*}{$\begin{array}{c}\text { S-shaped } \\
(0.01,0.500,3)\end{array}$} & $(0,0.25$, & $\overline{50}$ & 1.92 & 5.34 & $\bar{~} 15.99$ & 16.85 &  \\
\hline & $0.5,1.0)$ & 100 & 1.92 & 3.03 & 10.82 & 11.24 & 0.920 \\
\hline & & 200 & 1.92 & 2.71 & 7.54 & 8.02 & 0.896 \\
\hline & & 500 & 1.92 & 2.51 & 4.76 & 5.38 & 0.862 \\
\hline & $(0,0.25$ & 50 & 1.92 & 3.51 & 12.52 & 13.00 & 0.904 \\
\hline & $0.375,0.5$ & 100 & 1.92 & 2.96 & 8.67 & 9.16 & 0.894 \\
\hline & $0.75,1.0)$ & 200 & 1.92 & 2.06 & 6.03 & 6.37 & 0.884 \\
\hline & & 500 & 1.92 & 2.06 & 3.80 & 4.32 & 0.859 \\
\hline \multirow{8}{*}{$\begin{array}{c}\text { Convex } \\
(0.01,0.625,1)\end{array}$} & $(0,0.25$ & 50 & 0.56 & 14.25 & 66.25 & 67.76 & 0.868 \\
\hline & $0.5,1.0)$ & 100 & 0.56 & 8.79 & 47.02 & 47.84 & 0.866 \\
\hline & & 200 & 0.56 & 7.06 & 33.51 & 34.25 & 0.889 \\
\hline & & 500 & 0.56 & 4.99 & 21.41 & 21.98 & 0.891 \\
\hline & $(0,0.25$ & 50 & 0.56 & 10.79 & 58.16 & 59.16 & 0.869 \\
\hline & $0.375,0.5$ & 100 & 0.56 & 7.08 & 41.78 & 42.37 & 0.890 \\
\hline & $0.75,1.0)$ & 200 & 0.56 & 7.26 & 29.67 & 30.55 & 0.891 \\
\hline & & 500 & 0.56 & 5.91 & 18.93 & 19.83 & 0.890 \\
\hline \multirow{8}{*}{$\begin{array}{c}\text { Concave } \\
(>0) \\
(0.01,0.875,2)\end{array}$} & $(0,0.25$ & 50 & 2.33 & 9.26 & 25.60 & 27.22 & 0.927 \\
\hline & $0.5,1.0)$ & 100 & 2.33 & 6.07 & 16.70 & 17.77 & 0.924 \\
\hline & & 200 & 2.33 & 4.91 & 11.47 & 12.48 & 0.908 \\
\hline & & 500 & 2.33 & 4.84 & 7.21 & 8.69 & 0.859 \\
\hline & $(0,0.25$ & 50 & 2.33 & 6.82 & 20.25 & 21.37 & 0.907 \\
\hline & $0.375,0.5$ & 100 & 2.33 & 5.46 & 13.57 & 14.63 & 0.912 \\
\hline & $0.75,1.0)$ & 200 & 2.33 & 4.21 & 9.33 & 10.24 & 0.900 \\
\hline & & 500 & 2.33 & 4.30 & 5.88 & 7.29 & 0.846 \\
\hline \multirow{8}{*}{$\begin{array}{c}\text { Concave } \\
(=0) \\
(0.01,1.000,5)\end{array}$} & $(0,0.25$ & 50 & 5.16 & 9.66 & 31.43 & 32.88 & 0.786 \\
\hline & $0.5,1.0)$ & 100 & 5.16 & 6.60 & 17.01 & 18.24 & 0.864 \\
\hline & & 200 & 5.16 & 4.67 & 10.81 & 11.78 & 0.956 \\
\hline & & 500 & 5.16 & 3.18 & 5.16 & 6.06 & 0.939 \\
\hline & $(0,0.25$ & 50 & 5.16 & 2.89 & 8.55 & 9.03 & 0.877 \\
\hline & $0.375,0.5$ & 100 & 5.16 & 2.37 & 6.09 & 6.54 & 0.884 \\
\hline & $0.75,1.0)$ & 200 & 5.16 & 1.68 & 4.34 & 4.65 & 0.895 \\
\hline & & 500 & 5.16 & 1.75 & 2.75 & 3.26 & 0.862 \\
\hline
\end{tabular}

initial slope.

Table A.13: Properties of Estimate of $B M D 10$ based on Extra Risk 


\section{A.3.5 Properties of Benchmark Dose Estimates based on Additional}

Risk

Tables summarize relative bias, that of std, that of MSE and confidence coverage for BMD estimates (based on additional risk) of BMR of $01 \%, 05 \%$ and $10 \%$.

\begin{tabular}{|c|c|c|c|c|c|c|c|}
\hline $\begin{array}{c}\text { Dose-Respn } \\
\text { Shape } \\
\left(\alpha, \kappa / d_{t}, \eta\right)\end{array}$ & $\begin{array}{c}\text { Dose Groups } \\
\left(d_{0}, \cdots, d_{t}\right) \\
0 \rightarrow 8 \mathrm{mg} / \mathrm{kg}\end{array}$ & $\begin{array}{c}\text { Size/ } \\
\text { Dose Group } \\
n_{i}\end{array}$ & $\begin{array}{c}\text { True } \\
\text { BMD }\end{array}$ & $\begin{array}{c}\text { Rel. } \\
\text { Bias } \\
(\%)\end{array}$ & $\begin{array}{c}\text { Rel. } \\
\text { Stdev } \\
(\%)\end{array}$ & $\begin{array}{c}\text { Rel. } \\
\text { MSE } \\
(\%)\end{array}$ & $\begin{array}{c}\text { Conf. } \\
\text { Cov. } \\
(\%)\end{array}$ \\
\hline \hline S-shaped & $(0,0.25$, & 50 & 0.87 & 11.64 & 29.25 & 31.48 & 0.900 \\
$(0.01,0.500,3)$ & $0.5,1.0)$ & 100 & 0.87 & 6.29 & 19.47 & 20.46 & 0.907 \\
& & 200 & 0.87 & 5.11 & 13.56 & 14.48 & 0.891 \\
& & 500 & 0.87 & 4.47 & 8.53 & 9.63 & 0.856 \\
\cline { 2 - 8 } & $(0,0.25$, & 50 & 0.87 & 7.53 & 22.52 & 23.74 & 0.892 \\
& $0.375,0.5$, & 100 & 0.87 & 5.66 & 15.60 & 16.60 & 0.887 \\
& $0.75,1.0)$ & 200 & 0.87 & 3.87 & 10.84 & 11.51 & 0.873 \\
& & 500 & 0.87 & 3.72 & 6.85 & 7.79 & 0.856 \\
\hline \hline Convex & $(0,0.25$, & 50 & 0.05 & 74.83 & 206 & 219 & 0.825 \\
$(0.01,0.625,1)$ & $0.5,1.0)$ & 100 & 0.05 & 43.08 & 130 & 137 & 0.863 \\
& & 200 & 0.05 & 26.61 & 85.21 & 89.27 & 0.891 \\
& & 500 & 0.05 & 13.98 & 50.90 & 52.79 & 0.921 \\
\cline { 2 - 8 } & $(0,0.25$, & 50 & 0.05 & 58.32 & 171 & 181 & 0.845 \\
& $0.375,0.5$, & 100 & 0.05 & 32.21 & 109 & 114 & 0.880 \\
& $0.75,1.0)$ & 200 & 0.05 & 24.91 & 74.42 & 78.48 & 0.905 \\
& & 500 & 0.05 & 15.44 & 45.12 & 47.69 & 0.913 \\
\hline \hline Concave & $(0,0.25$, & 50 & 0.71 & 24.85 & 67.66 & 72.08 & 0.939 \\
$(>0)$ & $0.5,1.0)$ & 100 & 0.71 & 14.50 & 41.83 & 44.27 & 0.950 \\
$(0.01,0.875,2)$ & & 200 & 0.71 & 11.06 & 28.57 & 30.64 & 0.945 \\
& & 500 & 0.71 & 10.13 & 17.92 & 20.59 & 0.917 \\
\cline { 2 - 7 } & $(0,0.25$, & 50 & 0.71 & 18.89 & 53.85 & 57.06 & 0.931 \\
& $0.375,0.5$, & 100 & 0.71 & 12.61 & 35.50 & 37.67 & 0.958 \\
& $0.75,1.0)$ & 200 & 0.71 & 9.40 & 24.24 & 26.00 & 0.952 \\
& & 500 & 0.71 & 9.03 & 15.30 & 17.76 & 0.920 \\
\hline \hline & $0.375,0.5$, & 100 & 3.19 & 5.48 & 15.95 & 16.87 & 0.915 \\
& $0.75,1.0)$ & 200 & 3.19 & 3.86 & 11.28 & 11.92 & 0.931 \\
& & 500 & 3.19 & 3.97 & 7.14 & 8.17 & 0.914 \\
\hline \hline Concave & $(0,0.25$, & 50 & 3.19 & 23.91 & 86.74 & 89.97 & 0.791 \\
$(=0)$ & $0.5,1.0)$ & 100 & 3.19 & 15.98 & 44.14 & 46.94 & 0.871 \\
$(0.01,1.000,5)$ & & 200 & 3.19 & 10.84 & 26.52 & 28.65 & 0.958 \\
& & & & & & & \\
\cline { 2 - 7 } & $(0,0.25$, & 500 & 3.19 & 6.87 & 11.62 & 13.50 & 0.976 \\
\hline
\end{tabular}

initial slope.

Table A.14: Properties of Estimate of BMD01 based on Additional Risk 


\begin{tabular}{|c|c|c|c|c|c|c|c|}
\hline $\begin{array}{c}\text { Dose-Respn } \\
\text { Shape } \\
\left(\alpha, \kappa / d_{t}, \eta\right)\end{array}$ & $\begin{array}{c}\text { Dose Groups } \\
\left(d_{0}, \cdots, d_{t}\right) \\
0 \rightarrow 8 \mathrm{mg} / \mathrm{kg}\end{array}$ & $\begin{array}{c}\text { Size/ } \\
\text { Dose Group } \\
n_{i}\end{array}$ & $\begin{array}{l}\text { True } \\
\text { BMD }\end{array}$ & $\begin{array}{l}\text { Rel. } \\
\text { Bias } \\
(\%)\end{array}$ & $\begin{array}{c}\text { Rel. } \\
\text { Stdev } \\
(\%)\end{array}$ & $\begin{array}{l}\text { Rel. } \\
\text { MSE } \\
(\%)\end{array}$ & $\begin{array}{l}\text { Conf. } \\
\text { Cov. } \\
(\%)\end{array}$ \\
\hline \multirow{8}{*}{$\begin{array}{c}\text { S-shaped } \\
(0.01,0.500,3)\end{array}$} & $(0,0.25$, & $\overline{50}$ & 1.50 & 7.35 & $\bar{~} 19.16$ & 20.53 & $\overline{00.894}$ \\
\hline & $0.5,1.0)$ & 100 & 1.50 & 4.22 & 13.01 & 13.68 & 0.894 \\
\hline & & 200 & 1.50 & 3.63 & 9.09 & 9.79 & 0.877 \\
\hline & & 500 & 1.50 & 3.34 & 5.74 & 6.64 & 0.840 \\
\hline & $(0,0.25$ & 50 & 1.50 & 4.93 & 14.95 & 15.74 & 0.881 \\
\hline & $0.375,0.5$ & 100 & 1.50 & 4.01 & 10.41 & 11.16 & 0.868 \\
\hline & $0.75,1.0)$ & 200 & 1.50 & 2.82 & 7.26 & 7.79 & 0.865 \\
\hline & & 500 & 1.50 & 2.80 & 4.58 & 5.37 & 0.843 \\
\hline \multirow{8}{*}{$\begin{array}{c}\text { Convex } \\
(0.01,0.625,1)\end{array}$} & $(0,0.25$ & 50 & 0.27 & 27.95 & 103 & 106 & 0.873 \\
\hline & $0.5,1.0)$ & 100 & 0.27 & 17.07 & 70.34 & 70.38 & 0.882 \\
\hline & & 200 & 0.27 & 12.25 & 49.44 & 50.94 & 0.905 \\
\hline & & 500 & 0.27 & 7.89 & 31.30 & 32.28 & 0.915 \\
\hline & $(0,0.25$ & 50 & 0.27 & 21.77 & 88.07 & 90.72 & 0.881 \\
\hline & $0.375,0.5$ & 100 & 0.27 & 13.47 & 61.76 & 63.21 & 0.902 \\
\hline & $0.75,1.0)$ & 200 & 0.27 & 12.14 & 43.57 & 45.23 & 0.917 \\
\hline & & 500 & 0.27 & 9.08 & 27.64 & 29.10 & 0.908 \\
\hline \multirow{8}{*}{$\begin{array}{c}\text { Concave } \\
(>0) \\
(0.01,0.875,2)\end{array}$} & $(0,0.25$ & 50 & 1.61 & 13.56 & 41.11 & 433.29 & 0.948 \\
\hline & $0.5,1.0)$ & 100 & 1.61 & 8.66 & 26.85 & 28.22 & 0.958 \\
\hline & & 200 & 1.61 & 6.95 & 18.45 & 19.71 & 0.945 \\
\hline & & 500 & 1.61 & 6.74 & 11.58 & 13.40 & 0.912 \\
\hline & $(0,0.25$ & 50 & 1.61 & 10.27 & 33.69 & 35.22 & 0.942 \\
\hline & $0.375,0.5$ & 100 & 1.61 & 7.78 & 22.80 & 24.09 & 0.955 \\
\hline & $0.75,1.0)$ & 200 & 1.61 & 6.02 & 15.69 & 16.81 & 0.956 \\
\hline & & 500 & 1.61 & 6.06 & 9.89 & 11.60 & 0.917 \\
\hline \multirow{8}{*}{$\begin{array}{c}\text { Concave } \\
(=0) \\
(0.01,1.000,5)\end{array}$} & $(0,0.25$ & 50 & 4.45 & 13.58 & 47.63 & 49.52 & 0.795 \\
\hline & $0.5,1.0)$ & 100 & 4.45 & 9.26 & 24.83 & 26.50 & 0.874 \\
\hline & & 200 & 4.45 & 6.50 & 15.38 & 16.70 & 0.963 \\
\hline & & 500 & 4.45 & 4.35 & 7.26 & 8.47 & 0.957 \\
\hline & $(0,0.25$ & 50 & 4.45 & 4.15 & 14.91 & 15.48 & 0.919 \\
\hline & $0.375,0.5$ & 100 & 4.45 & 3.32 & 10.53 & 11.04 & 0.934 \\
\hline & $0.75,1.0)$ & 200 & 4.45 & 2.38 & 7.43 & 7.80 & 0.942 \\
\hline & & 500 & 4.45 & 2.48 & 4.69 & 5.31 & 0.933 \\
\hline
\end{tabular}

initial slope.

Table A.15: Properties of Estimate of $B M D 05$ based on Additional Risk 


\begin{tabular}{|c|c|c|c|c|c|c|c|}
\hline $\begin{array}{c}\text { Dose-Respn } \\
\text { Shape } \\
\left(\alpha, \kappa / d_{t}, \eta\right)\end{array}$ & $\begin{array}{c}\text { Dose Groups } \\
\left(d_{0}, \cdots, d_{t}\right) \\
0 \rightarrow 8 \mathrm{mg} / \mathrm{kg}\end{array}$ & $\begin{array}{c}\text { Size/ } \\
\text { Dose Group } \\
n_{i}\end{array}$ & $\begin{array}{l}\text { True } \\
\text { BMD }\end{array}$ & $\begin{array}{l}\text { Rel. } \\
\text { Bias } \\
(\%)\end{array}$ & $\begin{array}{c}\text { Rel. } \\
\text { Stdev } \\
(\%)\end{array}$ & $\begin{array}{l}\text { Rel. } \\
\text { MSE } \\
(\%)\end{array}$ & $\begin{array}{l}\text { Conf. } \\
\text { Cov. } \\
(\%)\end{array}$ \\
\hline \multirow{8}{*}{$\begin{array}{c}\text { S-shaped } \\
(0.01,0.500,3)\end{array}$} & $(0,0.25$, & $\overline{50}$ & 1.93 & 5.80 & $\bar{~} 15.17$ & 16.24 & 0.885 \\
\hline & $0.5,1.0)$ & 100 & 1.93 & 3.43 & 10.36 & 10.91 & 0.895 \\
\hline & & 200 & 1.93 & 3.05 & 7.25 & 7.86 & 0.875 \\
\hline & & 500 & 1.93 & 2.87 & 4.58 & 5.40 & 0.837 \\
\hline & $(0,0.25$ & 50 & 1.93 & 3.96 & 11.84 & 12.48 & 0.875 \\
\hline & $0.375,0.5$ & 100 & 1.93 & 3.37 & 8.25 & 8.91 & 0.864 \\
\hline & $0.75,1.0)$ & 200 & 1.93 & 2.40 & 5.75 & 6.23 & 0.858 \\
\hline & & 500 & 1.93 & 2.41 & 3.63 & 4.36 & 0.833 \\
\hline \multirow{8}{*}{$\begin{array}{c}\text { Convex } \\
(0.01,0.625,1)\end{array}$} & $(0,0.25$ & 50 & 0.56 & 15.53 & 74.75 & 76.35 & 0.893 \\
\hline & $0.5,1.0)$ & 100 & 0.56 & 10.00 & 52.14 & 53.09 & 0.897 \\
\hline & & 200 & 0.56 & 8.10 & 36.85 & 37.73 & 0.907 \\
\hline & & 500 & 0.56 & 6.10 & 23.52 & 24.29 & 0.916 \\
\hline & $(0,0.25$ & 50 & 0.56 & 12.05 & 64.58 & 65.69 & 0.895 \\
\hline & $0.375,0.5$ & 100 & 0.56 & 8.34 & 45.93 & 46.68 & 0.912 \\
\hline & $0.75,1.0)$ & 200 & 0.56 & 8.32 & 32.38 & 33.43 & 0.912 \\
\hline & & 500 & 0.56 & 7.02 & 20.63 & 21.79 & 0.912 \\
\hline \multirow{8}{*}{$\begin{array}{c}\text { Concave } \\
(>0) \\
(0.01,0.875,2)\end{array}$} & $(0,0.25$ & 50 & 2.35 & 9.95 & 31.67 & 33.20 & 0.949 \\
\hline & $0.5,1.0)$ & 100 & 2.35 & 6.66 & 20.90 & 21.93 & 0.962 \\
\hline & & 200 & 2.35 & 5.42 & 14.30 & 15.30 & 0.955 \\
\hline & & 500 & 2.35 & 5.36 & 8.96 & 10.44 & 0.915 \\
\hline & $(0,0.25$ & 50 & 2.35 & 7.49 & 26.08 & 27.13 & 0.955 \\
\hline & $0.375,0.5$ & 100 & 2.35 & 6.05 & 17.64 & 18.65 & 0.964 \\
\hline & $0.75,1.0)$ & 200 & 2.35 & 4.72 & 12.13 & 13.02 & 0.966 \\
\hline & & 500 & 2.35 & 4.83 & 7.63 & 9.03 & 0.925 \\
\hline \multirow{8}{*}{$\begin{array}{c}\text { Concave } \\
(=0) \\
(0.01,1.000,5)\end{array}$} & $(0,0.25$ & 50 & 5.17 & 9.71 & 33.43 & 34.82 & 0.798 \\
\hline & $0.5,1.0)$ & 100 & 5.17 & 6.68 & 17.69 & 18.91 & 0.873 \\
\hline & & 200 & 5.17 & 4.74 & 11.12 & 12.09 & 0.966 \\
\hline & & 500 & 5.17 & 3.28 & 5.45 & 6.36 & 0.953 \\
\hline & $(0,0.25$ & 50 & 5.17 & 2.98 & 11.69 & 12.07 & 0.930 \\
\hline & $0.375,0.5$ & 100 & 5.17 & 2.45 & 8.24 & 8.60 & 0.942 \\
\hline & $0.75,1.0)$ & 200 & 5.17 & 1.76 & 5.80 & 6.06 & 0.954 \\
\hline & & 500 & 5.17 & 1.83 & 3.65 & 4.09 & 0.951 \\
\hline
\end{tabular}

initial slope.

Table A.16: Properties of Estimate of $B M D 10$ based on Additional Risk 


\section{B. APPENDIX OF CHAPTER 3}

\section{B.1 Analytical Method Results}

\section{B.1.1 Preliminary Investigation of Signal-to-Noise Response}

For a selected Hill model parameter values $\left(\alpha_{0}, \kappa_{0}\right.$ and $\left.\eta_{0}\right)$, that of number of doses and that of size per dose in the dose range, 1000 simulations are done and for each 1000 simulation maximum likelihood estimates $(\widehat{\alpha}, \widehat{\kappa}, \widehat{\eta})$ and their variancecovariance $(\operatorname{cov}(\widehat{\alpha}, \widehat{\kappa}, \widehat{\eta}))$ is calculated. Variance-covariance $\left(\operatorname{cov}\left(\alpha_{0}, \kappa_{0}, \eta_{0}\right)\right)$ of Hill model parameters at initial values are obtained by using the inverse of the negative of the second derivatives of the log of the likelihood function (Chapter 2).

In addition to true $\ln \left(S N R\left(d_{i}\right)\right)$ at $d_{1}, \cdots, d_{k}$, three different expectations and variances of $\ln \left(\widehat{S N R}\left(d_{i}\right)\right)$ at $d_{1}, \cdots, d_{k}$ are estimated and compared at dose points 2 , 4 , and 8 .

- For each simulation, expectation and variance of $\ln \left(\widehat{S N R}\left(d_{i}\right)\right)$ is estimated by substituting the analytically obtained variance-covariance and initial Hill model parameter $\left(\alpha_{0}, \kappa_{0}, \eta_{0}\right)$ in Equations 3.23 and 3.14. $\left.\widehat{\widehat{E}}\left(\ln \widehat{S N R}\left(d_{i}\right)\right)\right|_{\theta}$ and $\left.\widehat{\widehat{V a r}}\left(\ln \left(\widehat{S N R}\left(d_{i}\right)\right)\right)\right|_{\theta}$ is obtained by taking average over 1000 estimated expectation and variance of $\ln \left(\widehat{S N R}\left(d_{i}\right)\right)$.

- For each simulation, expectation and variance of $\ln \left(\widehat{S N R}\left(d_{i}\right)\right)$ is estimated by substituting the estimated variance-covariance and MLEs of Hill model parameters in Equations 3.23 and 3.14. $\left.\overline{\widehat{E}}\left(\ln \left(\widehat{S N R}\left(d_{i}\right)\right)\right)\right|_{\widehat{\theta}}$ and $\left.\overline{\widehat{V a r}}\left(\ln \left(\widehat{S N R}\left(d_{i}\right)\right)\right)\right|_{\theta}$ are obtained by taking average over 1000 estimated expectation and variance of $\ln \left(\widehat{S N R}\left(d_{i}\right)\right)$.

- $\ln \left(\widehat{S N R}\left(d_{i}\right)\right)$ is estimated for each simulation using Equation 3.9 Average over $1000 \ln \left(\widehat{S N R}\left(d_{i}\right)\right)$ gives $\ln \left(\widehat{S N R}\left(d_{i}\right)\right)$ and variance of $1000 \ln \left(\widehat{S N R}\left(d_{i}\right)\right)$ 


$$
\text { gives } \operatorname{Var}\left(\ln \left(\widehat{S N R}\left(d_{i}\right)\right)\right) \text {. }
$$

Coverage probabilities (Table B.3) of true SNR at dose points 2, 4 and 8 are estimated using exponentiation and delta method. As the exponentiation gave better coverage, this method is used in Chapter 3.

\begin{tabular}{|c|c|c|c|c|c|c|c|}
\hline \multirow{2}{*}{\multicolumn{2}{|c|}{$d$}} & \multicolumn{6}{|l|}{$n_{i}$} \\
\hline & & 50 & 100 & 200 & 300 & 400 & 500 \\
\hline \multirow{3}{*}{ True $\ln \left(S N R\left(d_{i}\right)\right)$} & 2 & -0.961 & -0.614 & -0.268 & -0.065 & 0.079 & 0.190 \\
\hline & 4 & -0.186 & 0.160 & 0.507 & 0.709 & 0.853 & 0.965 \\
\hline & 8 & 0.529 & 0.876 & 1.222 & 1.425 & 1.569 & 1.681 \\
\hline \multirow{3}{*}{$\left.\widehat{E}\left(\ln \left(\widehat{S N R}\left(d_{i}\right)\right)\right)\right|_{\theta}$} & 2 & -0.621 & -0.564 & -0.180 & -0.023 & 0.110 & 0.213 \\
\hline & 4 & 0.124 & 0.203 & 0.587 & 0.748 & 0.882 & 0.985 \\
\hline & 8 & 0.835 & 0.917 & 1.302 & 1.463 & 1.597 & 1.701 \\
\hline \multirow{3}{*}{$\left.\widehat{E}\left(\ln \left(\widehat{S N R}\left(d_{i}\right)\right)\right)\right|_{\widehat{\theta}}$} & 2 & -1.214 & -0.833 & -0.460 & -0.282 & -0.121 & -0.021 \\
\hline & 4 & 0.121 & 0.148 & 0.457 & 0.641 & 0.785 & 0.888 \\
\hline & 8 & 1.018 & 1.010 & 1.253 & 1.440 & 1.572 & 1.677 \\
\hline \multirow{3}{*}{$\ln \left(\widehat{S N R}\left(d_{i}\right)\right)$} & 2 & -1.684 & -0.999 & -0.531 & -0.326 & -0.154 & -0.047 \\
\hline & 4 & -0.484 & 0.009 & 0.401 & 0.606 & 0.758 & 0.867 \\
\hline & 8 & 0.503 & 0.854 & 1.200 & 1.407 & 1.547 & 1.657 \\
\hline
\end{tabular}

Table B.1: Comparison of Expectations at Dose Points 


\begin{tabular}{|c|c|c|c|c|c|c|c|}
\hline & \multirow{2}{*}{$d_{i}$} & \multicolumn{6}{|l|}{$n_{i}$} \\
\hline & & 50 & 100 & 200 & 300 & 400 & 500 \\
\hline \multirow{3}{*}{$\left.\widehat{\operatorname{Var}}\left(\ln \left(\widehat{S N R}\left(d_{i}\right)\right)\right)\right|_{\theta}$} & 2 & 0.062 & 0.025 & 0.016 & 0.009 & 0.0078 & 0.005 \\
\hline & 4 & 0.018 & 0.008 & 0.005 & 0.003 & 0.002 & 0.002 \\
\hline & 8 & 0.039 & 0.007 & 0.010 & 0.005 & 0.004 & 0.003 \\
\hline \multirow{3}{*}{$\left.\widehat{\operatorname{Var}}\left(\ln \left(\widehat{S N R}\left(d_{i}\right)\right)\right)\right|_{\widehat{\theta}}$} & 2 & 27.57 & 5.13 & 0.047 & 0.029 & 0.020 & 0.016 \\
\hline & 4 & 6.77 & 1.26 & 0.009 & 0.006 & 0.004 & 0.003 \\
\hline & 8 & 0.039 & 0.016 & 0.007 & 0.005 & 0.004 & 0.003 \\
\hline \multirow{3}{*}{$\operatorname{Var}\left(\ln \left(\widehat{S N R}\left(d_{i}\right)\right)\right)$} & 2 & 4.76 & 0.554 & 0.081 & 0.052 & 0.034 & 0.026 \\
\hline & 4 & 0.987 & 0.105 & 0.013 & 0.008 & 0.006 & 0.004 \\
\hline & 8 & 0.025 & 0.013 & 0.006 & 0.004 & 0.003 & 0.002 \\
\hline
\end{tabular}

Table B.2: Comparison of Variances at Dose Points

\begin{tabular}{|c|c|c|c|c|c|c|c|}
\hline & \multirow[t]{2}{*}{$d_{i}$} & \multicolumn{6}{|l|}{$n_{i}$} \\
\hline & & 50 & 100 & 200 & 300 & 400 & 500 \\
\hline \multirow{3}{*}{ TrueSNR $\left(d_{i}\right)$} & 2 & 0.383 & 0.541 & 0.765 & 0.937 & 1.082 & 1.210 \\
\hline & 4 & 0.830 & 1.174 & 1.660 & 2.033 & 2.347 & 2.624 \\
\hline & 8 & 1.698 & 2.401 & 3.395 & 4.159 & 4.802 & 5.369 \\
\hline \multirow{3}{*}{$\widehat{S N R}\left(d_{i}\right)$} & 2 & 0.289 & 0.414 & 0.610 & 0.739 & 0.871 & 0.966 \\
\hline & 4 & 0.707 & 1.035 & 1.502 & 1.840 & 2.140 & 2.385 \\
\hline & 8 & 1.675 & 2.364 & 3.332 & 4.091 & 4.705 & 5.250 \\
\hline \multirow{3}{*}{$c P b\left(d_{i}\right)_{\text {exp }}$} & 2 & 0.822 & 0.771 & 0.698 & 0.552 & 0.498 & 0.436 \\
\hline & 4 & 0.844 & 0.772 & 0.710 & 0.609 & 0.567 & 0.486 \\
\hline & 8 & 0.954 & 0.931 & 0.921 & 0.936 & 0.910 & 0.907 \\
\hline \multirow{3}{*}{$c P b\left(d_{i}\right)_{\text {delta }}$} & 2 & 0.758 & 0.703 & 0.622 & 0.500 & 0.451 & 0.388 \\
\hline & 4 & 0.862 & 0.779 & 0.698 & 0.593 & 0.545 & 0.472 \\
\hline & 8 & 0.979 & 0.972 & 0.936 & 0.939 & 0.910 & 0.901 \\
\hline
\end{tabular}

Table B.3: Results of Coverage Probability of True SNR 


\section{B.1.2 Results of SNCD Estimates}

Tables summarize the results of SNCD estimates all possible combination of selected Hill model parameter combination, numbers of dose and sizes per dose. These results are shown in Figures 3.2, 3.3 and 3.4

\begin{tabular}{|c|c|c|c|c|c|c|}
\hline \multirow[b]{2}{*}{$r=1000$} & \multicolumn{6}{|c|}{$n_{i}(\alpha=0.01, \kappa=4, \eta=3, k=4)$} \\
\hline & 50 & 100 & 200 & 300 & 400 & 500 \\
\hline$S N C D 1_{t r}$ & 2.46 & 1.96 & 1.56 & 1.35 & 1.23 & 1.13 \\
\hline$\widehat{S N C D} L 1_{n}$ & 2.13 & 1.75 & 1.40 & 1.22 & 1.09 & 1.01 \\
\hline$S \widehat{\overline{N C D}} 1_{n}$ & 2.62 & 2.09 & 1.63 & 1.40 & 1.24 & 1.13 \\
\hline$S N \overline{\widehat{C D}} U 1_{n}$ & 3.03 & 2.39 & 1.85 & 1.58 & 1.39 & 1.27 \\
\hline Bias1.0 & 0.158 & 0.131 & 0.076 & 0.044 & 0.015 & $4.3 \mathrm{e}-4$ \\
\hline$c P b 1.0_{L U}$ & 0.857 & 0.818 & 0.801 & 0.800 & 0.816 & 0.810 \\
\hline$C c P b 1.0_{L U}$ & 0.873 & 0.850 & 0.824 & 0.812 & 0.808 & 0.809 \\
\hline$c P b 1.0_{05.95}$ & 0.891 & 0.867 & 0.888 & 0.890 & 0.899 & 0.900 \\
\hline$C c P b 1.0_{05.95}$ & 0.908 & 0.907 & 0.897 & 0.906 & 0.897 & 0.900 \\
\hline$S N C D 0.67_{t r}$ & 1.89 & 1.50 & 1.18 & 1.02 & 0.920 & 0.85 \\
\hline$S N \widehat{C D L} 0.67_{n}$ & 1.57 & 1.27 & 1.00 & 0.86 & 0.76 & 0.70 \\
\hline$S N \widehat{\widehat{C D}} 0.67_{n}$ & 2.06 & 1.60 & 1.20 & 1.01 & 0.88 & 0.80 \\
\hline$S N \widehat{C D U} 0.67_{n}$ & 2.46 & 1.90 & 1.41 & 1.17 & 1.01 & 0.91 \\
\hline Bias 0.67 & 0.163 & 0.0 .098 & 0.207 & -0.013 & -0.038 & -0.05 \\
\hline$c P b 0.67_{L U}$ & 0.840 & 0.839 & 0.814 & 0.784 & 0.734 & 0.686 \\
\hline$C c P b 0.67_{L U}$ & 0.843 & 0.820 & 0.804 & 0.795 & 0.798 & 0.794 \\
\hline$c P b 0.67_{05.95}$ & 0.901 & 0.899 & 0.903 & 0.897 & 0.859 & 0.806 \\
\hline$C c P b 0.67_{05.95}$ & 0.893 & 0.904 & 0.891 & 0.905 & 0.902 & 0.903 \\
\hline
\end{tabular}

Table B.4: SNCDs on Hill Model when $\alpha=0.01, \kappa=4, \eta=3$ and $k=4$ 


\begin{tabular}{|c|c|c|c|c|c|c|}
\hline \multirow[b]{2}{*}{$r=1000$} & \multicolumn{6}{|c|}{$n_{i}(\alpha=0.01, \kappa=4, \eta=3, k=6)$} \\
\hline & 50 & 100 & 200 & 300 & 400 & 500 \\
\hline$S N C D 1_{t r}$ & 2.45 & 1.96 & 1.57 & 1.38 & 1.26 & 1.17 \\
\hline$S \widehat{N C D} L 1_{n}$ & 2.17 & 1.80 & 1.44 & 1.26 & 1.13 & 1.05 \\
\hline$S \widehat{\widehat{N C D}} 1_{n}$ & 2.56 & 2.08 & 1.64 & 1.42 & 1.27 & 1.16 \\
\hline$S N \widehat{C D} U 1_{n}$ & 2.89 & 2.31 & 1.82 & 1.57 & 1.40 & 1.28 \\
\hline Bias 1.0 & 0.116 & 0.112 & 0.064 & 0.039 & 0.009 & -0.006 \\
\hline$c P b 1.0_{L U}$ & 0.883 & 0.824 & 0.818 & 0.842 & 0.866 & 0.836 \\
\hline$C c P b 1.0_{L U}$ & 0.883 & 0.877 & 0.849 & 0.860 & 0.864 & 0.837 \\
\hline$c P b 1.0_{05.95}$ & 0.885 & 0.827 & 0.862 & 0.879 & 0.911 & 0.900 \\
\hline$C c P b 1.0_{05.95}$ & 0.920 & 0.913 & 0.901 & 0.902 & 0.905 & 0.902 \\
\hline$S N C D 0.67_{t r}$ & 1.90 & 1.52 & 1.21 & 1.06 & 0.97 & 0.900 \\
\hline$S N \widehat{C D L} 0.67_{n}$ & 1.60 & 1.31 & 1.04 & 0.90 & 0.800 & 0.73 \\
\hline$S N \widehat{C D} 0.67_{n}$ & 2.02 & 1.60 & 1.22 & 1.04 & 0.91 & 0.83 \\
\hline$S N \widehat{\widehat{C D U}} 0.67_{n}$ & 2.35 & 1.86 & 1.41 & 1.19 & 1.04 & 0.94 \\
\hline Bias0.67 & 0.124 & 0.083 & 0.007 & -0.022 & -0.05 & -0.06 \\
\hline$c P b 0.67_{L U}$ & 0.866 & 0.845 & 0.833 & 0.835 & 0.747 & 0.662 \\
\hline$C c P b 0.67_{L U}$ & 0.863 & 0.867 & 0.837 & 0.850 & 0.856 & 0.831 \\
\hline$c P b 0.67_{05.95}$ & 0.873 & 0.872 & 0.899 & 0.886 & 0.828 & 0.748 \\
\hline$C c P b 0.67_{05.95}$ & 0.903 & 0.904 & 0.895 & 0.904 & 0.914 & 0.906 \\
\hline
\end{tabular}

Table B.5: SNCDs on Hill Model when $\alpha=0.01, \kappa=4, \eta=3$ and $k=6$ 


\begin{tabular}{|c|c|c|c|c|c|c|}
\hline \multirow[b]{2}{*}{$r=1000$} & \multicolumn{6}{|c|}{$n_{i}(\alpha=0.01, \kappa=4, \eta=3, k=8)$} \\
\hline & 50 & 100 & 200 & 300 & 400 & 500 \\
\hline$S N C D 1_{t r}$ & 2.45 & 1.96 & 1.57 & 1.38 & 1.26 & 1.17 \\
\hline$S N \widehat{C D} L 1_{n}$ & 2.18 & 1.79 & 1.44 & 1.26 & 1.13 & 1.05 \\
\hline$S \widehat{N C D} 1_{n}$ & 2.57 & 2.07 & 1.64 & 1.41 & 1.26 & 1.16 \\
\hline$S N \widehat{C D U} U 1_{n}$ & 2.88 & 2.30 & 1.82 & 1.56 & 1.40 & 1.28 \\
\hline Bias1.0 & 0.117 & 0.106 & 0.063 & 0.032 & 0.006 & -0.005 \\
\hline$c P b 1.0_{L U}$ & 0.854 & 0.848 & 0.830 & 0.873 & 0.852 & 0.848 \\
\hline$C c P b 1.0_{L U}$ & 0.883 & 0.889 & 0.867 & 0.876 & 0.848 & 0.852 \\
\hline$c P b 1.0_{05.95}$ & 0.871 & 0.861 & 0.854 & 0.908 & 0.898 & 0.891 \\
\hline$C c P b 1.0_{05.95}$ & 0.910 & 0.926 & 0.912 & 0.909 & 0.897 & 0.885 \\
\hline$S N C D 0.67_{t r}$ & 1.90 & 1.52 & 1.21 & 1.06 & 0.96 & 0.90 \\
\hline$S N \widehat{C D L} 0.67_{n}$ & 1.61 & 1.31 & 1.04 & 0.89 & 0.80 & 0.74 \\
\hline$S N \widehat{C D} 0.67_{n}$ & 2.02 & 1.60 & 1.22 & 1.03 & 0.91 & 0.83 \\
\hline$S N \widehat{C D U} 0.67_{n}$ & 2.35 & 1.85 & 1.40 & 1.18 & 1.03 & 0.94 \\
\hline Bias0.67 & 0.124 & 0.076 & 0.006 & -0.030 & -0.054 & -0.063 \\
\hline$c P b 0.67_{L U}$ & 0.852 & 0.863 & 0.858 & 0.834 & 0.750 & 0.668 \\
\hline$C c P b 0.67_{L U}$ & 0.872 & 0.876 & 0.851 & 0.876 & 0.846 & 0.845 \\
\hline$c P b 0.67_{05.95}$ & 0.859 & 0.881 & 0.911 & 0.878 & 0.808 & 0.732 \\
\hline$C c P b 0.67_{05.95}$ & 0.899 & 0.905 & 0.916 & 0.912 & 0.897 & 0.895 \\
\hline
\end{tabular}

Table B.6: SNCDs on Hill Model when $\alpha=0.01, \kappa=4, \eta=3$ and $k=8$ 


\begin{tabular}{|c|c|c|c|c|c|c|}
\hline \multirow[b]{2}{*}{$r=1000$} & \multicolumn{6}{|c|}{$n_{i}(\alpha=0.01, \kappa=5, \eta=1, k=4)$} \\
\hline & 50 & 100 & 200 & 300 & 400 & 500 \\
\hline$S N C D 1_{t r}$ & 1.33 & 0.87 & 0.59 & 0.47 & 0.40 & 0.36 \\
\hline$S \widehat{N C D} L 1_{n}$ & 1.19 & 0.87 & .62 & 0.52 & 0.45 & 0.40 \\
\hline$S \widehat{\widehat{N C D}} D 1_{n}$ & 1.52 & 1.01 & 0.69 & 0.56 & 0.48 & 0.43 \\
\hline$S N \widehat{\widehat{C D}} U 1_{n}$ & 1.95 & 1.19 & 0.77 & 0.61 & 0.52 & 0.46 \\
\hline Bias1.0 & 0.188 & 0.145 & 0.108 & 0.095 & 0.082 & 0.076 \\
\hline$c P b 1.0_{L U}$ & 0.708 & 0.528 & 0.250 & 0.100 & 0.042 & 0.014 \\
\hline$C c P b 1.0_{L U}$ & 0.758 & 0.719 & 0.715 & 0.714 & 0.740 & 0.729 \\
\hline$c P b 1.0_{05.95}$ & 0.900 & 0.869 & 0.572 & 0.278 & 0.144 & 0.053 \\
\hline$C c P b 1.0_{05.95}$ & 0.867 & 0.910 & 0.902 & 0.885 & 0.906 & 0.915 \\
\hline$S N C D 0.67_{t r}$ & 0.82 & 0.55 & 0.38 & 0.31 & 0.26 & 0.23 \\
\hline$S N \widehat{C D L} 0.67_{n}$ & 0.78 & 0.57 & 0.42 & 0.34 & 0.30 & 0.27 \\
\hline$S N \widehat{C D} 0.67_{n}$ & 0.98 & 0.67 & 0.46 & 0.38 & 0.32 & 0.29 \\
\hline$S N \widehat{C D U} 0.67_{n}$ & 1.23 & 0.78 & 0.51 & 0.41 & 0.35 & 0.31 \\
\hline Bias0.67 & 0.163 & 0.116 & 0.082 & 0.070 & 0.060 & 0.055 \\
\hline$c P b 0.67_{L U}$ & 0.620 & 0.405 & 0.163 & 0.057 & 0.021 & 0.006 \\
\hline$C c P b 0.67_{L U}$ & 0.735 & 0.709 & 0.709 & 0.712 & 0.739 & 0.728 \\
\hline$c P b 0.67_{05.95}$ & 0.945 & 0.807 & 0.439 & 0.178 & 0.082 & 0.024 \\
\hline$C c P b 0.67_{05.95}$ & 0.884 & 0.910 & 0.902 & 0.885 & 0.904 & 0.916 \\
\hline
\end{tabular}

Table B.7: SNCDs on Hill Model when $\alpha=0.01, \kappa=5, \eta=1$ and $k=4$ 


\begin{tabular}{|c|c|c|c|c|c|c|}
\hline \multirow[b]{2}{*}{$r=1000$} & \multicolumn{6}{|c|}{$n_{i}(\alpha=0.01, \kappa=5, \eta=1, k=6)$} \\
\hline & 50 & 100 & 200 & 300 & 400 & 500 \\
\hline$S N C D 1_{t r}$ & 1.26 & 0.81 & 0.54 & 0.43 & 0.36 & 0.32 \\
\hline$S \widehat{N C D} L 1_{n}$ & 1.18 & 0.85 & 0.61 & 0.50 & 0.44 & 0.39 \\
\hline$S \widehat{\overline{N C D}} D 1_{n}$ & 1.47 & 0.98 & 0.67 & 0.54 & 0.47 & 0.42 \\
\hline$S N C D U 1_{n}$ & 1.84 & 1.13 & 0.74 & 0.59 & 0.50 & 0.45 \\
\hline Bias1.0 & 0.202 & 0.170 & 0.137 & 0.118 & 0.104 & 0.097 \\
\hline$c P b 1.0_{L U}$ & 0.657 & 0.373 & 0.062 & 0.003 & 0.000 & 0.000 \\
\hline$C c P b 1.0_{L U}$ & 0.766 & 0.717 & 0.715 & 0.723 & 0.722 & 0.730 \\
\hline$c P b 1.0_{05.95}$ & 0.887 & 0.718 & 0.226 & 0.043 & 0.008 & 0.001 \\
\hline$C c P b 1.0_{05.95}$ & 0.893 & 0.910 & 0.889 & 0.898 & 0.903 & 0.907 \\
\hline$S N C D 0.67_{t r}$ & 0.75 & 0.50 & 0.34 & 0.28 & 0.24 & 0.21 \\
\hline$S N \widehat{C D L} 0.67_{n}$ & 0.76 & 0.56 & 0.41 & 0.34 & 0.29 & 0.26 \\
\hline$S N \widehat{C D} 0.67_{n}$ & 0.94 & 0.64 & 0.45 & 0.36 & 0.31 & 0.28 \\
\hline$S N \widehat{C D U} 0.67_{n}$ & 1.16 & 0.74 & 0.49 & 0.39 & 0.33 & 0.30 \\
\hline Bias0.67 & 0.187 & 0.137 & 0.103 & 0.087 & 0.075 & 0.069 \\
\hline$c P b 0.67_{L U}$ & 0.480 & 0.207 & 0.022 & 0.001 & 0.000 & 0.000 \\
\hline$C c P b 0.67_{L U}$ & 0.751 & 0.709 & 0.712 & 0.720 & 0.720 & 0.729 \\
\hline$c P b 0.67_{05.95}$ & 0.883 & 0.566 & 0.118 & 0.011 & 0.002 & 0.000 \\
\hline$C c P b 0.67_{05.95}$ & 0.911 & 0.913 & 0.890 & 0.898 & 0.902 & 0.907 \\
\hline
\end{tabular}

Table B.8: SNCDs on Hill Model when $\alpha=0.01, \kappa=5, \eta=1$ and $k=6$ 


\begin{tabular}{|c|c|c|c|c|c|c|}
\hline \multirow[b]{2}{*}{$r=1000$} & \multicolumn{6}{|c|}{$n_{i}(\alpha=0.01, \kappa=5, \eta=1, k=8)$} \\
\hline & 50 & 100 & 200 & 300 & 400 & 500 \\
\hline$S N C D 1_{t r}$ & 1.27 & 0.81 & 0.54 & 0.43 & 0.36 & 0.32 \\
\hline$S \widehat{N C D} L 1_{n}$ & 1.18 & 0.85 & 0.61 & 0.51 & 0.44 & 0.40 \\
\hline$S \widehat{S C D} D 1_{n}$ & 1.47 & 0.98 & 0.67 & 0.55 & 0.47 & 0.42 \\
\hline$S N \widehat{\widehat{C D}} U 1_{n}$ & 1.83 & 1.13 & 0.74 & 0.59 & 0.50 & 0.45 \\
\hline Bias1.0 & 0.200 & 0.177 & 0.137 & 0.120 & 0.106 & 0.098 \\
\hline$c P b 1.0_{L U}$ & 0.654 & 0.314 & 0.047 & 0.001 & 0.000 & 0.000 \\
\hline$C c P b 1.0_{L U}$ & 0.765 & 0.746 & 0.748 & 0.716 & 0.746 & 0.728 \\
\hline$c P b 1.0_{05.95}$ & 0.893 & 0.679 & 0.193 & 0.034 & 0.002 & 0.000 \\
\hline$C c P b 1.0_{05.95}$ & 0.894 & 0.910 & 0.919 & 0.891 & 0.908 & 0.914 \\
\hline$S N C D 0.67_{t r}$ & 0.75 & 0.50 & 0.34 & 0.28 & 0.24 & 0.21 \\
\hline$\widehat{S N \widehat{C D L} 0.67_{n}}$ & 0.76 & 0.56 & 0.41 & 0.34 & 0.29 & 0.26 \\
\hline$S N \widehat{C D} 0.67_{n}$ & 0.94 & 0.65 & 0.45 & 0.36 & 0.31 & 0.28 \\
\hline$S N \widehat{C D U} 0.67_{n}$ & 1.15 & 0.74 & 0.49 & 0.39 & 0.33 & 0.30 \\
\hline Bias0.67 & 0.184 & 0.142 & 0.103 & 0.088 & 0.077 & 0.070 \\
\hline$c P b 0.67_{L U}$ & 0.488 & 0.190 & 0.022 & 0.001 & 0.000 & 0.000 \\
\hline$C c P b 0.67_{L U}$ & 0.749 & 0.739 & 0.744 & 0.712 & 0.742 & 0.727 \\
\hline$c P b 0.67_{05.95}$ & 0.891 & 0.500 & 0.081 & 0.006 & 0.001 & 0.000 \\
\hline$C c P b 0.67_{05.95}$ & 0.905 & 0.915 & 0.919 & 0.891 & 0.908 & 0.913 \\
\hline
\end{tabular}

Table B.9: SNCDs on Hill Model when $\alpha=0.01, \kappa=5, \eta=1$ and $k=8$ 


\begin{tabular}{|c||c|c|c|c|c|c|}
\hline \multicolumn{1}{|c||}{} & \multicolumn{5}{c|}{$n_{i}(\alpha=0.01, \kappa=7, \eta=2, k=4)$} \\
\cline { 2 - 7 }$r=1000$ & 50 & 100 & 200 & 300 & 400 & 500 \\
\hline \hline$S N C D 1_{t r}$ & 3.36 & 2.42 & 1.75 & 1.44 & 1.25 & 1.13 \\
\hline$S N \widehat{\widehat{C D}} L 1_{n}$ & 2.75 & 2.14 & 1.63 & 1.39 & 1.23 & 1.12 \\
$S \widehat{\widehat{N C D}} 1_{n}$ & 3.64 & 2.70 & 1.98 & 1.65 & 1.43 & 1.29 \\
$S N \widehat{\widehat{C D}} U 1_{n}$ & 4.42 & 3.18 & 2.31 & 1.91 & 1.65 & 1.48 \\
\hline Bias1.0 & 0.279 & 0.274 & 0.233 & 0.209 & 0.177 & 0.168 \\
\hline$c P b 1.0_{L U}$ & 0.877 & 0.801 & 0.707 & 0.641 & 0.604 & 0.542 \\
$C c P b 1.0_{L U}$ & 0.873 & 0.838 & 0.793 & 0.794 & 0.795 & 0.797 \\
\hline$c P b 1.0_{05.95}$ & 0.894 & 0.851 & 0.802 & 0.783 & 0.789 & 0.739 \\
$C c P b 1.0_{05.95}$ & 0.923 & 0.905 & 0.891 & 0.888 & 0.894 & 0.895 \\
\hline \hline$S N C D 0.67_{t r}$ & 2.30 & 1.66 & 1.19 & 0.98 & 0.85 & 0.76 \\
\hline$S N \widehat{\widehat{C D L} 0.67_{n}}$ & 1.81 & 1.44 & 1.10 & 0.94 & 0.83 & 0.76 \\
$S N \widehat{\widehat{C D}} 0.67_{n}$ & 2.63 & 1.94 & 1.39 & 1.14 & 0.98 & 0.88 \\
$S N \widehat{\widehat{C D U}} 0.67_{n}$ & 3.28 & 2.39 & 1.69 & 1.36 & 1.15 & 1.03 \\
\hline$B i a s 0.67$ & 0.325 & 0.282 & 0.197 & 0.161 & 0.129 & 0.119 \\
\hline$c P b 0.67_{L U}$ & 0.836 & 0.785 & 0.709 & 0.660 & 0.631 & 0.575 \\
$C c P b 0.67_{L U}$ & 0.842 & 0.810 & 0.775 & 0.771 & 0.792 & 0.788 \\
\hline$c P b 0.67_{05.95}$ & 0.873 & 0.852 & 0.866 & 0.872 & 0.856 & 0.821 \\
$C c P b 0.67_{05.95}$ & 0.893 & 0.887 & 0.886 & 0.889 & 0.908 & 0.896 \\
\hline \hline
\end{tabular}

Table B.10: SNCDs on Hill Model when $\alpha=0.01, \kappa=7, \eta=2$ and $k=4$ 


\begin{tabular}{|c||c|c|c|c|c|c|}
\hline \multicolumn{1}{|c||}{} & \multicolumn{5}{c|}{$n_{i}(\alpha=0.01, \kappa=7, \eta=2, k=6)$} \\
\cline { 2 - 7 }$r=1000$ & 50 & 100 & 200 & 300 & 400 & 500 \\
\hline \hline$S N C D 1_{t r}$ & 3.34 & 2.41 & 1.75 & 1.45 & 1.27 & 1.15 \\
\hline$S N \widehat{\widehat{C D}} L 1_{n}$ & 2.88 & 2.18 & 1.66 & 1.41 & 1.24 & 1.14 \\
$S \widehat{\widehat{N C D}} 1_{n}$ & 3.60 & 2.64 & 1.97 & 1.65 & 1.43 & 1.30 \\
$S N \widehat{\widehat{C D}} U 1_{n}$ & 4.25 & 3.04 & 2.24 & 1.88 & 1.62 & 1.47 \\
\hline Bias 1.0 & 0.253 & 0.230 & 0.213 & 0.194 & 0.157 & 0.156 \\
\hline$c P b 1.0_{L U}$ & 0.878 & 0.792 & 0.708 & 0.634 & 0.657 & 0.554 \\
$C c P b 1.0_{L U}$ & 0.910 & 0.855 & 0.846 & 0.849 & 0.842 & 0.841 \\
\hline$c P b 1.0_{05.95}$ & 0.894 & 0.836 & 0.760 & 0.708 & 0.755 & 0.683 \\
$C c P b 1.0_{05.95}$ & 0.932 & 0.910 & 0.901 & 0.904 & 0.901 & 0.891 \\
\hline \hline$S N C D 0.67_{t r}$ & 2.30 & 1.67 & 1.21 & 1.00 & 0.87 & 0.79 \\
\hline$S N \widehat{\widehat{C D L} 0.67_{n}}$ & 1.87 & 1.47 & 1.12 & 0.95 & 0.84 & 0.77 \\
$S N \widehat{\widehat{C D}} 0.67_{n}$ & 2.56 & 1.91 & 1.39 & 1.15 & 0.99 & 0.89 \\
$S N \widehat{\widehat{C D U}} 0.67_{n}$ & 3.11 & 2.29 & 1.66 & 1.35 & 0.115 & 1.03 \\
\hline$B i a s 0.67$ & 0.268 & 0.238 & 0.179 & 0.146 & 0.110 & 0.107 \\
\hline$c P b 0.67_{L U}$ & 0.862 & 0.784 & 0.743 & 0.674 & 0.707 & 0.625 \\
$C c P b 0.67_{L U}$ & 0.866 & 0.840 & 0.831 & 0.832 & 0.833 & 0.835 \\
\hline$c P b 0.67_{05.95}$ & 0.885 & 0.828 & 0.834 & 0.809 & 0.835 & 0.779 \\
$C c P b 0.67_{05.95}$ & 0.915 & 0.890 & 0.893 & 0.904 & 0.905 & 0.891 \\
\hline \hline
\end{tabular}

Table B.11: SNCDs on Hill Model when $\alpha=0.01, \kappa=7, \eta=2$ and $k=6$ 


\begin{tabular}{|c||c|c|c|c|c|c|}
\hline \multicolumn{1}{|c||}{} & \multicolumn{5}{c|}{$n_{i}(\alpha=0.01, \kappa=7, \eta=2, k=8)$} \\
\cline { 2 - 7 }$r=1000$ & 50 & 100 & 200 & 300 & 400 & 500 \\
\hline \hline$S N C D 1_{t r}$ & 3.34 & 2.41 & 1.75 & 1.45 & 1.27 & 1.15 \\
\hline$S N \widehat{\widehat{C D}} L 1_{n}$ & 2.93 & 2.20 & 1.65 & 1.40 & 1.24 & 1.14 \\
$S \widehat{\widehat{N C D}} 1_{n}$ & 3.60 & 2.65 & 1.94 & 1.64 & 1.43 & 1.30 \\
$S N \widehat{\widehat{C D}} U 1_{n}$ & 4.17 & 3.03 & 2.21 & 1.86 & 1.62 & 1.46 \\
\hline Bias1.0 & 0.258 & 0.240 & 0.188 & 0.182 & 0.154 & 0.148 \\
\hline$c P b 1.0_{L U}$ & 0.856 & 0.796 & 0.729 & 0.648 & 0.639 & 0.571 \\
$C c P b 1.0_{L U}$ & 0.889 & 0.885 & 0.846 & 0.854 & 0.856 & 0.854 \\
\hline$c P b 1.0_{05.95}$ & 0.874 & 0.830 & 0.767 & 0.733 & 0.751 & 0.687 \\
$C c P b 1.0_{05.95}$ & 0.935 & 0.931 & 0.907 & 0.904 & 0.912 & 0.901 \\
\hline \hline$S N C D 0.67_{t r}$ & 2.30 & 1.67 & 1.21 & 1.00 & 0.87 & 0.79 \\
\hline$S N \widehat{\widehat{C D L} 0.67_{n}}$ & 1.90 & 1.48 & 1.11 & 0.95 & 0.84 & 0.77 \\
$S N \widehat{\widehat{C D}} 0.67_{n}$ & 2.57 & 1.92 & 1.37 & 1.13 & 0.98 & 0.89 \\
$S N \widehat{\widehat{C D U}} 0.67_{n}$ & 3.09 & 2.29 & 1.62 & 1.34 & 1.14 & 1.02 \\
\hline$B i a s 0.67$ & 0.277 & 0.248 & 0.155 & 0.135 & 0.107 & 0.159 \\
\hline$c P b 0.67_{L U}$ & 0.849 & 0.810 & 0.757 & 0.709 & 0.705 & 0.638 \\
$C c P b 0.67_{L U}$ & 0.840 & 0.869 & 0.832 & 0.847 & 0.855 & 0.845 \\
\hline$c P b 0.67_{05.95}$ & 0.867 & 0.830 & 0.847 & 0.831 & 0.850 & 0.778 \\
$C c P b 0.67_{05.95}$ & 0.911 & 0.908 & 0.910 & 0.905 & 0.916 & 0.905 \\
\hline \hline
\end{tabular}

Table B.12: SNCDs on Hill Model when $\alpha=0.01, \kappa=7, \eta=2$ and $k=8$ 


\begin{tabular}{|c||c|c|c|c|c|c|}
\hline \multicolumn{1}{|c||}{} & \multicolumn{5}{c|}{$n_{i}(\alpha=0.01, \kappa=8, \eta=5, k=4)$} \\
\cline { 2 - 7 }$r=1000$ & 50 & 100 & 200 & 300 & 400 & 500 \\
\hline \hline$S N C D 1_{t r}$ & 5.99 & 5.26 & 4.63 & 4.31 & 4.10 & 3.95 \\
\hline$S N \widehat{\widehat{C D}} L 1_{n}$ & 5.11 & 4.61 & 4.24 & 4.08 & 3.96 & 3.88 \\
$S \widehat{\widehat{N C D}} 1_{n}$ & 6.00 & 5.34 & 4.81 & 4.53 & 4.35 & 4.20 \\
$S N \widehat{\widehat{C D}} U 1_{n}$ & 6.60 & 5.80 & 5.14 & 4.83 & 4.62 & 4.45 \\
\hline Bias 1.0 & 0.014 & 0.086 & 0.173 & 0.214 & 0.245 & 0.256 \\
\hline$c P b 1.0_{L U}$ & 0.782 & 0.797 & 0.875 & 0.830 & 0.703 & 0.622 \\
$C c P b 1.0_{L U}$ & 0.777 & 0.765 & 0.785 & 0.817 & 0.843 & 0.756 \\
\hline$c P b 1.0_{05.95}$ & 0.838 & 0.794 & 0.796 & 0.813 & 0.807 & 0.761 \\
$C c P b 1.0_{05.95}$ & 0.845 & 0.802 & 0.809 & 0.859 & 0.889 & 0.874 \\
\hline \hline$S N C D 0.67_{t r}$ & 5.16 & 4.55 & 4.02 & 3.76 & 3.58 & 3.46 \\
\hline$S N \widehat{\widehat{C D L} 0.67_{n}}$ & 4.40 & 4.01 & 3.72 & 3.60 & 3.51 & 3.45 \\
$S N \widehat{\widehat{C D}} 0.67_{n}$ & 5.29 & 4.76 & 4.31 & 4.07 & 3.92 & 3.79 \\
$S N \widehat{\widehat{C D U}} 0.67_{n}$ & 5.82 & 5.19 & 4.65 & 4.38 & 4.20 & 4.05 \\
\hline$B i a s 0.67$ & 0.133 & 0.212 & 0.287 & 0.313 & 0.332 & 0.332 \\
\hline$c P b 0.67_{L U}$ & 0.672 & 0.799 & 0.885 & 0.688 & 0.589 & 0.524 \\
$C c P b 0.67_{L U}$ & 0.628 & 0.719 & 0.755 & 0.791 & 0.806 & 0.712 \\
\hline$c P b 0.67_{05.95}$ & 0.715 & 0.770 & 0.777 & 0.782 & 0.776 & 0.736 \\
$C c P b 0.67_{05.95}$ & 0.747 & 0.790 & 0.810 & 0.855 & 0.886 & 0.866 \\
\hline \hline
\end{tabular}

Table B.13: SNCDs on Hill Model when $\alpha=0.01, \kappa=8, \eta=5$ and $k=4$ 


\begin{tabular}{|c||c|c|c|c|c|c|}
\hline \multicolumn{1}{|c||}{} & \multicolumn{5}{c|}{$n_{i}(\alpha=0.01, \kappa=8, \eta=5, k=6)$} \\
\cline { 2 - 7 }$r=1000$ & 50 & 100 & 200 & 300 & 400 & 500 \\
\hline \hline$S N C D 1_{t r}$ & 5.95 & 5.22 & 4.61 & 4.29 & 4.09 & 3.95 \\
\hline$S N \widehat{\widehat{C D}} L 1_{n}$ & 5.64 & 5.13 & 4.47 & 4.21 & 4.03 & 3.92 \\
$S \widehat{\widehat{N C D}} 1_{n}$ & 6.11 & 5.50 & 4.77 & 4.47 & 4.26 & 4.13 \\
$S N \widehat{\widehat{C D}} U 1_{n}$ & 6.57 & 5.80 & 5.00 & 4.68 & 4.46 & 4.30 \\
\hline Bias 1.0 & 0.152 & 0.274 & 0.159 & 0.175 & 0.170 & 0.176 \\
\hline$c P b 1.0_{L U}$ & 0.789 & 0.700 & 0.674 & 0.618 & 0.617 & 0.583 \\
$C c P b 1.0_{L U}$ & 0.826 & 0.800 & 0.717 & 0.698 & 0.709 & 0.712 \\
\hline$c P b 1.0_{05.95}$ & 0.855 & 0.800 & 0.831 & 0.811 & 0.800 & 0.763 \\
$C c P b 1.0_{05.95}$ & 0.885 & 0.800 & 0.886 & 0.885 & 0.895 & 0.898 \\
\hline \hline$S N C D 0.67_{t r}$ & 5.12 & 4.52 & 4.02 & 3.78 & 3.62 & 3.50 \\
\hline$S N \widehat{\widehat{C D L} 0.67_{n}}$ & 4.79 & 4.38 & 3.85 & 3.67 & 3.54 & 3.46 \\
$S N \widehat{\widehat{C D}} 0.67_{n}$ & 5.35 & 4.84 & 4.20 & 3.96 & 3.79 & 3.68 \\
$S N \widehat{\widehat{C D U}} 0.67_{n}$ & 5.77 & 5.16 & 4.46 & 4.19 & 4.00 & 3.87 \\
\hline$B i a s 0.67$ & 0.232 & 0.320 & 0.173 & 0.184 & 0.175 & 0.182 \\
\hline$c P b 0.67_{L U}$ & 0.704 & 0.700 & 0.692 & 0.641 & 0.630 & 0.586 \\
$C c P b 0.67_{L U}$ & 0.702 & 0.800 & 0.714 & 0.721 & 0.714 & 0.728 \\
\hline$c P b 0.67_{05.95}$ & 0.815 & 0.800 & 0.850 & 0.821 & 0.806 & 0.760 \\
$C c P b 0.67_{05.95}$ & 0.865 & 0.900 & 0.885 & 0.889 & 0.906 & 0.904 \\
\hline \hline
\end{tabular}

Table B.14: SNCDs on Hill Model when $\alpha=0.01, \kappa=8, \eta=5$ and $k=6$ 


\begin{tabular}{|c||c|c|c|c|c|c|}
\hline \multicolumn{1}{|c||}{} & \multicolumn{5}{c|}{$n_{i}(\alpha=0.01, \kappa=8, \eta=5, k=8)$} \\
\cline { 2 - 7 }$r=1000$ & 50 & 100 & 200 & 300 & 400 & 500 \\
\hline \hline$S N C D 1_{t r}$ & 5.95 & 5.22 & 4.61 & 4.29 & 4.09 & 3.95 \\
\hline$S N \widehat{\widehat{C D}} L 1_{n}$ & 5.68 & 5.05 & 4.49 & 4.22 & 4.05 & 3.93 \\
$S \widehat{\widehat{N C D}} 1_{n}$ & 6.06 & 5.34 & 4.73 & 4.43 & 4.23 & 4.10 \\
$S N \widehat{\widehat{C D}} U 1_{n}$ & 6.44 & 5.59 & 4.92 & 4.61 & 4.39 & 4.24 \\
\hline Bias1.0 & 0.108 & 0.119 & 0.119 & 0.136 & 0.141 & 0.150 \\
\hline$c P b 1.0_{L U}$ & 0.808 & 0.739 & 0.671 & 0.639 & 0.595 & 0.556 \\
$C c P b 1.0_{L U}$ & 0.835 & 0.769 & 0.725 & 0.712 & 0.727 & 0.726 \\
\hline$c P b 1.0_{05.95}$ & 0.859 & 0.857 & 0.836 & 0.791 & 0.768 & 0.723 \\
$C c P b 1.0_{05.95}$ & 0.888 & 0.898 & 0.880 & 0.889 & 0.907 & 0.889 \\
\hline \hline$S N C D 0.67_{t r}$ & 5.12 & 4.52 & 4.02 & 3.78 & 3.62 & 3.50 \\
\hline$S N \widehat{\widehat{C D L} 0.67_{n}}$ & 4.81 & 4.31 & 3.88 & 3.69 & 3.56 & 3.47 \\
$S N \widehat{\widehat{C D}} 0.67_{n}$ & 5.26 & 4.67 & 4.16 & 3.93 & 3.77 & 3.66 \\
$S N \widehat{\widehat{C D U}} 0.67_{n}$ & 5.60 & 4.94 & 4.39 & 4.12 & 3.95 & 3.83 \\
\hline$B i a s 0.67$ & 0.136 & 0.150 & 0.141 & 0.154 & 0.154 & 0.162 \\
\hline$c P b 0.67_{L U}$ & 0.736 & 0.727 & 0.688 & 0.641 & 0.597 & 0.560 \\
$C c P b 0.67_{L U}$ & 0.747 & 0.751 & 0.723 & 0.719 & 0.732 & 0.730 \\
\hline$c P b 0.67_{05.95}$ & 0.853 & 0.859 & 0.831 & 0.794 & 0.773 & 0.721 \\
$C c P b 0.67_{05.95}$ & 0.880 & 0.898 & 0.875 & 0.893 & 0.902 & 0.889 \\
\hline \hline
\end{tabular}

Table B.15: SNCDs on Hill Model when $\alpha=0.01, \kappa=8, \eta=5$ and $k=8$ 


\section{B.2 Bootstrap Method}

\section{B.2.1 Parametric Bootstrap Method Results}

Tables summarize the results of SNCD estimates all possible combination of selected Hill model parameter combination, numbers of dose and sizes per dose when using parametric bootstrap method. These results are shown in Figures 3.5, 3.6 and 3.7

\begin{tabular}{|c|c|c|c|c|c|c|}
\hline \multirow[b]{2}{*}{$r=1000$} & \multicolumn{6}{|c|}{$n_{i}(\alpha=0.01, \kappa=4, \eta=3, k=4)$} \\
\hline & 50 & 100 & 200 & 300 & 400 & 500 \\
\hline$S N C D 1_{t r}$ & 2.45 & 1.96 & 1.56 & 1.35 & 1.23 & 1.13 \\
\hline$\widehat{S N C D} L 1_{n}$ & 1.86 & 1.52 & 1.26 & 1.12 & 1.02 & 0.96 \\
\hline$\widehat{S \widehat{N C D}} D 1_{n}$ & 2.47 & 1.94 & 1.55 & 1.35 & 1.22 & 1.13 \\
\hline$S N \widehat{\overline{C D}} U 1_{n}$ & 3.10 & 2.39 & 1.88 & 1.61 & 1.50 & 1.33 \\
\hline Bias1.0 & 0.017 & -0.017 & -0.002 & -0.007 & -0.003 & -0.002 \\
\hline$c P b 1.0_{L U}$ & 0.963 & 0.911 & 0.842 & 0.828 & 0.792 & 0.764 \\
\hline$C c P b 1.0_{L U}$ & 0.957 & 0.910 & 0.844 & 0.823 & 0.792 & 0.766 \\
\hline$c P b 1.0_{05.95}$ & 0.900 & 0.879 & 0.877 & 0.886 & 0.868 & 0.879 \\
\hline$C c P b 1.0_{05.95}$ & 0.900 & 0.878 & 0.878 & 0.887 & 0.868 & 0.881 \\
\hline$S N C D 0.67_{t r}$ & 1.89 & 1.50 & 1.18 & 1.02 & 0.92 & 0.85 \\
\hline$\widehat{S N C \widehat{C D L} 0.67_{n}}$ & 1.31 & 1.09 & 0.92 & 0.81 & 0.74 & 0.69 \\
\hline$S N \widehat{\widehat{C D}} 0.67_{n}$ & 1.91 & 1.49 & 1.19 & 1.02 & 0.93 & 0.85 \\
\hline$S N \widehat{\widehat{C D U}} 0.67_{n}$ & 2.55 & 1.98 & 1.57 & 1.34 & 1.15 & 1.11 \\
\hline Bias0.67 & 0.017 & -0.010 & 0.008 & 0.002 & 0.004 & 0.005 \\
\hline$c P b 0.67_{L U}$ & 0.929 & 0.853 & 0.781 & 0.774 & 0.749 & 0.732 \\
\hline$C c P b 0.67_{L U}$ & 0.926 & 0.851 & 0.778 & 0.778 & 0.750 & 0.725 \\
\hline$c P b 0.67_{05.95}$ & 0.875 & 0.861 & 0.866 & 0.881 & 0.861 & 9,873 \\
\hline$C c P b 0.67_{05.95}$ & 0.875 & 0.860 & 0.864 & 0.882 & 0.864 & 0.868 \\
\hline
\end{tabular}

Table B.16: SNCDs on Hill Model when $\alpha=0.01, \kappa=4, \eta=3$ and $k=4$ 


\begin{tabular}{|c|c|c|c|c|c|c|}
\hline \multirow[b]{2}{*}{$r=1000$} & \multicolumn{6}{|c|}{$n_{i}(\alpha=0.01, \kappa=4, \eta=3, k=6)$} \\
\hline & 50 & 100 & 200 & 300 & 400 & 500 \\
\hline$S N C D 1_{t r}$ & 2.43 & 1.96 & 1.57 & 1.38 & 1.26 & 1.17 \\
\hline$S N \widehat{C D} L 1_{n}$ & 1.74 & 1.43 & 1.21 & 1.10 & 1.01 & 0.95 \\
\hline$S \widehat{N C D} 1_{n}$ & 2.42 & 1.87 & 1.50 & 1.34 & 1.22 & 1.13 \\
\hline$S N \widehat{C D} U 1_{n}$ & 2.99 & 2.36 & 2.02 & 1.60 & 1.45 & 1.55 \\
\hline Bias1.0 & -0.011 & -0.093 & -0.072 & -0.044 & -0.038 & -0.036 \\
\hline$c P b 1.0_{L U}$ & 0.952 & 0.912 & 0.684 & 0.635 & 0.548 & 0.529 \\
\hline$C c P b 1.0_{L U}$ & 0.955 & 0.864 & 0.632 & 0.616 & 0.533 & 0.528 \\
\hline$c P b 1.0_{05.95}$ & 0.914 & 0.903 & 0.845 & 0.849 & 0.808 & 0.822 \\
\hline$C c P b 1.0_{05.95}$ & 0.909 & 0.860 & 0.817 & 0.839 & 0.796 & 0.817 \\
\hline$S N C D 0.67_{t r}$ & 1.89 & 1.52 & 1.22 & 1.06 & 0.97 & -0.015 \\
\hline$S N \widehat{C D L} 0.67_{n}$ & 1.21 & 1.04 & 0.91 & 0.83 & 0.77 & 0.71 \\
\hline$S N \widehat{C D} 0.67_{n}$ & 1.79 & 1.41 & 1.17 & 1.04 & 0.96 & 0.88 \\
\hline$S N \widehat{C D U} 0.67_{n}$ & 2.46 & 1.87 & 1.48 & 1.30 & 1.18 & 1.09 \\
\hline Bias0.67 & -0.106 & -0.110 & -0.050 & -0.021 & -0.010 & -0.015 \\
\hline$c P b 0.67_{L U}$ & 0.904 & 0.701 & 0.526 & 0.534 & 0.473 & 0.455 \\
\hline$C c P b 0.67_{L U}$ & 0.864 & 0.642 & 0.524 & 0.616 & 0.469 & 0.459 \\
\hline$c P b 0.67_{05.95}$ & 0.879 & 0.809 & 0.792 & 0.823 & 0.779 & 0.805 \\
\hline$C c P b 0.67_{05.95}$ & 0.854 & 0.802 & 0.787 & 0.813 & 0.779 & 0.798 \\
\hline
\end{tabular}

Table B.17: SNCDs on Hill Model when $\alpha=0.01, \kappa=4, \eta=3$ and $k=6$ 


\begin{tabular}{|c|c|c|c|c|c|c|}
\hline \multirow[b]{2}{*}{$r=1000$} & \multicolumn{6}{|c|}{$n_{i}(\alpha=0.01, \kappa=5, \eta=1, k=4)$} \\
\hline & 50 & 100 & 200 & 300 & 400 & 500 \\
\hline$S N C D 1_{t r}$ & 1.31 & 0.86 & 0.58 & 0.48 & 0.40 & 0.36 \\
\hline$S \widehat{N C D} L 1_{n}$ & 1.00 & 0.72 & 0.51 & 0.42 & 0.36 & 0.32 \\
\hline$S \widehat{\widehat{N C D}} D 1_{n}$ & 1.39 & 0.90 & 0.60 & 0.48 & 0.41 & 0.36 \\
\hline$S N \widehat{\widehat{C D}} U 1_{n}$ & 2.35 & 1.19 & 0.72 & 0.55 & 0.46 & 0.40 \\
\hline Bias1.0 & 0.080 & 0.035 & 0.014 & 0.008 & 0.006 & 0.003 \\
\hline$c P b 1.0_{L U}$ & 0.829 & 0.706 & 0.705 & 0.693 & 0.636 & 0.652 \\
\hline$C c P b 1.0_{L U}$ & 0.850 & 0.713 & 0.712 & 0.702 & 0.633 & 0.659 \\
\hline$c P b 1.0_{05.95}$ & 0.931 & 0.908 & 0.909 & 0.924 & 0.899 & 0.917 \\
\hline$C c P b 1.0_{05.95}$ & 0.946 & 0.886 & 0.889 & 0.911 & 0.882 & 0.908 \\
\hline$S N C D 0.67_{t r}$ & 0.81 & 0.55 & 0.38 & 0.30 & 0.26 & 0.23 \\
\hline$\widehat{S N \widehat{C D L} 0.67_{n}}$ & 0.63 & 0.46 & 0.33 & 0.27 & 0.24 & 0.21 \\
\hline$S N \widehat{C D} 0.67_{n}$ & 0.87 & 0.58 & 0.39 & 0.31 & 0.27 & 0.24 \\
\hline$S N \widehat{C D U} 0.67_{n}$ & 1.35 & 0.77 & 0.47 & 0.36 & 0.30 & 0.26 \\
\hline Bias0.67 & 0.065 & 0.030 & 0.010 & 0.006 & 0.004 & 0.002 \\
\hline$c P b 0.67_{L U}$ & 0.746 & 0.667 & 0.684 & 0.677 & 0.628 & 0.639 \\
\hline$C c P b 0.67_{L U}$ & 0.749 & 0.656 & 0.687 & 0.675 & 0.625 & 0.641 \\
\hline$c P b 0.67_{05.95}$ & 0.955 & 0.913 & 0.921 & 0.930 & 0.905 & 0.920 \\
\hline$C c P b 0.67_{05.95}$ & 0.957 & 0.868 & 0.897 & 0.909 & 0.882 & 0.910 \\
\hline
\end{tabular}

Table B.18: SNCDs on Hill Model when $\alpha=0.01, \kappa=5, \eta=1$ and $k=4$ 


\begin{tabular}{|c|c|c|c|c|c|c|}
\hline \multirow[b]{2}{*}{$r=1000$} & \multicolumn{6}{|c|}{$n_{i}(\alpha=0.01, \kappa=5, \eta=1, k=6)$} \\
\hline & 50 & 100 & 200 & 300 & 400 & 500 \\
\hline $1_{t r}$ & 1.25 & 0.80 & 0.53 & 0.4 & 0.36 & 0.32 \\
\hline$\widehat{S N C D} L 1_{n}$ & 0.92 & 0.00 & 0.5 & 0.40 & 0.34 & 0.30 \\
\hline$\widehat{S \widehat{N C D}} D 1_{n}$ & 1.24 & 0.84 & 0.59 & 0.46 & 0.39 & 0.33 \\
\hline$S N \widehat{\overline{C D}} U 1_{n}$ & 2.20 & 1.09 & .72 & .54 & 44 & 0.38 \\
\hline Bias1.0 & .005 & 0.036 & 0.057 & 0.0 & 0.0 & 0.013 \\
\hline$c P b 1.0_{L}$ & 0.589 & 0. & 0.362 & 0.333 & 0.3 & 0.329 \\
\hline$C c P b 1.0_{L U}$ & 58 & 6 & 5 & 0 & 0.297 & 0.322 \\
\hline$c P b 1.0_{05 .}$ & 872 & 0. & 9 & 0.9 & .9 & 0.907 \\
\hline$C c P b 1.0_{05.95}$ & 0.868 & 0.775 & 0.833 & 0.852 & 0.874 & 0.883 \\
\hline$S N C D 0.67_{t r}$ & 0.15 & 0.50 & 0.34 & 0.28 & 0.24 & 0.21 \\
\hline$S N \widehat{C D L} 0.67_{n}$ & 0.64 & 0.46 & 0.3 & $0.2^{\prime}$ & 0.2 & 0.20 \\
\hline$S N \widehat{\overline{C D}} 0.67_{n}$ & 0.84 & 0.57 & 0.40 & 0.3 & J. & 0.22 \\
\hline$S N \widehat{C D U} 0.67_{n}$ & 1.23 & 0.74 & 0.4 & 0.3 & 0.2 & 0.25 \\
\hline Bias0.67 & 0.096 & $0.0^{\prime}$ & 0.0 & 0.0 & 0.0 & 0.011 \\
\hline$c P b 0.67_{L U}$ & 0.394 & 0.81 & 0.362 & 0.318 & 0.29 & 0.314 \\
\hline$C c P b 0.67_{L U}$ & 0.394 & 0.797 & 0.355 & 0.298 & 0.269 & 0.300 \\
\hline 067 & 0.808 & 0 . & 0. & 0.9 & 0.909 & 0.918 \\
\hline$C c P b 0.67_{05.95}$ & 0.810 & 0.797 & 0.839 & 0.852 & 0.879 & 0.886 \\
\hline
\end{tabular}

Table B.19: SNCDs on Hill Model when $\alpha=0.01, \kappa=5, \eta=1$ and $k=6$ 


\begin{tabular}{|c||c|c|c|c|c|c|}
\hline \multicolumn{1}{|c||}{} & \multicolumn{5}{c|}{$n_{i}(\alpha=0.01, \kappa=7, \eta=2, k=4)$} \\
\cline { 2 - 7 }$r=1000$ & 50 & 100 & 200 & 300 & 400 & 500 \\
\hline \hline$S N C D 1_{t r}$ & 3.33 & 2.42 & 1.75 & 1.44 & 1.26 & 1.13 \\
\hline$S N \widehat{\overline{C D}} L 1_{n}$ & 2.14 & 1.66 & 1.29 & 1.11 & 0.98 & 0.91 \\
$S \widehat{\widehat{N C D}} 1_{n}$ & 3.47 & 2.41 & 1.76 & 1.46 & 1.26 & 1.16 \\
$S N \widehat{\widehat{C D}} U 1_{n}$ & 4.62 & 3.27 & 2.33 & 1.91 & 1.62 & 1.48 \\
\hline Bias1.0 & 0.134 & -0.012 & 0.009 & 0.021 & 0.006 & 0.032 \\
\hline$c P b 1.0_{L U}$ & 0.959 & 0.953 & 0.889 & 0.842 & 0.812 & 0.818 \\
$C c P b 1.0_{L U}$ & 0.953 & 0.877 & 0.887 & 0.844 & 0.811 & 0.817 \\
\hline$c P b 1.0_{05.95}$ & 0.905 & 0.875 & 0.875 & 0.862 & 0.848 & 0.885 \\
$C c P b 1.0_{05.95}$ & 0.896 & 0.877 & 0.863 & 0.850 & 0.845 & 0.876 \\
\hline \hline$S N C D 0.67_{t r}$ & 2.30 & 1.66 & 1.19 & 0.98 & 0.85 & 0.77 \\
\hline$S N \widehat{\widehat{C D} 0.67_{n}}$ & 1.32 & 1.08 & 0.86 & 0.74 & 0.66 & 0.61 \\
$S N \widehat{\widehat{C D}} 0.67_{n}$ & 2.30 & 1.69 & 1.23 & 1.02 & 0.88 & 0.80 \\
$S N \widehat{\widehat{C D U}} 0.67_{n}$ & 3.56 & 2.50 & 1.76 & 1.42 & 1.19 & 1.08 \\
\hline$B i a s 0.67$ & 0.001 & 0.029 & 0.040 & 0.040 & 0.022 & 0.038 \\
\hline$c P b 0.67_{L U}$ & 0.950 & 0.907 & 0.839 & 0.842 & 0.812 & 0.785 \\
$C c P b 0.67_{L U}$ & 0.950 & 0.901 & 0.836 & 0.844 & 0.811 & 0.772 \\
\hline$c P b 0.67_{05.95}$ & 0.887 & 0.842 & 0.867 & 0.862 & 0.848 & 0.901 \\
$C c P b 0.67_{05.95}$ & 0.888 & 0.837 & 0.857 & 0.850 & 0.845 & 0.885 \\
\hline \hline
\end{tabular}

Table B.20: SNCDs on Hill Model when $\alpha=0.01, \kappa=7, \eta=2$ and $k=4$ 


\begin{tabular}{|c|c|c|c|c|c|c|}
\hline \multirow[b]{2}{*}{$r=1000$} & \multicolumn{6}{|c|}{$n_{i}(\alpha=0.01, \kappa=7, \eta=2, k=6)$} \\
\hline & 50 & 100 & 200 & 300 & 400 & 500 \\
\hline$S N C D 1_{t r}$ & 3.31 & 2.41 & 1.75 & 1.46 & 1.27 & 1.15 \\
\hline$S N \widehat{C D} L 1_{n}$ & 2.17 & 1.55 & 1.22 & 1.05 & 0.96 & 0.89 \\
\hline$S \widehat{N C D} 1_{n}$ & 3.41 & 2.40 & 1.66 & 1.38 & 1.25 & 1.14 \\
\hline$S N \widehat{C D} U 1_{n}$ & 4.67 & 3.21 & 2.24 & 1.82 & 1.60 & 1.45 \\
\hline Bias1.0 & 0.095 & -0.004 & -0.095 & -0.074 & -0.028 & -0.012 \\
\hline$c P b 1.0_{L U}$ & 0.975 & 0.963 & 0.815 & 0.685 & 0.649 & 0.599 \\
\hline$C c P b 1.0_{L U}$ & 0.968 & 0.963 & 0.758 & 0.648 & 0.634 & 0.597 \\
\hline$c P b 1.0_{05.95}$ & 0.922 & 0.925 & 0.854 & 0.828 & 0.821 & 0.831 \\
\hline$C c P b 1.0_{05.95}$ & 0.927 & 0.924 & 0.824 & 0.814 & 0.822 & 0.828 \\
\hline$S N C D 0.67_{t r}$ & 2.29 & 1.67 & 1.21 & 1.00 & 0.88 & 0.79 \\
\hline$S N \widehat{C D L} 0.67_{n}$ & 1.19 & 1.03 & 0.84 & 0.73 & 0.67 & 0.62 \\
\hline$S N \widehat{C D} 0.67_{n}$ & 2.28 & 1.59 & 1.18 & 01.00 & 0.90 & 0.82 \\
\hline$S N \widehat{C D U} 0.67_{n}$ & 3.50 & 2.41 & 1.66 & 1.36 & 1.21 & 1.09 \\
\hline Bias0.67 & -0.013 & -0.077 & -0.030 & -0.008 & 0.027 & 0.033 \\
\hline$c P b 0.67_{L U}$ & 0.975 & 0.832 & 0.598 & 0.531 & 0.536 & 0.506 \\
\hline$C c P b 0.67_{L U}$ & 0.968 & 0.801 & 0.592 & 0.532 & 0.541 & 0.507 \\
\hline$c P b 0.67_{05.95}$ & 0.922 & 0.842 & 0.776 & 0.789 & 0.810 & 0.829 \\
\hline$C c P b 0.67_{05.95}$ & 0.927 & 0.829 & 0.775 & 0.791 & 0.804 & 0.817 \\
\hline
\end{tabular}

Table B.21: SNCDs on Hill Model when $\alpha=0.01, \kappa=7, \eta=2$ and $k=6$ 


\begin{tabular}{|c|c|c|c|c|c|c|}
\hline \multirow[b]{2}{*}{$r=1000$} & \multicolumn{6}{|c|}{$n_{i}(\alpha=0.01, \kappa=8, \eta=5, k=4)$} \\
\hline & 50 & 100 & 200 & 300 & 400 & 500 \\
\hline$S N C D 1_{t r}$ & 5.99 & 5.26 & 4.64 & 4.32 & 4.10 & 3.95 \\
\hline$\widehat{S N C D} L 1_{n}$ & 5.09 & 4.45 & 3.61 & 3.10 & 2.80 & 2.70 \\
\hline$\widehat{S \widehat{N C D} D 1_{n}}$ & 5.97 & 5.28 & 4.67 & 4.32 & 4.12 & 3.96 \\
\hline$S N \widehat{\widehat{C D}} U 1_{n}$ & 6.81 & 5.78 & 5.03 & 4.68 & 4.50 & 4.60 \\
\hline Bias1.0 & -0.017 & 0.016 & 0.029 & 0.003 & 0.012 & 0.010 \\
\hline$c P b 1.0_{L U}$ & 0.675 & 0.644 & 0.698 & 0.799 & 0.902 & 0.917 \\
\hline$C c P b 1.0_{L U}$ & 0.675 & 0.637 & 0.681 & 0.796 & 0.888 & 0.913 \\
\hline$c P b 1.0_{05.95}$ & 0.771 & 0.796 & 0.861 & 0.888 & 0.936 & 0.930 \\
\hline$C c P b 1.0_{05.95}$ & 0.777 & 0.797 & 0.849 & 0.887 & 0.934 & 0.932 \\
\hline$S N C D 0.67_{t r}$ & 5.17 & 4.56 & 4.03 & 3.76 & 3.59 & 3.46 \\
\hline$S N \widehat{C D L} 0.67_{n}$ & 3.76 & 2.69 & 1.83 & 1.61 & 1.51 & 1.52 \\
\hline$S N \widehat{\widehat{C D}} 0.67_{n}$ & 5.24 & 4.61 & 4.02 & 3.70 & 3.52 & 3.41 \\
\hline$S N \widehat{C D U} 0.67_{n}$ & 6.16 & 5.28 & 4.63 & 4.69 & 4.17 & 4.05 \\
\hline Bias0.67 & 0.072 & 0.059 & -0.014 & -0.063 & -0.069 & -0.049 \\
\hline$c P b 0.67_{L U}$ & 0.758 & 0.799 & 0.919 & 0.941 & 0.955 & 0.944 \\
\hline$C c P b 0.67_{L U}$ & 0.745 & 0.787 & 0.927 & 0.947 & 0.968 & 0.952 \\
\hline$c P b 0.67_{05.95}$ & 0.796 & 0.847 & 0.916 & 0.919 & 0.916 & 0.886 \\
\hline$C c P b 0.67_{05.95}$ & 0.787 & 0.833 & 0.915 & 0.906 & 0.909 & 0.879 \\
\hline
\end{tabular}

Table B.22: SNCDs on Hill Model when $\alpha=0.01, \kappa=8, \eta=5$ and $k=4$ 


\begin{tabular}{|c|c|c|c|c|c|c|}
\hline \multirow[b]{2}{*}{$r=1000$} & \multicolumn{6}{|c|}{$n_{i}(\alpha=0.01, \kappa=8, \eta=5, k=6)$} \\
\hline & 50 & 100 & 200 & 300 & 400 & 500 \\
\hline$S N C D 1_{t r}$ & 5.93 & 5.22 & 4.61 & 4.30 & 4.10 & 3.95 \\
\hline$S \widehat{N C D} L 1_{n}$ & 4.93 & 4.33 & 3.73 & 3.34 & 3.12 & 2.95 \\
\hline$S \widehat{\overline{N C D}} D 1_{n}$ & 5.94 & 5.18 & 4.60 & 4.30 & 4.10 & 3.96 \\
\hline$S N \widehat{\widehat{C D}} U 1_{n}$ & 6.67 & 5.85 & 5.07 & 4.73 & 4.53 & 4.39 \\
\hline Bias1.0 & 0.013 & -0.038 & -0.013 & 0.003 & 0.008 & 0.006 \\
\hline$c P b 1.0_{L U}$ & 0.917 & 0.919 & 0.846 & 0.856 & 0.892 & 0.918 \\
\hline$C c P b 1.0_{L U}$ & 0.915 & 0.917 & 0.851 & 0.856 & 0.888 & 0.914 \\
\hline$c P b 1.0_{05.95}$ & 0.864 & 0.869 & 0.833 & 0.839 & 0.883 & 0.904 \\
\hline$C c P b 1.0_{05.95}$ & 0.862 & 0.869 & 0.837 & 0.838 & 0.879 & 0.904 \\
\hline$S N C D 0.67_{t r}$ & 5.12 & 4.52 & 4.03 & 3.78 & 3.62 & 3.50 \\
\hline$S N \widehat{C D L} 0.67_{n}$ & 3.78 & 2.83 & 1.89 & 1.43 & 1.34 & 1.33 \\
\hline$S N \widehat{\overline{C D}} 0.67_{n}$ & 5.10 & 4.52 & 4.03 & 3.73 & 3.57 & 3.42 \\
\hline$S N \widehat{C D U} 0.67_{n}$ & 6.01 & 5.22 & 4.63 & 4.37 & 4.18 & 4.05 \\
\hline Bias0.67 & -0.017 & -0.009 & 0.000 & -0.056 & -0.053 & -0.081 \\
\hline$c P b 0.67_{L U}$ & 0.865 & 0.871 & 0.927 & 0.932 & 0.946 & 0.935 \\
\hline$C c P b 0.67_{L U}$ & 0.865 & 0.875 & 0.927 & 0.945 & 0.953 & 0.945 \\
\hline$c P b 0.67_{05.95}$ & 0.808 & 0.812 & 0.884 & 0.899 & 0.917 & 0.901 \\
\hline$C c P b 0.67_{05.95}$ & 0.796 & 0.813 & 0.884 & 0.897 & 0.909 & 0.885 \\
\hline
\end{tabular}

Table B.23: SNCDs on Hill Model when $\alpha=0.01, \kappa=8, \eta=5$ and $k=6$

\section{B.2.2 Non-parametric Bootstrap Method Results}

Tables summarize the results of SNCD estimates all possible combination of selected Hill model parameter combination, numbers of dose and sizes per dose when using parametric bootstrap method. These results are shown in Figures 3.8, 3.9 and 3.10 


\begin{tabular}{|c|c|c|c|c|c|c|}
\hline \multirow[b]{2}{*}{$r=1000$} & \multicolumn{6}{|c|}{$n_{i}(\alpha=0.01, \kappa=4, \eta=3, k=4)$} \\
\hline & 50 & 100 & 200 & 300 & 400 & 500 \\
\hline$S N C D 1_{t r}$ & 2.01 & & & 0.00 & & 1.09 \\
\hline$\widehat{S N C D} L 1_{n}$ & 1.85 & 1.50 & 1.19 & 0.84 & 1.01 & 0.92 \\
\hline$\widehat{S \widehat{N C D}} 1_{n}$ & 2.31 & 1.84 & 1.41 & 0.99 & 1.20 & 1.08 \\
\hline$S N \widehat{\widehat{C D}} U 1_{n}$ & 2.90 & 2.23 & 1.66 & 1.18 & 1.42 & 1.27 \\
\hline Bias1.0 & 00 & 0.0 & 0.003 & .007 & .004 & -0.005 \\
\hline$c P b 1$ & 0065 & 0.868 & 0798 & 0.708 & 0.790 & 735 \\
\hline$C c P b 1.0_{L U}$ & oct & م & ( 700 & 0705 & 0.790 & 0.730 \\
\hline$c P b 1.0_{05.95}$ & 922 & 0.8 & 0.885 & 0.880 & 0.882 & 0.863 \\
\hline$C c P b 1.0_{05}$ & 0.921 & 087 & 0.881 & 0.872 & 0.884 & 0.865 \\
\hline$S N C D 0.67_{t r}$ & 1.7 & & 1.03 & 0.013 & 0.87 & 0.79 \\
\hline$S N \widehat{C D L} 0.67_{n}$ & 1 . & 1 & 0.85 & 0 & 0 & 0.66 \\
\hline$S N \widehat{\widehat{C D}} 0.67_{n}$ & 1.78 & 1.38 & 1.04 & 0.70 & 0.88 & 0.79 \\
\hline$S N \widehat{C D U} 0.67_{n}$ & 2.33 & 1 & 1.29 & 0.86 & 1.09 & 0.97 \\
\hline Bias0.67 & $0.00 x$ & 0.014 & 0.012 & 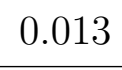 & 0.012 & 0.003 \\
\hline$c P b 0.67_{L U}$ & 0.892 & 0.779 & 0.733 & 0.665 & 0.742 & 0.693 \\
\hline$C c P b 0.67_{L U}$ & 0 & 0.7 & 07 & 0.665 & 36 & 0.693 \\
\hline$c P b 0.67_{05.95}$ & 0 & . & $0 . c^{2}$ & 0.878 & 0.881 & 0.84 \\
\hline$C c P b 0.67_{05.95}$ & 0.882 & 0.848 & 0.868 & 0.869 & 0.874 & 0.847 \\
\hline
\end{tabular}

Table B.24: SNCDs on Hill Model when $\alpha=0.01, \kappa=4, \eta=3$ and $k=4$ 


\begin{tabular}{|c|c|c|c|c|c|c|}
\hline \multirow[b]{2}{*}{$r=1000$} & \multicolumn{6}{|c|}{$n_{i}(\alpha=0.01, \kappa=4, \eta=3, k=6)$} \\
\hline & 50 & 100 & 200 & 300 & 400 & 500 \\
\hline$S N C D 1_{t r}$ & 2.42 & 1.94 & 1.64 & 1.54 & 1.40 & 1.43 \\
\hline$S N \widehat{C D} L 1_{n}$ & 1.65 & 1.69 & 1.32 & 1.21 & 1.12 & 1.16 \\
\hline$S \widehat{N C D} 1_{n}$ & 2.42 & 1.92 & 1.5 & 1.48 & 1.36 & 1.39 \\
\hline$S N \widehat{C D} U 1_{n}$ & 3.31 & 2.18 & 1.83 & 1.94 & 1.61 & 1.62 \\
\hline Bias1.0 & 0.002 & -0.019 & -0.074 & -0.060 & -0.041 & -0.044 \\
\hline$c P b 1.0_{L U}$ & 0.972 & 0.916 & 0.678 & 0.700 & 0.647 & 0.661 \\
\hline$C c P b 1.0_{L U}$ & 0.972 & 0.903 & 0.636 & 0.682 & 0.621 & 0.626 \\
\hline$c P b 1.0_{05.95}$ & 0.947 & 0.947 & 0.874 & 0.858 & 0.835 & 0.870 \\
\hline$C c P b 1.0_{05.95}$ & 0.947 & 0.925 & 0.840 & 0.833 & 0.817 & 0.864 \\
\hline$S N C D 0.67_{t r}$ & 1.87 & 1.71 & 1.37 & 1.24 & 1.12 & 1.18 \\
\hline$S N \widehat{C D L} 0.67_{n}$ & 1.17 & 1.45 & 1.05 & 0.93 & 0.86 & 0.91 \\
\hline$S N \widehat{C D} 0.67_{n}$ & 1.75 & 1.67 & 1.28 & 1.19 & 1.09 & 1.14 \\
\hline$S N \widehat{C D U} 0.67_{n}$ & 2.53 & 1.92 & 1.54 & 1.48 & 1.35 & 1.39 \\
\hline Tr.Bias0.67 & -0.115 & -0.041 & -0.088 & -0.054 & -0.031 & -0.043 \\
\hline$c P b 0.67_{L U}$ & 0.916 & 0.765 & 0.549 & 0.595 & 0.549 & 0.563 \\
\hline$C c P b 0.67_{L U}$ & 0.872 & 0.720 & 0.512 & 0.578 & 0.550 & 0.539 \\
\hline$c P b 0.67_{05.95}$ & 0.897 & 0.905 & 0.825 & 0.810 & 0.802 & 0.826 \\
\hline$C c P b 0.67_{05.95}$ & 0.871 & 0.877 & 0.803 & 0.797 & 0.786 & 0.823 \\
\hline
\end{tabular}

Table B.25: SNCDs on Hill Model when $\alpha=0.01, \kappa=4, \eta=3$ and $k=6$ 


\begin{tabular}{|c|c|c|c|c|c|c|}
\hline \multirow[b]{2}{*}{$r=1000$} & \multicolumn{6}{|c|}{$n_{i}(\alpha=0.01, \kappa=5, \eta=1, k=4)$} \\
\hline & 50 & 100 & 200 & 300 & 400 & 500 \\
\hline$S N C D 1_{t r}$ & 1.35 & 0.99 & 0.53 & 0.51 & 0.44 & 0.38 \\
\hline$S N \widehat{C D} L 1_{n}$ & 1.04 & 0.82 & 0.47 & 0.45 & 0.40 & 0.35 \\
\hline$\widehat{S N C D} 1_{n}$ & 1.54 & 1.03 & 0.55 & 0.51 & 0.44 & 0.39 \\
\hline$S N \widehat{C D} U 1_{n}$ & 2.92 & 1.36 & 0.66 & 0.60 & 0.50 & 0.43 \\
\hline Bias1.0 & 0.197 & 0.036 & 0.011 & 0.008 & 0.007 & 0.003 \\
\hline$c P b 1.0_{L U}$ & 0.820 & 0.714 & 0.713 & 0.637 & 0.640 & 0.637 \\
\hline$C c P b 1.0_{L U}$ & 0.870 & 0.717 & 0.710 & 0.638 & 0.653 & 0.643 \\
\hline$c P b 1.0_{05.95}$ & 0.902 & 0.886 & 0.913 & 0.901 & 0.914 & 0.897 \\
\hline$C c P b 1.0_{05.95}$ & 0.947 & 0.874 & 0.893 & 0.879 & 0.895 & 0.895 \\
\hline$S N C D 0.67_{t r}$ & 0.81 & 0.64 & 0.34 & 0.006 & 0.29 & 0.002 \\
\hline$S N \widehat{C D L} 0.67_{n}$ & 0.64 & 0.54 & 0.30 & 0.30 & 0.26 & 0.23 \\
\hline$S N \widehat{C D} 0.67_{n}$ & 0.87 & 0.68 & 0.3 & 0.34 & 0.29 & 0.25 \\
\hline$S N \widehat{C D U} 0.67_{n}$ & 1.41 & 0.90 & 0.42 & 0.39 & 0.33 & 0.28 \\
\hline Bias0.67 & 0.066 & 0.037 & 0.007 & 0.33 & 0.005 & 0.002 \\
\hline$c P b 0.67_{L U}$ & 0.732 & 0.669 & 0.697 & 0.613 & 0.629 & 0.620 \\
\hline$C c P b 0.67_{L U}$ & 0.730 & 0.664 & 0.693 & 0.606 & 0.634 & 0.628 \\
\hline$c P b 0.67_{05.95}$ & 0.944 & 0.899 & 0.917 & 0.901 & 0.917 & 0.905 \\
\hline$C c P b 0.67_{05.95}$ & 0.963 & 0.876 & 0.890 & 0.883 & 0.898 & 0.896 \\
\hline
\end{tabular}

Table B.26: SNCDs on Hill Model when $\alpha=0.01, \kappa=5, \eta=1$ and $k=4$ 


\begin{tabular}{|c|c|c|c|c|c|c|}
\hline \multirow[b]{2}{*}{$r=1000$} & \multicolumn{6}{|c|}{$n_{i}(\alpha=0.01, \kappa=5, \eta=1, k=6)$} \\
\hline & 50 & 100 & 200 & 300 & 400 & 500 \\
\hline$D 1_{t r}$ & 1.44 & 0.0 & 0.76 & 0.40 & 0.31 & 0.29 \\
\hline$\widehat{S N C D} L 1_{n}$ & 1.11 & 0.00 & 0.46 & 0.38 & 0.30 & 0.27 \\
\hline$\widehat{S \widehat{N C D}} D 1_{n}$ & 1.38 & 0.94 & 0.53 & 0.43 & 0.33 & 0.30 \\
\hline$S N \widehat{\overline{C D}} U 1_{n}$ & 2.28 & 1.15 & 63 & .50 & 37 & 0.34 \\
\hline Bias1.0 & .060 & 0.035 & 0.052 & 0.0 & 0.0 & 0.019 \\
\hline$c P b 1.0_{L}$ & 0.545 & 0. & 0.324 & 0.314 & 0.3 & 0.300 \\
\hline$C c P b 1.0_{L U}$ & 0.512 & 2 & 9 & 0 . & 0 & 0.278 \\
\hline$c P b 1.0_{05 .}$ & 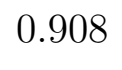 & 0.7 & 0.861 & 0.8 & .8 & 0.929 \\
\hline$C c P b 1.0_{05.95}$ & 0.915 & 0.766 & 0.828 & 0.863 & 0.875 & 0.884 \\
\hline$S N C D 0.67_{t r}$ & 1.01 & 0.00 & 0.30 & 0.26 & 0.20 & 0.18 \\
\hline$\widehat{S N \widehat{C D L} 0.67_{n}}$ & 0.83 & 0.5 & 0.30 & 0.25 & 0.19 & 0.18 \\
\hline$S N \widehat{\overline{C D}} 0.67_{n}$ & 1.00 & 0.68 & 0 & 0.2 & 0.21 & 0.20 \\
\hline$S N \widehat{C D U} 0.67_{n}$ & 1.67 & 0.8 & 0.4 & 0.3 & 0.24 & 0.22 \\
\hline Bias0.67 & -0.013 & 0.0 & 0.0 & 0.0 & .0 & 0.014 \\
\hline$c P b 0.67_{L U}$ & 0.338 & 0.278 & 0.302 & 0.295 & 0.34 & 0.289 \\
\hline$C c P b 0.67_{L U}$ & 0.332 & 0.259 & 0.285 & 0.297 & 0.328 & 0.270 \\
\hline$c P b 0.67_{05.95}$ & 0.776 & 0 & 0 . & 0.9 & 0.899 & 0.936 \\
\hline$C c P b 0.67_{05.95}$ & 0.782 & 0.770 & 0.837 & 0.865 & 0.875 & 0.886 \\
\hline
\end{tabular}

Table B.27: SNCDs on Hill Model when $\alpha=0.01, \kappa=5, \eta=1$ and $k=6$ 


\begin{tabular}{|c|c|c|c|c|c|c|}
\hline \multirow[b]{2}{*}{$r=1000$} & \multicolumn{6}{|c|}{$n_{i}(\alpha=0.01, \kappa=7, \eta=2, k=4)$} \\
\hline & 50 & 100 & 200 & 300 & 400 & 500 \\
\hline$D 1_{t r}$ & 3.52 & 3.43 & 1.61 & 1.15 & 1.08 & 1.05 \\
\hline$S N \widehat{C D} L 1_{n}$ & 2.51 & 2.14 & 1.22 & 0.92 & 0.87 & 0.53 \\
\hline$\widehat{S \widehat{N C D}} D 1_{n}$ & 3.66 & 3.45 & 1.62 & 1.19 & 1.11 & 0.71 \\
\hline$S N \widehat{\overline{C D}} U 1_{n}$ & 4.72 & 4.21 & 41 & 1.57 & .46 & 0.99 \\
\hline Bias1. & 0.142 & 0.017 & 0.008 & 0.037 & 0.0 & 0.014 \\
\hline$c P b 1.0_{L}$ & 954 & 0.949 & 0.7 & 0.804 & 0.8 & 0.818 \\
\hline$C c P b 1.0_{L U}$ & 551 & 0.949 & 0 & 0.799 & 0 & 0.819 \\
\hline$c P b 1.0_{05 .}$ & 0.929 & 0.872 & 0.859 & 0.8 & .8 & 0.873 \\
\hline$C c P b 1.0_{05.95}$ & 0.909 & 0.869 & 0.852 & 0.871 & 0.886 & 0.865 \\
\hline$S N C D 0.67_{t r}$ & .44 & 2 & 1.06 & 0.1 & 0.70 & 0.69 \\
\hline$\widehat{S N \widehat{C D L} 0.67_{n}}$ & 1.60 & 1.35 & 0.8 & 0.60 & 0.56 & 0.53 \\
\hline$S N \widehat{\overline{C D}} 0.67_{n}$ & 2.4 & 2.62 & 1 & 0 . & 0.73 & 0.71 \\
\hline$S N \widehat{\overline{C D U}} 0.67_{n}$ & 3.65 & 3.60 & 1.5 & 1.0 & 0.99 & 0.99 \\
\hline Bias0.67 & 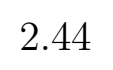 & -0.054 & 0.0 & 0.0 & 0.0 & 0.021 \\
\hline$c P b 0.67_{L U}$ & 0.944 & 0.918 & 0.781 & 0.769 & 0.791 & 0.791 \\
\hline$C c P b 0.67_{L U}$ & 0.942 & 0.930 & 0.789 & 0.757 & 0.771 & 0.784 \\
\hline$c P b 0.67_{05.95}$ & 0 & 6 & 0.860 & 0.904 & 0.906 & 0.882 \\
\hline$C c P b 0.67_{05.95}$ & 0.922 & 0.812 & 0.846 & 0.877 & 0.885 & 0.785 \\
\hline
\end{tabular}

Table B.28: SNCDs on Hill Model when $\alpha=0.01, \kappa=7, \eta=2$ and $k=4$ 


\begin{tabular}{|c||c|c|c|c|c|c|}
\hline \multicolumn{1}{|c||}{} & \multicolumn{5}{c|}{$n_{i}(\alpha=0.01, \kappa=7, \eta=2, k=6)$} \\
\cline { 2 - 7 }$r=1000$ & 50 & 100 & 200 & 300 & 400 & 500 \\
\hline \hline$S N C D 1_{t r}$ & 2.85 & 2.63 & 1.62 & 1.90 & 1.17 & 0.86 \\
\hline$S N \widehat{\overline{C D}} L 1_{n}$ & 2.11 & 1.43 & 1.04 & 1.35 & 0.90 & 0.73 \\
$S \widehat{\widehat{N C D}} 1_{n}$ & 2.85 & 2.22 & 1.55 & 1.84 & 1.17 & 0.89 \\
$S N \widehat{\widehat{C D}} U 1_{n}$ & 3.46 & 2.89 & 2.33 & 2.33 & 1.53 & 1.10 \\
\hline Bias 1.0 & 0.002 & 0.087 & -0.071 & -0.060 & -0.006 & 0.028 \\
\hline$c P b 1.0_{L U}$ & 0.902 & 0.949 & 0.796 & 0.903 & 0.595 & 0.479 \\
$C c P b 1_{L U}$ & 0.903 & 0.923 & 0.769 & 0.900 & 0.595 & 0.479 \\
\hline$c P b 1.0_{05.95}$ & 0.898 & 0.914 & 0.835 & 0.892 & 0.808 & 0.816 \\
$C c P b 1.0_{05.95}$ & 0.899 & 0.909 & 0.811 & 0.876 & 0.808 & 0.807 \\
\hline \hline$S N C D 0.67_{t r}$ & 2.10 & 2.23 & 0.92 & 1.46 & 0.77 & 0.56 \\
\hline$S N \widehat{\overline{C D} 0.67_{n}}$ & 1.28 & 1.43 & 0.67 & 0.98 & 0.61 & 0.49 \\
$S N \widehat{\widehat{C D}} 0.67_{n}$ & 2.01 & 2.20 & 1.00 & 1.41 & 0.83 & 0.62 \\
$S N \widehat{\widehat{C D U}} 0.67_{n}$ & 2.83 & 2.89 & 1.55 & 1.91 & 1.13 & 1.01 \\
\hline Bias0.67 & -0.086 & -0.032 & 0.083 & -0.057 & 0.055 & 0.055 \\
\hline$c P b 0.67_{L U}$ & 0.942 & 0.941 & 0.565 & 0.766 & 0.483 & 0.408 \\
$C c P b 0.67_{L U}$ & 0.904 & 0.950 & 0.576 & 0.742 & 0.505 & 0.413 \\
\hline$c P b 0.67_{05.95}$ & 0.869 & 0.944 & 0.796 & 0.827 & 0.813 & 0.840 \\
$C c P b 0.67_{05.95}$ & 0.849 & 0.944 & 0.796 & 0.816 & 0.809 & 0.814 \\
\hline \hline
\end{tabular}

Table B.29: SNCDs on Hill Model when $\alpha=0.01, \kappa=7, \eta=2$ and $k=6$ 


\begin{tabular}{|c|c|c|c|c|c|c|}
\hline \multirow[b]{2}{*}{$r=1000$} & \multicolumn{6}{|c|}{$n_{i}(\alpha=0.01, \kappa=8, \eta=5, k=4)$} \\
\hline & 50 & 100 & 200 & 300 & 400 & 500 \\
\hline$S N C D 1_{t r}$ & 6.03 & 5.23 & 4.52 & 5.04 & 4.62 & 4.21 \\
\hline$S \widehat{\widehat{C D D}} L 1_{n}$ & 4.91 & 4.27 & 4.05 & 4.15 & 3.81 & 3.08 \\
\hline$\widehat{S \widehat{N C D}} 1_{n}$ & 5.92 & 5.25 & 4.57 & 5.07 & 4.63 & 4.21 \\
\hline$S \widehat{\overline{C D}} U 1_{n}$ & 6.51 & 5.88 & 4.75 & 5.38 & 4.87 & 4.54 \\
\hline Bias 1.0 & -0.103 & 0.018 & 0.041 & 0.026 & 0.0004 & -0.005 \\
\hline$c P b 1.0_{L U}$ & 0.599 & 0.657 & 0.623 & 0.585 & 0.569 & 0.828 \\
\hline$C c P b 1.0_{L U}$ & 0.605 & 0.653 & 0.572 & 0.573 & 0.569 & 0.832 \\
\hline$c P b 1.0_{05.95}$ & 0.758 & 0.780 & 0.897 & 0.827 & 0.880 & 0.920 \\
\hline$C c P b 1.0_{05.95}$ & 0.766 & 0.784 & 0.866 & 0.820 & 0.880 & 0.923 \\
\hline$S N C D 0.67_{t r}$ & 5.44 & 3.87 & 4.28 & 4.58 & 4.27 & 3.80 \\
\hline 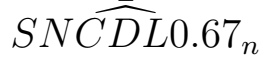 & 2.64 & 2.17 & 3.24 & 1.91 & 2.23 & 1.70 \\
\hline$S N \widehat{\overline{C D}} 0.67_{n}$ & 5.38 & 4.05 & 4.30 & 4.60 & 4.25 & 3.75 \\
\hline$S N \widehat{\widehat{C D U}} 0.67_{n}$ & 6.10 & 5.10 & 4.57 & 5.09 & 4.64 & 4.27 \\
\hline Bias0.67 & -0.066 & 0.185 & 0.018 & 0.024 & -0.021 & -0.050 \\
\hline$c P b 0.67_{L U}$ & 0.599 & 0.917 & 0.824 & 0.748 & 0.814 & 0.936 \\
\hline$C c P b 0.67_{L U}$ & 0.605 & 0.880 & 0.810 & 0.736 & 0.829 & 0.956 \\
\hline$c P b 0.67_{05.95}$ & 0.758 & 0.837 & 0.949 & 0.878 & 0.941 & 0.949 \\
\hline$C c P b 0.67_{05.95}$ & 0.766 & 0.830 & 0.942 & 0.868 & 0.944 & 0.942 \\
\hline
\end{tabular}

Table B.30: SNCDs on Hill Model when $\alpha=0.01, \kappa=8, \eta=5$ and $k=4$ 


\begin{tabular}{|c|c|c|c|c|c|c|}
\hline \multirow[b]{2}{*}{$r=1000$} & \multicolumn{6}{|c|}{$n_{i}(\alpha=0.01, \kappa=8, \eta=5, k=6)$} \\
\hline & 50 & 100 & 200 & 300 & 400 & 500 \\
\hline$S N C D 1_{t r}$ & 5.90 & 4.41 & 4.11 & 4.39 & 4.49 & 3.68 \\
\hline$S N \widehat{C D} L 1_{n}$ & 4.97 & 4.00 & 3.77 & 3.63 & 3.21 & 2.56 \\
\hline$S \widehat{N C D} 1_{n}$ & 5.95 & 4.46 & 4.17 & 4.38 & 4.45 & 3.68 \\
\hline$S N \widehat{C D} U 1_{n}$ & 6.55 & 5.07 & 4.66 & 4.73 & 4.89 & 4.05 \\
\hline Bias1.0 & 0.045 & 0.052 & 0.058 & -0.016 & -0.038 & 0.001 \\
\hline$c P b 1.0_{L U}$ & 0.943 & 0.930 & 0.892 & 0.849 & 0.960 & 0.938 \\
\hline$C c P b 1.0_{L U}$ & 0.936 & 0.904 & 0.885 & 0.854 & 0.876 & 0.938 \\
\hline$c P b 1.0_{05.95}$ & 0.885 & 0.927 & 0.857 & 0.847 & 0.846 & 0.944 \\
\hline$C c P b 1.0_{05.95}$ & 0.878 & 0.916 & 0.846 & 0.855 & 0.851 & 0.943 \\
\hline$S N C D 0.67_{t r}$ & 5.29 & 4.12 & 3.64 & 3.98 & 3.90 & 3.35 \\
\hline$S N \widetilde{C D L} 0.67_{n}$ & 4.36 & 3.72 & 3.14 & 1.43 & 1.54 & 0.69 \\
\hline$S N \widehat{C D} 0.67_{n}$ & 5.30 & 4.15 & 3.69 & 3.93 & 3.80 & 3.08 \\
\hline$S N \widehat{C D U} 0.67_{n}$ & 6.05 & 4.55 & 4.09 & 4.42 & 4.54 & 3.75 \\
\hline Bias0.67 & 0.010 & 0.031 & 0.047 & -0.050 & -0.104 & -0.271 \\
\hline$c P b 0.67_{L U}$ & 0.940 & 0.934 & 0.828 & 0.931 & 0.927 & 0.924 \\
\hline$C c P b 0.67_{L U}$ & 0.943 & 0.902 & 0.894 & 0.947 & 0.953 & 0.964 \\
\hline$c P b 0.67_{05.95}$ & 0.848 & 0.919 & 0.943 & 0.923 & 0.869 & 0.846 \\
\hline$C c P b 0.67_{05.95}$ & 0.850 & 0.905 & 0.942 & 0.921 & 0.862 & 0.777 \\
\hline
\end{tabular}

Table B.31: SNCDs on Hill Model when $\alpha=0.01, \kappa=8, \eta=5$ and $k=6$ 


\section{APPENDIX OF CHAPTER 4}

\section{C.1 Histogram of Ratio of BMD and SNCD based on Simulation Results}

Histograms showing the distribution of BMD to SNCD ratio estimated for four selected Hill model parameter combination with varying sizes (50, 200, and 500) per dose and numbers (4 and 6) of dose. Results are discussed in Chapter 4.4.3.1.
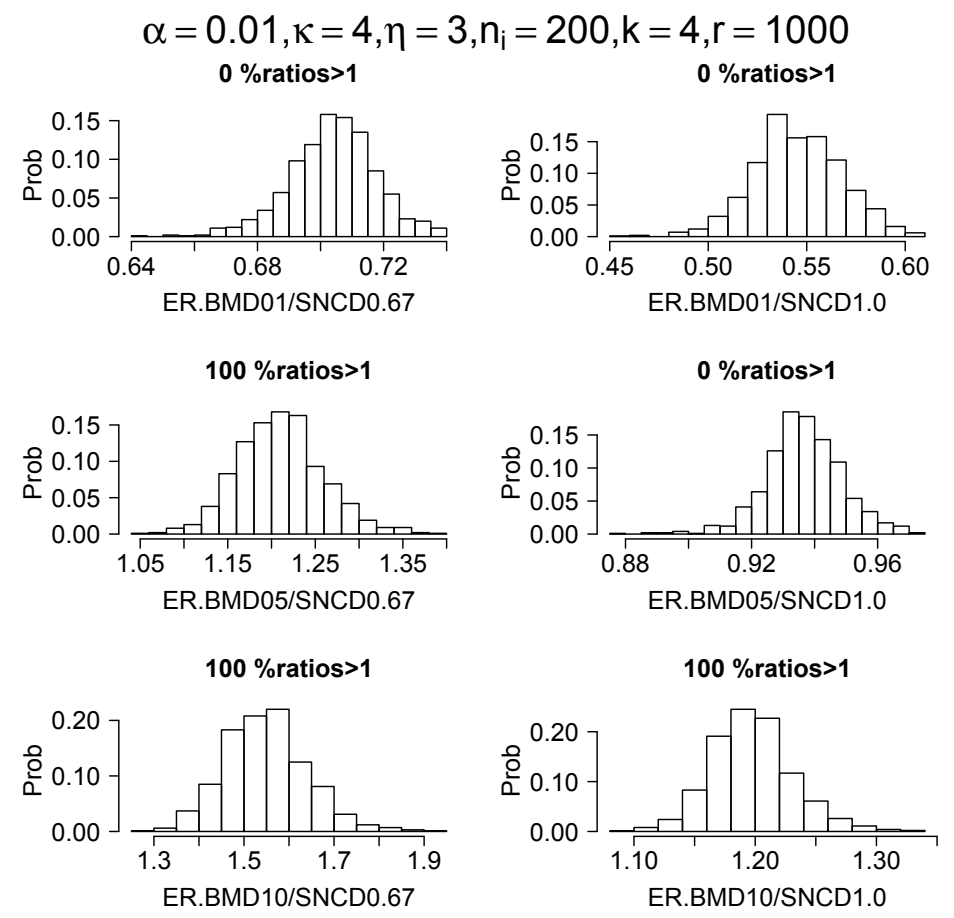

Figure C.1: Histogram for BMD/SNCD when $\kappa=4, \eta=3, k=4, n_{i}=200$ 


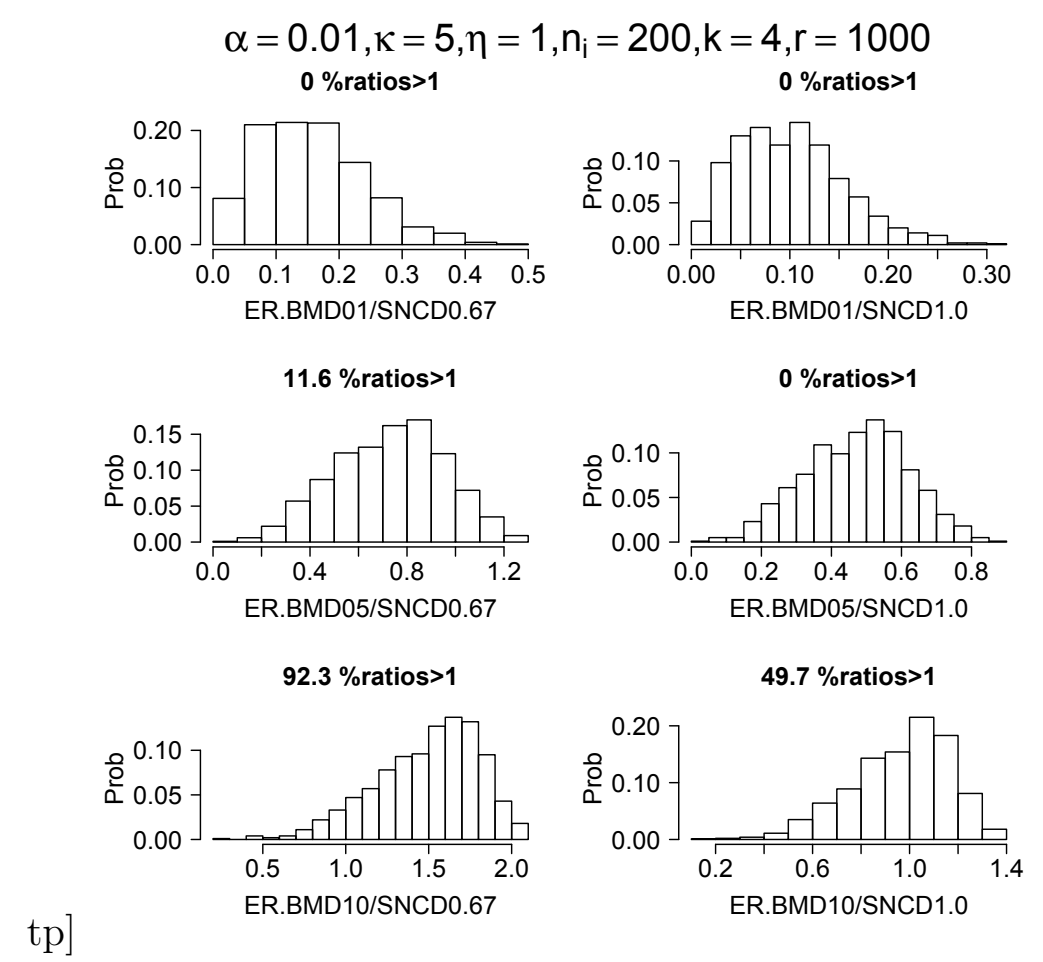

Figure C.2: Histogram for BMD/SNCD when $\kappa=5, \eta=1, k=4, n_{i}=200$

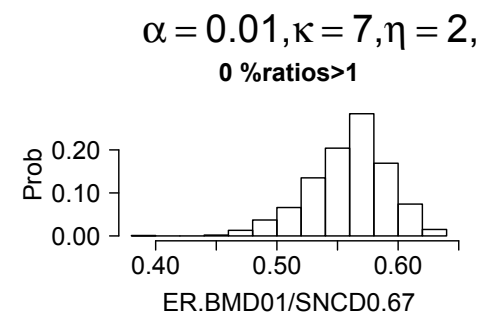

$$
\begin{array}{r}
n_{i}=200, k=4, r=1000 \\
0 \% \text { ratios }>1
\end{array}
$$


Figure C.3: Histogram for BMD/SNCD when $\kappa=7, \eta=2, k=4, n_{i}=200$ 

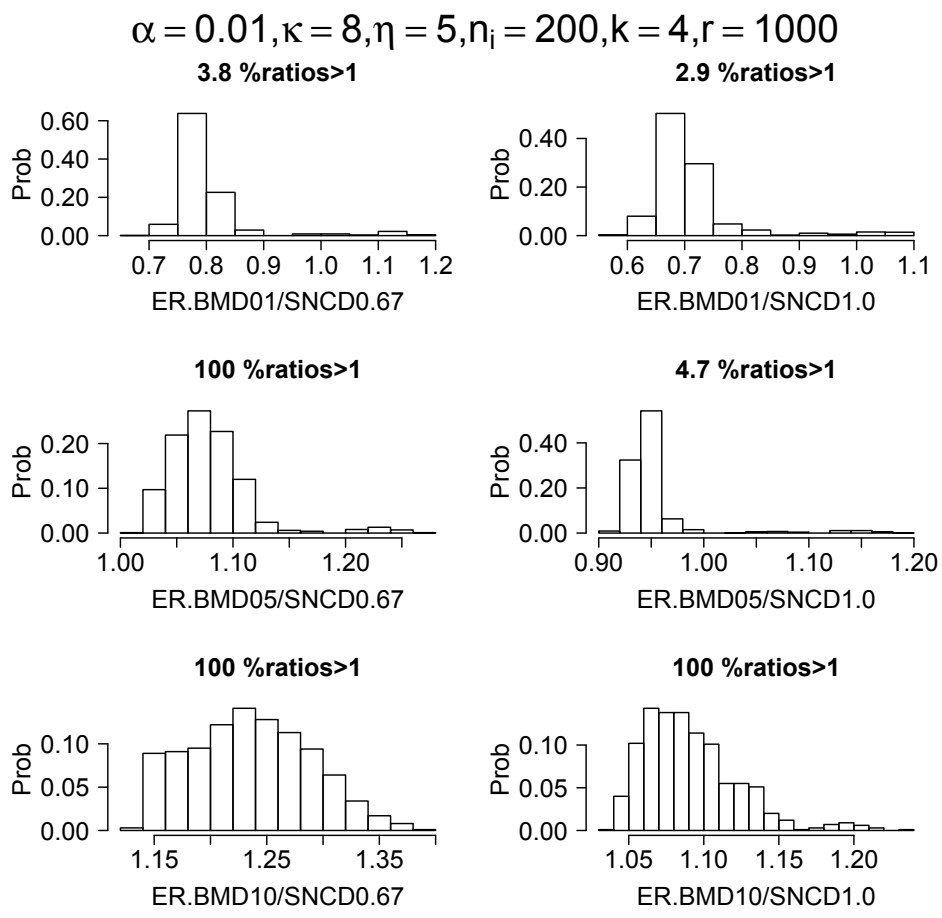

Figure C.4: Histogram for BMD/SNCD when $\kappa=8, \eta=5, k=4, n_{i}=200$

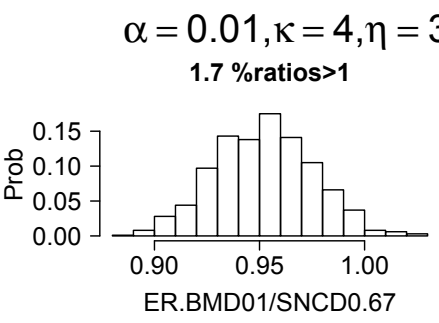

$$
\begin{array}{r}
n_{i}=500, k=4, r=1000 \\
0 \% \text { ratios }>1
\end{array}
$$


Figure C.5: Histogram for BMD/SNCD when $\kappa=4, \eta=3, k=4, n_{i}=500$ 

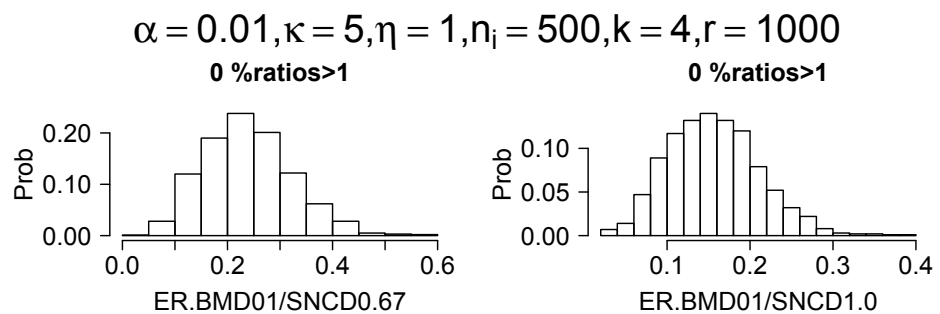

$77.1 \%$ ratios $>1$

$6.4 \%$ ratios $>1$
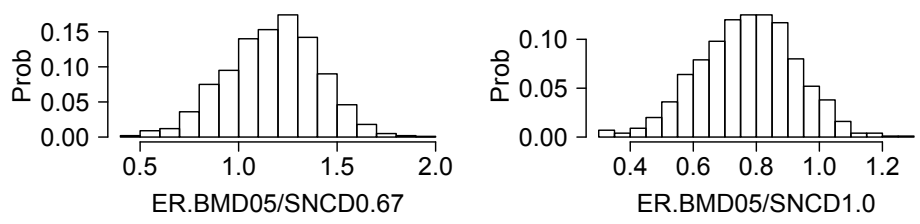

$100 \%$ ratios $>1$

$99.2 \%$ ratios $>1$
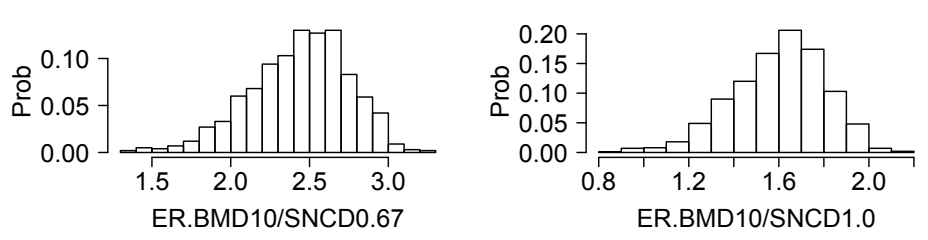

Figure C.6: Histogram for BMD/SNCD when $\kappa=5, \eta=1, k=4, n_{i}=500$


Figure C.7: Histogram for BMD/SNCD when $\kappa=7, \eta=2, k=4, n_{i}=500$ 

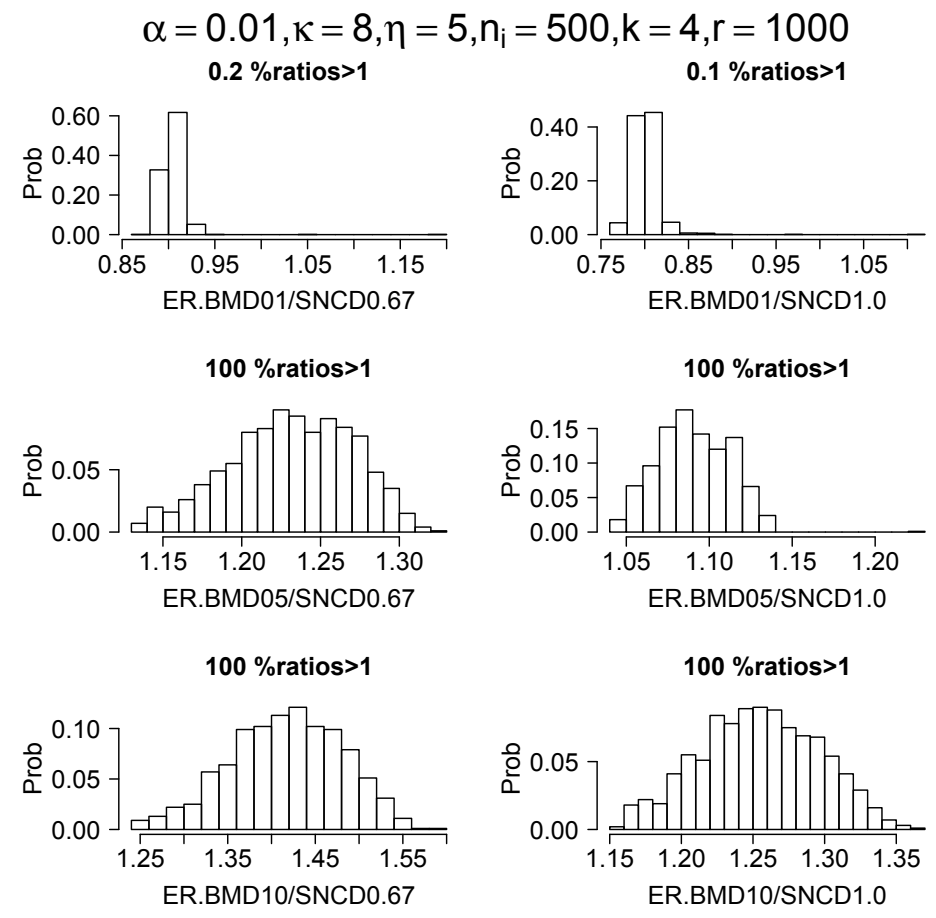

Figure C.8: Histogram for BMD/SNCD when $\kappa=8, \eta=5, k=4, n_{i}=500$
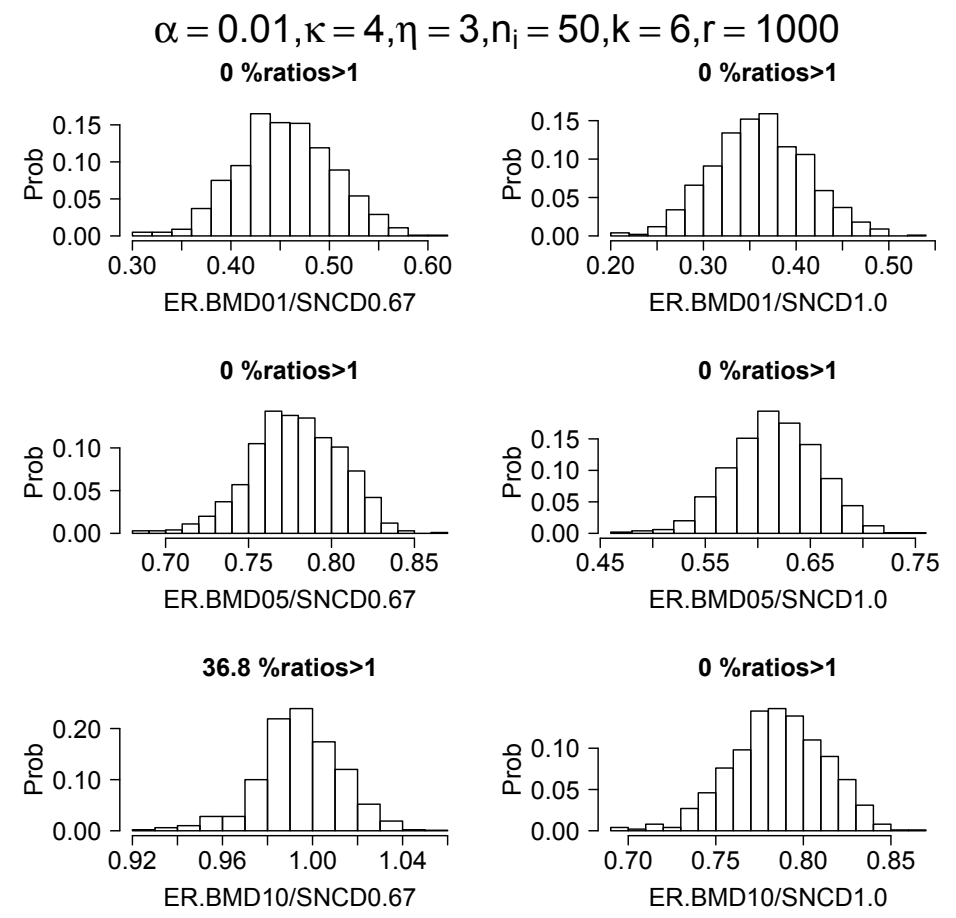

Figure C.9: Histogram for BMD/SNCD when $\kappa=4, \eta=3, k=6, n_{i}=50$ 


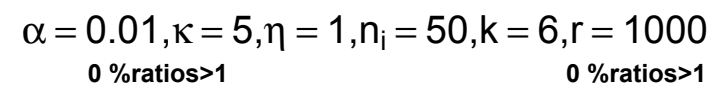

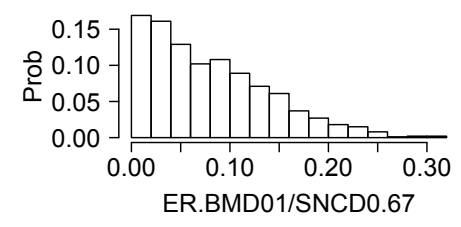

$0 \%$ ratios $>1$

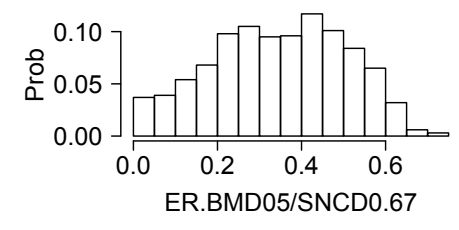

$3.2 \%$ ratios $>1$

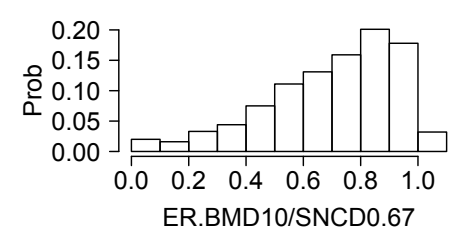

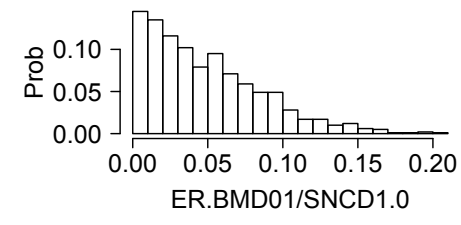

$0 \%$ ratios $>1$

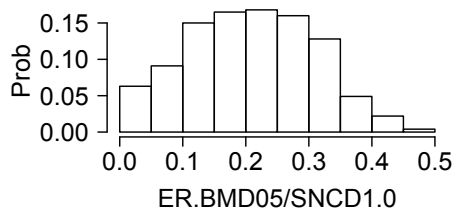

$0 \%$ ratios $>1$



Figure C.10: Histogram for BMD/SNCD when $\kappa=5, \eta=1, k=6, n_{i}=50$

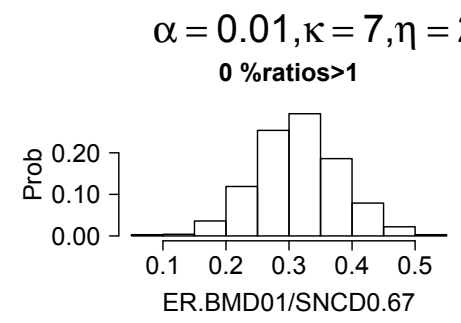

$2, n_{i}=50, k=6, r=1000$
$0 \%$ ratios $>1$

$0 \%$ ratios $>1$
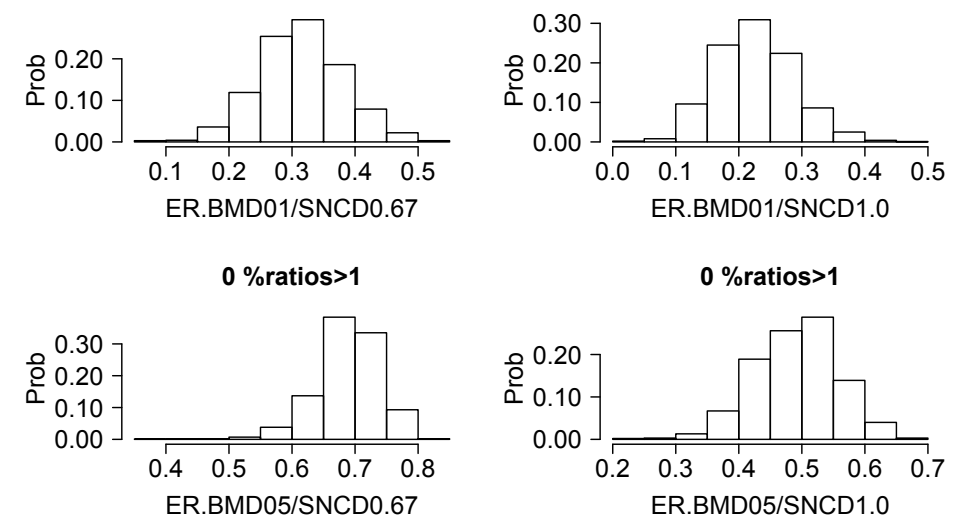

$33.9 \%$ ratios $>1$


Figure C.11: Histogram for BMD/SNCD when $\kappa=7, \eta=2, k=6, n_{i}=50$ 

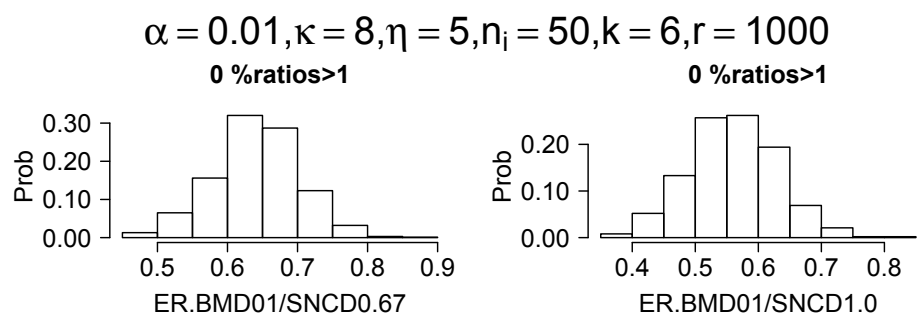

$0 \%$ ratios $>1$

$0 \%$ ratios $>1$
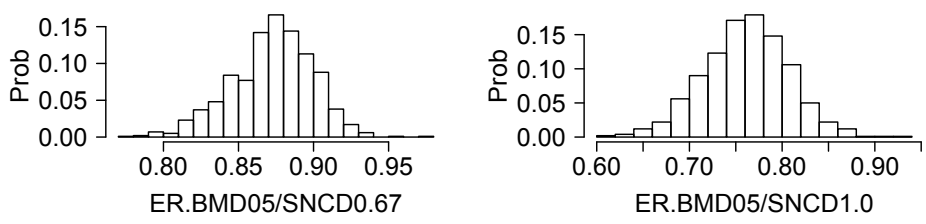

$59.4 \%$ ratios $>1$
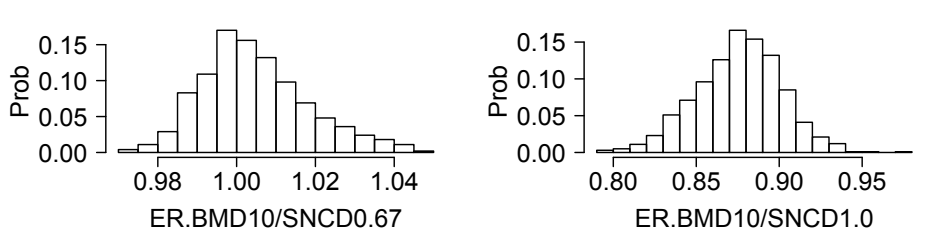

Figure C.12: Histogram for BMD/SNCD when $\kappa=8, \eta=5, k=6, n_{i}=50$

$$
\begin{array}{r}
\alpha=\underset{0 \% \text { ratios }>1}{0.01, \kappa=4, \eta}=3, n_{i}=200, k=\underset{0 \% \text { ratios }>1}{6, r}=1000 \\
0
\end{array}
$$
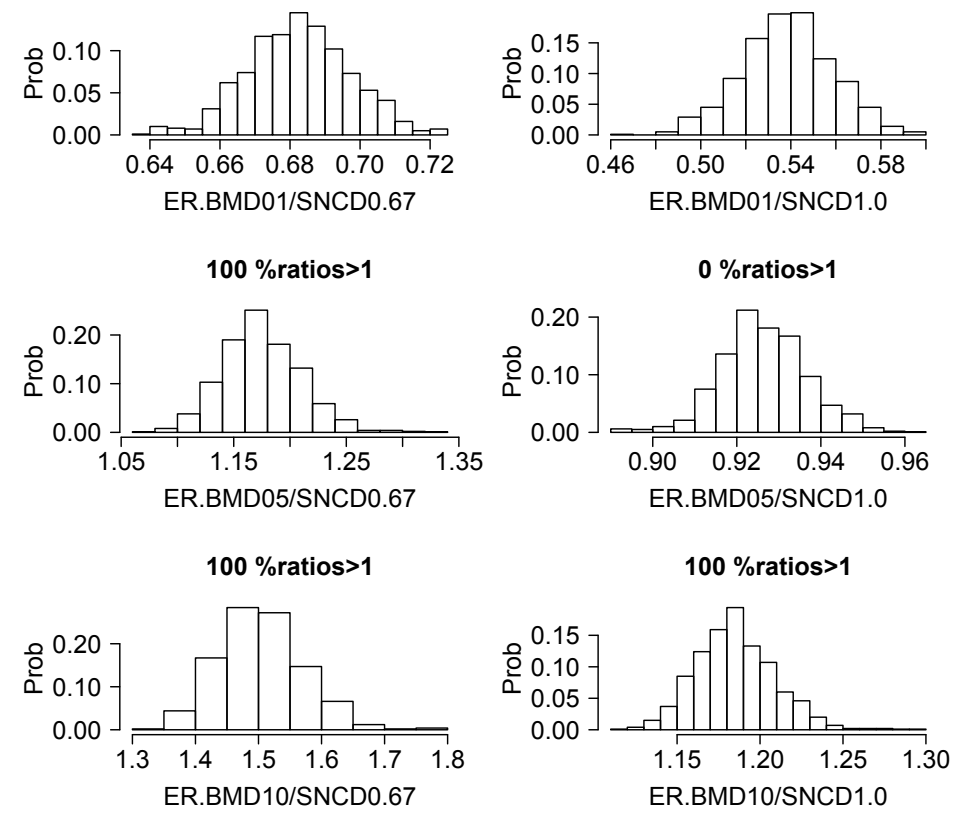

Figure C.13: Histogram for BMD/SNCD when $\kappa=4, \eta=3, k=6, n_{i}=200$ 



$96.8 \%$ ratios $>1$
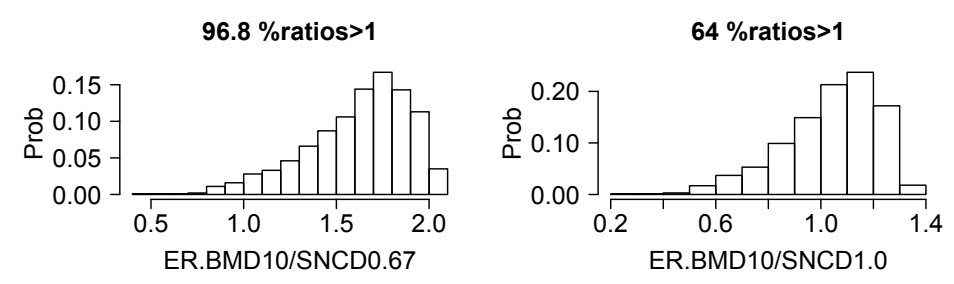

Figure C.14: Histogram for BMD/SNCD when $\kappa=5, \eta=1, k=6, n_{i}=200$

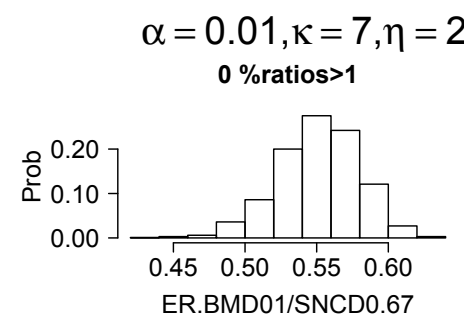

$$
\begin{array}{r}
n_{i}=200, k=6, r=1000 \\
0 \% \text { ratios }>1
\end{array}
$$


Figure C.15: Histogram for BMD/SNCD when $\kappa=7, \eta=2, k=6, n_{i}=200$ 



Figure C.16: Histogram for BMD/SNCD when $\kappa=8, \eta=5, k=6, n_{i}=200$

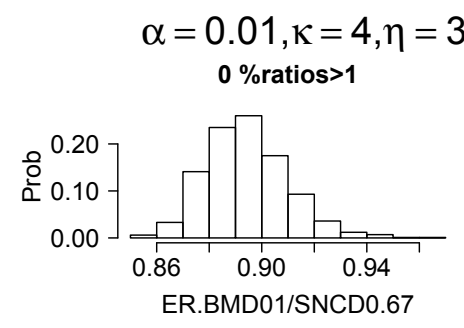

$$
\mathrm{n}_{\mathrm{i}}=500, \mathrm{k}=\underset{0 \% \text { ratios }>1}{6, r=1000}
$$
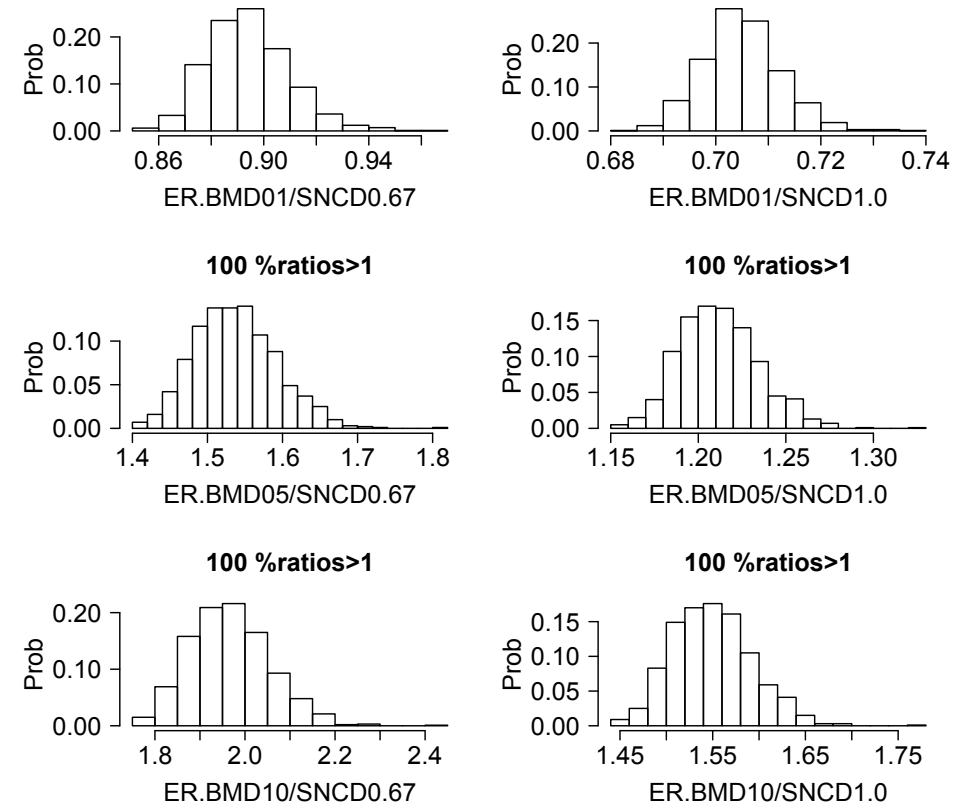

Figure C.17: Histogram for BMD/SNCD when $\kappa=4, \eta=3, k=6, n_{i}=500$ 

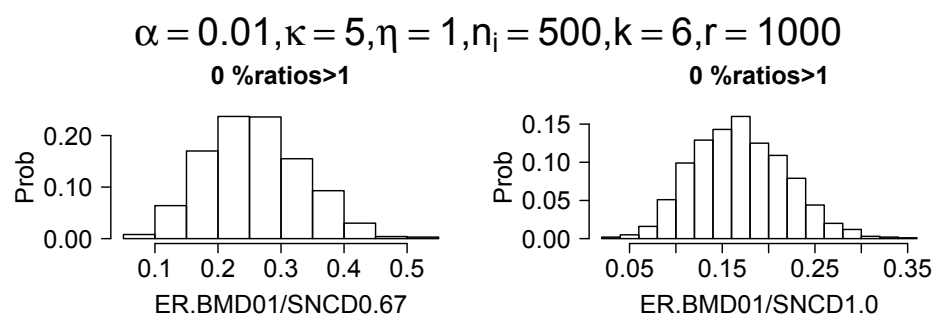

$90.5 \%$ ratios $>1$
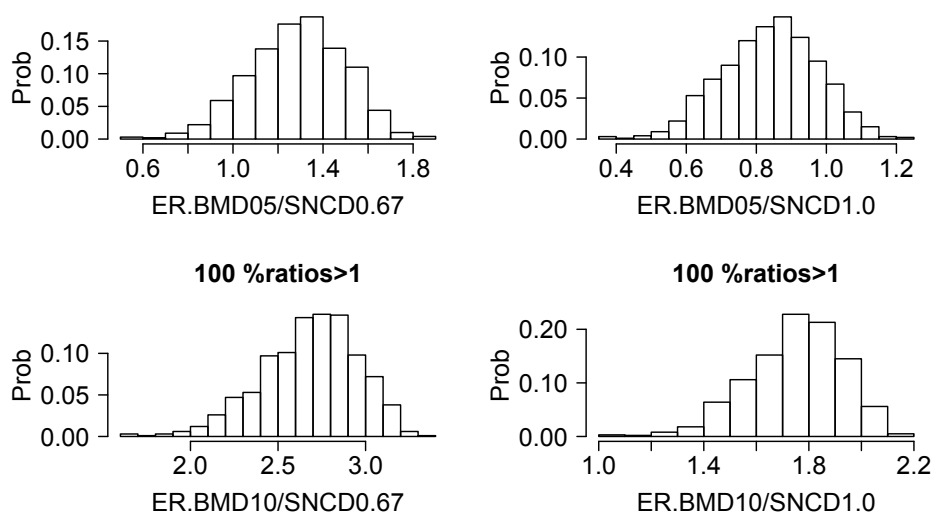

Figure C.18: Histogram for BMD/SNCD when $\kappa=5, \eta=1, k=6, n_{i}=500$



$n_{i}=500, k=6, r=1000$
$0 \%$ ratios $>1$
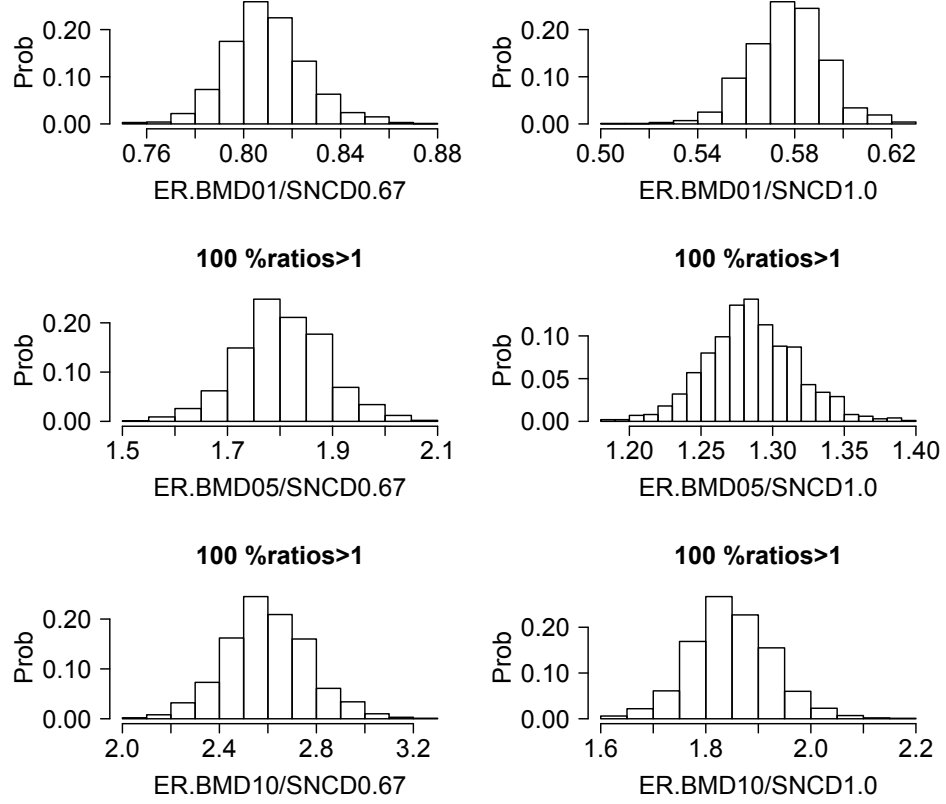

Figure C.19: Histogram for BMD/SNCD when $\kappa=7, \eta=2, k=6, n_{i}=500$ 




$\mathrm{i}=500, \mathrm{k}=\underset{\mathrm{0}}{6} \mathrm{r}=1000$
ratios $>1$
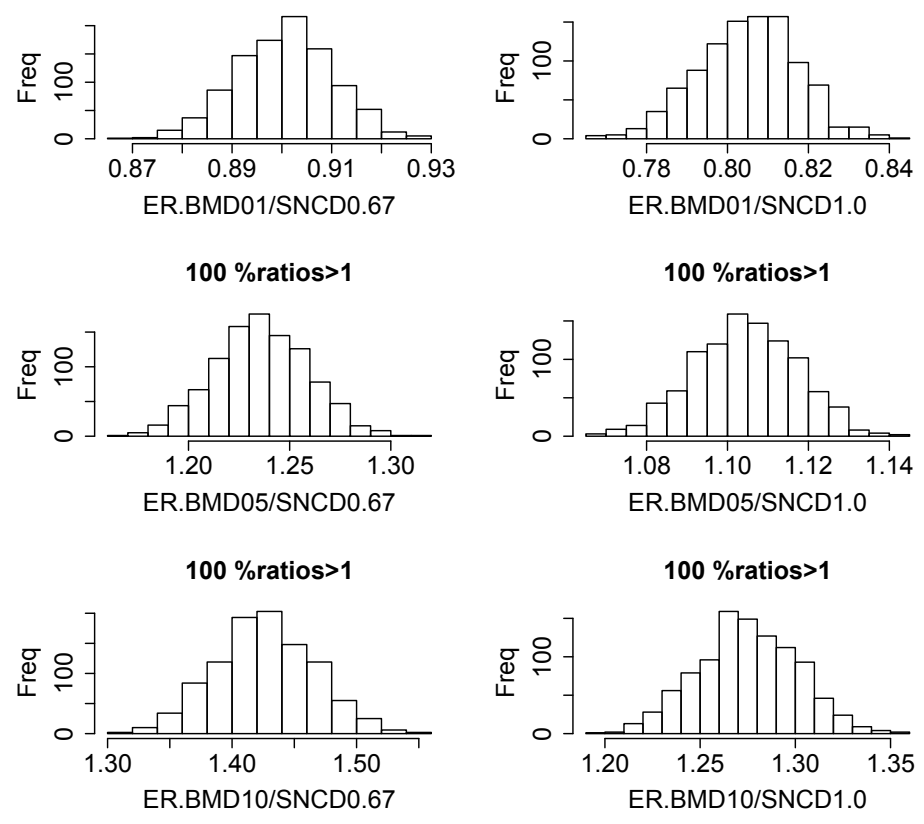

Figure C.20: Histogram for BMD/SNCD when $\kappa=8, \eta=5, k=6, n_{i}=500$ 